\title{
Pharmacological studies on the contribution of the neuropeptide proctolin to the cephalic control of singing behavior in grasshopper Chorthippus biguttulus (L. 1758)
}

\author{
Dissertation \\ Zur Erlangung des Doktorgrades \\ Der Mathematisch-Naturwissenaschaftlichen Fakultäten \\ Der Georg-August-Universität zu Göttingen
}

\author{
Vorgelegt von \\ Stoyan Raykov Vezenkov \\ aus Blagoevgrad/Bulgarien
}

Göttingen 2004 
D7

Referent: Prof. Dr. Ralf Heinrich

Korreferent: Prof. Dr. Norbert Elsner

Tag der mündlichen Prüfung: 2.11.2004 


\section{Contents}

\section{Contents}

Abbreviations
1
Introduction.

$2 \quad$ Materials and Methods.............................................................................. 15

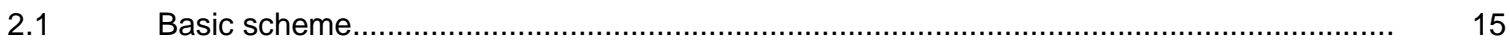

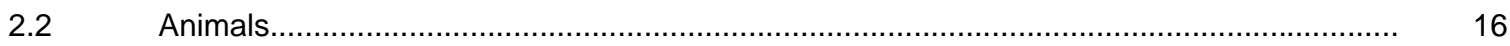

2.3 Preparation for pharmacological stimulation.......................................................... 16

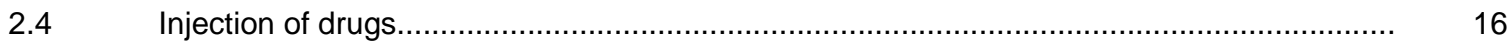

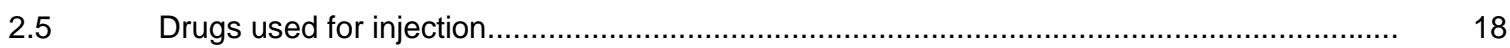

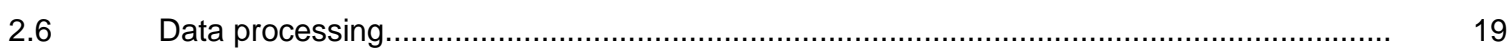

2.7 Evaluation and statistical analysis of experimental results......................................... 20

3 Results 22

3.1 Introduction to the natural acoustic communication of Ch.b........................................ 22

3.1.1 Short introduction to the physiology of the sound production in the subfamily

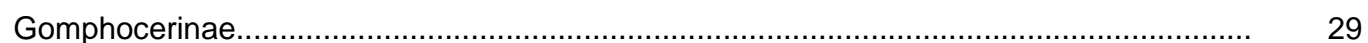

3.1.2 Pharmacological stimulated stridulation in Ch.b...................................................... 32

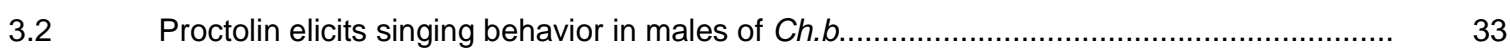

3.2.1 Other proctolin-stimulated elements of sexual behavior (courtship)............................... 37

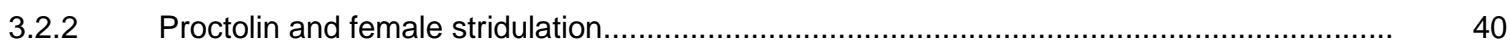

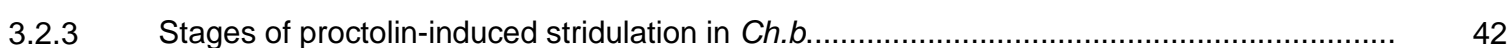

3.2.4 Dose effects of proctolin on the stimulation of stridulation.................................................. 43

3.2.5 Temporal aspects of the application of proctolin.........................................................

3.2.5.1 Optimization of the fixed intervals in between proctolin pulses........................................... 47

3.2.5.2 Short inter-stimulus intervals decrease the stimulatory effect of proctolin on stridulation of Ch.b.

3.3 Methodological problems and adjusting the stimulation protocol to the proctolin mode of

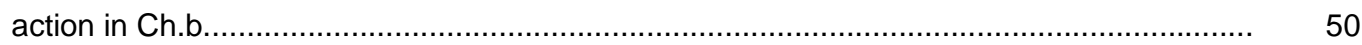

3.3.1 Repeated stimulation with excitatory vs. inhibitory drugs (Protocol 3-1-4).......................... 50

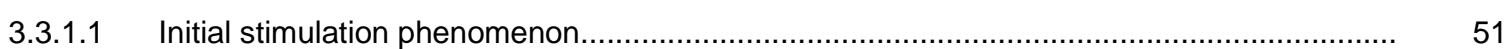

3.3.1.2 Contamination of drugs in two-barrels microcapillary.................................................. 51

3.3.2 Alternative protocol, optimized for studies with proctolin (Protocol 3-3-3).......................... 53

3.4 Proctolin- and cholinergic brain neurons control the sound production in Ch.b.................... 56 


\section{Contents}

3.4.1 Proctolin- and muscarine-sensitive sites for the stimulation of stridulation in the brain of

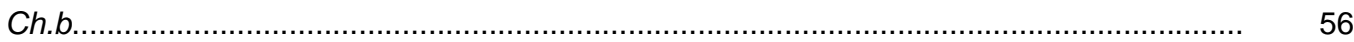

3.4.2 Comparison of proctolin- and muscarine-stimulated stridulation.................................... 58

3.4.3 Combination of proctolin- and muscarine-induced stridulation......................................... 59

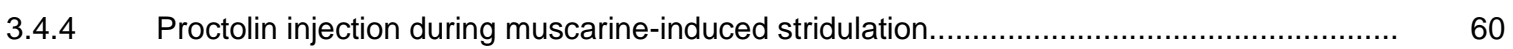

3.4.5 Necessity of mAChRs for proctolin-induced stridulation............................................... 63

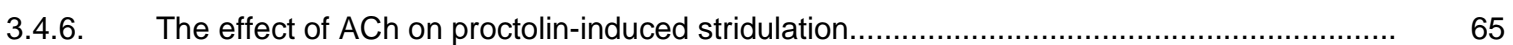

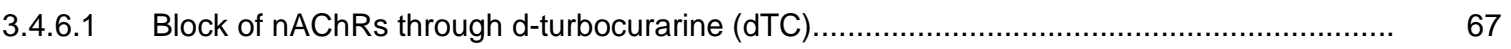

3.4.6.2 The role of AChE activity on proctolin-stimulated stridulation........................................ 69

3.5 Intracellular pathways activated by proctolin receptors in the brain of Ch.b........................ 78

3.5.1 The cAMP second messenger pathway................................................................... 78

3.5.1.1 Inhibition of AC through SQ22536 partly suppressed proctolin-stimulated stridula-

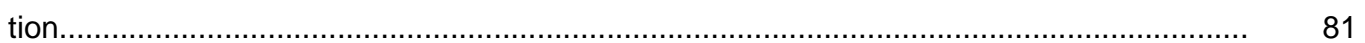

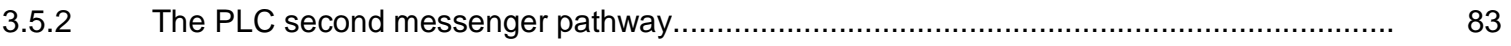

3.5.2.1 Blockade of PLC signal pathway through neomycin.................................................... 85

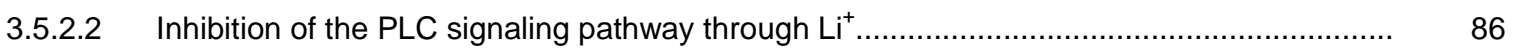

3.5.2.3 Contribution of $\mathrm{Ca}^{2+}$ signaling to proctolin-mediated excitation....................................... 88

3.5.2.4 Contribution of DAG to proctolin-stimulated stridulation................................................... 92

3.5.2.5 Potassium channels as potential effectors of proctolin receptor activation........................ 99

3.6 Effects of additional transmitter systems on proctolin-stimulated stridulation in male

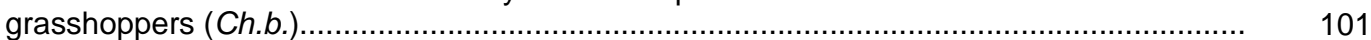

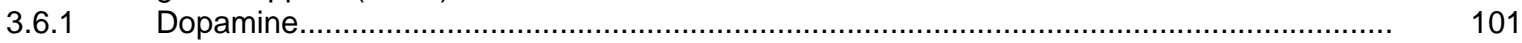

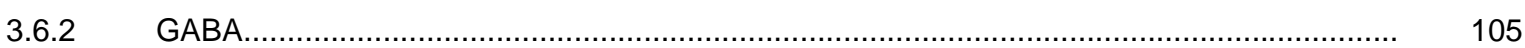

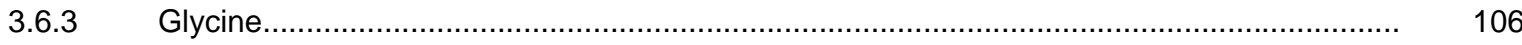

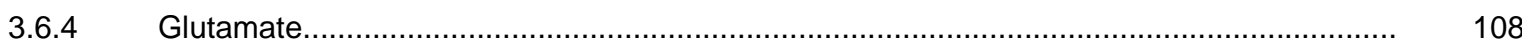

3.6.5 Histamine

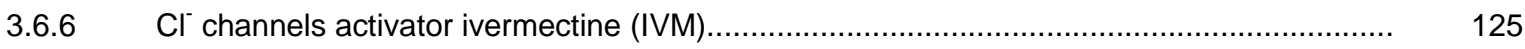

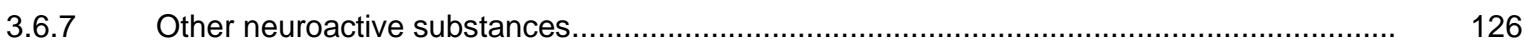

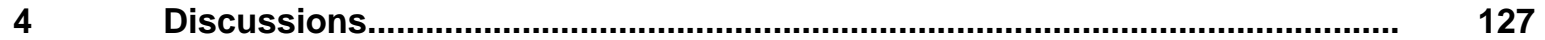

4.1 Proctolin-elicited singing behavior in Ch.b. - comparison to the natural one........................ 127

4.2 Song types and other courtship elements stimulated by proctolin...................................... 128

T.3 To dose-effects of proctolin mode of action.............................................................. 130

4.4 Temporal aspects of proctolin-stimulated stridulation................................................... 131

4.5 Possible involvement of receptor desensitization in the inactivation of proctolin-induced singing behavior (Ch.b.).

4.6 The role of cholinergig transmission on the time course of proctolin-stimulated stridula-

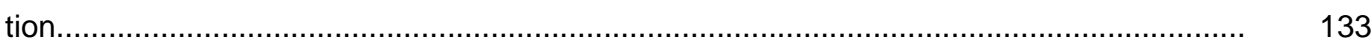

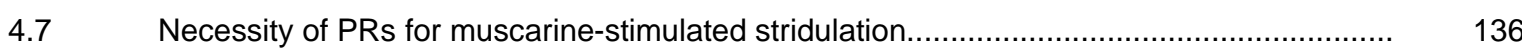

4.8 Methodological problems and optimization the stimulation protocol to the proctolin mode of action - critical discussions.............................................................................. 136

4.9 Additional remarks on the pharmacological studies.................................................... 140

4.10 Short introduction to the physiology of central nervous neuronal networks......................... 142

4.11 Second messenger signaling coupled to proctolin-stimulated singing.............................. 149

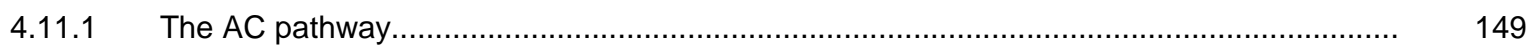

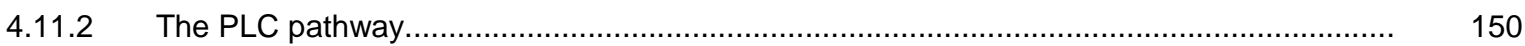

4.11.3 Conclusions about the organization of the second messenger pathways, involved in proctolin-stimulated stridulation in Ch.b. 


\section{Contents}

4.12 Modulation of singing behavior through co-transmission in the brain of Ch.b. (the role of proctolin)...

4.12.1 Singing patterns I and II of the normal song (calling and courtship).....

4.12.2 Uncoupling of the basal excitation to sing from the stridulation itself.

4.12.3 Conclusions on the role of proctolin in the courtship of Ch b

4.13 Leg stridulation and wing flapping in Ch.b. and Stenobothrus rubicundus......................... 157

4.13.1 Wing stridulation in Stenobothrus rubicundus............................................................... 158

4.14 The brain may exist components of the stridulatory pattern-generating network................ 158

4.15 Differences between the cephalic stridulatory control systems of O.v. and Ch.b................ 161

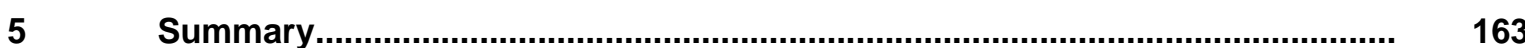

$6 \quad$ Bibliography.......................................................................................... 166

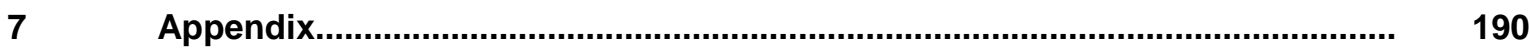




\section{Abbreviations}

$\begin{array}{ll}\text { AC } & \text { Adenylyl cyclase } \\ \text { ACh } & \text { Acetyl choline } \\ \text { AChR } & \text { Acetyl choline receptor } \\ \text { nAChR } & \text { Nicotinic acetyl choline receptor } \\ \text { mAChR } & \text { Muscarinic acetyl choline receptor } \\ \text { AN } & \text { Ascending neuron } \\ \text { AP } & \text { Action potential } \\ { }^{\circ} \text { } & \text { Degrees centigrade } \\ \text { cAMP } & \text { Cyclic adenosine monophosphate, second messenger } \\ \text { Ch.b. } & \text { Chorthippus biguttulus (L. 1758) } \\ \text { cm } & \text { Centimeter } \\ \text { CNS } & \text { Central nervous system } \\ \text { C Duration (\%) } & \text { Duration from the beginning of the first sequence to the end of the last sequence } \\ \mathrm{C}_{\mathrm{M}} & \text { Molar concentration 1M=[mol/l] } \\ \text { DAG } & \text { Diacylglycerol } \\ \mathrm{dB} & \text { Decibel } \\ \mathrm{D}_{1,2,3,4,5} & \text { Dopamine receptors of type 1, 2, 3, 4, 5 } \\ \text { DN } & \text { Descending neuron } \\ \text { DUM } & \text { Dorsal-unpaired-median neuron } \\ \text { EPSP } & \text { Excitatory postsynaptic potential }\end{array}$

Fig. $\quad$ Figure

g Gram

$\mathrm{G} \quad$ Ion conductance through the membrane $\left(\mathrm{G}_{\mathrm{K}}-\mathrm{K}^{+}\right.$conductance)

GABA $\quad \gamma$-Aminobutyric acid

$\mathrm{GABA}_{\mathrm{A}} \quad$ Ionotropic GABA receptors (GABA-gated $\mathrm{Cl}^{-}$channels)

$\mathrm{GABA}_{B} \quad$ Metabotropic GABA receptors (G-protein coupled receptors)

GPCR G-protein coupled receptor, triggering second messenger cascades

iGluRs Ionotropic glutamate receptors - AMPA-Rs, Kainate-Rs and NMDA-Rs

$\mathrm{IP}_{3} \quad$ Inositol 1,4,5-triphosphate

IPSP Inhibitory postsynaptic potential

HLs Hind legs

$\mathrm{Hz} \quad$ Hertz

I Liter

mAChRs Muscarinic ACh receptors 


\section{Abbreviations}

$\begin{array}{ll}\mathrm{M}_{1,2,3,4,5} & \text { Muscarinic ACh receptors of type 1, 2, 3, 4, 5 } \\ \mathrm{mGluRs} & \text { Metaobotropic glutamate receptors } \\ \mathrm{ml} & \text { Milliliter } \\ \mathrm{mm} & \text { Millimeter } \\ \mathrm{mM} & \text { Milimolar concentration } \\ \mathrm{msec} & \text { Millisecond } \\ \mathrm{mV} & \text { Millivolt } \\ \mu \mathrm{m} & \text { Micrometer } \\ \mathrm{n} & \text { Number of experimental animals } \\ \mathrm{nA} & \text { Nanoamper } \\ \mathrm{nm} & \text { Nanometer } \\ \text { O.v. } & \text { Omocestus viridulus (L. 1758) } \\ \text { PD } & \text { Pulse duration (in sec) } \\ \text { PhE } & \text { Phorbol-12,13-dibutirate (phorbol ester) } \\ \mathrm{PNS} & \text { Peripheral nervous system } \\ \text { PTX } & \text { Picrotoxin } \\ \text { PLC } & \text { Phospholipase C } \\ \text { PLI } & \text { Proctolin-like immunoreactivity } \\ \text { PRs } & \text { Proctolin receptors (G-protein coupled receptors) } \\ \text { PKA, PKC } & \text { Proteinkinase A, C } \\ \text { SD } & \text { Standard deviation } \\ \text { sec, msec } & \text { Second, milisecond } \\ \text { S Duration (\%) } & \text { Total sequence duration after normalization in (\%) } \\ \text { V } & \text { Volt } \\ \text { vs. } & \text { Versus } \\ \bar{X} & \text { Average value } \\ & \end{array}$




\section{Introduction}

\subsection{Proctolin}

Proctolin (RYLPT) was the first neuropeptide to be isolated and sequenced from insects (Brown, 1975) and was subsequently found to have wide distribution throughout the arthropods (Osborne, 1996; Konopinska, 1999). First proctolin has been reported (Brown, 1967) as a "gut factor" which caused slow graded contractions of proctodeum longitudinal muscles in the cockroach Periplaneta americana. The "factor", extracted from the fore- and hindguts of Periplaneta americana, mimicked closely the neurally evoked responses in that tissue.

The structure of proctolin was determined (Starrat and Brown, 1975) as $\mathrm{H}_{2} \mathrm{~N}$ Arg-Tyr-Leu-Pro-Thr-COOH. Proctolin was synthesized, and the structure confirmed by showing that the synthesized molecule had the same physical, chemical and biological properties as the active molecule in the purified extracts.

It has been reported (Orchard et al., 1989) that proctolin is present in a number of tissues in the majority of insects tested. The functions of proctolin are still unclear in many cases. Proctolin seems to have roles in the CNS, the control of movement of the gut, heart function and in reproduction. Thus it might be considered a vital neuromodulator and neurohormone in many insects. 


\subsection{Distribution of proctolin in the CNS of insects}

The distribution of proctolin throughout the insects has been shown in different studies (O'Shea, 1981; 1982). A short introduction into immunoreactivity to
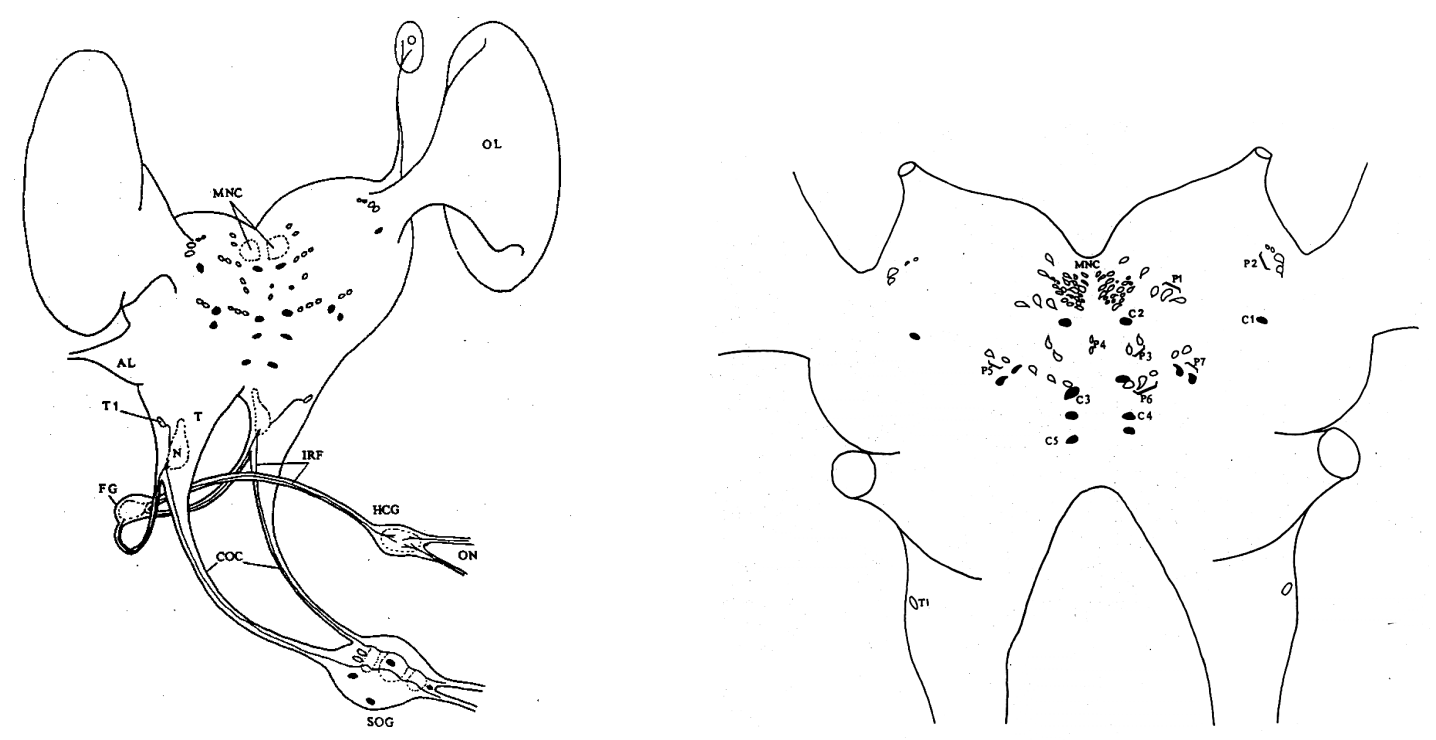

Fig. 1-1 Proctolin-like immunoreactivity (PLI) in the brain of Schistocerca gregaria. PLI can be seen in nerve fibers in the mushroom bodies, weakly in the pars inercerebralis and strongly in the cell bodies located on the lateral margins of the protocerebral lobes. One set of nerve fibers appear to ramify in the central complex (from Issberner, 2001)

proctolin in CNS of Schistocerca gregaria will be presented here (Issberner, 2001). The distribution of the neuropeptides throughout the insects differs significantly and should be studied extensively within each particular system, examined. Any generalization or transfer of data from another insect species should be excluded.

Proctolin-like immunoreactivity has been revealed in the tritocerebrum, and the protocerebrum, with a total of approximately 50 cell bodies labeled in each half of the brain (Fig. 1-1; from Issberner, 2001). Approximately 40 weakly immunoreactive somata have been consistently observed, located in the pars intercerebralis (Issberner, 2001). This is the location of the median neurosecretory cells found in the dorso-anterior section of the brain (Williams, 1975). On either side of these immunoreactive cell bodies a group of three immunoreactive somata has been determined. Only one group of four immunoreactive cell bodies has been observed in the cortex of the antennal lobe - the larger of these cells has appeared to project axons towards the calyx where a newtwork of immunoreactive fibers exists. One fiber from this neuropil has projected onto the lateral margins of the 
central complex where it has appeared to ramify (Issberner, 2001). The remaining immunoreactive somata have been observed in bi-laterally symmetrical groups of two and three cells whose axons have not been traced. No singly located immunoreactive somata have been detected in the anterior portion of the brain, however the posterior protocerebrum has contained ten singly located immunoreactive somata, with five on each side of the midline (Issberner, 2001).

\section{PLI has located a series}

of groups immunoreactive somata in suboesophagal ganglion (Issberner, 2001). Immunoreactive fibers have been observed in the circumoesophagal connectives. These have appeared to traverse the length of the ganglion running on either side of the midline in the dorsal section of the ganglion and projects into the posterior connectives (Issberner, 2001).

Approximately 8 immunoreactive somata have

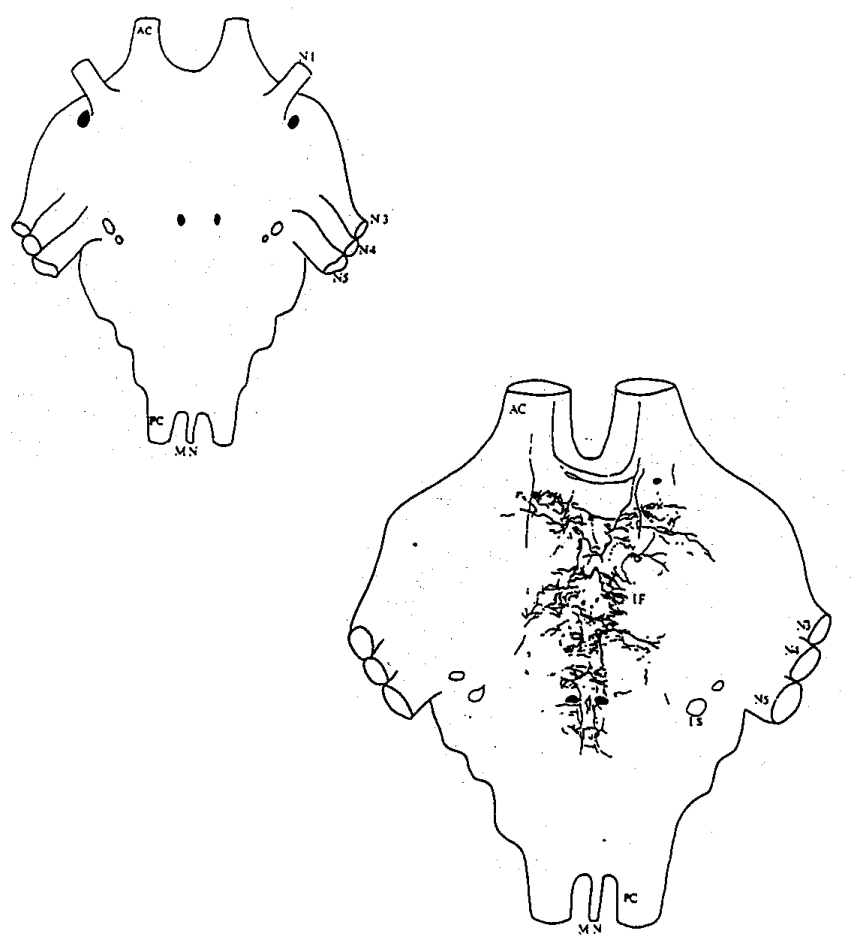

Fig. 1-2 PLI in methathoracic ganglion of Schistocerca gregaria. Plan of the immunoreactive somata (up); and map of the immunoreactive fibers (down) reconstracted from tracings prepared with antisera to proctolin (from Issberner, 2001)

been revealed by the preparation of serial sections of the methathoracic ganglion with antiserum to proctolin (Fig. 1-2; from Issberner, 2001). The most anterior two are bi-lateral pair in the ventral plane of the ganglion. Two other ventral somata have been located as a pair in the central medial part of the ganglion, and the remaining four immunoreactive somata, are arranged as two pairs of bi-laterally symmetrical cells approximately adjacent to the root of nerve five in the dorsolateral rind (Fig. 1-2 up; Issberner, 2001). Immunoreactive fibers have also been identified in the methathoracic ganglion, and could be characterized as a course network of fibers. In general the fibers have run longitudinally through the ganglion with areas along its length seemingly to have an increased number of transverse 
immunoreactive fibers (Issberner, 2001). Immunoreactive fibers have also been detected in the anterior connectives running into the complex arrangement of fibers within the ganglion (Fig. 1-3 down; Issberner, 2001).

A sexual dimorphism has been shown for the distribution of proctolin in both the numbers, and distribution of somata containing immunoreactive material in the terminal ganglion of Schistocerca gregaria. This may indicate sexual related functions of the proctolin-like immunoreactive material in Schistocerca gregaria.
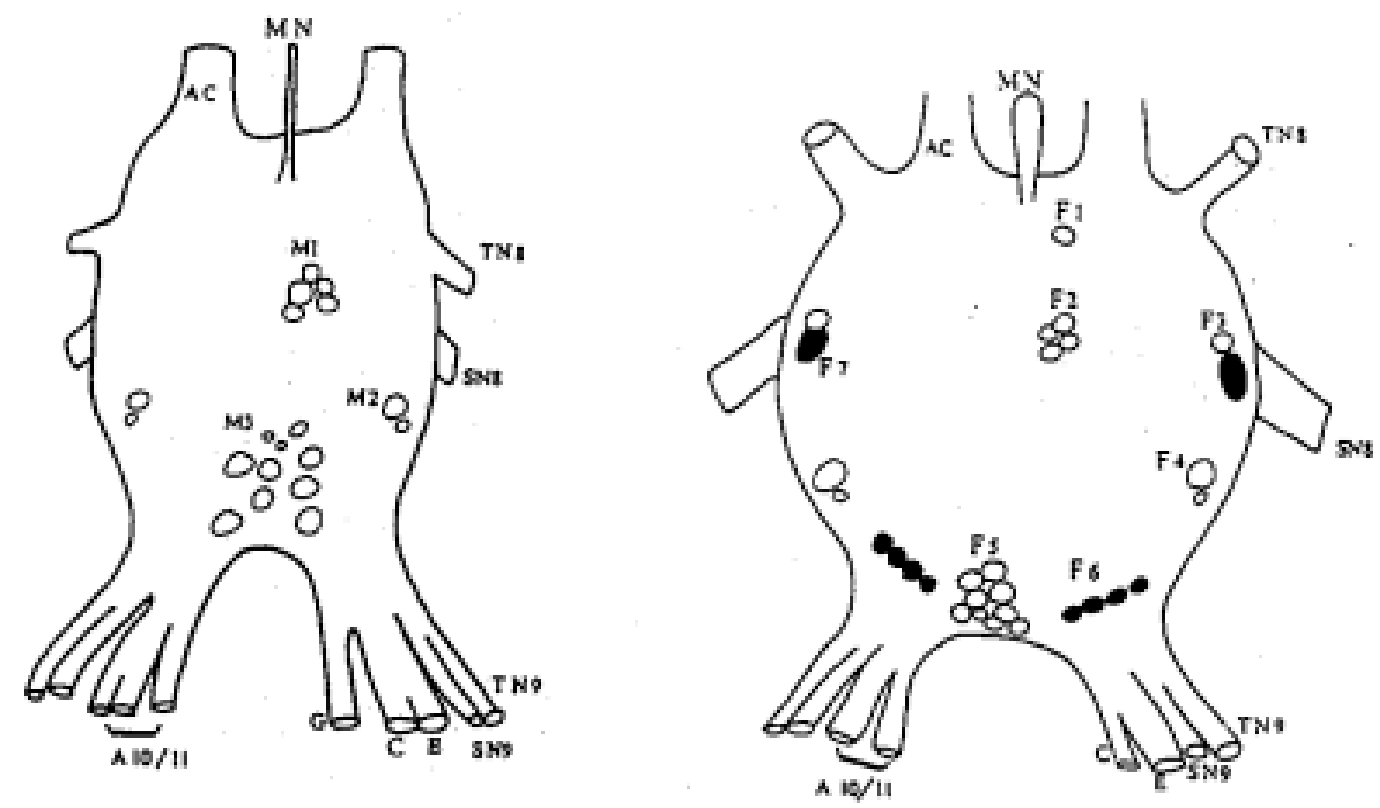

Fig. 1-3 PLI in the terminal ganglion of Schistocerca gregaria

Plan of the immunoreactive somata observed by reconstruction of serial section of the male terminal ganglion (left); and corresponding plan of immunoreactive somata in the terminal ganglion of female Schistocerca gregaria (right) (Issberner, 2001).

This overview shows that proctolin is widely distributed throughout the CNS of Schistocerca.

\subsection{Neuropeptide release}

Neuropeptides are stored in vesicles at their release sites in axon terminals or in en passant varicosities until a depolarization induces their release by exocytosis (Zupanc, 1996). Release can occur either at typical synapses or at nonsynaptic sites ("target" structures). Thus peptides may act at a short distance in a 
strictly localized fashion (common for parts of CNS) or at some distance as a local or systemic neurohormone with a more diffuse set of targets (Nässel, 2002).

\subsection{Neuropeptide receptors}

The majority of the neuropeptides studied act on Gprotein coupled receptors (Zupanc, 1996; Strand, 1999; Hewes and Taghert, 2001; Nässel, 2002) (right). These receptors are known to interact with either adenylate cyclase (AC) or the phospholipase $\mathrm{C}$

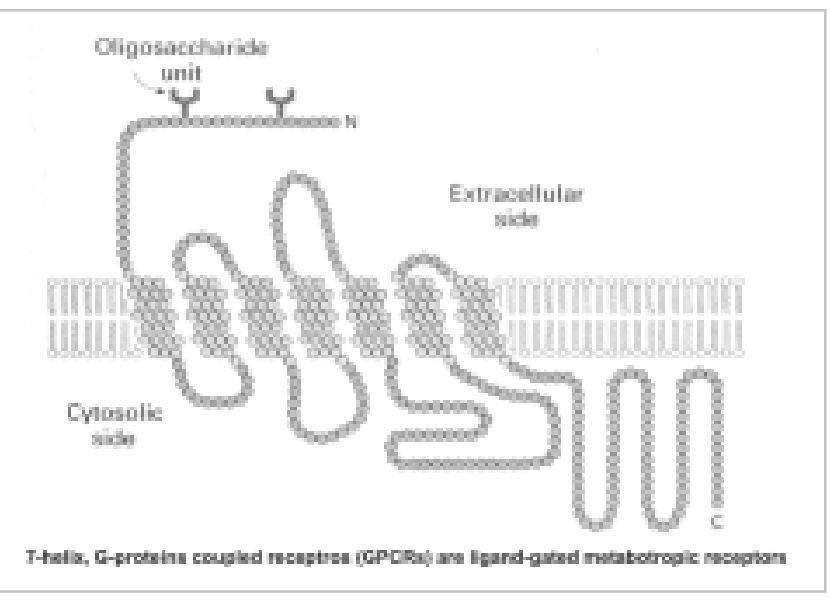
pathway (phospholipid hydrolysis) and thus regulate the production of second messengers such as cAMP, diacylglycerol (DAG) and inositoltriphosphate $\left(\mathrm{IP}_{3}\right)$ and also regulation of intracellular levels of $\mathrm{Ca}^{2+}$. Some receptors-coupled $\mathrm{G}$ proteins also act without involvement of second messengers (Quartara and Maggi, 1997; Hammond, 2001) by directly interacting with ion channels. The cellular responses to activation of GPCRs are, for instance, regulation of ion channel activity, activation (or inactivation) of various protein kinases and/or protein phosphatases (e.g. leading to receptor inactivation or interference with transmitter release), activation of transcription factors and protein synthesis. Thus GPCRs are commonly responsible for pre- and postsynaptic modulatory actions of short and long term duration. 


\subsection{Proctolin Receptors}

To date only one proctolin receptor in Drosophila has been identified and characterized. Previously proctolin has been shown to signal through heterotrimeric G proteins in the cockroach (Mazzocco-Manneval et al., 1998). Proctolin receptors are 7-helix G-proteins coupled receptors (GPCRs), which are ligandactivated metabotropic receptors.

Proctolin has dual effects on insect neuromuscular junctions - it potentiates the amplitude of neurally evoked (L-glutamate mediated) contractions and also directly evokes contractions (Lange and Orchard, 1984). The range of proctolin effects in insect muscle, including the elevation of inositol-trisphosphate $\left(\mathrm{IP}_{3}\right)$, potentiation of calcium influx, and reduction of resting potassium conductance, is indicative of multiple receptor subtypes (Baines et al., 1990; Lange et al., 1987; Walther and Zittlau, 1988). It has been revealed by using different proctolin analogues with differential effects that its action is mediated by at least two receptor subtypes on the extensor tibiae muscle of Schistocerca gregaria (Baines et al., 1996).

Firstly a membrane protein that specifically binds the insect neuropeptide proctolin was purified using standard chromatography from cockroach foregut membranes (Mazzocco and Puiroux, 2000).

Than a G-protein coupled receptor (encoded by CG6986) for proctolin in Drosophila melanogaster has been identified and characterized (Johnson et al., 2003). Proctolin receptor immunosignals have been found in the hindgut, heart and in distinct neuronal populations of the CNS.

Information regarding locations and times of proctolin receptor expression can be of value in the interpretation of proctolin signaling. For example, several sites on which proctolin was assumed to act directly, such as hindgut and heart, have been immunolabeled with antibodies against proctolin receptors (Johnson et al., 2003). Several groups have postulated a role for proctolin in regulating the heart rate of various insects. It has not been observed staining in the heart proper, but did observe minor staining in the anterior aorta and in some nerve terminals apposed to cardiac tissue. There are conflicting reports about proctolin acting on the heart rate in Drosophila. Given the mechanism of cardiac pacemaking and its 
modulation (Johnson et al., 2002), any direct action of proctolin on heart muscle is likely to be excitatory, whereas indirect action onto neurons that innervate the heart could have a net excitatory or inhibitory action (McGaw et al., 1995). Proctolin receptor immunosignals on neurosecretory cells suggest that the peptide may act as a releasing (or inhibiting) factor of other, specific peptide hormones (Johnson et al., 2003). Identifying those cells, and relating their secretory products to proctolin actions, may be a useful step in providing detail to neural circuits that control discrete physiological functions. An unexpected role for proctolin in visual processing was indicated by the presence of both peptide and receptor immunostaining in the medulla and lamina. Likewise, the expression of receptor immunosignals in pericardial cells suggests that proctolin may have unpredicted roles in cardiac physiology (Johnson et al., 2003).

A close correlation between the distribution of proctolin and proctolin receptors has been shown (Fig. 1-4, Johnson et al., 2003). The discovery of a candidate proctolin receptor will promote further studies of proctolin-signaling mechanisms underlying synaptic modulation. However, the precise details of the regulatory effects depend on which proctolin neuron is activated, and the mechanisms that pattern such fundamental synaptic modulation are unknown for most functional circuits. Clearly, information about sites of proctolin receptor expression will present a useful means with which to pursue such issues (Johnson et al., 2003). The ability to assay for proctolin receptor expression will strengthen efforts to interpret the occurrence and significance of proctolin modulation in arthropods (Johnson et al., 2003).
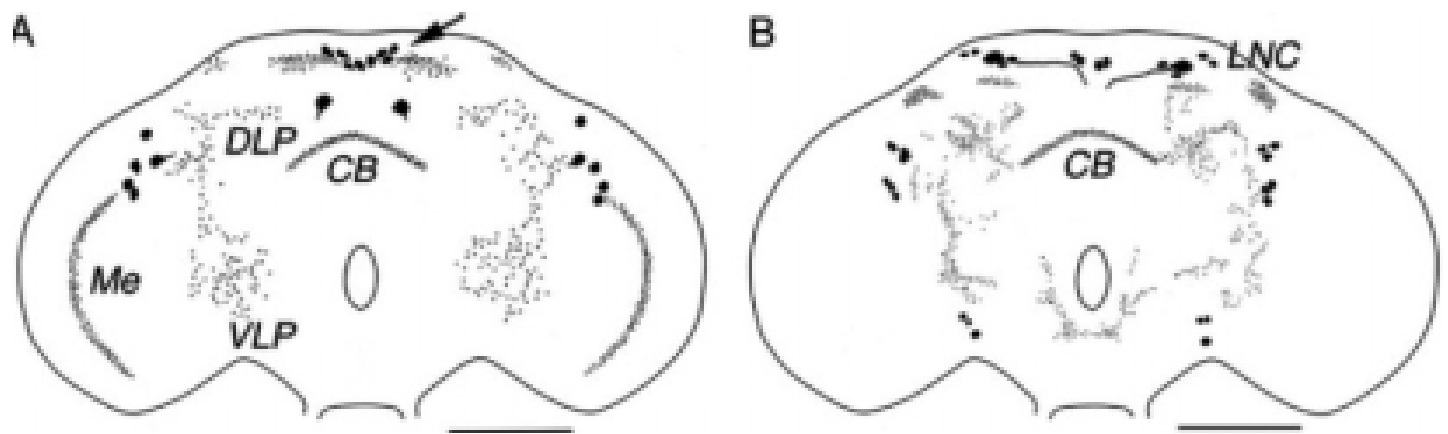

Fig. 1-4 Immunolabeling in Drosophila brain with antiserum to proctolin receptor $\boldsymbol{A}$ and to proctolin peptide (B). (from Johnson et al., 2003) 


\subsection{Neuromodulatory and hormonal actions of neuropeptides}

Insect neuropeptides can act as modulators or neurohormones, but there is yet no evidence for clear neurotransmitter function. Only one identified FMRFamide-gated channel in molluscs (Helix) has been identified so far (Lingueglia et al., 1995; Cottrell, 1997) but no peptide-gated channels have been identified in insects (Nässel, 2002). A neurohormone, in the strict sense, is released into the circulatory system and acts after transport over considerable distances. Less strictly one talks about local neurohormones that are released in a paracrine fashion by the neurons in the central nervous system and diffuse within the tissue over some distance before reaching specific receptors. In the case of local neurohormones, no specific neurohemal organs or release areas need to develop.

The terms neurotransmitter and neuromodulator also deserve further comment. A neurotransmitter is released at a synapse and produces a rapid and transient response by acting directly on ligand-gated ion channels (primary signal for opening or closing of ion channels). A neuromodulator, released synaptically or non-synaptically, can produce a host of effects (other than acting directly on gating of ion channels) commonly by acting on G-protein-coupled receptors (GPCRs). A strict definition (Lopez and Brown, 1992) is that neuromodulator is such regulation of ion channel gating by extrinsic signals that use mechanisms that are not the primary way by which these channels are opened or closed (voltage or ligands). Channel modulation entails direct modifications of ion channel activity through phosphorylation, dephosphorylation, direct interactions with Gproteins or protease action. The modulatory action can be presynaptic or postsynaptic and serve to prolong or shorten the effects of a co-released classical transmitter or to strengthen or diminish it. Neuromodulation can be result from both by peptide release within the CNS and by circulating peptides. Neuropeptides can act as neuromodulators in two principal ways:

1. either they act locally as intrinsic modulators that are released by one or more circuit neurons to affect synapses or membranes within that circuit; or

2. they act more globally, as extrinsic modulators, affecting multiple circuits and even peripheral targets (Katz and Frost, 1996; Katz, 1998) 
The role of peptides in neuromodulation has been extensively studied in the periphery, for instance, at the synapse between motoneurons and muscle. Modulatory roles in central circuits are far more complex to study but have been successfully approached in a few simple rhythm-generating circuits such as the stomatogastric ganglion of crustaceans (Marder et al., 1997; Nusbaum et al., 2001).

An early demonstration of the modulatory role of a peptide in insect was that of proctolin potentiating the action of the excitatory transmitter glutamate at certain neuromuscular junctions in insects (Adams and O'Shea, 1983; O'Shea and Schaffer, 1985; O'Shea et al., 1985). Release of glutamate probably occurs at lower frequencies of stimulation and proctolin is additionally released at higher frequencies leading to the combined effect of increased muscle tonus over longer periods of time.

Most insect neuropeptides are likely to be multifunctional, and many of them can serve as synaptically released neuromodulators, as local neurohormones (neuromodulators) and as circulating neurohormones. The actions of hormonal peptides at peripheral targets commonly occur at rather low concentrations, in the nanomolar range or below. The concentrations required at central synapses are harder to estimate but may be substantially higher (Nässel, 2002).

\subsection{Neuropeptide inactivation}

Neuropeptide action is terminated by desensitization of receptors and by enzymatic degradation of the peptide (McKelvy and Blumberg, 1986; Zupanc, 1996; Strand, 1999; Nässel, 2002). These mechanisms can be relatively rapid and they probably both contribute to the termination of a peptides' action on the receptor. Desensitization is caused by receptor phosphorylation and/or by receptor down regulation (Garland et al., 1996; McConalogue et al., 1998). Neither of these desensitization mechanisms has been studied in insects. A number of peptidases responsible for neuropeptide inactivation have, however, been identified in insects (Turner et al., 2001; Isaac et al., 2002).

\subsection{Effects of proctolin in insects}

There can be no doubt as to the importance of proctolin as a neuroregulatory substance in insects. Where proctolin does occur (Orchard et al., 1989), it appears to function in diverse roles, far exceeding its originally suggested role as a hindgut 
neurotransmitter (Brown, 1967). Proctolin appears to be involved in the control of both visceral and skeletal muscles (normally co-expressed with glutamate) in insects, as well as possibly being involved in central processes such as a „sensitization" of the cercal escape circuit (Fitch and Djamgoz, 1988).

The antennal heart of Periplaneta americana, a small accessory circulatory pump in the head, shows a rhythmicity with myogenic automatism. The muscle fibers extending throughout of the dilator muscle are electrically coupled. Among the peptides, proctolin causes a dose-dependent strong excitation, (Hertel and Penzlin, 1992).

In Locusta migratoria stronger tetanic contractions of both scape muscles (antennal muscles) were regularly followed by post-contractions, which slowly decreases in amplitude with repeated stimulation. These findings presented proctolin as an endogenous modulator within the locust antennal motor system (Bauer, 1991).

It has been postulated that proctolin acts as a modulator of glutamateinduced contraction of the hindgut of Periplaneta americana (Cock and Holman, 1979). This conclusion was based on three suppositions: 1) A neurotransmitter should have only a transient effect on the post-synaptic structure which proctolin does not have; 2) At threshold concentrations proctolin can be seen to modulate the myogenic rhythmic contraction of the hindgut: This was not considered to be transmitter mediated; 3) Proctolin is able to enhance neurally evoked responses which was thought to be modulatory in nature. It was demonstrated also (Cook and Holman, 1985), that proctolin was able to cause contraction in a fully depolarized tissue, an observation, which was interpreted as evidence of a nontransmitter role.

As with visceral tissues, proctolin seems to have differing modes of action on different skeletal muscles. It has been proposed (Piek et al., 1978) that proctolin acts as a specific neuromodulator of the extensor tibia muscle of Locusta migratoria. Under normal circumstances there is little or no myogenic rhytmicity, but on the addition of as little as $10^{-10} \mathrm{M}$ proctolin a myogenic rhythm was established. At the neuromuscular junction of the co-axial depressor muscle in Periplaneta americana however, it has been proposed that proctolin acts as a co-transmitter with 
glutamate (Adams and O'Shea, 1983) and as a "homosynaptic modulator" or a "modulator of a co-transmitted neurotransmitter" (Adams and O'Shea, 1986).

Application of $10^{-7} \mathrm{M}$ proctolin to the neurogenic spider heart typically caused increases in the strength and rate of heart contractions and occasionally produced a sustained contracture of the myocardium (Groome et al., 1991).

The sensitivity of receptors may also be modulated at the peripheral level, before the sensory information reaches the central ganglion. Fibers, which provide sensory feedback to the central nervous system, are sensitive to proctolin, octopamine and serotonine (Pasztor, 1989). Proctolin acts by facilitating the receptor depolarization, and therefore its spiking pattern. Proctolin has always been found to exert an excitatory action in all sensory receptors studied. The change in excitability could be due to a change in ionic conductances in the sensory cells. Proctolin could influence the targets following two ways: one is through the hormonal haemolymph circulation the other could be a direct synaptic release onto the sensory receptor cells.

To function as a neurohormone, proctolin must be found in neurons, which are located such that its release into the haemolymph is possible. In the blowfly Calliphora erythrocephala, neurons containing proctolin-like immunoreactivity have been described as being located outside the neural sheath (Nässel and O'Shea, 1987). In the median neurosecretory cells and in the corpora cardiaca corpora allata complex of the Lepidopteran species Lymantria dispar (Davis et al., 1989). Measurements of proctolin in the haemolymph of Leucophaea maderae (Kingan and Titmus, 1983) have supported the role of proctolin as a neurohormone. However, some of the most convincing evidence of its hormonal role comes from studies of crustacean pericardial organs (Schwarz et al., 1984) and neurosecretory cells in the CNS (Wood et al., 1996) where it has been shown to be located and even implicated in seasonal hormonal changes (Wood et al., 1996).

A possible role of proctolin as either a neurohormone, or releasing factor of other neurohormones such as $\mathrm{AKH}$, has been proposed by the detection of proctolin-like immunoreactive material in both the storage and glandular lobes of the corpora cardiaca and in the median neurosecretory cells of the pars inercerebralis (Issberner, 2001). Later studies have demonstrated proctolin receptor immunosig- 
nals on neurosecretory cells suggesting that the peptide may act as a releasing (or inhibiting) factor of other, specific peptide hormones (Johnson et al., 2003).

\subsection{Proctolin structure activity relationships (proctolin analogues)}

Proctolin is a pentapeptide, and has the amino acid sequence $\mathrm{H}_{2} \mathrm{~N}$-Arg-Tyr-Leu-Pro-Thr-COOH. The most likely conformation of proctolin is illustrated. (right taken from Issberner, 2001).

Proctolin was first isolated,

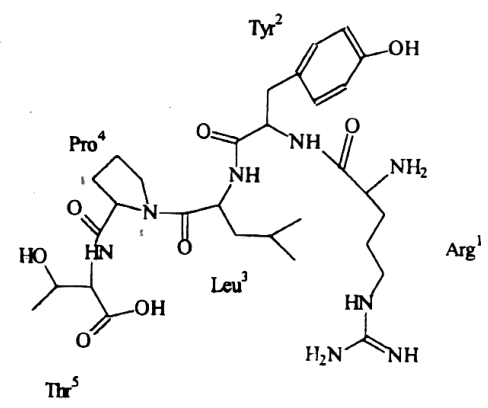
and tested for myogenicity on the proctodeal muscle of the cockroach Periplaneta americana (Brown, 1967, 1975). Since that time much work has been done to determine the structure activity relationships between the amino acid residues of proctolin and its receptor (Gray et al., 1994; Konopinska et al., 1997; Kuczer et al., 1999). In addition, the antagonistic effects of certain proctolin analogues have been characterized (Gray and Osborne, 1994; Gray et al., 2000). During this process, it became clear that the results obtained using some analogues were not the same in all insect tissues and species, and it was suggested that there were at least two sub-types of proctolin receptors in the locust (Baines et al., 1996).

\subsection{Singing behavior of grasshoppers as a suitable preparation for pharmacological investigation of its cephalic control}

The stridulatory system of gomphocerine grasshoppers has turned out to be a suitable preparation for investigating the connections between a complex behavior with its underlying neuronal activity and (extracellular) neurochemical properties. Male gomphocerine grasshoppers perform a variety of different speciesand context specific songs that are used for intraspecific communication (Jacobs, 1953, Elsner, 1974; reviewed by Elsner, 1994). The muscles responsible for the sound generating hindleg movements are activated by a thoracic patterngenerating network (Ronacher, 1989; Hedwig, 1992; Schütze and Elsner, 1995), 
which is controlled by the brain via descending command neurons (Hedwig, 1994; 1995). These neurons have dendritic arborizations in the protocerebrum dorsal and posterior to the central body complex (Hedwig, 1994). In Omocestus viridulus (O.v.) three different types of command neurons have been identified, each being responsible for one specific movement pattern (Hedwig and Heinrich, 1997). All of them elicited stridulation (O.v.) at higher spiking rate (Hedwig, 1995). Earlier pharmacological studies on this preparation revealed a central role of cholinergic activation and chloride channel-mediating inhibition in the control of stridulatory behavior (Heinrich et al., 1997; 1998).

The principal aim of the present $\mathrm{PhD}$ Thesis was to characterize the role of proctolin in the control of the singing behavior in the grasshopper Chorthippus biguttulus (Ch.b.).

For this purpose the following specific questions were addressed:

- Is stimulation of proctolin-initiated signaling pathways in the protocerebrum sufficient to induce species-specific stridulation in male and female Ch.b.?

Comparison of proctolin-induced with natural and muscarine-stimulated patterns.

- Which signalling pathway(s) mediate the stimulatory effects of proctolin? Ionotropic or metabotropic mechanism?

Second messenger pathways - adenylate cyclase and/or phospholipase C?

Ion channels?

- Direct comparison of proctolin- and muscarine-initiated stridulation. Overlap of stimulation sites?

Intensity, latency and time course of stimulated activity after single and repeated stimulation? 
- Alteration and modulation of proctolin-stimulated stridulation through costimulation of other signalling pathways.

Acetylcholine via nicotinic and muscarinic receptors

Dopamine

GABA, glycine, histamine

Glutamate 


\section{Materials and Methods}

\subsection{Basic scheme}

Small amounts $(<10 \mathrm{nl})$ of neuroactive substances were pressure-injected via microcapillaries inserted dorsally into the brain. By the use of double-barrel electrodes two different substances were administered to the same location within the brain. Therefore, sequential injections of identical volumes of the same or of two different substances could in principle influence the same set of neurons. In some experiments mixtures of two or three substances (excitatory, inhibitory or modulatory) or different ions, dissolved in saline were used. To monitor the stridulatory behavior, the hindleg movements and the produced sound were recorded with two opto-electronic cameras and a microphone.

The animal is attached to a holder. The head is opened and the brain is

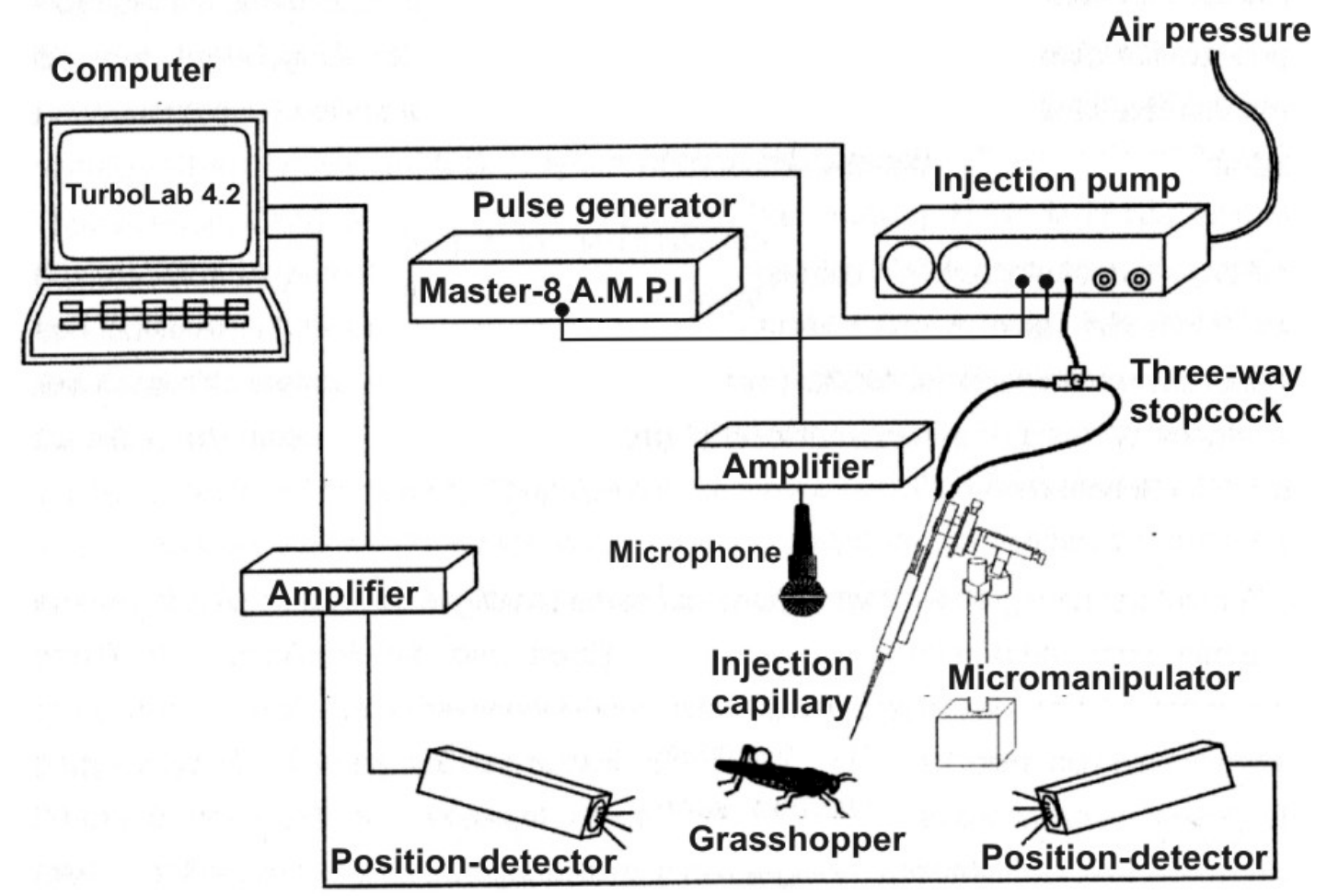

Fig. 2-1: Setup for pharmacological stimulation.

exposed. The microcapillary attached to the mechanic micromanipulator and is connected to the pressure-injection device through pressure resistant tubes. A three-way stopcock allows the pressed air to flow in two directions, respectively into one of the two barrels of the microcapillary. The pump is connected to a 
system that continuously supplies compressed air. Two optic-electronic devices (position detectors) record the stridulatory movements of the hind legs and transform them into a voltage signal. The latter is amplified and visualized on the computer screen and an oscilloscope. The signal is stored in data format ( ${ }^{*}$.dat) by the program Turbo Lab 4.2 or 4.3 for DOS.

\subsection{Animals}

Adult specimen of the gomphocerine grasshopper Chorthippus biguttulus (L. 1758) (Ch.b.) were caught in the vicinity of Göttingen, Germany, and kept in the laboratory for up to several weeks. Additional Ch. biguttulus were reared from eggs that were collected in the previous summer and kept at $4^{\circ} \mathrm{C}$ for $>4$ months. The nymphs hatched after $\sim 1 \mathrm{wk}$ at $26^{\circ} \mathrm{C}$ and were raised on wheat and supplemental food for crickets (Nekton, Pforzheim) at a 16/8 h light-darkcycle. All pharmacological experiments were conducted with male and female adults at room temperatures of $20-25^{\circ} \mathrm{C}$. Virgin females were separated as third or fourth stage nymphs kept in separation and used as adult for acoustic stimulation experiments.

\subsection{Preparation for pharmacological stimulation}

For pharmacological stimulation, the grasshoppers' pronotum was attached with wax to a holder, and the head was fixed to the pronotum. The front cuticle of the head capsule was opened with a razor blade to expose the dorsal surface of the brain. The rest of the animal was left intact and capable of moving all its appendages freely, particularly its hindlegs used in stridulation. To record the stridulatory movements with two opto-electronic devices (Helversen and Elsner 1977), a piece of reflecting foil (Scotchlite $3 \mathrm{M}$, type 7610; $2 \mathrm{~mm}$ diameter) was glued to the femur of each hindleg. The up and down movements of each hindleg were thus transformed into voltage signals proportional to the amplitude of movement. In addition, the sounds produced during stridulation were recorded by a custom-made microphone.

\subsection{Injection of drugs}

The neuroactive substances were injected into central nervous neuropile regions through glass borosilicate microelectrodes using a pressure-injection device (hydraulic pump, WPI, model 820). Double-barrel microcapillaries 
connected to a three-way stopcock allowed application of approximately the same amount of two substances at the same site in the protocerebrum. Before the experiment, the ends of the electrodes were broken under visual control to produce a tip diameter of approximately 10-15 $\mu \mathrm{m}$. The pressure and pulse duration delivered by the apparatus were adjusted so that approximately 1-10 $\mathrm{nl}$ of the substance was applied per injection. This had previously been confirmed by measuring the volumes of droplets injected into petroleum jelly (Hedwig and Heinrich, 1997).

A pulse generator Master-8 (A.M.P.I.) (Fig. 2-2) connected to the pressureinjection device (injectionhydraulic pump) was used to maintain accurately the parameters of the pressure

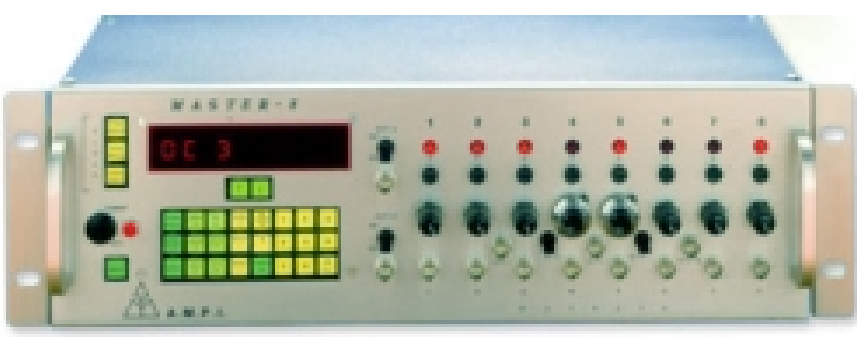

Fig. 2-2 Pulse generator Master-8 pulse (or trials of pulses) such as duration and the intervals in between the pulses. In some experiments complex trials of 5-6 pulses with different frequency were generated.

All experiments in this study were carried out using double-barrel capillary. The first chamber was always filled with a stridulation inducing substance, either proctolin or muscarine. The second chamber contained a test substance and the following protocols were used to study its effects on the previously induced stridulation:

Protocol 3-3-3 (all 9 pulses were injected at a fixed interstimulus interval $3 \min )$ :

a) to study a potential strong excitatory effect, the testsubstance was applied 3 times after a stable response to 3 injections of proctolin or muscarine was achieved. After that, 3 control pulses proctolin were repeated.

b) to study the role of co-transmission and a potential modulatory (enhancing or supressing) effect on the proctolin-induced stridulation the second chamber was filled with a combination of proctolin and 
the test-substance and injected 3 times. Three injections of proctolin alone both preceded and followed three injections of proctolin and the test substance.

Protocol 3-1-4 (the interval between all 7 injections of proctolin or muscarine was fixed, respectively 3 or $4 \mathrm{~min}$ ):

a) to study a potential inhibitory effect on the proctolin-induced stridulation 3 pulses of proctolin leading to a stable response were followed by 1 pulse of the test-substance, which was applied in between the $3^{\text {rd }}$ and $4^{\text {th }}$ proctolin pulses. After injecting the test-substance, proctolin pulses were continued until the duration of stridulation recovered values before its application.

The results of all conducted experiments were included into the statistical evaluation, except if one of the following disturbances occurred:

a) The proctolin pulses following the application of the testsubstance stimulated no stridulation or, compared with control injections in the beginning, stridulation with reduced duration.

b) Proctolin-stimulated stridulation was unstable with respect to latency and duration.

c) No test substance could be ejected from the capillary after its retraction from the brain at the end of the experiment. It can thus not be excluded, that the capillary was already blocked during the experimental injections.

\subsection{Drugs used for injection}

The neuroactive substances were usually dissolved in grasshopper saline (Clements and May, 1974) to give concentrations of $10^{-3} \mathrm{~mol}^{-1}{ }^{-1}$. $\mathrm{K}^{+}$ concentration in saline was adjusted to $5 \mathrm{mM}$ instead $10 \mathrm{mM}$ in Clements recipe. Acetylcholine, muscarine, proctolin, eserine, d-turbocurarine (dTC) neomycin obtained from Sigma-Aldrich; SQ 22536 obtained from Calbiochem; phorbol-12-myristate-13-acetate ( $\mathrm{PhE}$, porbol ester), thapsigargin, dopamine, glutamate, glycine, GABA, histamine, ivermectin (IVM), TMB-8, ryanodine, 
$\mathrm{PACOCF}_{3}, \mathrm{NiCl}_{2}, \mathrm{BaCl}_{2}, \mathrm{LiCl}$, purchased from Sigma-Aldrich were studied using upper described protocols.

Stock solutions of proctolin $\left(10 \mathrm{mM}\right.$ concentration in $\left.\mathrm{dH}_{2} \mathrm{O}\right)$ were preserved at $-20^{\circ} \mathrm{C}$.

Water-soluble substances were dissolved in grasshopper saline (Clements and May 1974) to give concentrations of 1 to $0,1 \mathrm{mM}$. A saline with lithium chloride $[40-60 \mathrm{mM}]$ substituted to $\mathrm{KCl}$ in some cases was prepared. Different ions, $\mathrm{Ba}^{2+}, \mathrm{Ni}^{2+}$ were added to the saline to give 1 to $5 \mathrm{mM}$.

Substances soluble in DMSO [thapsigargin, TMB-8, ryanodyne, obtained from Sigma-Aldrich] were dissolved in saline to give a $5 \%$ final content of DMSO. $\mathrm{PACOCF}_{3}$, soluble in ethanol, was dissolved in saline to give a $0,4 \%$ final concentration of ethanol. In these cases the same concentration of DMSO and ethanol was added to proctolin solution as a control. All solutions were preserved at $-4^{\circ} \mathrm{C}$ not longer than 7 days.

\subsection{Data processing}

The recorded signals were digitized on-line by means of an A/D-converter card (Real Time Devices AD3300) with the software Turbolab 4.2 (Bressner Technology, Germany) and stored as data files. The sampling rate for recording the stridulatory movements, the sound and the injection pulses was $5 \mathrm{kHz}$ per recorded channel. The software NEUROLAB (8.2, Hedwig and Knepper 1992; Knepper and Hedwig 1996) was used for visual examination and filtering of the original data.

One injection of a stimulating drug usually released several song sequences separated by short pauses. The time between stimulation pulse and the beginning of the first stridulatory sequence was determined as Latency. The sum of the durations of all individual song sequences released by one stimulation was calculated as the total sequence duration (S Duration) of stridulation. The duration from the beginning of the first sequence to the end of the last sequence was taken as the complete duration (C Duration) of stridulation.

The injected pulse, the beginning and the end of each stridulatory sequence were typed as numbers in a table of the calculating program Excel 
2000 (Microsoft). Latency and the durations of stridulation were normalized to the longest latency and the duration in that particular experiment (same volume of drug injected to the same site within the brain). The values were calculated and given in percent (\%). Normalization was necessary to enable comparison between different experiments with variable efficacy of proctolin stimulations, depending on the exact site of injection and the condition of the grasshopper. "Raw" diagrams were generated with Excel 2000 and subsequently imported into grafics program CorelDraw 9 (Corel Corporation) for assembly into composite figures and labeling. Original data (hind leg movements, pressure pulse) were exported from Neurolab 8.2. and subsequently re-assembled and labeled with CorelDraw 9. All figures were stored as grafics files $\left(^{*} . j p g\right)$ and imported into Word 2000.

\subsection{Evaluation and statistical analysis of experimental results}

Series of identical proctolin pulses, given at intervals of $3 \mathrm{~min}$ stimulate stridulation of initially gradually increasing duration that reaches a more or less stable value after the second or third pulse. This phenomenon has been described also for muscarinic activation (Wenzel et al., 2000). Stimulations in the initial phase before a conform stridulatory response was established, were not included into analysis.

When testing a substance for its capability to induce stridulation at a given site within the brain, experiments were defined as successful if stridulation was released by at least three successive injections, applied alone without any other inducing substances. When investigating putative inhibitory effects on proctolinstimulated stridulation, the average duration of proctolin-induced songs was calculated from at least three trials, executed at intervals of $3 \mathrm{~min}$. After application of a test-substance, experiments were defined as successful if the following two pulses of proctolin did not elicit stridulation or if the duration was below the range of two times the standard deviation (SD) of the average duration of proctolin-induced songs performed before inhibition, using protocol 3-1-4. The inhibition was classified as irreversible if the duration of stridulation never recovered into a range of two standard deviations again (Wenzel et al., 2000). The use of protocol 3-3-3 is described in section 2.4 and 3.3.2. Potential changes in the duration of proctolin-induced stridulation following the injection of 
a test-substance were evaluated by the nonparametric Friedman test (ANOVA) followed by the Wilcoxon-Wilcoxon multiple comparison test. This nonparametric test was chosen because all experiments were generally included into the statistical analysis, whether successful interference with proctolin stimulation occurred or not and therefore a normal distribution of the stimulated durations of stridulation could not be assumed. Kruskcal-Wallis ANOVA test that has been used previously to analyze mAChRs activation (Wenzel et al., 2000) was left out because all samples in all protocols described above were not independent. All statistical analysis was performed using the program KyPlot 2.0 (Koichi Yoshioka Software). 


\section{Results}

\subsection{Introduction to the natural acoustic communication of Ch.b.}

Acoustic communication in orthopteran insects has become one of the favorite subjects for investigations on the neuronal basis of invertebrate behavior. The stereotyped stridulation patterns, the relative ease of their elicitation, and the simplicity of the neuromuscular organization, which permits electrophysiological and pharmacological work in freely moving animals, favor both an ethological and a neurophysiological approach (Elsner, 1974).

The communication behavior of Ch.b. has been described several times under quasi- natural conditions in laboratory arenas (Helversen, 1972; Elsner, 1974), after incisions into the methathoracic ganglion (Heinrich, 1995) and elicited by electrical or pharmacological stimulations (Ocker and Hedwig, 1996; Ocker et al., 1995; Heinrich et al., 1997; Wenzel et al., 2002). The elements of song patterns have been described in various studies (Faber, 1928, 1929, 1932, 1953; Jacobs, 1953; Loher, 1957; Helversen, 1972). In this study the nomenclature given by Elsner (1974) is followed.

Under natural conditions the calling and courtship song is composed of short sequences, 3-4 of which form a sequence of $2^{\text {nd }}$ order. Usually, the first sequence of such a series lasts longer than the following ones (4-6 sec and 2-4 sec, respectively), with the first consisting of 60-100 chirps (Fig. 3-1), and the subsequent ones of 30-60 chirps (Elsner, 1974). Unlike Ch. mollis and O.v. the duration of the chirps does not increase during the course of the sequence. They may even decrease slightly from 70 in the beginning to $65 \mathrm{msec}$ at the end (Elsner, 1974). The chirps are well separated from each other by $10 \mathrm{msec}$ pauses during which the leg is kept in the upper (initial) position. This position is raised from chirp to chirp during the initial period and then kept constant for the rest of the sequence (Elsner, 1974). Each up- or down-stroke strictly corresponds to the one syllable within a chirp (Fig. 3-2). 


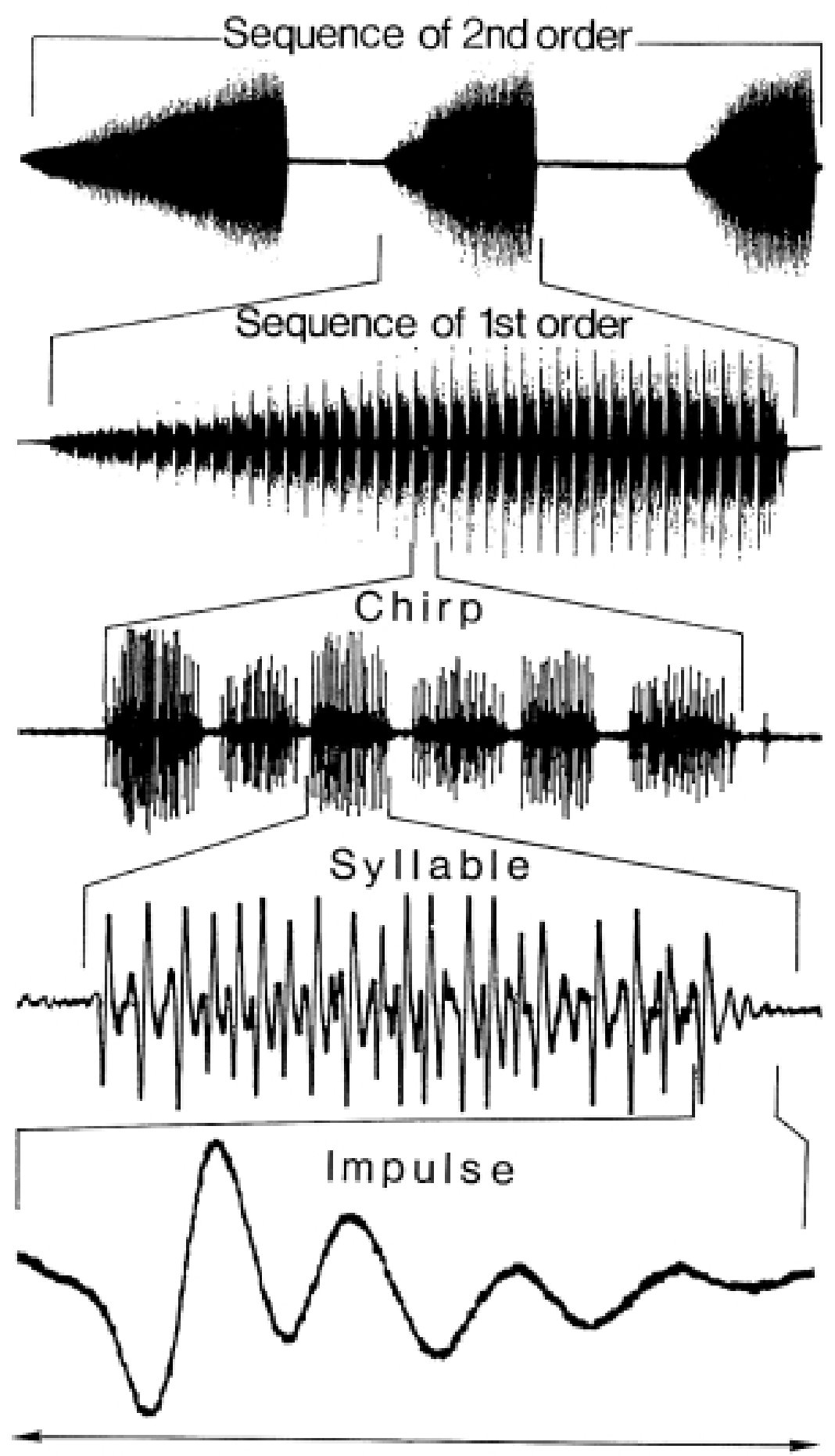

Fig. 3-1 Structure and terminology of gomphocerine song - Chorthipus biguttulus (Ch.b.)

Time mark ( $\longrightarrow$ ): $15,0 \mathrm{sec}$ (sequence of $2^{\text {nd }}$ order); $2,9 \mathrm{sec}$ (sequence of $1^{\text {st }}$ order); $72,5 \mathrm{msec}$ (chirp); 9,1 msec (syllable); 0,9 msec (impulse) (from Elsner, 1974). 
The stridulatory patterns of the left and right hind leg are strictly correlated and fundamentally the same, but they differ in some details from each other. A considerable phase shift between the left and the right hind leg has been observed in intact animals. That was due to prolonged last syllables (extended upstroke) performed by one leg, that fill the breaks between the chirps. Thus two intrinsic patterns have been described that were independent of the removal of the tegment. This indicates

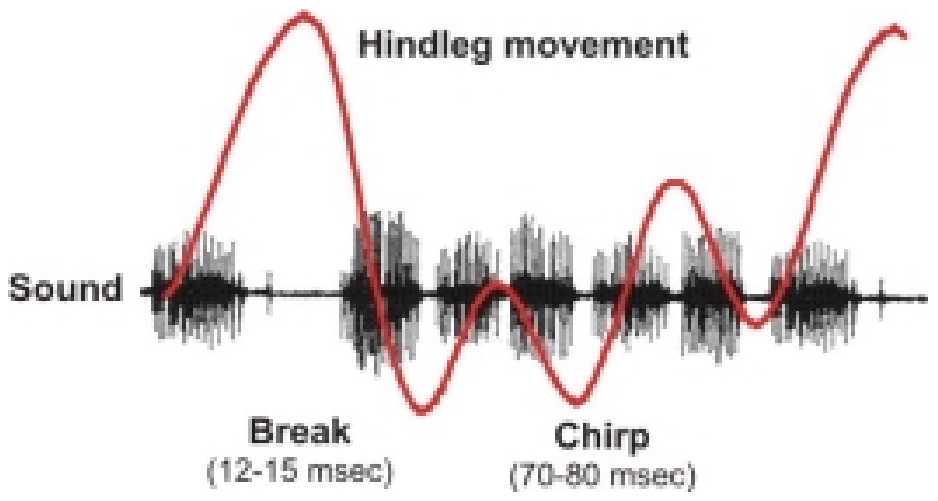

Fig. 3-2 Scheme of a strict corelation between hindleg movements and the sound production in Ch.b. male.

Each up- and down-stroke produces a syllable within a chirp. The break between the chirps is due to an up-stoke with higher amplitude. The break/chirp ratio is constant $(\sim 1: 5$ for chirps consisting 6 syllables, for example) under given conditions. that their production does not depend on sensory feedback (Elsner, 1974): pattern I - separated chirps with constant chirp/pauses ratio (Fig. 3-2); pattern II - a phase shift of the movements and an extended last upstroke fills the gaps between the chirps, produced by the (pattern I)-performing hindleg. After removal of one hindleg, the remaining one performed only the stridulatory pattern I, and never changed to pattern II. The appearance of two different movement patterns and their coordination depends on commissural connections within the $3^{\text {rd }}$ thoracic ganglion complex (Heinrich and Elsner, 1997). The distribution of pattern I and II between the hind legs may change every few sequences in fully intact grasshoppers.

Calling and courtship songs of Ch.b. are very similar. They differ from each other only in the amplitude of the upstrokes, the sound intensity (Reis, 1995) and the duration of the sequences $1^{\text {st }}$ order (Ocker, 1995). Apart from these slight differences, the most reliable factor to distinguish between these song types is the presence or absence of a female.

A male in search of a female performs calling song (sequence of $2^{\text {nd }}$ order) at almost regular time intervals (every $30-60 \mathrm{sec}$ ). A female ready to mate re- 
sponds to the song, but because competition for females is high among the males, it is up to the male to localize the female and to take the risk of approach. Upon hearing a female response, the male turns abruptly towards the side from which the female's signal comes. It moves forward a short distance $(5-15 \mathrm{~cm})$, sings again performing courtship song (shorter sequences), and turns anew after the next answer of the female. Thus, the male approaches in a stepwise manner, making successive decisions (von Helversen, 1997) (Fig. 3-3). The turning angle is usually larger than that needed to bring the sound source in a lateral position on the previously sound-contralateral side. This behavior, termed laterization, results in a zig-zag course of the approach. Only when a female response is perceived in the frontal auditory field, the male may react by jumping straight forward towards the sound source (von Helversen, 1997).
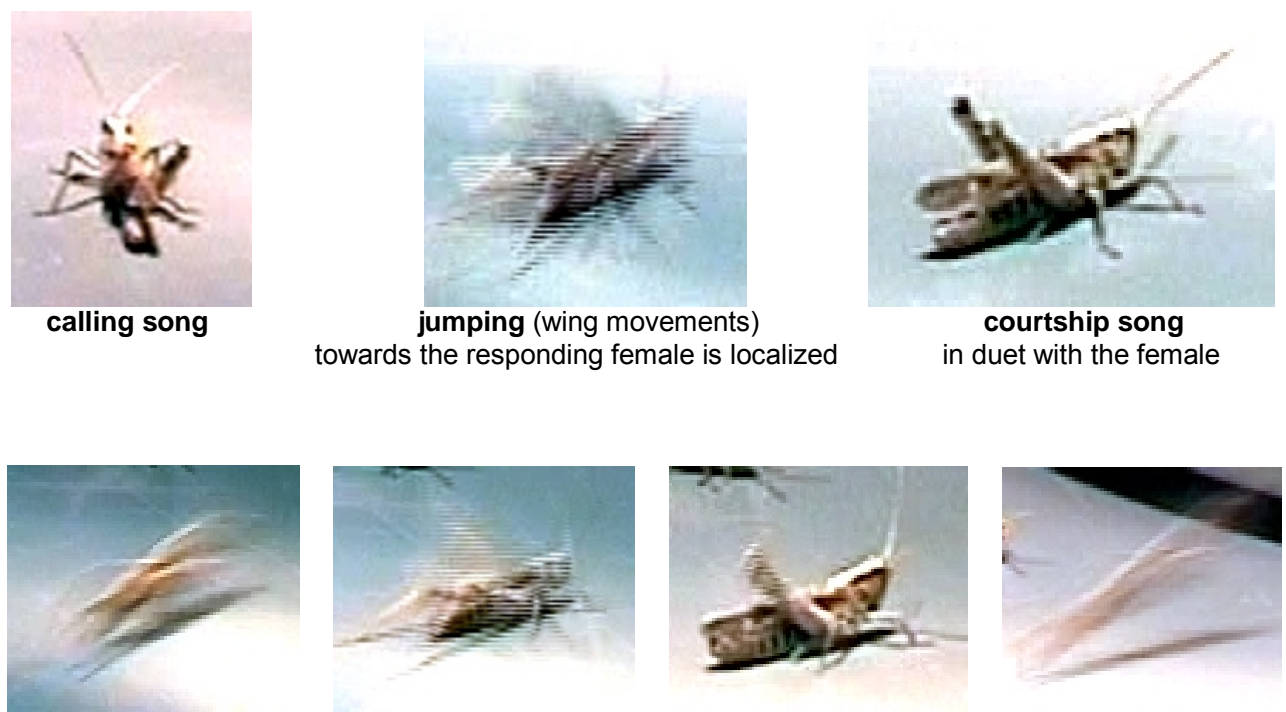

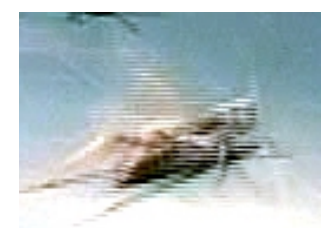

landing (wing movements)

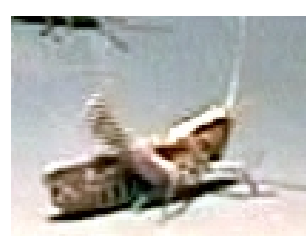

courtship song

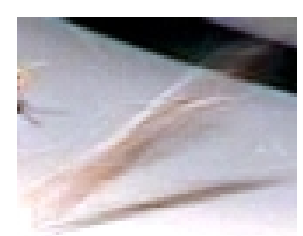

jumping

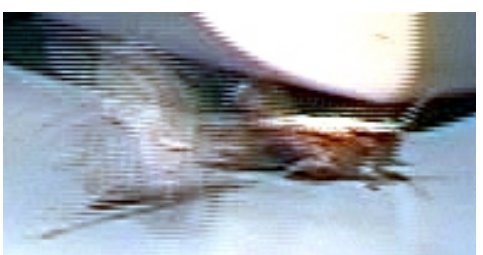

the stridulating female is reached

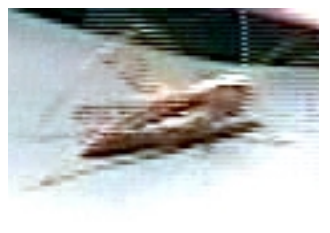

landing

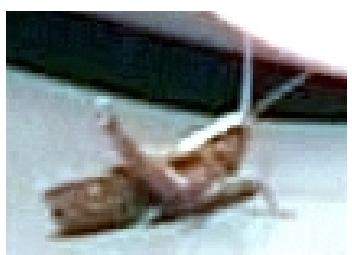

calling song

Fig. 3-3 Phonotactical mate approach of male (Ch.b.) in response to female stridulation. Consecutive performance of leg stridulation, jumping and wing movements. 
Under natural conditions the rivalry song of Ch.b. has not been clearly described. The most distinguishing features compared to calling and courtship songs might be the amplitude of the upstrokes and considerable louder initial sound intensity (Reis, 1995).

On the other hand, the females perform specific courtship acts in response to the male songs (Fig. 3.4, personal observations):

1. Stridulation - corresponds to high level of excitation;

2. Wing flappings - performed always without stridulation;

3. Phonotaxis - active approach to the sound source;

4. Lateral orientation of the longitudal body axis and typical shifted position of the body and the hindlegs - one hindleg from the sound source side is kept in down position and the second leg in up-position;

5. "Kicks" performed by the hindlegs and/or lifting them in a specific manner;

6. "Cleaning" the antenna (Antenna putzen);

7. Opening the abdomen followed by kick;

8. Others. 
Fig. 3-4 Females (Ch.b.) perform following courtship acts induced acoustically:

\section{Stridulation}
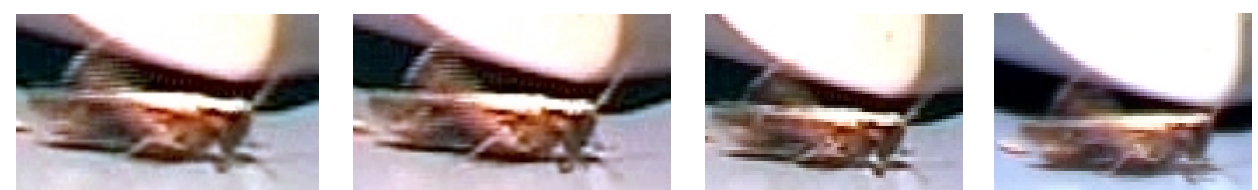

2. Wing flapping performed without stridulation
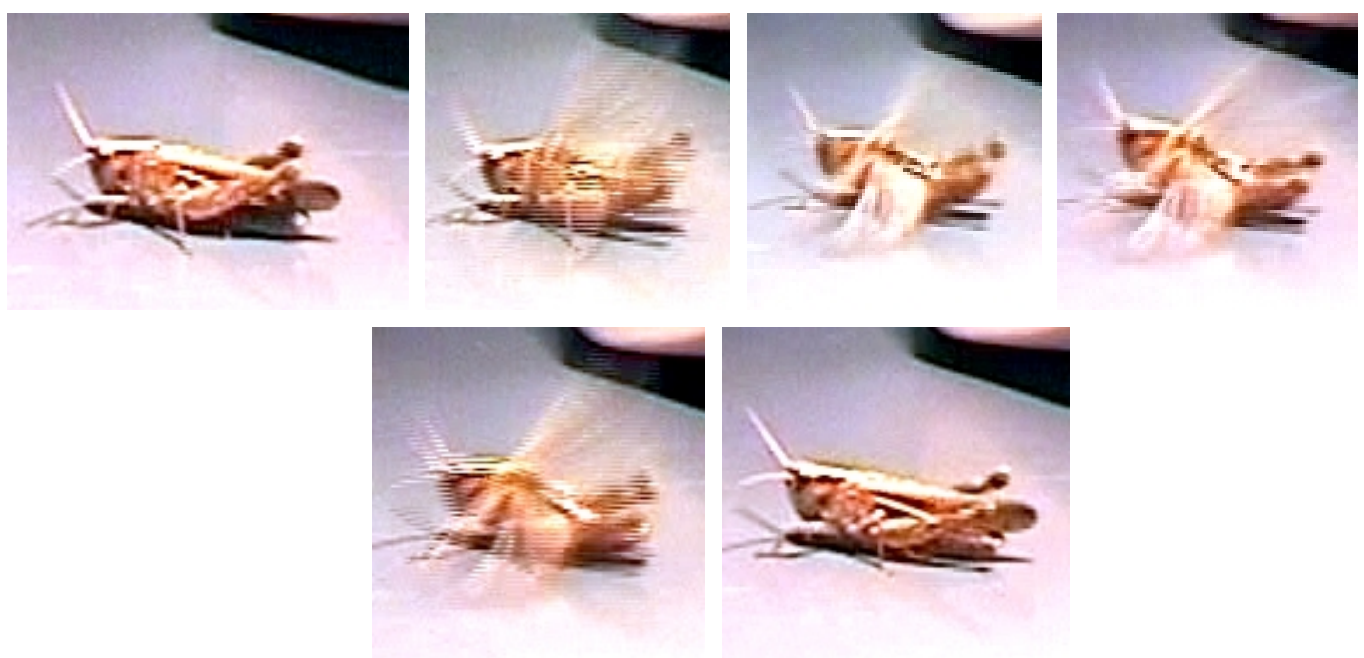

\section{Phonotactical approach (phonotaxis)}
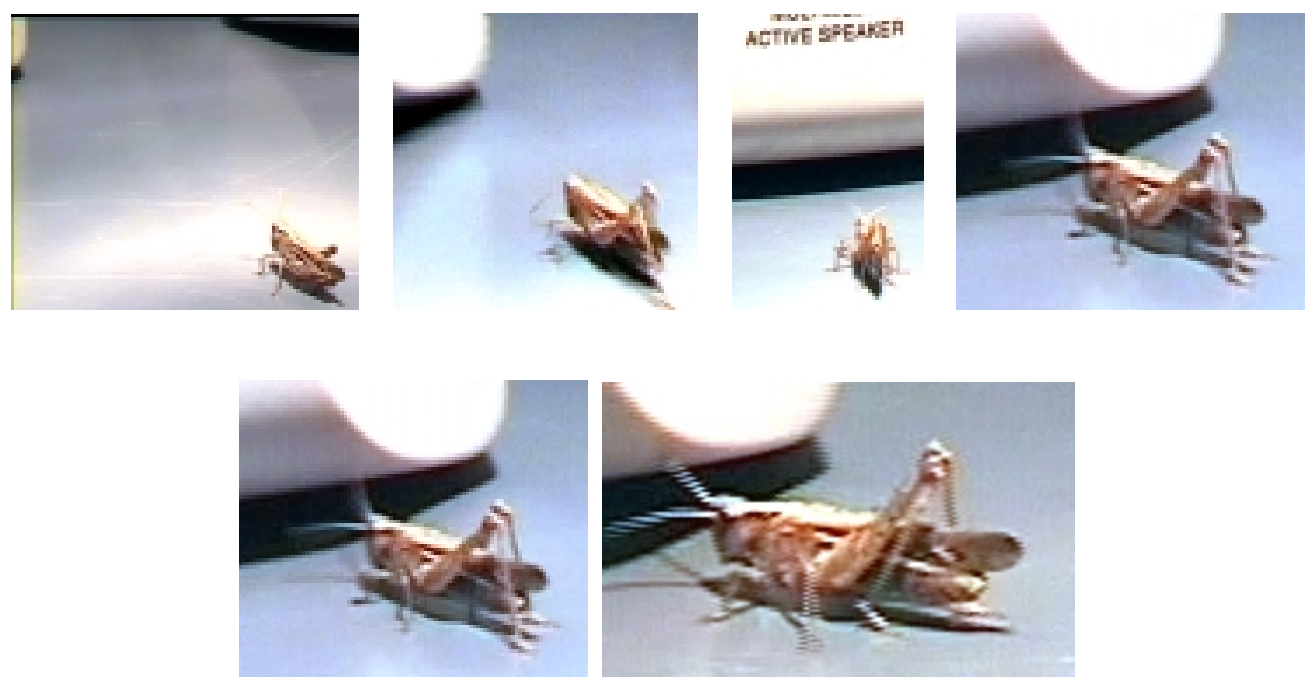

4. Lateral position of the longitudal body axis to the sound source
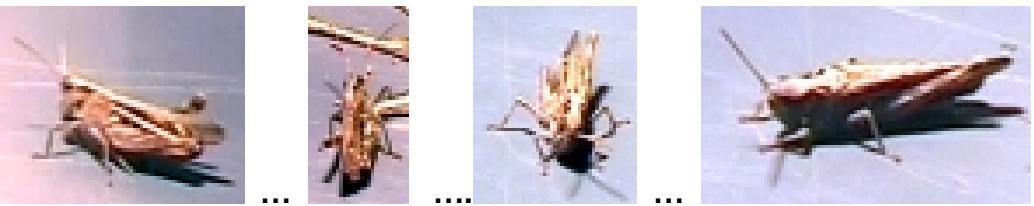
5. "Kicks" with the hindlegs and lifting them in a specific manner
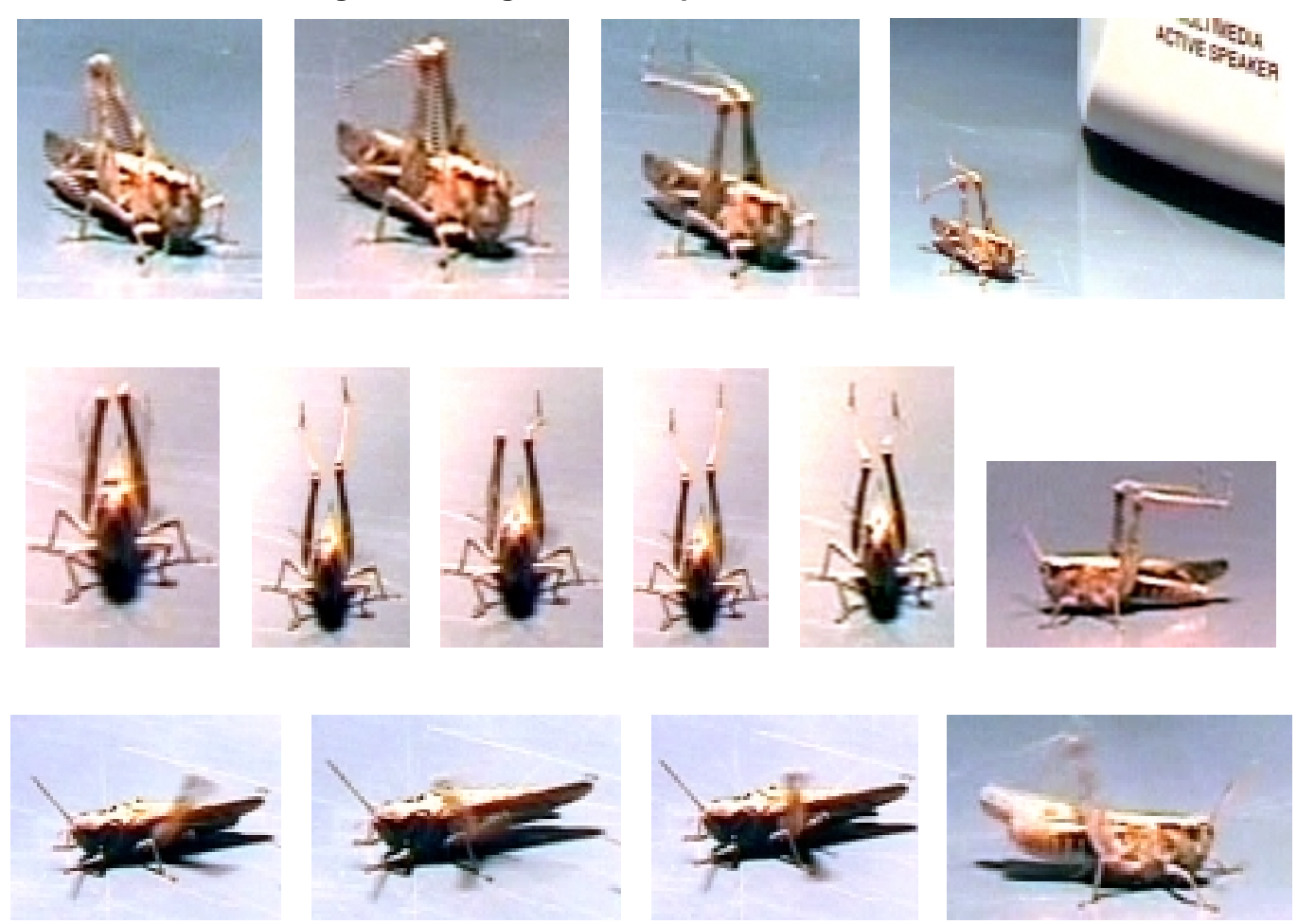

6. „Cleaning“ the antenna (Antenna putzen)
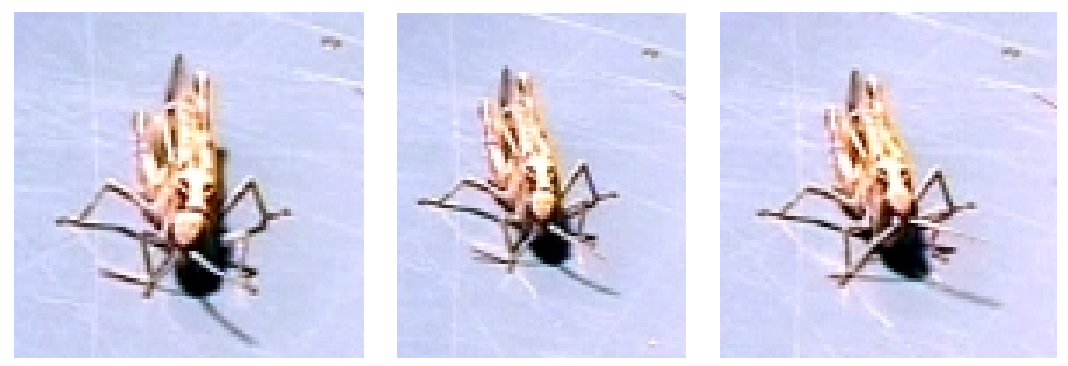

7. Opening the abdomen following by kick
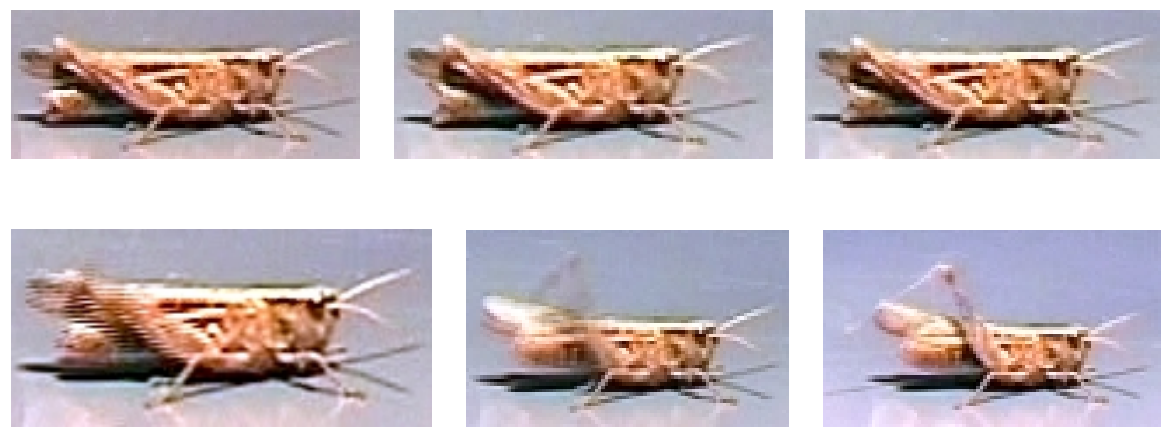

8. Others - female touches the ground with the antenna.

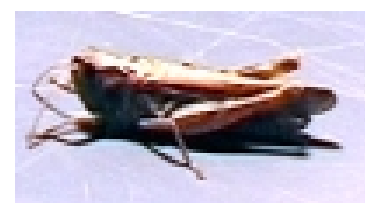




\subsubsection{Short introduction to the anatomy and physiology of the sound production in the subfamily Gomphocerinae}

Insect brain, as well as the ganglia, is composed of a central core of nerve cell axons and their branches, surrounded by a rind of peripherally dispersed cell bodies. A consequence of this organization is that most insect neurons are structurally unipolar. The brain core consists of a series of longitudal tracts and horizontal commissures representing the axonal lengths of neurons surrounded by a tangled web of branches, the neuropil (Mobbs, 1985). Despite its spaghetti-like appearance, tangled neuropil is highly ordered and patterned in term of architecture of its individual constituent neuron geometries. The brain differs from the thoracic ganglia in that a large part of its neuropil volume is overtly patterned. This pattern results from the repetition of the same intraganglionic interneuron morphologies (Mobbs, 1985).

The function of the brain of the grasshoppers is primary focused around receiving and processing sensory information from the various organs of the head, and the seventh and eight ventral gandlia (Chapman, 1969).

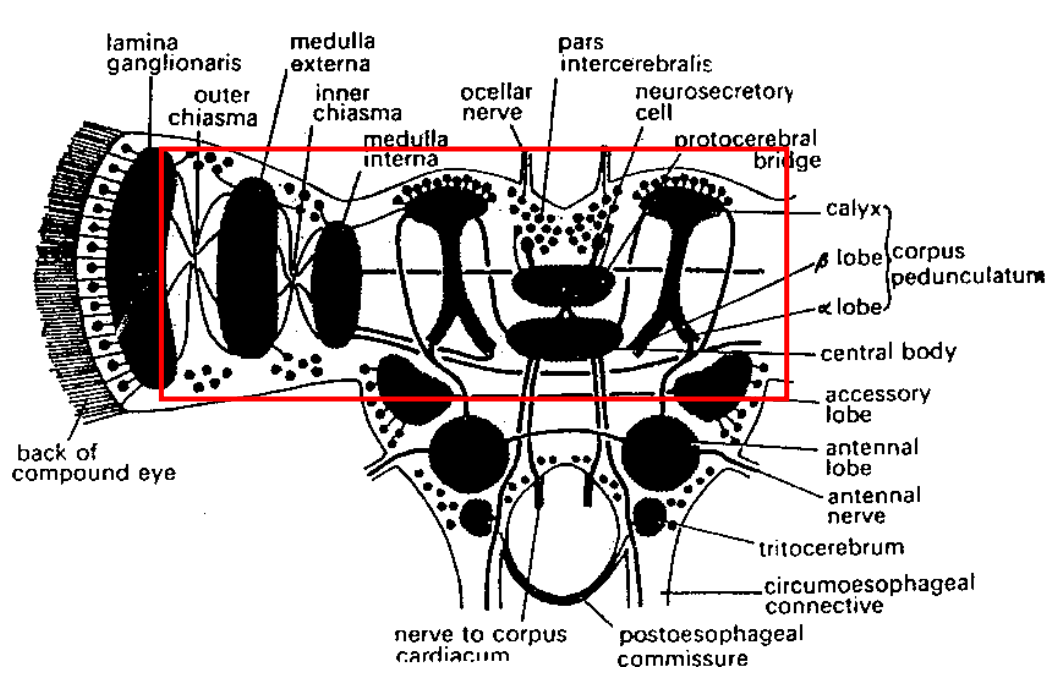

Fig. 3-5 Generalised insect brain - major areas neuropile, tracts and somata. Reproduced from Chapman (1969)

The brain has direct motor control of the antennae, some movements of the head and certain aspects of locomotion (decapitation results the loss of the ability to walk backwards (Wigglesworth, 1972). In addition, the brain integrates ascending and descending information, which then is used to influence via the pre-motor descending interneurons, the motor output of other ganglia (Chapman, 1969; Huber, 1974). In this way, the brain is able to heighten or suppress certain reflex reactions. 
The protocerebrum (Fig. 3-5, red rectangle) is the main area of the brain, it is bi-laterally symmetrical and has been sub-divided into numerous neuronal tracts, commisures, and neuropil areas. Some of the most documented include the mushroom bodies (consisting of the calyx and the pedunculus) the central complex, the lateral accessory bodies and the optic lobes. The functions of the protocerebrum include:

- mushroom bodies - processing of olfactory information in the calyx (Ernst et al., 1977; Erber, 1980), production of entire and complex patterns (selecting and coordinating behavior in crickets) (Huber, 1955; Elsner and Huber, 1969; Otto 1971; Wadepuhl and Huber, 1979 etc.).

- central complex and optic lobes process visual information (Mobbs, 1985)

- accessory bodies integrates mechanosensory information (Burrows, 1996) also there auditory information (Boyan et al., 1993).

This is a very simplified view of the workings of what is probably one of the most complex part of the grasshoppers brain. For full reviews of the protocerebral interconnections and function see Williams (1975), Mobbs (1985) and Boyan (1993).

Species of the subfamily Gomphocerinae produce species-specific sound patterns (courtship, calling and rivalry song) for intraspecific communication by rubbing the hindlegs against a cuticular vein of the forewings. These hindleg movements are genetically and centrally programmed and are regarded as a typical example for a fixed action pattern (Elsner and Huber, 1969). The neuronal network underlying the motor commands for stridulatory leg movements is housed in the methatoracic ganglion complex (Gramoll and Elsner, 1987; Hedwig 1986a, 1992a, b). This complex consists of two hemiganglionic pattern generators (Ronacher, 1989). These are connected via local bilaterally arborizing interneurons and thus produce coordinated phase-coupled hindleg movements during stridulation (Hedwig, 1992a).

Evidence has been accumulated that the performance of stridulatory behavior is under control of the brain. Stridulation in grasshoppers can be induced by small tissue lesions within the brain and can be reliably elicited by local electrical stimu- 
lation of certain brain areas (Elsner and Huber, 1969; Hedwig, 1986a; Huber 1955; Wadepuhl, 1983). Male and female grasshoppers will respond to the acoustic signals of the other sex only if the perceived sound patterns match their innate releasing mechanism. The corresponding auditory filter mechanisms are located within the brain (Bauer and von Helversen, 1987). As a consequence there must be a descending cephalothoracic pathway by which the brain controls stridulatory behavior and activates the thoracic pattern generators whenever stridulation is to be performed (Hedwig, 1994).

It was postulated (Wiersma and Ikeda, 1987) that the brain of invertebrates might control coordinated movements or activate pattern-generating networks by tonic activity of specific sets of command interneurons or systems of functionally uniform command elements. This concept of command neurons has received considerable attention. Strict criteria (sufficiency and necessity) for the identification of particular neurons as command neurons have been given and formulated by Kupfermann and Weiss (1978). Descending neurons controlling stridulation in the grasshopper Omocestus viridulus (O.v.) have been identified and found to fulfill the criteria of sufficiency and necessity. The so called B-DC-3, B-DC-4 and B-DC-5 (B - brain; DC - descending, contralateral) neurons have been intracellularly recorded and stimulated and anatomically identified with staining techniques (Hedwig, 1994; Hedwig and Heinrich, 1997). The occurrence of stridulatory behavior has been strictly coupled with tonic activity in the B-DC-3,4,5 interneurons. They have been demonstrated to be sufficient to initiate and maintain the speciesspecific leg movements of ordinary stridulation (B-DC-3), hindleg shaking (B-DC-4) and precopulatory movements (B-DC-5) (Hedwig and Heinrich, 1997). The axons of all three types of command neurons project through the medial portion of the connectives and are suggested to make direct connections with the thoracic pattern generating circuits (Hedwig, 1994; Heinrich, 2002). They cross the mid-line of the protocerebrum and exhibit a profuse arborization pattern within the medial dorsal protocerebral neuropil. 


\subsubsection{Pharmacological stimulation of stridulation in Ch.b.}

Previous studies have demonstrated a role for ACh and both nicotinic (nAChRs) and muscarinic (mAChRs) receptors in the cephalic control of the singing behavior of various grasshopper species (Ocker et al., 1995; Hedwig and Heinrich, 1997; Heinrich et.al., 1997, 2001a, 2001b; Wenzel, 2002). It has also been reported that long lasting stridulation could be elicited through disinhibition of the cephalic control circuits with the $\mathrm{Cl}^{-}$-channel blocker picrotoxin (Heinrich et al., 1998). Preliminary studies have also suggested, that proctolin could serve as a sufficient stimulant for the pharmacological initiation of stridulation in O.v. (Heinrich, 1995; Plettenberg, 1998). Whether proctolin could also activate specific singing behavior in other species with more complicated stridulatory rhythms and how proctolin might mediate its stimulatory effects on the control circuits, has not been tested.

The aim of this study was to characterize the functional role played by proctolin in the cephalic control of stridulation in Ch.b. and a description of the cellular mechanisms that underlie its contribution. 


\subsection{Proctolin elicits singing behavior in males of Ch.b.}

Injections of proctolin into the frontal part of the central complex and an adjacent neuropil anterior and dorsal to it elicited the entire repertoire of the speciesspecific singing behavior in males of Ch.b. (Fig. 3-6) Calling, courtship and rivalry songs could be released as well as some additional elements of the sexual behavior that will be presented in a later section. The effect of proctolin was very sensitive to the accurate position of the capillary. Moving the electrode by only a few $\mu \mathrm{m}$ usually caused the stimulatory effects of proctolin to appear or to vanish.

In most experiments stridulation started after a short latency (3-15 sec) and lasted

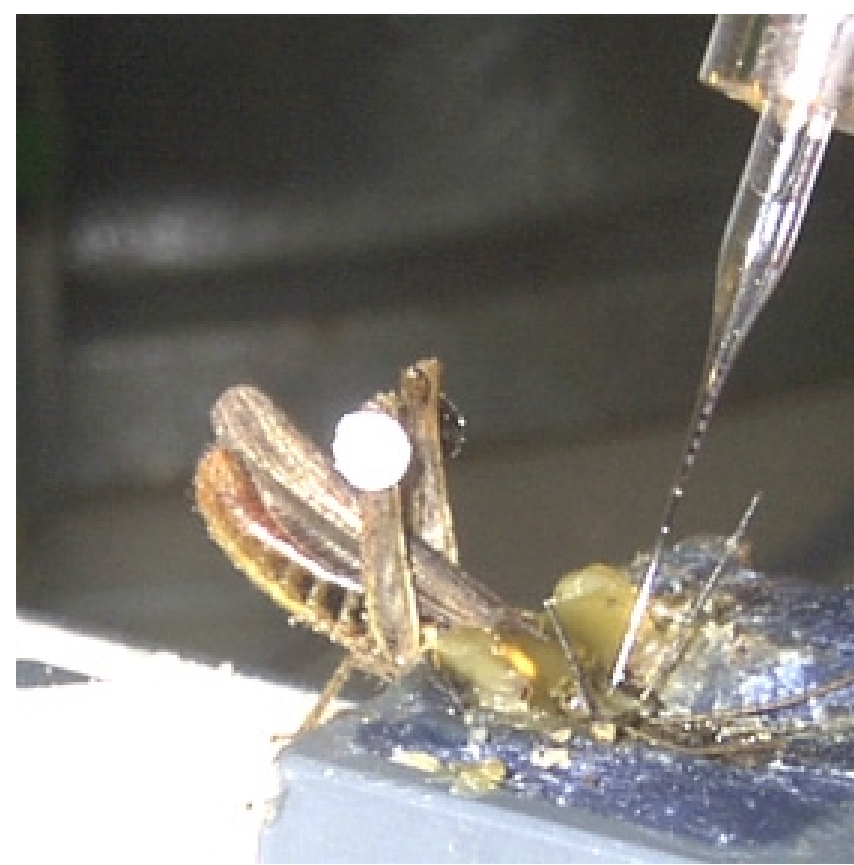

Fig. 3.6 Proctolin injected by microcapillary into the brain of male grasshopper (Ch.b.) stimulates stridulation. for 4-30 sec. Rarely the very first stimulation pulse applied to a particular site within the protocerebrum elicited stridulation. In most experiments overt behavior could only be elicited after the $2^{\text {nd }}$, $3^{\text {rd }}$ pulse. In this initial phase of the stimulation the first pulse that elicited stridulation did this after a longer latency than later pulses and the duration of response was shorter. 

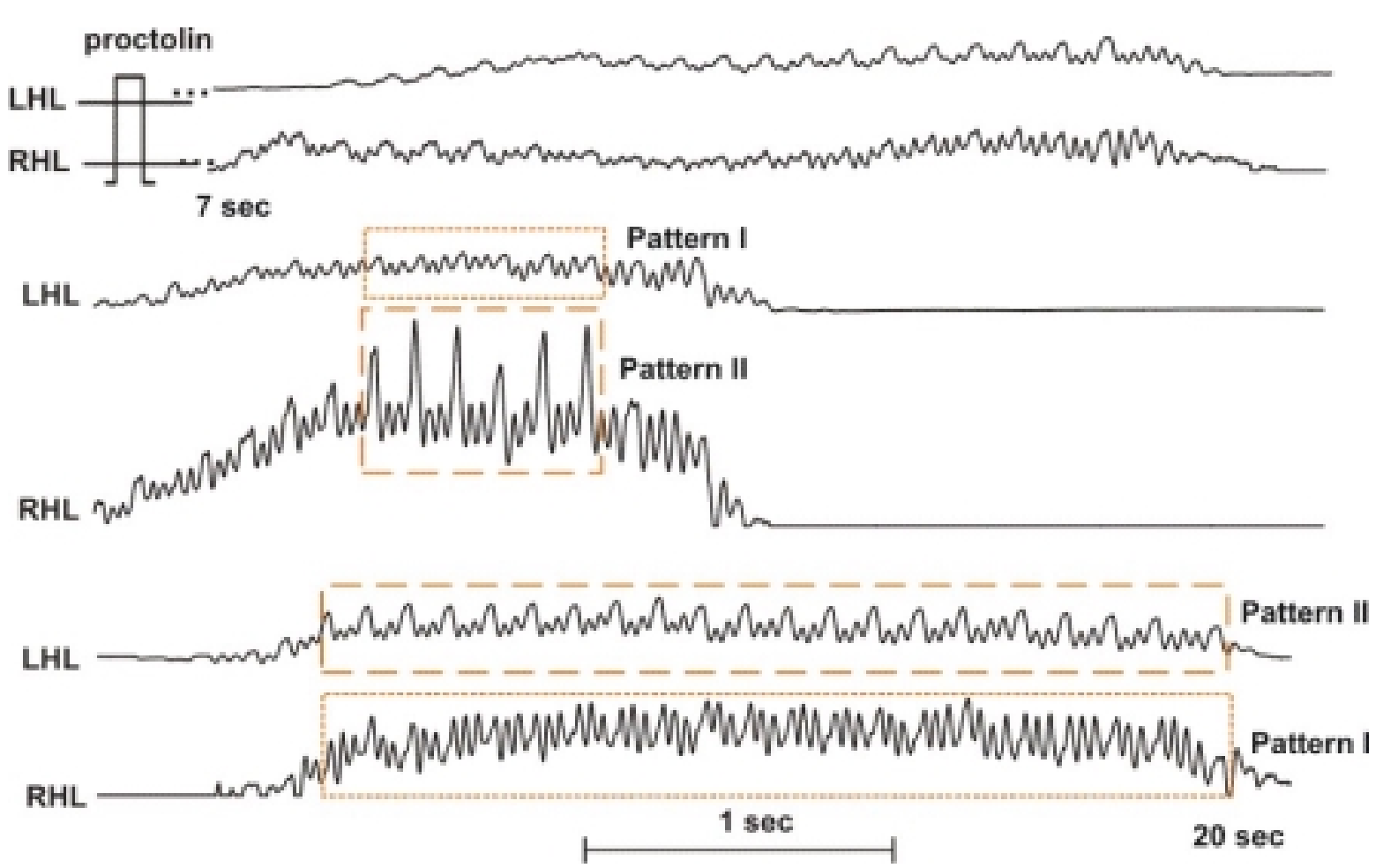

Fig. 3-7 Song sequences of male Ch.b. induced through a pulse of proctolin injected into the protocerebrum. Patterns I and II (red rectangles) were performed alternatively by both hindlegs.

The following marks are used in all figures, containing stridulation:

RHL - right hindleg; LHL - left hindleg; 7 sec - the latency of the response (the proctolin pulse is taken as 0 ); $20 \mathrm{sec}$ - the end of the song; $1 \mathrm{sec}$-time scale.

Similar to what has been observed under natural conditions the proctolinstimulated male courtship song of Ch.b. was composed of one or two longer sequences of $1^{\text {st }}$ order $(3-5 \mathrm{sec})$ followed by shorter ones $(1-3 \mathrm{sec})$, the longer sequences consisting of 40-80 chirps and the subsequent ones of 10-40, respectively. The sequences of $2^{\text {nd }}$ order contained $2-6$, in some cases up to 15 sequences of $1^{\text {st }}$ order. Fig. 3-7 shows a song with three sequences of $1^{\text {st }}$ order. In most experiments both movement patterns were observed, one leg performed pattern II with an extended last upstroke after the last syllable of the chirp and the other performed pattern I with smaller up- and down-strokes movements and a resting period at the upper reversal point (Fig. 3-7, red rectangles). Every few sequences, the patterns exchanged between the hind legs.

Up- and down- movements of the hindlegs produce the typical chirp pattern of the courtship song. Proctolin stimulation typically elicited chirps consisting of 6 in- 
A

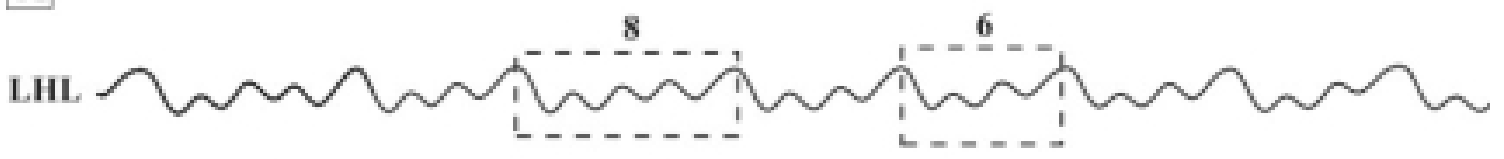

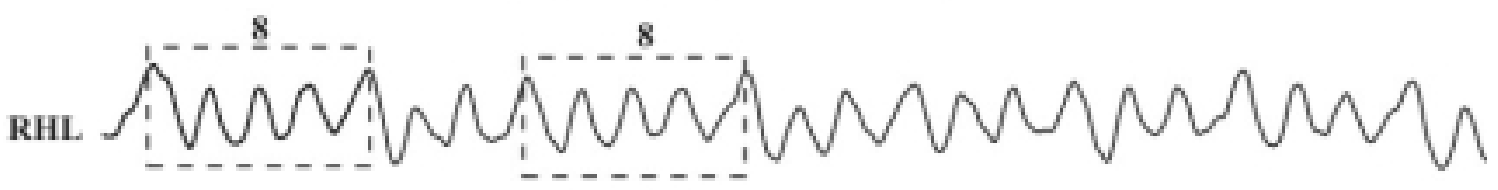
B
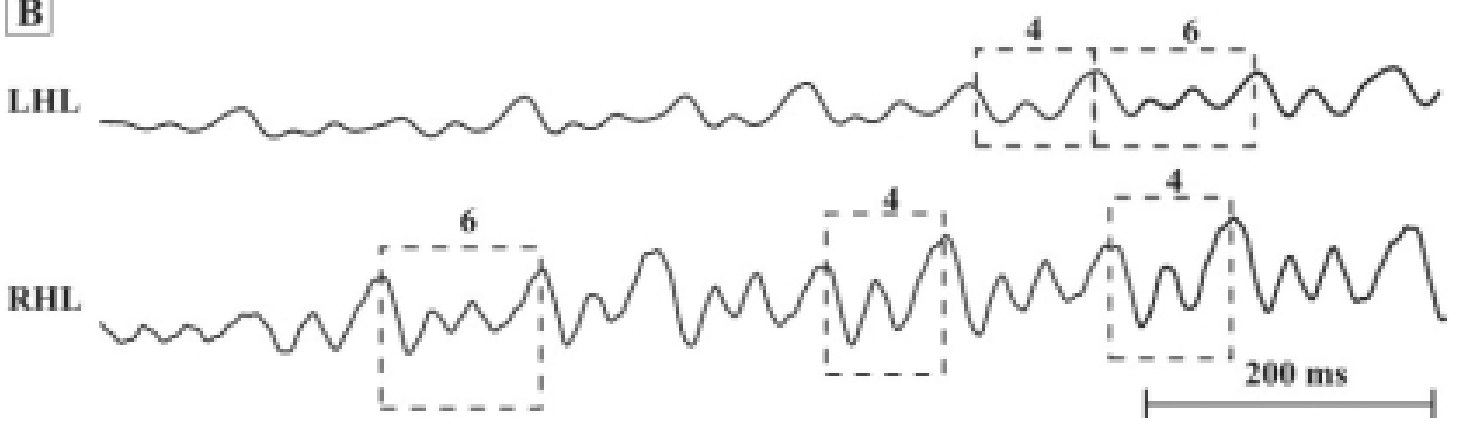

Fig. 3-8 Different number syllables in the internal chirp structure of the male song Ch.b., induced by proctolin. Up- and down-strokes movements produce a particular syllable in the chirps of the male courtship song. Chirps containing 6, 8 (A) and 4, 6 (B) syllables are shown.

dividual syllables corresponding to two small-amplitude and one largeamplitude up- and down-movement. The number of the syllables per chirp of the male song of Ch.b. could vary.

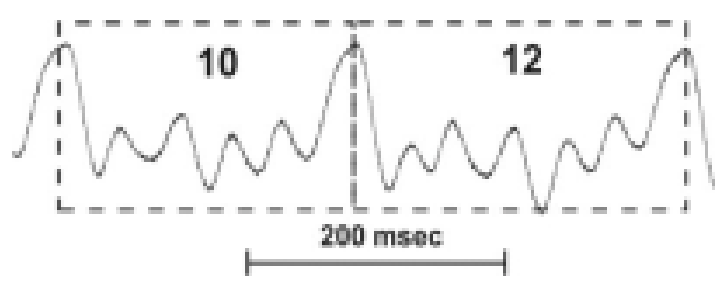
Chirps with 4, 6, 8, sometimes 10-12 (right) syllables per chirp were obtained examining the effect of test substances on proctolin-induced stridulation. The number of the syllables, the internal frequency of the up- and down-strokes has been reported to depend on the temperature (von Helversen, 1972). All pharmacological experiments reported here were carried out at room temperature 20-22 C.

Calling and courtship male songs differ in Ch.b from each other only in the amplitude of the upstrokes, the sound intensity and the duration of the sequences $1^{\text {st }}$ order (Ocker et al., 1995; Reis, 1995). Therefore, a distinction between callingand courtship stridulation was not attempted for proctolin-stimulated songs. But in the light of later results, examining the modulation of proctolin mode of action by 
different transmitters, an evidence of additional differences and characteristics between calling and courting song are discussed.

In some experiments, proctolin elicited stridulatory movement patterns that resembled those performed during rival songs of male Ch.b. The features proctolin-stimulated rival songs were short sequences (1-2 sec), with large initial up- and down strokes (Fig. 3-9, $5^{\text {th }}, 6^{\text {th }}$ and $7^{\text {th }}$ sequences). After initiation calling song it turned into rivalry song. In the experiment shown in Fig. 3-9, proctolin induced 7

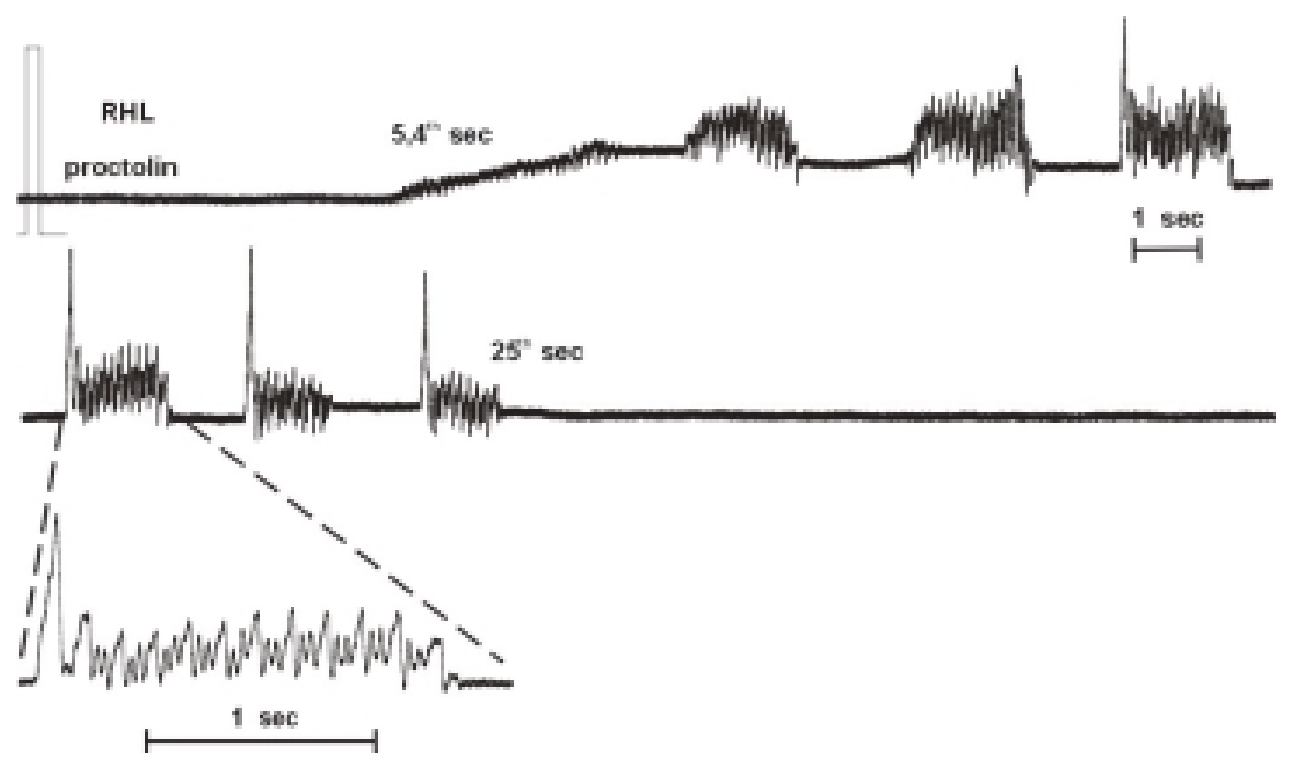

Fig. 3-9 Rivalry/Courtship sequences produced by male Ch.b. by proctolin stimulation.

song sequences of $1^{\text {st }}$ order. The first one was longer than the others and corresponded to the initial phase of the singing (see 3.1). The next two sequences represented the calling song, while the last 3 possess the features of rivalry song described by (Ocker et al., 1995; Heinrich, 1995; Wenzel, 2000). The $4^{\text {th }}$ sequence owns the properties of both normal and calling song. Transitions between song types (e.g. calling into rivalry) occur often, but in the beginning of this study this was not considered. Only in few experiments were observed short sequences with initial high sound intensity that decreased gradually. In most experiments, as in this shown in Fig. 3-9, the shorter sequences (1-3 sec) occurred without this typical for rival song vigorous loud sound intensity. This arouse the question whether the stimulated song pattern fit to the natural repertoire or the definition of the rivalry song overlaps with the features of courtship song because their sequences have 
been also reported to be shorter (Ocker, 1995). Rivalry song in freely behaving males is uncommon. The possible overlapping of the features of rivalry and courtship songs is discussed later.

\subsubsection{Other proctolin-stimulated elements of sexual behavior (courtship)}

The sexual behavior of orthopterans consists of many complex actions involving sound production, typical movements and positioning of the body, which are performed by males and females in preparation for and during courtship. Many details of gomphocerine courtship elements have been described by the early classic studies of Jacobs (1953) and Faber (1953).

Some of these courtship elements were also stimulated by proctolin. Some of them were recorded by digital video camcorder (Canon MV10i), but were not evaluated. Most of these could not be recorded, since only sounds and hindleg movements were monitored in our experiments. Moreover, these courtship elements only appeared occasionally in some experiments and their release could not be associated with a particular site of proctolin injection or any other stimulation parameters.

Under natural conditions the males perform the calling song followed by courtship song if the female shows a sign of copulatory readiness (female song or active phonotaxis).

As soon as the female shows copulatory readiness the male from O.v. ends his song with sharp "tick"-sounds (Elsner, 1974) that initiate an attempt to copulate with the female. This pattern has been well described in O.v., where these "tick"sounds, later called precopulatory movements (Hedwig, 1995), represent a particular pattern within courtship songs of high intensities, usually performed at the end of the courtship.

In Ch.b. precopulatory movements have been described in very early works (Jacobs, 1953). Similar patterns were also released through injections of muscarine into the protocerebrum of Ch.b. performed by Wenzel (2000). 
In my studies, combination of proctolin and glutamate occasionally elicited precopulatory hindleg movements that were either performed exclusively (section 3.6.4) or connected to a regular song sequence.

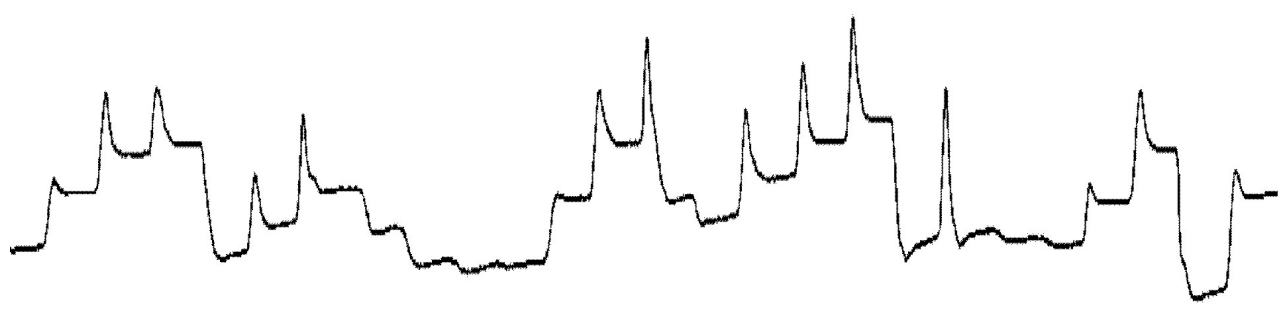

Fig. 3-10 "Tick" sounds producing by precopulatory movements following or preceding a courtship song sequences of Ch.b. induced by co-injection of proctolin and glutamate (see section 3.6.4)

Under natural conditions the courtship song, as it was described before (3.1), is preceded and followed by orientation of the male's longitudinal body axis lateral to the responding female with a slight slope of the body in contra-lateral direction with typical HLs positioning - the HL from the sound source side is in down position ("exposing the ear") and the other one in up-position (section 3.1, Fig. 3-4(1)].

Interestingly in certain experiments such positioning the HLs with the described slope of the body was induced through proctolin stimulations. Unfortunately, only at the end of this study was paid attention of that and such data was not evaluated.

Another courtship element, described in other species and in the female repertoire (Ch.b) is the kicks, done by the HLs or the HLs slowly lift up, in a specific manner [Fig. 3-4(5)]. In some stimulations such positioning of the HLs were performed for 10 $30 \mathrm{sec}$, indicating that it might be elicited pharmacologically (Fig. 3-11).

Usually females (Ch.b.) to show copulatory

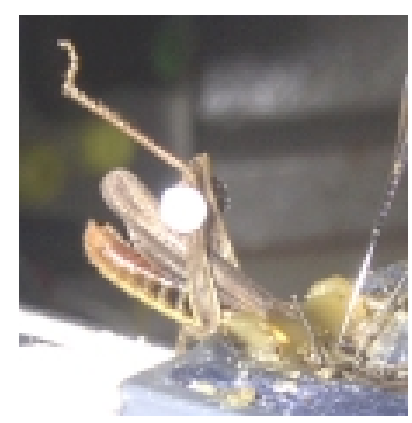

Fig. 3-11 Pharmacologically induced slow lifting up of the hindleg with trembling tarsus (male, Ch.b.) readiness respond to courtship male song either with female stridulation, other typical movements (touching the ground with the antenna, turning the body axis 
lateral to the sound, kicking both hind tibiae simultaneously backwards etc. or doing phonotaxis. In some case to show copulatory readiness they did rapid wings movements - thickened veins of the hind wings touch each other at the upper reversal point. This is called wing flapping [(Fig. 3-4 (2)]. It is typical for another species with fast stridulatory movements and complex singing patterns Stenobothrus rubicundus (GERMAR 1817), where the male courtship song usually ends with wing stridulation. The females of St. rubicundus (Greek population) respond to the male songs only with wing stridulation and not leg stridulation (Elsner et al., 1995).

Surprisingly in 4 experiments in response to combination of proctolin pulses injected into the brain of males Ch.b. was recorded the wing stridulation following the courtship song. Such a phenomenon has been not described in Ch.b. males under natural conditions.

A possible involvement of proctolin in a hypothetic switch mechanisms between flight and stridulatory control systems could not be excluded (section 3.6.4)

All additional elements described in this paragraph only appeared in connection with stimulated stridulation. None of them could be stimulated exclusively (with the exception of the precopulatory movements), indicating the potential existence of a separate neuronal pathway for its activation. But this put the question how the stridulation is modulated by the brain control circuits to induce such additional courtship elements. This is the aim pursued in section 3.6. 


\subsubsection{Proctolin and female stridulation (Ch.b.)}

Stridulation in female Ch.b. could reliably be induced by injection of proctolin into the protocerebrum. The responses were robust and restricted to specific sites not obviously different from the effective sites in the males (Ch.b.). The stridulatory responses began 10-20 sec after the injection of proctolin and lasted for 5-10 sec. Only 1-2 sequences of $1^{\text {st }}$ order were performed (Fig. $\left.3-12, A_{1}-A_{3}\right)$, following a sin-

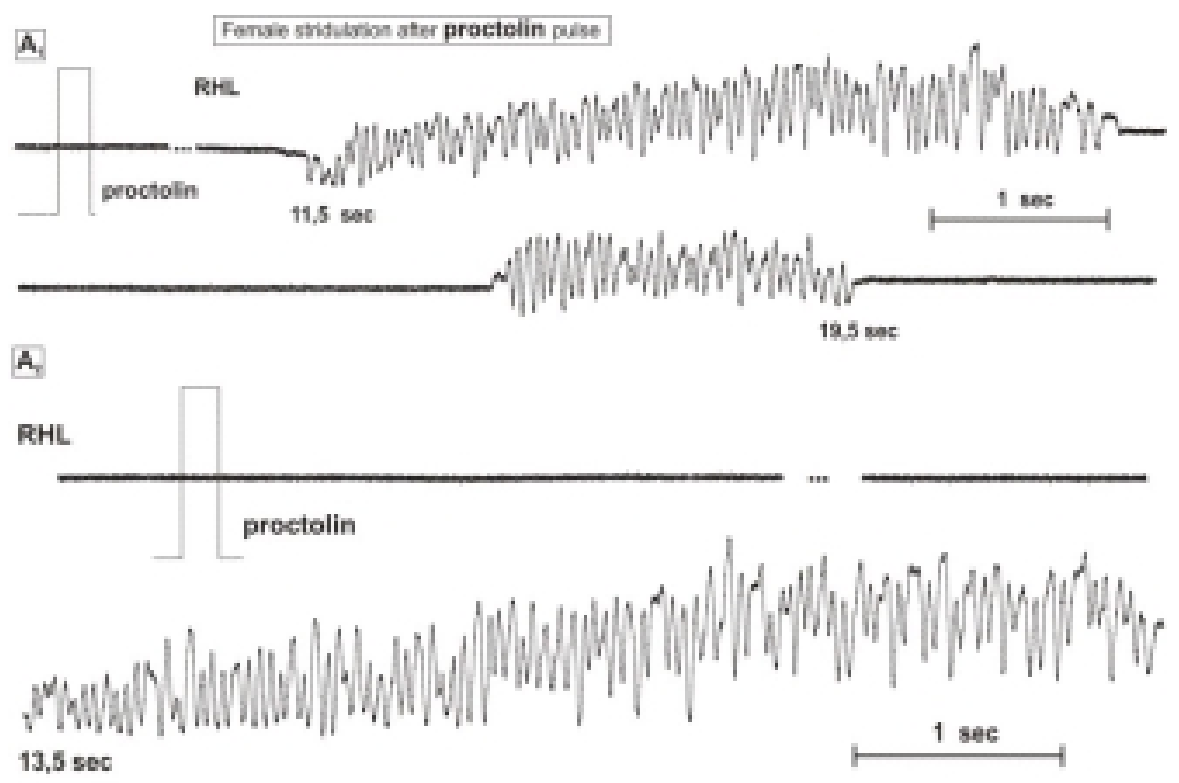

A.

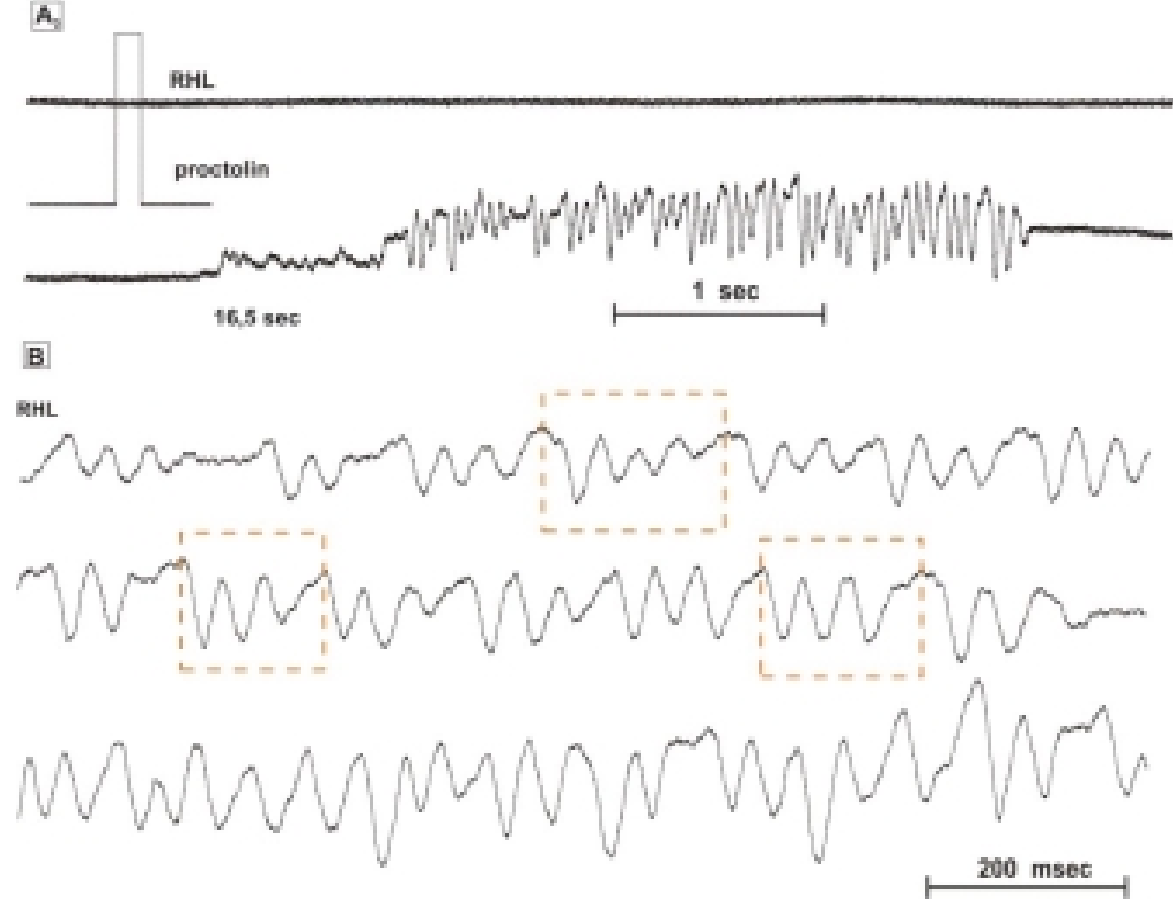

Fig. 3-12 Proctolin-initiated female stridulation (Ch.b.) $A_{1}-A_{3}$. Song sequences stimulated by single proctolin injection; $B$. The hindleg movements of the female song was less regular compared to that stimulated in males (red rectungles - single chirps). 
gle proctolin stimulation.

The stridulatory movements were not as precise as those performed by the males but the typical chirp structure (pulse/pauses) was available (Fig. 3-12, B). Females naturally stridulate with less accuracy compared to their con-specific males. The chirps lasted longer (200 msec), in comparison to $70-90 \mathrm{msec}$ of male songs. The "small stridulatory movements" of the male pattern were not distinguishable from the large ones in the females - the amplitude of all movements was almost the same.

Since proctolin reliably induced singing in female Ch.b. (in 4 of 5 animals tested) a new field of investigation could open up studying the neuronal mechanisms in the brain underlying the recognition of the con-specific song patterns (Lautschema) that is closely related to the control of singing responses performed by females in the courtship behavior. Previous studies with acoustic stimulation have clearly demonstrated that the female brain and not the methatoracic complex is responsible for the recognition of the highly specific singing pattern in Ch.b. (von Helversen, 1997). 


\subsubsection{Stages of proctolin-induced stridulation in Ch.b.}

Following the first proctolin stimulus applied to a particular site within the protocerebrum, the position of both hindlegs is gradually lifted from chirp to chirp

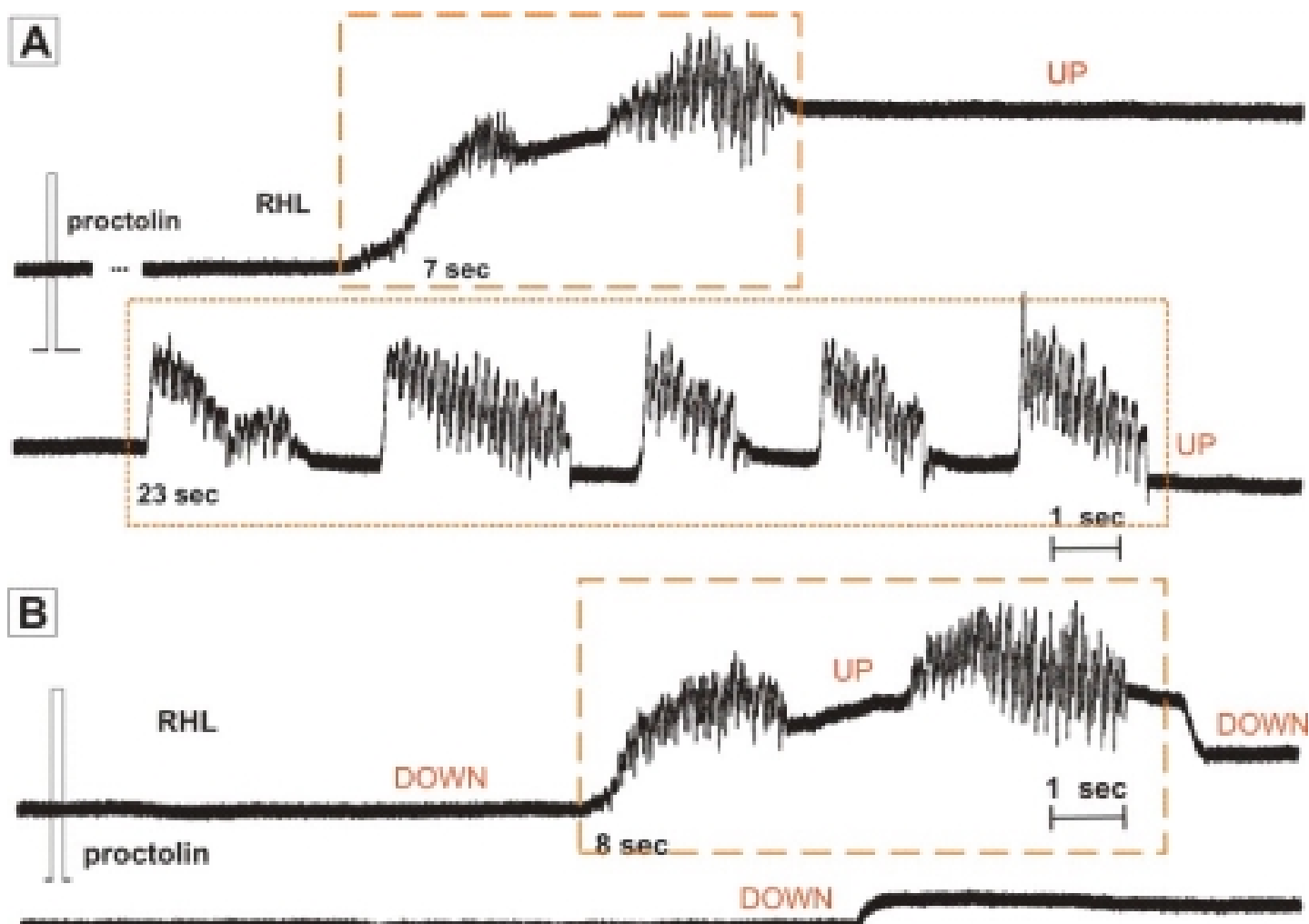

$23 \mathrm{sec}$

C
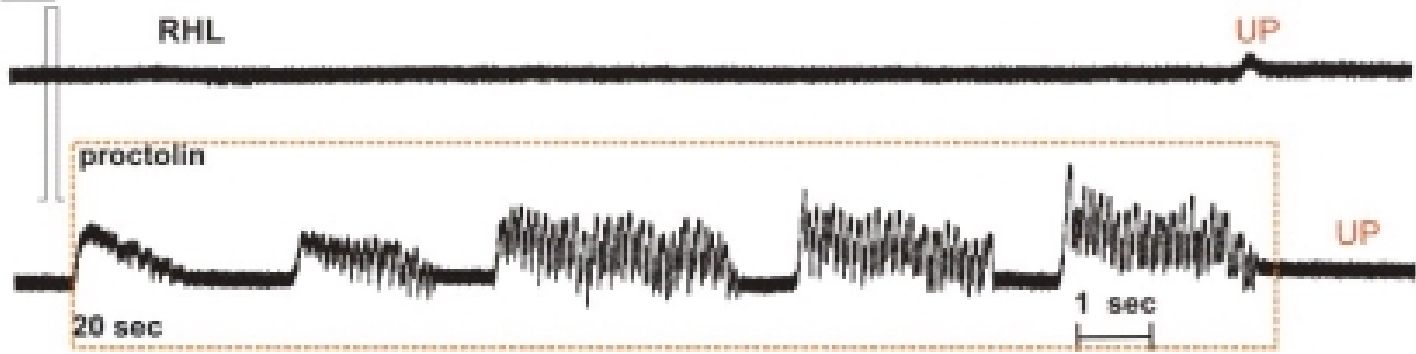

Fig. 3-13 Two different stages of proctolin-induced stridulation (male, Ch.b.). A. Initial stage consisting 2 long sequences, followed by maintaining stage containing 5 sequences; B. Only initial stage occurred; C. In some cases if the position of the hind legs is uplifted after stimulation only sustaining stage was performed

UP, DOWN - position of the hindlegs, along the responses

during the course of the first one or two sequences and kept in this elevated position for the remaining stridulatory activity elicited by this pulse (Fig. 3-13, A). This raised position (UP position) of the hind legs is the main characteristic of the high 
level of excitation, described previously in singing males under natural conditions (section 3.1). During the uplift the hind leg perform one-two longer sequences, which is called here initiation of stridulation or initial stage (phase) of stridulation. If the excitation was weak the hind legs took down and usually no consequent sequences were produced as it was after one proctolin stimulation in the experiment, shown in Fig. 3-13, B. If the excitation after proctolin stimulation was strong the upposition of hind legs was kept and consequent $1^{\text {st }}$ order sequences of shorter duration (Fig. 3-13, A, the second red rectungle) were performed similar to the natural singing (section 3.1). The following sequences after establishing the hind legs at the up position during one-two $1^{\text {st }}$ order sequences were named here maintaining of stridulation. Interestingly in this experiment (Fig. 3-13, C) was recorded performance of song sequences only in the maintaining stage without initial stage owing probably to the kept "up position" of the hind legs during the whole $3 \mathrm{~min}$ interval preceding this stimulation.

\subsubsection{Dose effects of proctolin on the stimulation of stridulation}

One method to investigate the dose-effect of a drug injected into the brain is to alter the duration of the injection pulse, as it has previously been done to characterize muscarine-induced stridulation (Wenzel, 2000; 2002). However, it has to be considered that the injected volume is not necessary linearly related to the duration of the pressure pulse and variations may result from a number of parameters, such as the air pressure applied, the size of the capillary tip and the viscosity and the thickness of the tissue. Before each experiment, the end of the electrode was broken under visual control to an approximate tip diameter of 10-15 $\mu \mathrm{m}$. Capillaries used in different experiments therefore had different opening diameters of their tips, through which the drugs could be ejected with differing ease. Variances in the tip diameter were compensated by setting the pressure and/or the pulse duration to values that ejected a small droplet into air, immediately before the capillary was inserted into the grasshopper brain. Breaking glass capillaries with two chambers with a forceps may have resulted in dissimilar openings of the chambers. Identical pressure pulses may have ejected slightly different volumes from 
both chambers leading to different dose-dependent effects and thus could be crucial for validity of the received data. In addition, higher proctolin doses injected may alter also the concentration changes of the proctolin diffuses troughout the tissue, competing to the clearance processes. Therefore, quantitative investigations were obligatory.

In series experiments after establishing a stable stridulation at typical stimulation parameters (sharp tip of the electrode, 40-50 psi air pressure, around 100 msec pulse duration, $\mathrm{C}_{\mathrm{M}}$ (proctolin) $=1 \mathrm{mM}$ ) the duration of the pulse was varied in progression manner - twice, triple, or $2 / 3,1 / 2,1 / 3$. Absolute values of the pulse duration (in msec), as it has been previously used (Wenzel, 2000. 2002), do not

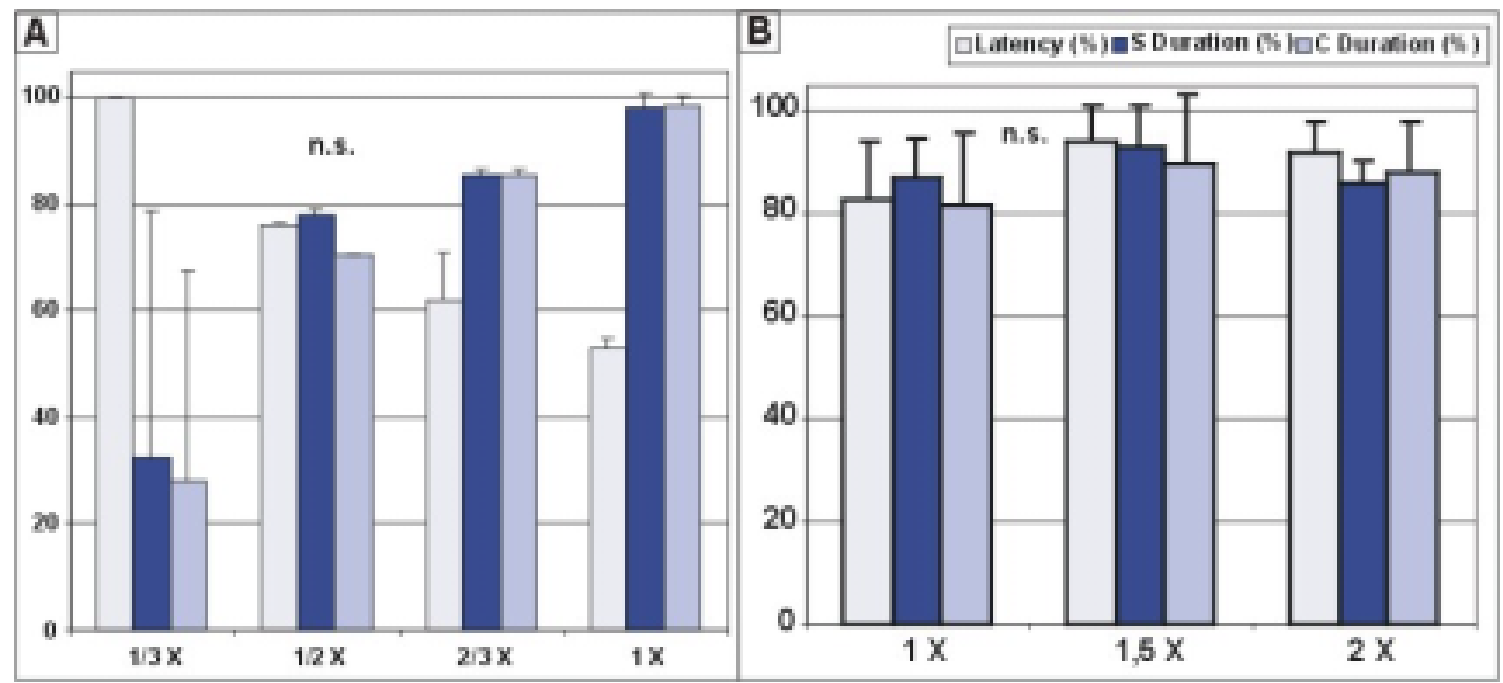

Fig. 3-14 Proctolin-induced stridulation (males Ch.b., $\mathrm{n}=5$ ) was slightly dependent on the dose of proctolin injected (using protocol 2-2-2 each stimulation parameters were repeated twice).

A. By shortening the duration of proctolin pulse injected the latency gradually increased and the durations slightly decreased. At a definite amount (pulse duration) the stridulation suddenly stopped $(1 / 3 X)$. At these stimulation parameters the response was either 0 or around $80 \%$.

B. By increase the duration of the pulse injected no changes in proctolin responses were observed.

correspond to the same amount injected substance in two different experiments. Thus the data from two experiments could not be compared. That's why it was used a multiplication factor (1X, $2 X, 3 X$ or $2 / 3 X, 1 / 2 X, 1 / 3 X)$ to a duration of a control pulse, through which a stable stridulation has been achieved before. The results are shown in Fig. 3-14. 
By shortening the pulse duration, using protocol 2-2-2, a decrease of the response duration of $\sim 20 \%$, was observed. At a definite volume of injected proctolin, the duration did not decrease further - being either $80 \%$ or none. At volumes of $1 / 3 \mathrm{X}$ from the control pulse duration the responses shown in Fig. 3-14, A are either around $80 \%$ or 0 , during even particular experiment and did not reliably elicit responses. Lower doses $(1 / 4 \mathrm{X}, 1 / 5 \mathrm{X})$ did not induced any responses (data not shown). That means that the minimal volume of proctolin released almost the maximal response $80 \%$ under these experimental conditions.

In another series of preparations, the amount of proctolin injected to a fixed site in the protocerebrum was altered by varying the number of pressure pulses used for ejection of proctolin from the capillary (Fig. 3-14, C). A multiplication of the pulses proctolin injected did influence neither latency nor duration of the produced singing. Moreover the duration decreased slightly with the increase of the amount showing a saturation of the proctolin action.

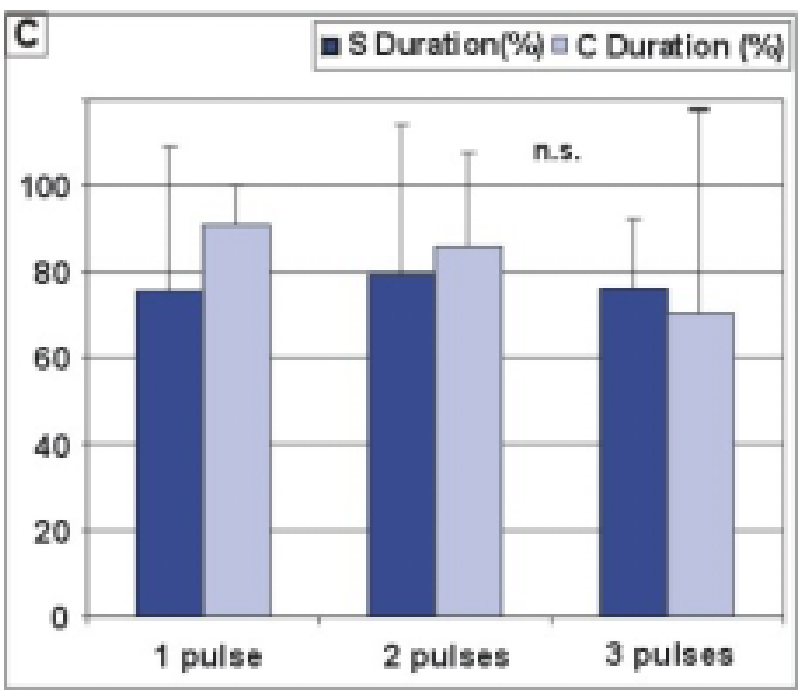

Fig. 3-14 C. Multiplication of the proctolin pulses injected (males, Ch.b., $\mathrm{n}=5$ ) Slightly decrease of the duration with the multiplication of the proctolin volume injected was observed. The SD increased with the increse of the volume.

In contrast to the duration, the latency between injection pulse and onset of stridulation was inversely related to the amounts of proctolin used for stimulation (Fig. 3-14, A). The mean relative latency increased gradually ( $50 \%)$ with shortening of the pulses to $1 / 3$ of the control pulse duration.

Proctolin dose/response (latency and duration) curve is shown in Fig. 3-15. The alteration of duration of proctolin responses of $\sim 20 \%$ with either decrease or increase of the volume of proctolin injected was observed. By the 1/3X pulse duration the "all or none" responses occurred (the red lines in the red rectangle) - either 
about $80 \%$ of the control response or none. The SD of $46 \%$ could serve as indication of "all/none" responses. Likewise with the increase of the dose the duration reached maximal values by $1,5 \mathrm{X}$ that corresponds to $120-150 \mathrm{msec}$ pulse duration used in most experiments. Further increase of the volume of the injected proctolin, $2 \mathrm{X}$ or $3 \mathrm{X}$ pulse duration, corresponding to $200-300 \mathrm{msec}$, the dose effect saturated and even inversed and the duration decreased (20\%). Interestingly by higher doses proctolin $3 X(\sim 300 \mathrm{msec})$ or 3 pulses in some stimulations "all/none" responses (either $100 \%$ or 0 ) were recorded, which can be represent by the meaning of $\mathrm{SD}=45 \%$.

Both duration and latency of proctolin-stimulated stridulation were dependent on the volume of the injected proctolin. While the alteration of the duration was in the range of $20 \%$, the latency showed $50 \%$ increase with the decrease of the dose. This may suggest that there is a limited number of proctolin receptors activated by the injected proctolin. Larger volumes of proctolin lead to a faster complete activation of available receptors but even injection of medium sized vol-

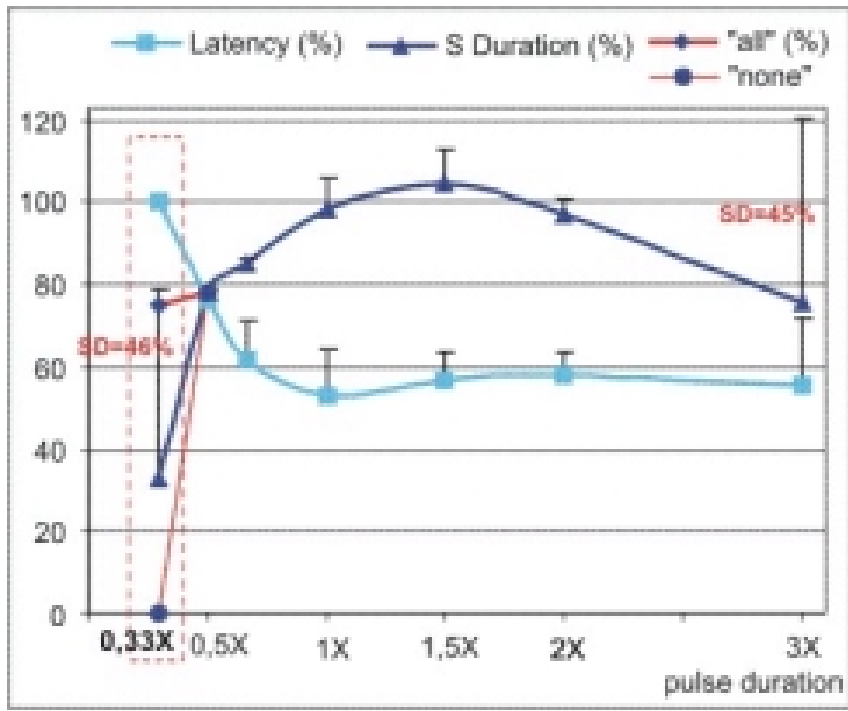

Fig. 3-15 Dose/response curves for latency and duration of proctolin-maintained stridulation (males, Ch.b.).

$1 \mathrm{X}$ pulse duration was $80-100 \mathrm{msec}$. The maximal response was reached by $1,5 \mathrm{X}$ pulse duration, which corresponded to 120-150 msec in most experiments. Thus the dose effect saturated and even inversed, by higher than $200 \mathrm{msec}$ pulse durations

umes are sufficient to activate the entire population of these receptors. 


\subsubsection{Temporal aspects of proctolin stimulation}

\subsubsection{Optimization of the fixed intervals in between proctolin pulses}

Repeated injections of proctolin, applied to the same site at regular intervals, induced stridulation of gradually increasing duration in the beginning. This increase in duration was associated with a decrease in the latency to the onset of singing and saturated after the third or fourth stimulation. From theron, proctolin stimulated stridulation of similar duration.

The intervals between individual proctolin stimuli were varied from 1 to $6 \mathrm{~min}$ in the sustaining phase without producing much of a change in the duration and latency of stimulated stridulation. After establishing the sustained phase through a series of stimulations, effects of previous injections of proctolin could be detected for up to $30 \mathrm{~min}$ in some preparations. Thus, proctolin-mediated excitation not only covers the period of the evoked stridulation $(5-60 \mathrm{sec})$ but may persist for up to 30 min in some grasshoppers.

Stable responses to proctolin stimuli were achieved by periodically injecting standard volumes of proctolin in fixed intervals (usually 3 or $4 \mathrm{~min}$ ) throughout the entire experiment.

Lengthening this interval caused alterations in latency and duration but shortening showed more complex responses, which will be presented in the next chapter. Fig. 3-16 shows series of five control experiments, using protocol 2-2-2. In a single preparation all 5 fixed intervals were

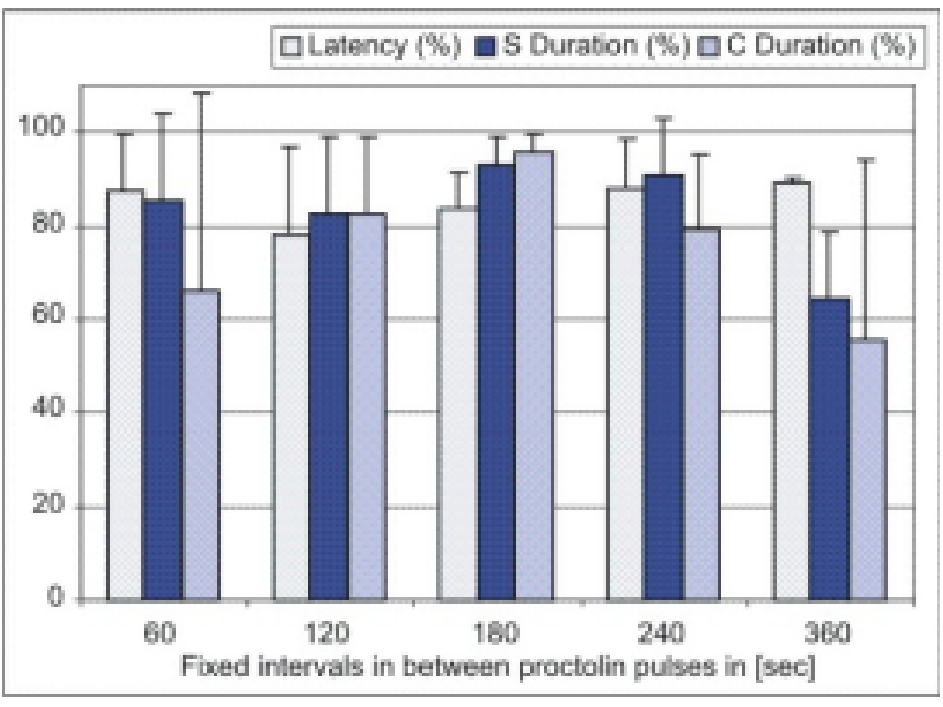

Fig. 3-16 Varying the fixed interval in between proctolin pulses (males Ch.b., $\mathrm{n}=5$ ), using protocol 2-2-2.

The SD is lower than $10 \%$ for all three stridulation parameters by fixed interval $180 \mathrm{sec}$.

twice tested. The al- 
terations of latencies and durations became larger with stimulus intervals shorter than 2 min or longer than $4 \mathrm{~min}$. This can be seen from the standard diviations (SD). With stimulus intervals of $3 \mathrm{~min}$, the SD of all three measured parameters was $<10 \%$. In this experiments again the relative values of $S$ duration and C Duration changed differentially with variations of the stimulus intervals. Therefore in all experiments shown further both parameters were measured and evaluated in all subsequent experiments.

3.2.5.2 Short inter-stimulus intervals decrease the stimulatory effect of proctolin on stridulation of Ch.b.

As it was shown above (Fig. 3-16) intervals in between proctolin pulses shorter than 2 min lead to irregular variations of durations and latency. Surprisingly, the duration of stimulated singing activity decreased with shorter intervals. This phenomenon was studied in a series of experiments, in which the interval between the proctolin stimuli was gradually shortened from $180 \mathrm{sec}$ down to $30 \mathrm{sec}$ and than lengthened up to $180 \mathrm{sec}$ (Fig. 3-17, A). The durations of the responses de-

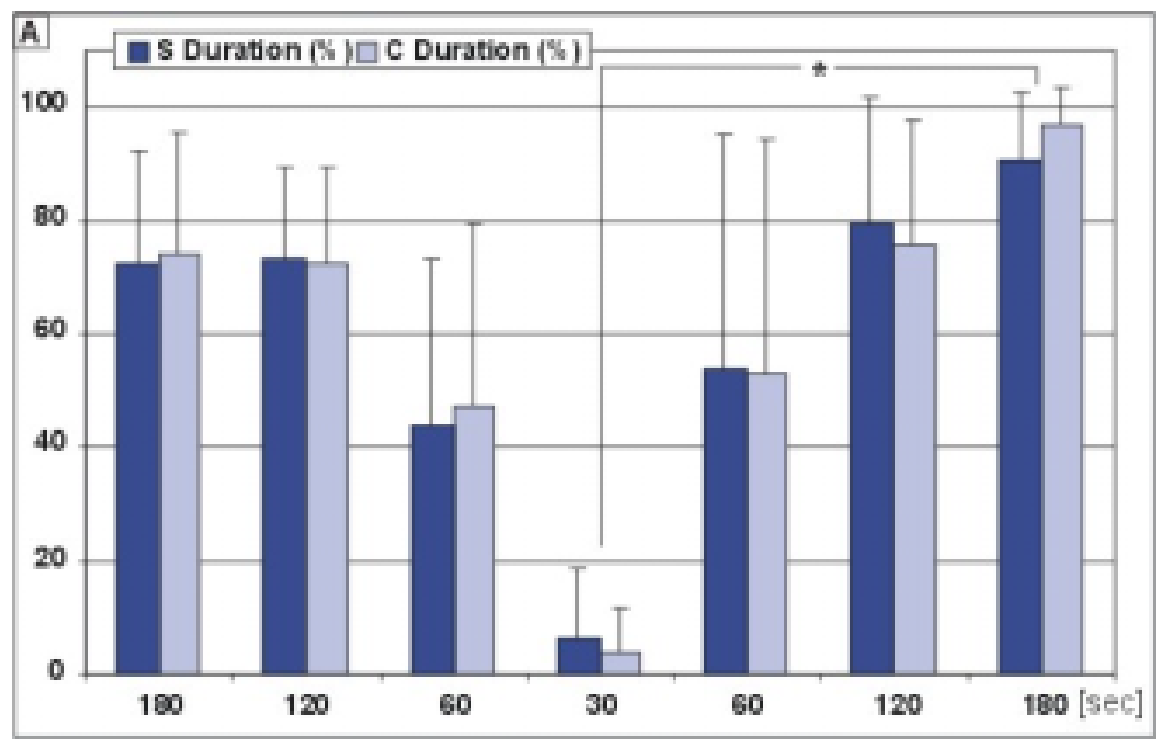

Fig. 3-17 After shortening the interval between proctolin pulses injected the duration of the singing (males Ch.b.) decreased $(n=4)$.

A. series of proctolin pulses with decreasing (from $180 \mathrm{sec}$ to $30 \mathrm{sec}$ ) and again increasing (from 30 up to $180 \mathrm{sec}$ ) intervals stimulated decreasing and again increasing durations of stridulation in Ch.b. that may indicate a mechanism of desensitization.

creased progressively when interstimulus intervals became shorter than $120 \mathrm{sec}$. With interstimulus intervals of $30 \mathrm{sec}$ only one stimulus at all successfully stimu- 
lated very short stridulatory movements. This result suggested a mechanism of desensitization, that reduced partly or completely the stimulatory effect of proctolin, depending on the duration of the interstimulus interval.

This experiment was repeated with a slightly different stimulation protocol (22-2). Each stimulus interval was once repeated, to separate its effect more clearly from influences of previous stimulations, applied at different intervals (Fig. 3-17, B)

Similar to the original experiment, stimulations at intervals of $60 \mathrm{sec}$ and even more pronounced at intervals of $30 \mathrm{sec}$ elicited stridulation of reduced and irregular durations. The results of both experiments shown in Fig. 3-17, A and B, demon-

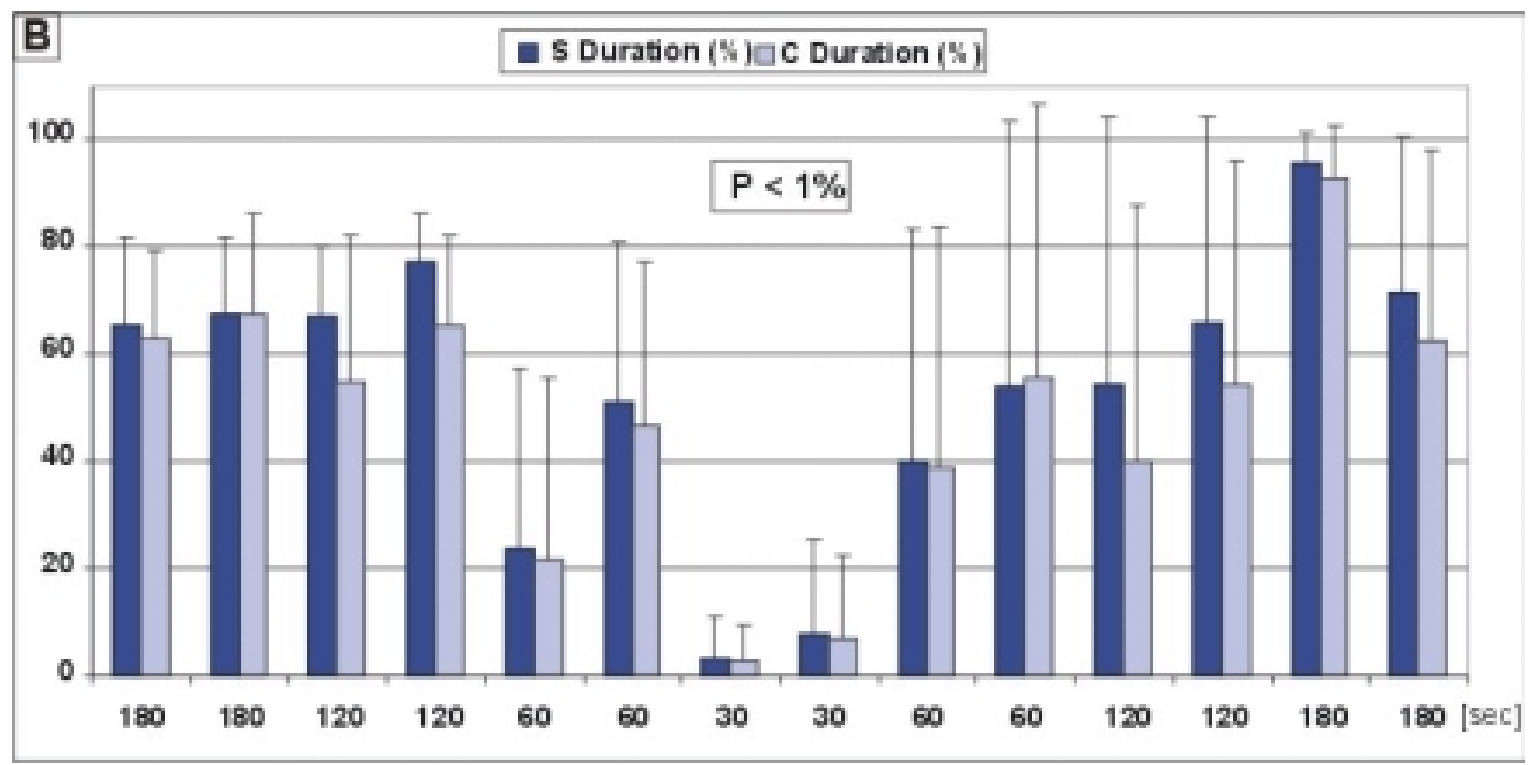

Fig. 3-17 B. Similar experiments as shown in part A but each interval was repeated once before changing to the next duration, using protocol 2-2-2 (males, Ch.b., $\mathrm{n}=5$ ).

strated desensitizing effects on the stimulatory action of proctolin when interstimulus interval were shorter than $120 \mathrm{sec}$. Interestingly, the stridulation after desensitization of proctolin mode of action through the shorter inter-stimulus intervals got shaky and the SD of the duration alterations arose to $50 \%$. In addition, after the desensitization phase by interstimulus interval of $180 \mathrm{sec}$ a longer singing was elicited than to the firsts stimuli in the protocol, but this over-response reversed to the initial values by the last injection of the protocol (Fig. 3-17, B). Compare to the slightly dose-dependence $( \pm 20 \%)$ of the durations of proctolin evoked singing, shown in the previous section. This suppression and/or over-excitation produced 
altering only the inter-stimulus interval may be an indication of time course dependent deduction and summation of ongoing proctolin signals within the neuronal circuit controlling the sound production in Ch.b.

\subsection{Methodological problems and adjusting the stimulation protocol to} the proctolin mode of action in Ch.b.

\subsubsection{Repeated stimulation with excitatory vs. inhibitory drugs (Protocol}

\section{3-1-4)}

Protocol 3-1-4 includes 7 or more proctolin injected pulses every 3 min and one injection pulse of the test substance between the $3^{\text {rd }}$ and the $4^{\text {th }}$ proctolin pulses. For that reason was called here Protocol 3-1-4. Fig. 3-18 summarizes all typical systematic errors, that have to be considered, when usinging this protocol and that may lead to miss-interpretations of experimental results. In a particular experiment, these errors (labeled A-E in Fig. 3-18) may or may not occur in different combinations.

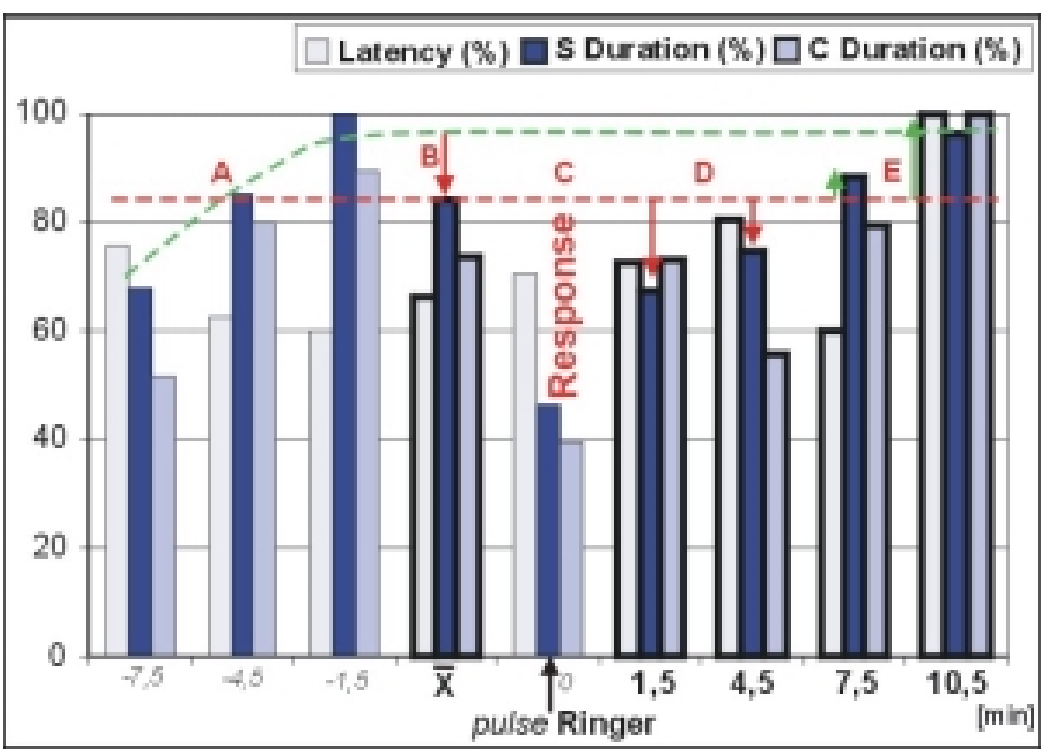

Fig. 3-18 Typical methodological problems, which may lead to artificial results.

A) Initial stimulation phenomenon - arousal of the excitation; B) The average of the proctolin stridulation in the initial stimulation stage could be shorter than in the maintaining stage; C) After injecting pulse Ringer or tested substance from the second chamber could be achieved stridulation; D) lowering the concentration of proctolin after pulse Ringer or tested substance (dilution effect); E) The average level of the response in the sustaining stridulation phase is higher than the average in the initial one $(B)$. 


\subsubsection{Initial stimulation phenomenon}

Repeated injections of proctolin, applied to the same site at regular intervals of $3 \mathrm{~min}$, induced stridulation of gradually increasing duration in the beginning. As illustrated by one typical experiment with Ch.b. (Fig. 3-18, A), this increase in duration saturated after the third or fourth stimulation and was associated with a decrease in the latency to the onset of singing. This phenomenon has been described also for muscarinic stimulation (Wenzel, 2000).

The effects result from accumulation of excitation that outlasts the interval between two consecutive stimulations. After this phase, the excitation (or its molecular correlate) generated by each stimulation pulse equals its degradation during period until the following stimulus, so that eych stimulation hits the circuit at the same pre-activated state. The phase corresponding to the arousal of the excitation in the beginning of the pharmacological stimulation (Fig. 3-18, A) without any changes of the pulse parameters (pressure, duration and size of the opening of the tip) is called here initial stimulation phase. None quantitative measurements and evaluations of this initial stage of the responses were possible. In most experiments the plateau or the stable level of excitation was reached after $2^{\text {nd }}$ to $4^{\text {th }}$ injected pulse. If the parameters (latency and duration) of the initial stridulation would be included in the evaluation the average (Fig. 3-18, B) values might be significant lower than those of the plateau.

Therefore, to assess effects on proctolin-stimulated stridulation, the test substances were applied only after the stable response phase was reached. This phase is called here sustaining stimulation phase (Fig. 3-18, the green line).

\subsubsection{Contamination of drugs in two-barrels microcapillary}

Two barrel injection capillary were used to inject two substances to exactly the same site within the protocerebrum. In some experiments injections of saline or a supposingly inhibitory substance stimulated stridulation (Fig. 3-18, C) although with significantly reduced duration. Since this was only seen after the excitatory substance (proctolin or muscarine) had previously ejected several times from the other chamber. The unspecific effect could be explained by the entrance of the proctolin into the other chamber of the injection capillary, due to the applied pres- 
sure or diffusion process. Injections of solution into a drop of vaseline had been shown that after repeated injection of a colored solution the following drops of the originally clear solution was vaguely colored. With repeated injections from the chamber containing the clear solution the contamination decreased (Wenzel, 2000). The amount of contamination seemed to be related to the size of the tip opening of the injection capillaries but quantifying control experiments had been not done.

The pressure-injected solution seems therefore to be pressed into the neighboring capillary chamber. Obviously it is found there in a lower concentration. This is in accordance with the observation that some substances are able to induce stridulation unexpectedly, like saline in some controls, but this was of significantly shorter duration than the previously proctolin-induced stridulation (Fig. 3-18, C).

For that reason to investigate the role of a given drug supposing to stimulate stridulation using two-barrel capillaries filled with known excitatory in the first chamber is not to be recommended. For investigations on the ability of a test substance to induce stridulation is advisable using one-barrel capillary.

On the other hand proctolin might be diluted in the first chamber after one or several pulses of the test substance. In experiments with potentially inhibitory substances a detected "inhibitory" effect could be due to a dilution of the afterwards injected proctolin solution (Fig. 3-18, D). This shortening of the stridulation due to a lower concentration of the activation substance could cause artificial significant "inhibititory effect" after evaluation with Friedman rank statistics if in 2-3 experiments from 10 takes place. The dilution effect could be potentiated if several pulses of the test substance are injected. Therefore could be misinterpreted as a dosis-dependent effect of the inhibitor.

Drastic effects could be expected, using this protocol, if the stimulation induced by the activating substance remains near the threshold for the initiation of stridulation and a small decrease of the concentration would completely suppress its performance. The "completely inhibition" reversed after several proctolin pulses, when the concentration reach the initial values. If once stopped the stridulation should been initiated again that means the controlling system could response after 
the $2^{\text {nd }}$ to $4^{\text {th }}$ pulses and latter is from the concentration independent (initiation stimulation phase). In all these cases every neutral substance could have an "inhibitory" effect. The main problem using protocol 3-1-4 is that the systematic errors overlap the supposing inhibitory effect of the test substance.

Wenzel (2000), observing the upper effects, has concluded the following: "But as the shortening of the muscarine induced song was not observed for all substances tested which did not by themselves cause stridulation, and as not all inhibitory experiments did show this effect, the effect of any potential dilution can be neglected".

Each experimental result should be questioned whether a specific action of a drug used in a particular experiment is the only way to interpret the results or the experimental design and the equipment used (double-barrel injection capillary) may bias the outcome.

On the other hand, if the injection from the second chamber of test substance in between repetitive proctolin pulses with fixed interval $3 \mathrm{~min}$, contents some proctolin transited from the first one, it will cause desensitization of the system. The 1-2 min inter-stimulus interval is in the range of desensitization effects (the previous section). Thus the lack of response or decrease of the durations of the stridulation induced by the next proctolin pulse might owe to this desensitization and not to pharmacological inhibitory effect of the test substance.

To prevent or minimize some of the potential artifacts mentioned above, using Protocol 3-1-4, and to deal with potential desensitization of the proctolin receptors an alternative protocol was established that is described in the following section.

\subsubsection{Alternative protocol, optimized for studies with proctolin (Protocol}

\section{3-3-3)}

Protocol 3-3-3 consists of 3 injection pulses of proctolin from the first chamber of the capillary followed by 3 injections of a combination of proctolin and a test substance from the second chamber, and again 3 pulses proctolin from the first chamber. The intervals between all pulses are the same $-3 \mathrm{~min}$. 
The suggested Protocol 3-3-3 has many advantages in comparison to Protocol 3-1-4. Firstly, the concentration of the proctolin in the second chamber is the same as in the first chamber. So the dilution of the excitatory substance, proctolin, is prevented and the stimulatory potential of proctolin remains constant during the whole experiment. The inter-stimulus interval between all injected pulses is constant. Thereby potential desensitization effects owing to the shorter inter-stimulus intervals, when the test substance injected, are excluded.

The dilution of the test substance could not be eliminated completely - after the first 3 pulses proctolin only the test drug could be diluted by parts of ejected proctolin. The changes in the concentration of the test substance are not crucial. On the contrary in a given experiment effects on the proctolin-maintained stridulation can be observed, depending on the concentration of the test substance. Therefore, additional 3 pulses only proctolin were included in the stimulation protocol not only as a control of proctolin action but as an observation of the degree of the transition between the chambers and the concentration-dependent effects of the test substance.

The concentration variation of the excitatory substance during the whole experiment was solved using Protocol 3-3-3 but the problem - if the variation of the drop volume injected from both chambers has an effect - arose. Such experiments were done (section 3.2.4). Alterations in the limits of $20 \%$ for the durations and up to $50 \%$ increase of the latency could owe to dose proctolin effect. If these alterations are higher it could be observed as pharmacological effect. Concerning the "all/none" responses received by smaller amount proctolin injected, the latter criteria should be not applied to the silence responses. In case of "completely inhibition" occurred along the experiment the entire stimulation protocol was repeated using $1,5 \mathrm{X}$ longer pulse duration than that in the previous protocol. If the silent responses occurred again, than only the result was included in further evaluations.

The use of protocol 3-3-3 in addition to the "dose effect criteria", prevents the pharmacological approach from the main system artifacts of the stimulation protocol 3-1-4, using two-barrel injection capillary. 
The new protocol can be used to test both, inhibitory and excitatory effects of a second substance on proctolin-stimulated stridulation. From another side, applying combination of proctolin and other substances may be used to explore mechanisms of co-transmission and modulation in the cephalic circuits for song control.

Improving the stimulation protocol optimized to the proctolin mode of stimulatory action in Ch.b., was the most important and crucial stage of the present study, before beginning to examine any effects of different transmitters and neuroactive substances on proctolin-induced stridulation. 


\subsection{Proctolin- and cholinergic brain neurons control the sound}

\section{production in Ch.b.}

As demonstrated clear above stimulation with proctolin alone is sufficient to initiate and maintain singing behavior in male Ch.b. The proctolin system may therefore be a third signaling pathway in the song control system, besides ACh, [acting on both nicotinic (nAChRs) and muscarinic receptors (mAChRs)], and picrotoxin (PTX). The molecular mechanisms of muscarinic excitation have been described with considerable details (Wenzel, 2000) whereas the mechanism of proctolin action remained unknown. The existence of several substances capable of eliciting stridulation gives rise to a lot of questions: Which of these substances are necessary and/or sufficient to initiate and/or sustain stridulation? How do the modes of action of muscarine, PTX and proctolin interact each other? Do these transmissions act through separate mechanisms, modulate each other or stand at the different steps of one continuous process? How is the activity of different signaling pathways integrated to produce stridulation in adequate behavioral situations? Which other intracellular signaling pathways mediate excitatory or inhibitory neuronal responses?

To approach answers to these questions proctolin and muscarine were compared with regard to their effective sites of stimulation and their potency to initiate and maintain stridulation in Ch.b.

\subsubsection{Proctolin- and muscarine-sensitive sites for the stimulation of stridulation in the brain of Ch.b.}

In almost all experiments carried out in the present work stable stridulation could be stimulated by injections of proctolin into the anterior and dorsal neuropil to the central complex and protocerebral bridge. In these two regions the capillary tip was positioned close to the dorsal surface of the brain. The sites where proctolin elicited specific responses were very restricted. If the electrode was moved several $\mu \mathrm{m}$ to the side or deeper into the brain stridulation could no longer be stimulated. 
In previous studies on the species Omocestus viridulus (O.v.), stimulation sites effective for muscarine have been labeled with dextrans of high molecular weight (Wenzel, 2000; Heirich et al., 2001). All sites were located either within the upper or lower division of the central body or a neuropil lying posterior and dorsal from the central body. This neuropil contains fibers of the stridulatory command neurons (Hedwig, 1994; Hedwig and Heinrich, 1997). Although a similar localization of stimulation sites in Ch.b. was not carried out, a huge number of stimulation experiments suggested the same brain regions to be effective stimulation sites to muscarine in this species.

Using two-barrel electrodes filled with proctolin and muscarine respectively brains of Ch.b. were scanned to determine areas, where proctolin and/or muscarine could stimulate stridulation. It turned out that the area where proctolin elicited stridulation was smaller than the region that included effective stimulation sites for muscarine (Fig. 3-19, A). Both proctolin and muscarine stimulated stridulation (Fig.

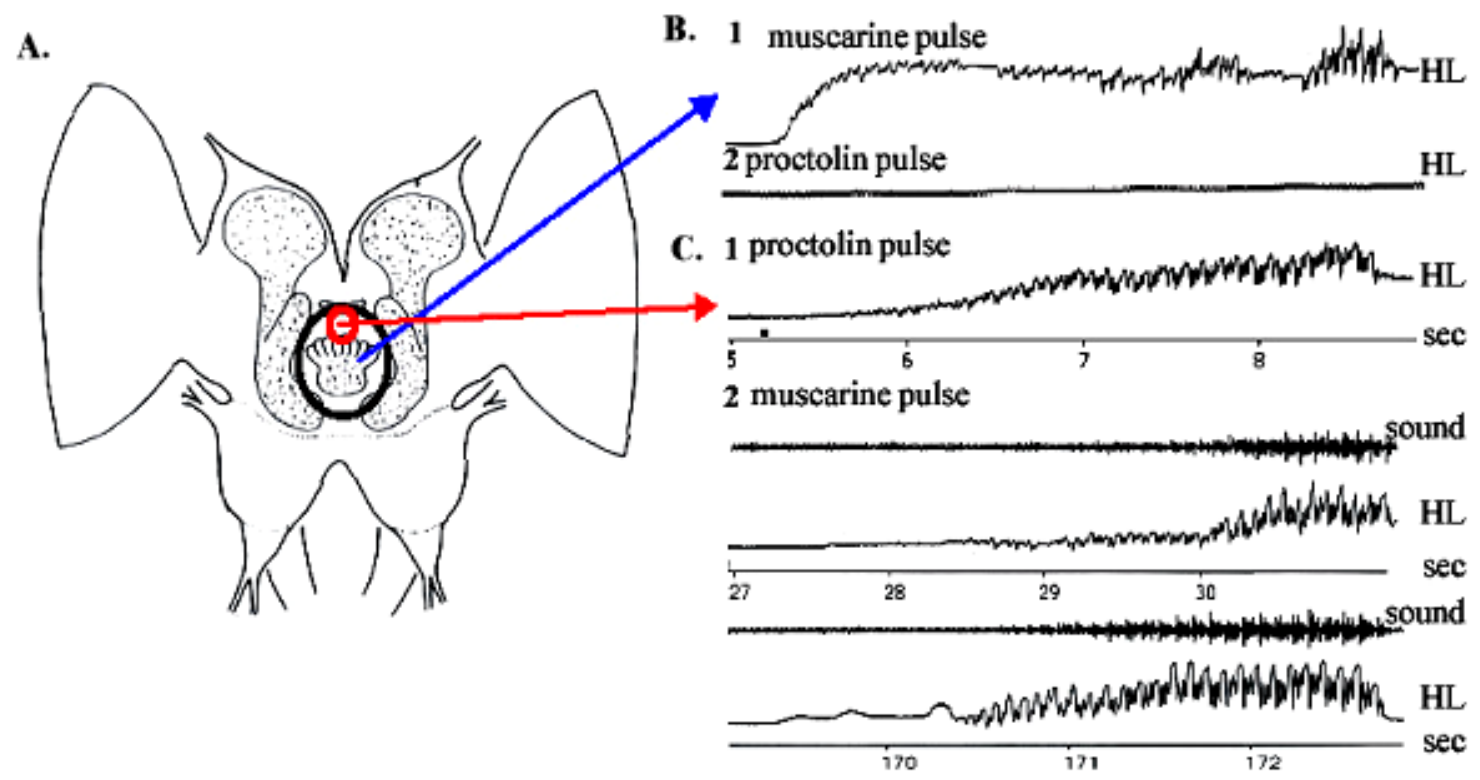

Fig. 3-19 Comparative view of proctolin- and muscarine - induced singing behavior (males Ch.b.).

A. Areas of the brain where it was possible to initiate stridulation through proctolin (in red) and muscarine (in blue);

B. Brain area, where a response was achieved to muscarine but not to proctolin (blue arrow, 1- response to muscarine; 2 - lack of response to proctolin pulse);

C. Brain area, mostly anterior portion of the central complex, protocerebral bridge and the neuropil dorsal to them, where both proctolin and muscarine stimulated stridulation (red arrow). In this restricted spots the responses to proctolin were in all cases of shorter latency and duration (1 - response to proctolin; 2 - response to muscarine pulse). 
$3-19, C)$ in a region that included the anterior portion of the central body and the area between the central body and protocerebral bridge. Muscarine, but not proctolin, was capable of releasing stridulation (Fig. 3-19, B) in a much larger area that additionally included the whole central body, $\beta$-lobes of the mushroom bodies and the neuropil areas dorsal to them. Not a single stimulation was found, where proctolin but not muscarine elicited singing behavior.

\subsubsection{Comparison of proctolin- and muscarine-stimulated stridulation}

At sites where both proctolin and muscarine stimulated stridulation the characteristics of the behavior were different. In all experiments proctolin stimulated short stridulation after a short latency. At the same injection spots muscarine induced longer stridulation after longer latency (Fig. 3-19, C; Fig. 3-20). These data indicate different mechanisms by which proctolinerg and muscarinic stimulation mediates excitation in the control circuits for stridulation. Whether PRs are colocalized with mAChRs on the same neurons could not be excluded but this is undistinguishable with this experimental approach.

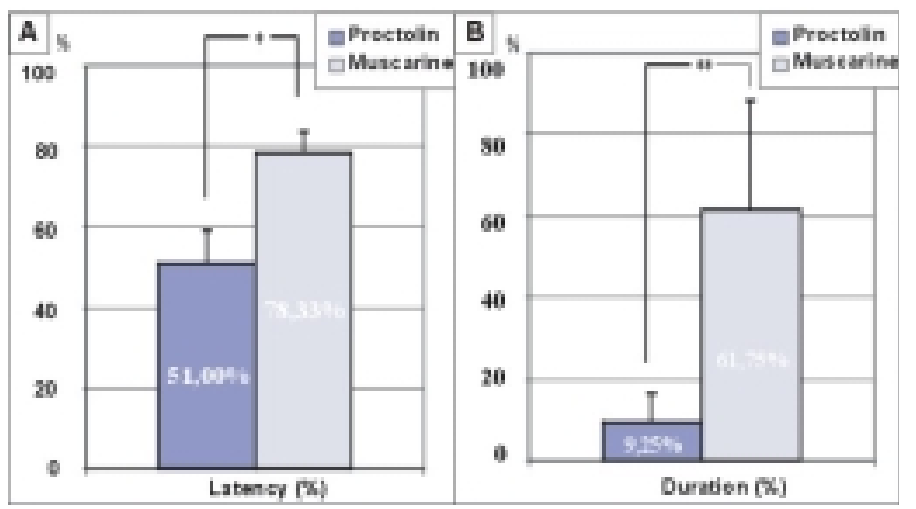

Fig. 3-20 Comparison between proctolin and muscarine-stimulated stridulation induced at the same spots in the protocerebrum of males Ch.b.

A. The latency of proctolin-induced stridulation was shorter $(P<0,05)$; $B$. The duration of the proctolin produced singing was much shorter than muscarinic $(P<0,01)$. 


\subsubsection{Combination of proctolin- and muscarine-induced stridulation}

In a series of experiments was tested, whether stridulation repeatedly stimulated by proctolin or muscarine was altered by an additional pulse of the other excitatory substance. By using protocol 3-1-4, with an inter-stimulus interval of 3 and $4 \mathrm{~min}$, the effects of both proctolin and muscarine on the induced stridulation were studied. A single pulse of muscarine injected in between two proctolin pulses increased the duration of the proctolin-induced stridulation (Fig. 3-22, A), but had no effect on the latency (Fig. 3-21, A). The additional stimulatory effect of muscarine lasted about $3 \mathrm{~min}$. On the other hand, proctolin injected in between two muscarinic pulses prolonged the muscarinic responses (Fig. 3-22, B) but shortened their latencies (Fig. 3-21, B). The effect of proctolin was longer and lasted longer approximately 6-10 min.

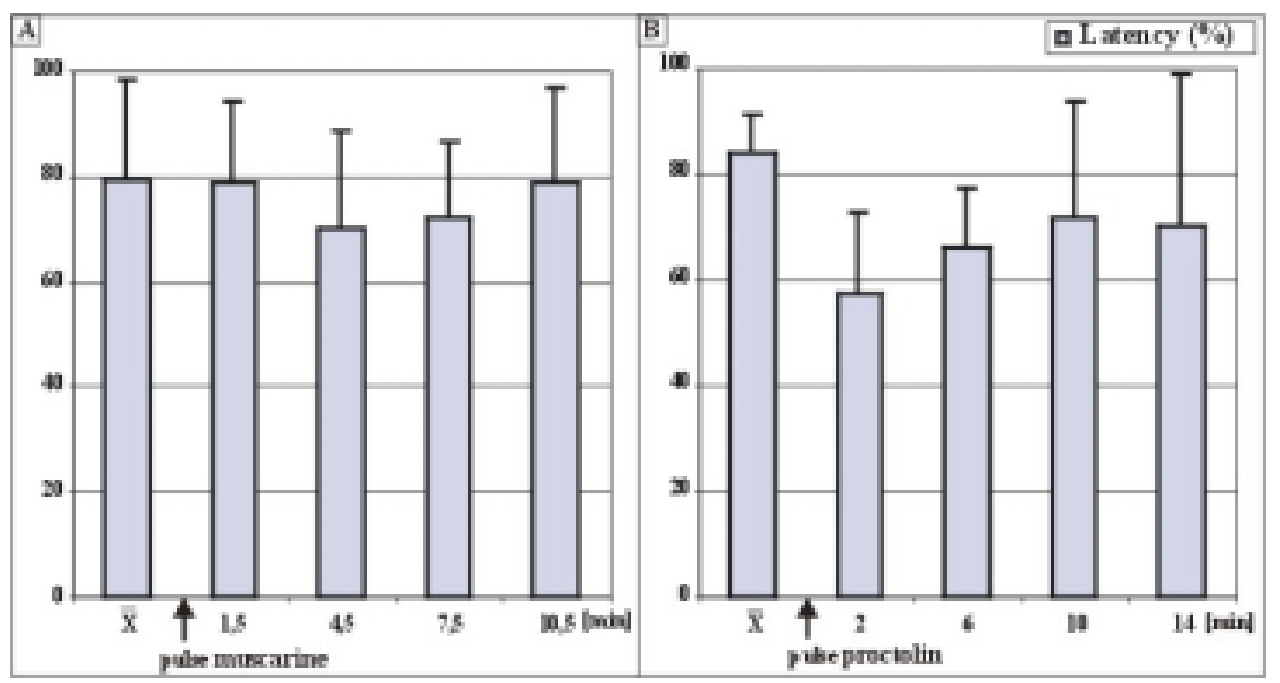

Fig. 3-21 Effects of additional proctolin and muscarine on the latency of the respectively induced stridulation (Ch.b)
A. No effect of muscarine on the latency of proctolin-stimulated stridulation ( $n=7, n . s$.
B. Shortening of the latency of muscarinic response by proctolin ( $n=4, n . s$. 

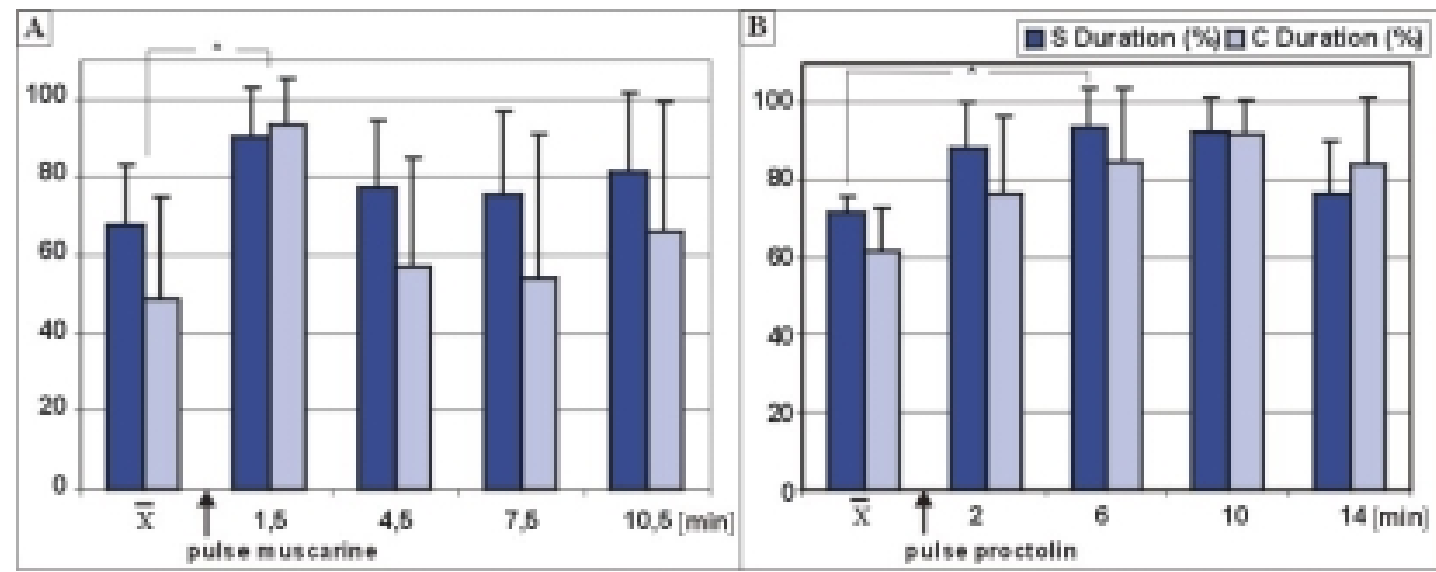

Fig. 3-22 The influence of both proctolin and muscarine on the relevant induced singing behavior (males, Ch.b.)

A. Muscarine injected in between proctolin pulses increased the durations of the proctolinic response $(n=7)$

B. Proctolin increased longlasting the duration of muscarine-induced stridulation $(n=4)$

\subsubsection{Proctolin injection during muscarine-induced stridulation}

Proctolin was applied during muscarine-induced stridulation, in order to see whether proctolin can modulate ongoing singing behavior. The duration increased due to appearance of additional two shorter sequences. The proctolin pulse was

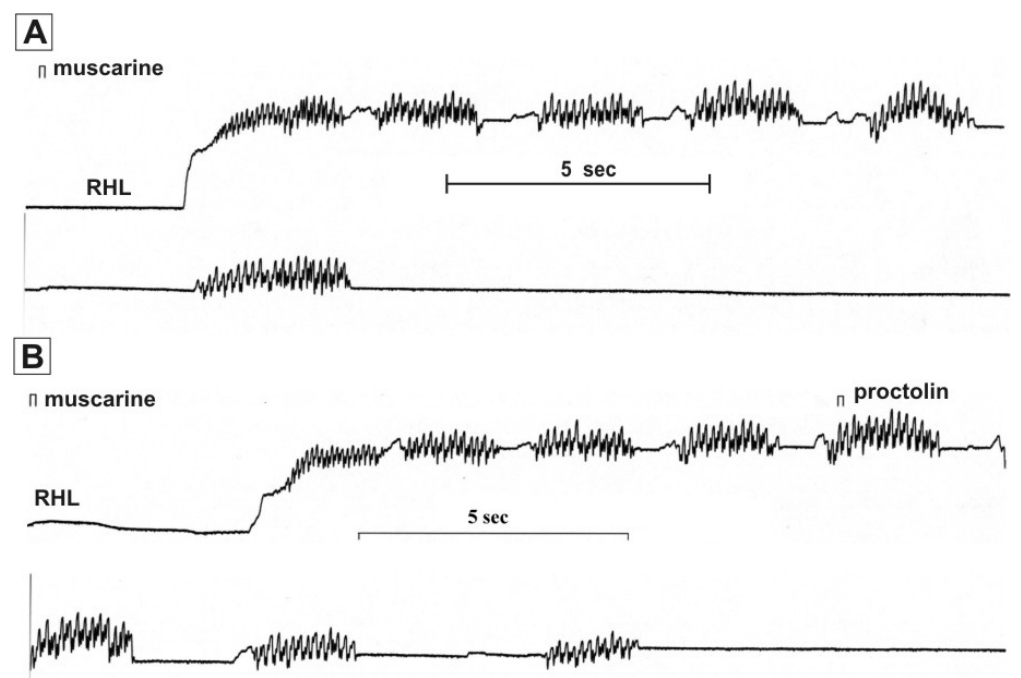

Fig. 3-23 The effect of proctolin injection, applied during a muscarine-induced stridulation in Ch.b.

A. Muscarine-induced stridulation

B. Proctolin pulse, applied $10 \mathrm{sec}$ after the onset of muscarine-induced stridulation leads to performance of two additional shorter sequences. 
applied $10 \mathrm{sec}$ after the onset muscarine-induced stridulation (Fig. 3-23, A, B).

Besides an increase of the duration, also some qualitative changes in the singing patterns induced by both substances were observed. Proctolin-stimulated song sequences were shorter $(1-2 \mathrm{sec})$ than muscarine-induced at the same brain spot (Fig. 3-24).

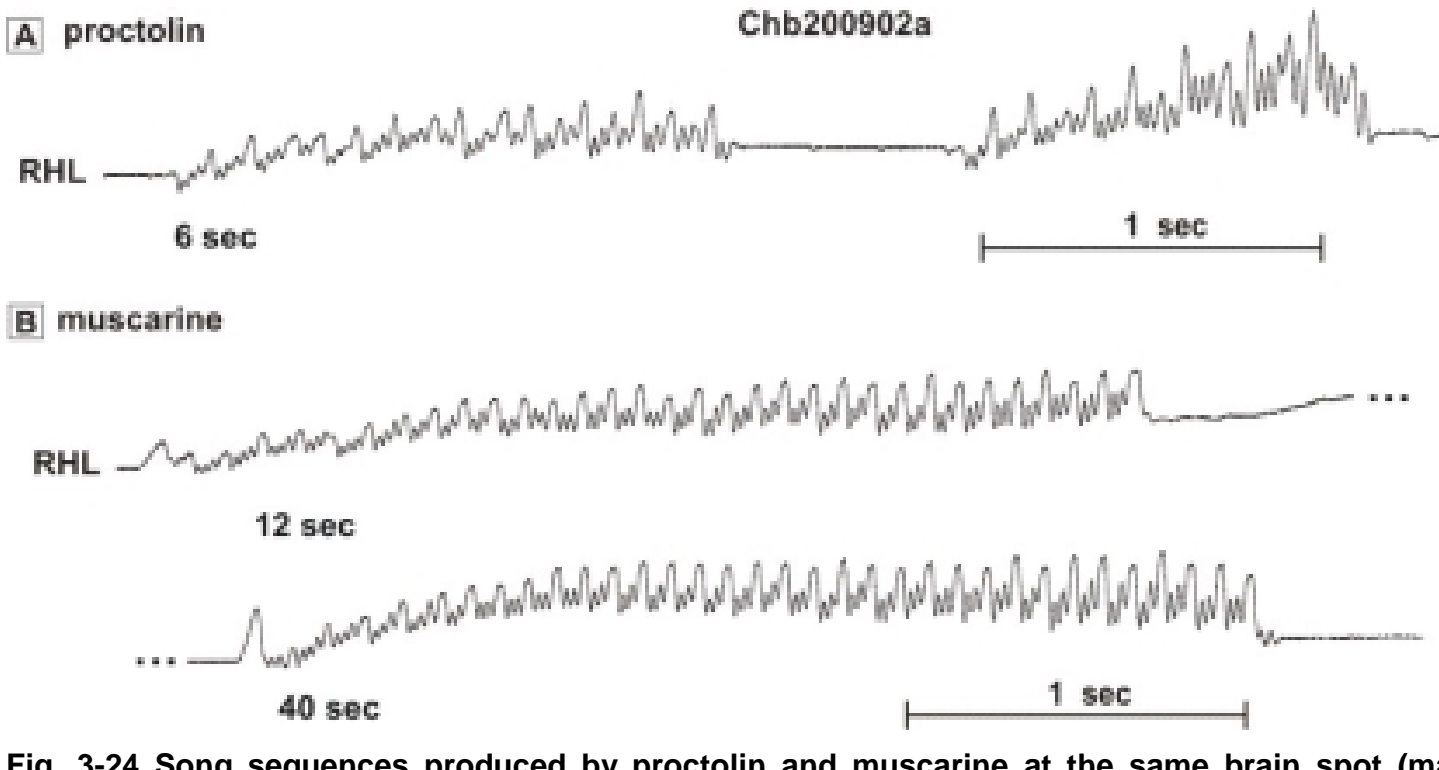

Fig. 3-24 Song sequences produced by proctolin and muscarine at the same brain spot (male,

Ch.b.) Proctolin-induced sequences $1^{\text {st }}$ order (A) were 1-2 sec shorter than muscarine-stimulated ones (B).

In two experiments proctolin modulated the muscarine-maintained singing inducing loud movements higher amplitude (Fig. 3-25, $\mathrm{B}_{1}$ ) and "single chirps" movements (Fig. 3-25, $B_{2}$ ) in between the other song sequences (Fig. 3-25, A).

Both proctolin and muscarine enhanced the effects of their counterparts leading to additional stridulation in Ch.b. males. It could not be excluded that both act on the relevant receptors localized on the same neurons, in the brain sites, where both stimulate stridulation and may modulate their responses. Coexpression of PRs and mAChRs has been reported in peripheral insect nervous system (Hinton et al., 1997). Alternatively, they may activate different neurons in the injection area, whose excitation converges on a common neural circuit that eventually stimulates stridulation. 
A muscarine before proctolin

Chb060902a

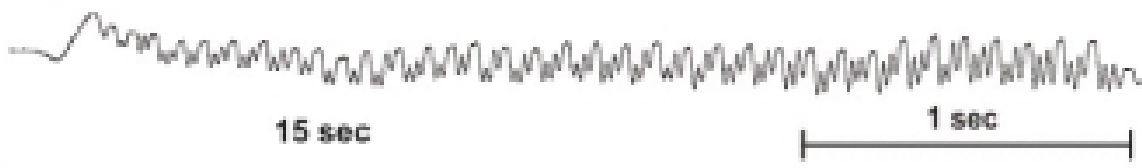

B, muscarine after proctolin

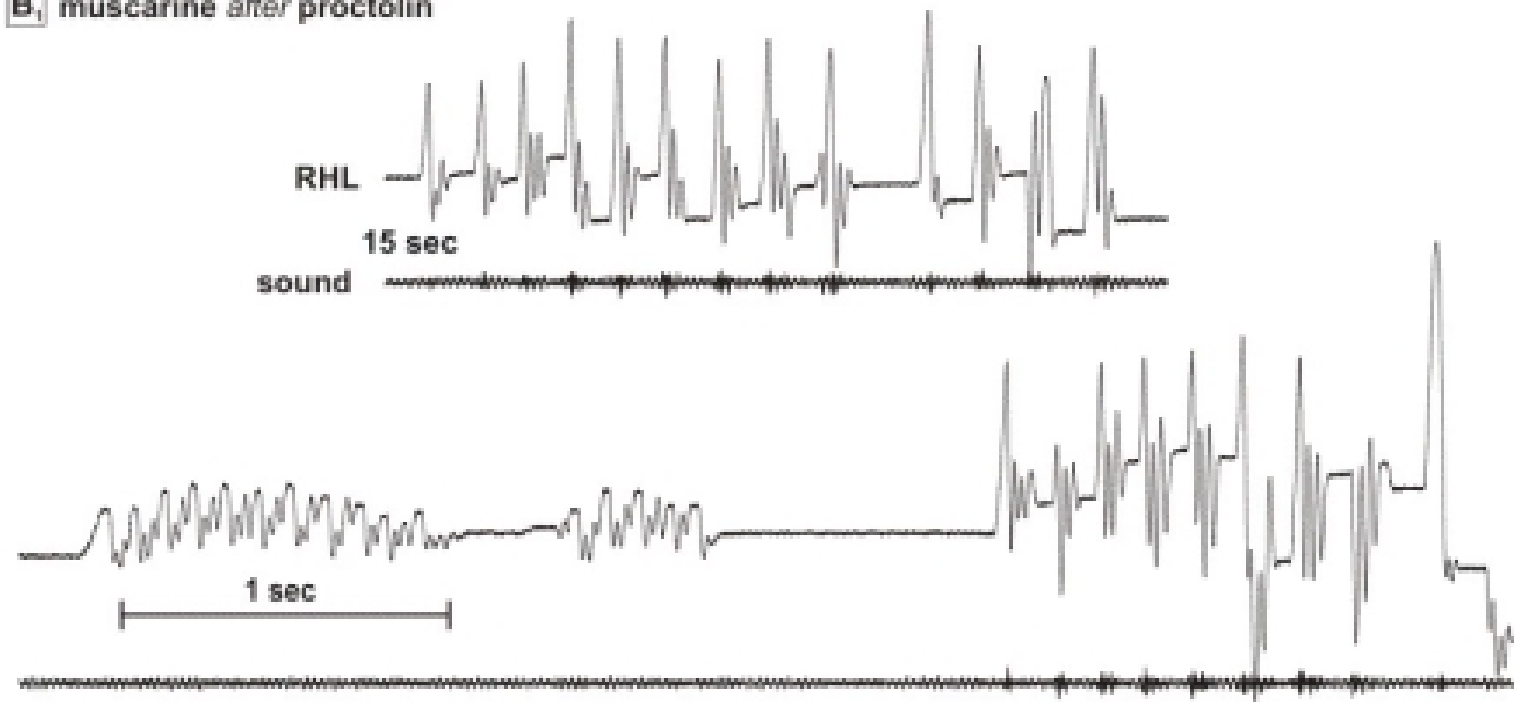

$\mathrm{B}_{2}$ muscarine after proctolin
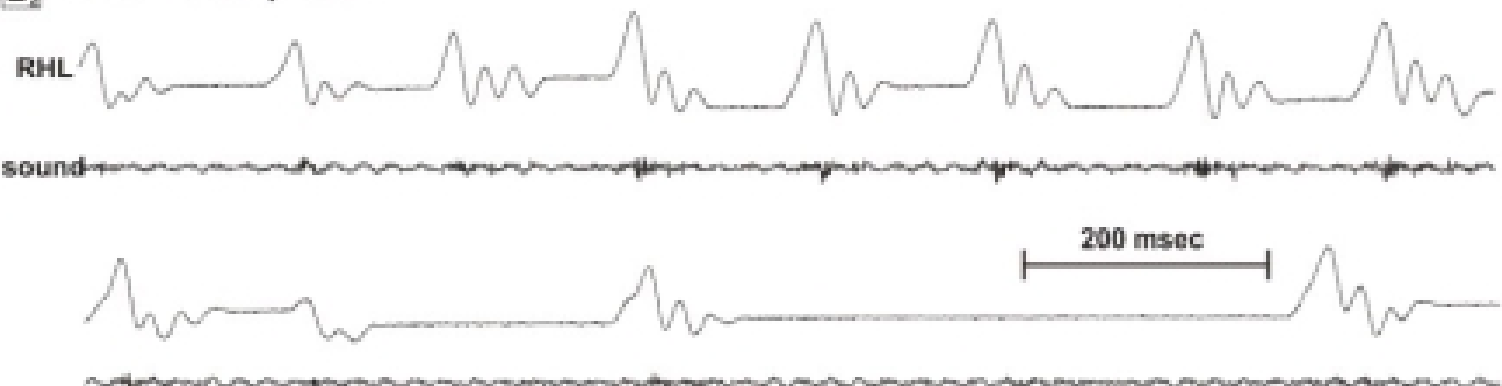

Fig 3-25 Modulation of muscarine-stimulated singing by proctolin (male, Ch.b.)

A. Typical muscarine-induced song sequence before proctolin application; $B_{1}$. Loud stridulatory-like movements with high amplitude; $B_{2}$. "Single chirps" uncoupled from each other. 


\subsubsection{Necessity of mAChRs for proctolin-induced stridulation}

In order to study the role of mAChRs activation on proctolin-induced and maintained stridulation they were blocked by the muscarinic antagonist scopolamine.

To prove the efficacy of the blockade of mAChRs involved in the control of singing behavior in Ch.b. by scopolamine, a series of control experiments was car-

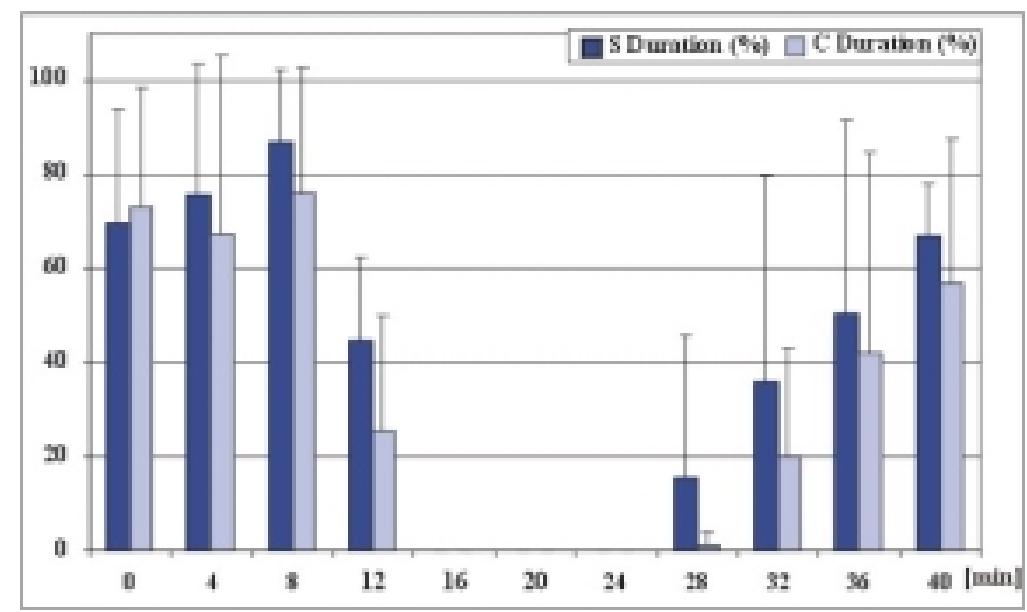

Fig. 3-26 Completely inhibition of muscarine-induced stridulation through mAChR blocker scopolamine (males, Ch.b., $\mathbf{n}=4$ ) (modification of protocol 3-3-3): 0,4,8 min - responses to muscarine; $12,16,20 \mathrm{~min}$ - responses to injections of muscarine and scopolamine $\left(C_{M}=1 \mathrm{mM} ; 1: 1\right)(P<0,01)$.

ried out. Repetitive muscarinic pulses from the first chamber of the capillary were followed by injection of muscarine and scopolamine $\left(C_{M}=1 \mathrm{mM} ; 1: 1\right)$ from the second chamber, using a modification of protocol 3-3-3. The protocol was modified in the following manner. Additional two pulses of muscarine were injected at the end of the protocol to completely reverse the effect of scopolamine and to reach the initial level of excitation before scopolamine application. The results are shown in Fig. 3-26. Scopolamine suppressed muscarine-stimulated stridulation completely in all experiments. The total inhibition lasted 16-20 min after which the responses restored gradually and reached the initial level at around 28-32 min after scopolamine application. Thus the effectiveness of scopolamine to block muscarineinduced singing in Ch.b. was very high, as it has been previously demonstrated in O.v. (Heinrich et al., 1997). This shows that scopolamine could be used as a potent antagonist of muscarine-induced singing behavior in Ch.b. The complete inhibition of singing through scopolamine was reversible but in a relative long period of time. 
The gradual increase in the durations of muscarine-stimulated stridulation after scopolamine-induced inhibition may result from both, a gradually disappearance of scopolamine from mAChRs in the stimulated brain area and the typical accumulation of muscarinic excitation that is also seen during the initial stimulation phase at a particular site.

To investigate the necessity of $\mathrm{mAChR}$ - activation for proctolin-induced stridulation a similar series of experiments was performed applying a combination of proctolin and scopolamine to the same sites, after stimulation of stridulation through proctolin. The results are shown in Fig. 3-27.

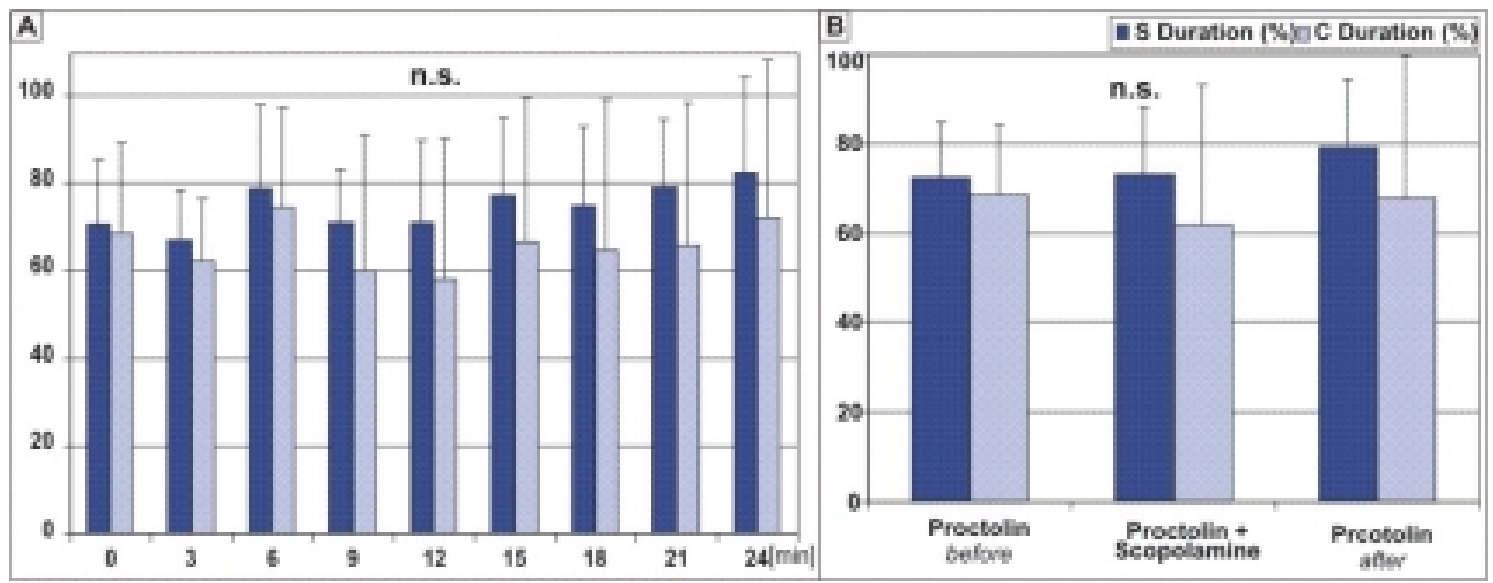

Fig. 3-27 Scopolamine, a potent mAChRs blocker, failed to alter proctolin-initiated stridulation (Ch.b., $\mathrm{n}=6$ ).

A. Responses (durations of stridulation) after repetitive proctolin injections (protocol 3-3-3): 0,3,6 min and 18,21,24 $\mathrm{min}$ - responses to proctolin pulses; 9,12,15 $\mathrm{min}$ - responses to combination of proctolin and scoplolamine $\left(\mathrm{C}_{\mathrm{M}}=1 \mathrm{mM} ; 1: 1\right)$

B. Average values of the durations of stridulation before, during and after scopolamine application. Slightly decrease of the $C$ Duration was observed but not of the S Duration (2 times higher SD).

Scopolamine did not affect the proctolin-initiated stridulation (Fig. 3-27, A). Only a slight (it was not significant and the $S D$ was 2 times higher than this of $S$ Duration) decrease of the $C$ Duration but not of $S$ Duration was observed. The higher SD by $C$ Duration than by $S$ Duration shows a higher grade of variation of the values during and after scopolamine application (Fig. 3-27, B). In other words the breaks (pauses) between sequences of $1^{\text {st }}$ order became shorter or disappeared, while the durations of the sequences were prolonged. This fact indicates that the pauses in between sequences of $1^{\text {st }}$ order might be also under regulation. 
The basal excitation is the same but the intrinsic properties of the brain network oscillatory activity is modulated.

\subsubsection{The effect of ACh on proctolin-induced stridulation}

ACh-initiated stridulation has been demonstrated to be blocked by dTC in O.v.. Also scopolamine has been shown to reduce the responses to ACh, injected each $15 \mathrm{sec}$, but has failed to block the initiation entirely. In other words, activation of mAChRs are not necessary for initiation stridulation produced by nAChRs activation through ACh in O.v.

In contrast to O.v., ACh stimulation, in all cases did neither initiate nor sustained stridulation under any stimulation conditions in Ch.b. (even through repetitive pulses at $15 \mathrm{sec}$ intervals). Moreover, the block of AChE through eserine did not produce any specific stridulatory movements. Thus combination of ACh and eserine has not mimicked muscarine action in Ch.b. (Hoffmann, 2003). There seems to exist differences between the control mechanisms of both most examined pharmacologically species - O.v. and Ch.b. Previously had been reported that in species whose basic patterns consist a rather simple up and down movements, for instance, O.v., O. ventralis and others (slow stridulatory movements, 7-8 Hz) ACh alone and nicotinic agonists reliably had elicited stridulatory movement patterns (Heinrich, 1997). In contrast to them, in Ch.b., Ch. molis, Stenobothrus rubicundus species with more complicated patterns and with fast stridulatory movements $(65-85 \mathrm{~Hz})$ ACh and nicotine in contrast to muscarine elicited only series of movements, which are not equivalent to the natural sequence pattern and usually included irregular hindleg movements (Heinrich, 1997).

Since most of the data on the nicotinic versus muscarinic portion of cholinergig excitation have been gathered in O.v. additional experiments were performed here for comparison. 
Applied alone ACh failed to induce any response even at the same spots, where proctolinstimulated stridulation (Fig. 3-28). Furthermore, ACh applied in combination with proctolin decreased the durations of proctolin-evoked singing (Fig, 3-29, A, B).

These results confirmed that ACh, when injected activates presumably nAChRs but not mAChRs,

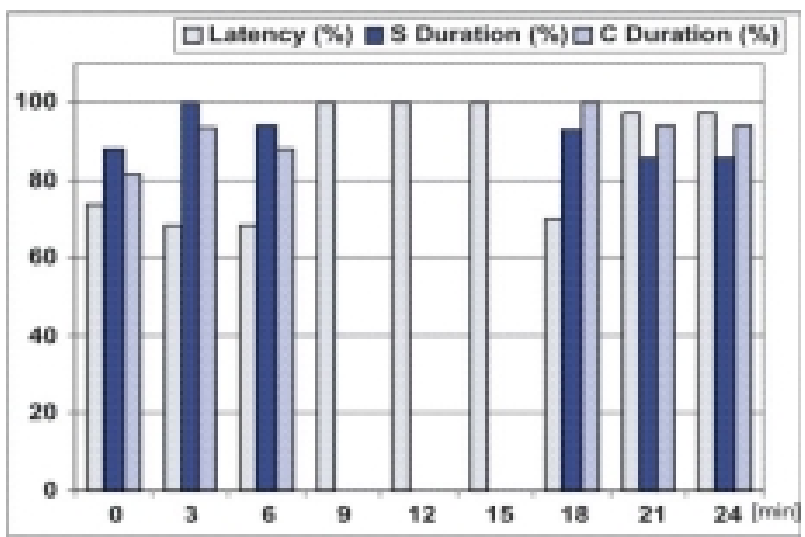

Fig. 3-28 ACh injected alone failed to initiate stridulation at the same sites in the brain, where proctolin reliably sustained stridulation (Ch.b., $\mathbf{n}=3$ ). 0,3,6 and 18,21,24 $\mathrm{min}$ - responses to proctolin; 9,12,15 min responses to $\mathrm{ACh}$ might owe to fast degradation by AChE activity. Therefore, one can propose that activation of nAChRs leads to triggering the performance of another behavioral program, which in turn opposes or excludes singing behavior (negative feedback). On the other hand, unbalanced pharmacological application of ACh could not mimic the fine synaptic processing and the fine regulated $\mathrm{ACh}$ release and fine tuned modulation of $\mathrm{nAChRs}$ on different arborisations of the neuronal circuit, which is presynaptic to the command neurons, which control the singing behavior.
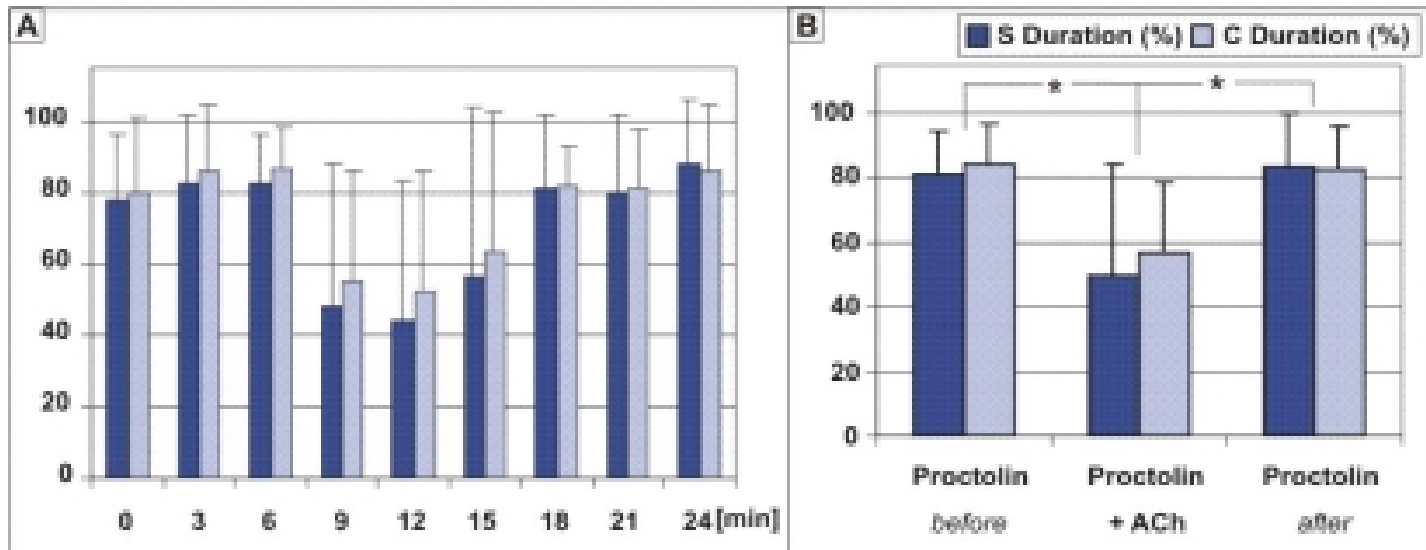

Fig. 3-29 The inhibitory effect of ACh on proctolin-maintained stridulation (Ch.b., $\mathrm{n}=6$ ).
A. Responses after repetitive injections proctolin (protocol 3-3-3): 0,3,6 and 18,21,24- re- sponses to proctolin pulses; 9,12,15 - responses to proctolin and $A C h\left(C_{M}=1 \mathrm{mM}, 1: 1\right)$.
B. Average values of the durations of proctolin-sustained stridulation before, during and after ACh application ( $\left.{ }^{*} \mathrm{P}<0,05\right)$ 


\section{Contribution of nAChRs to proctolin-induced stridulation}

The following experiments study the role of the interaction between proctolin and ACh transmission on the control of singing in Ch.b. (3.4.6.2.1) blockade of $\mathrm{nAChRs}$ during proctolin-initiated stridulation and (3.4.6.2.2) inhibition of AChE activity.

\subsubsection{Block of nAChRs through d-turbocurarine (dTC)}

ACh and nicotinic agonists had failed to induce any stridulatory movement in grasshoppers with complex and fast singing patterns of hindleg stridulatory movements (Heinrich, 2001). However, nothing is known whether the activation of $\mathrm{nAChRs}$ during muscarine-produced singing is necessary or plays any role.

dTC is known as a reversible competitive nicotinic receptor antagonist. It causes a drop in the opening frequency of the nAChR channels by reducing the number of receptor sites available for $\mathrm{ACh}$. The amplitude of the unitary current $\mathrm{i}_{\mathrm{ACh}}$ evoked in the presence or absence of dTC is identical (Hammond, 2001).

In 3 experiments dTC, applied together with muscarine, using protocol 3-3-3, increased the duration of muscarin-stimulated responses (Fig. 3-30, A). In contrast, the latency was slightly but not significant shortened (Fig. 3-30, B).

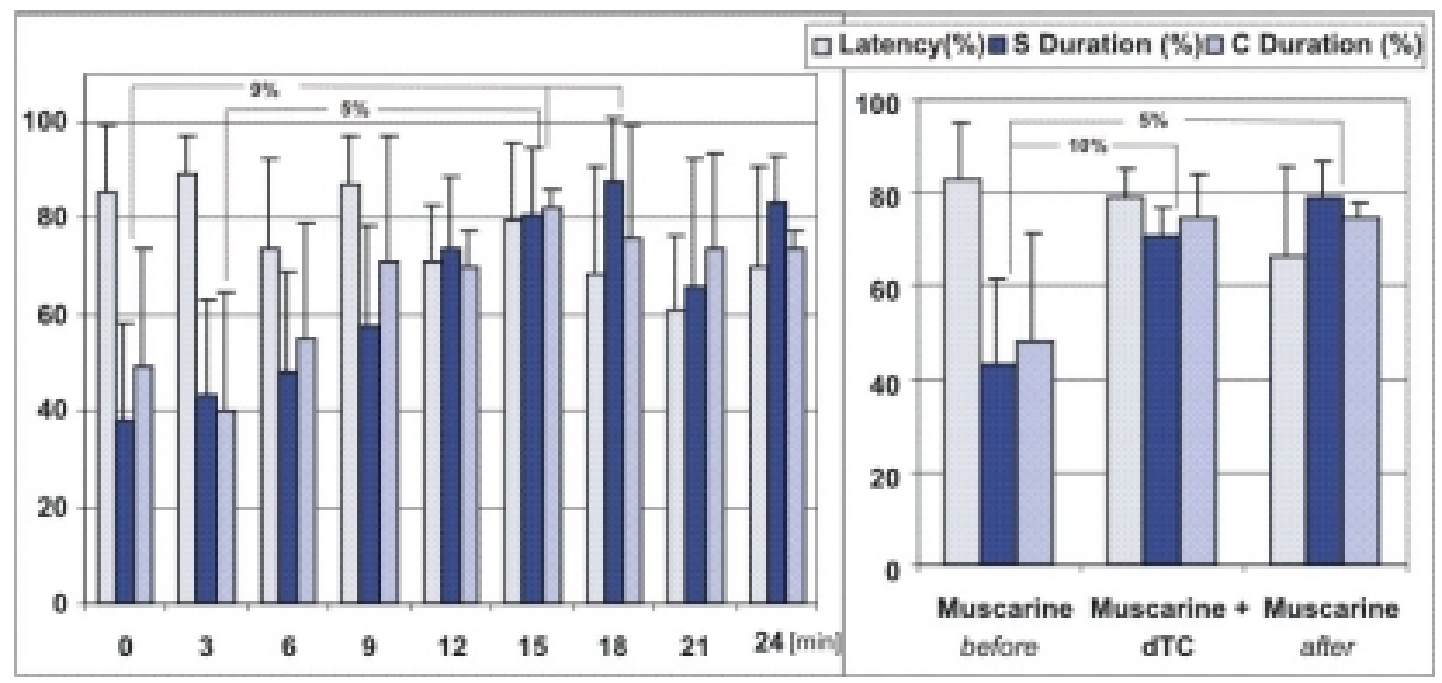

Fig. 3-30 Effect of dTC on muscarine-induced stridulation (Ch.b., $n=3$ ). A. protocol 3-3-3: 0,3,6 and 18,21,24 - responses to muscarine; 9,12,15 - responses to combination of muscarine and dTC; B. Average values (right): Latency $(p=0,09)$; $S$ Duration and C Duration $(p<0,05)$ 
dTC had similar effects on proctolin-stimulated stridulation in 3 experiments, namely a slight increase in the duration of singing (Fig. 3-31, A, B). The effect, most likely, results from effects on nAChRs in control circuits for other behaviors.

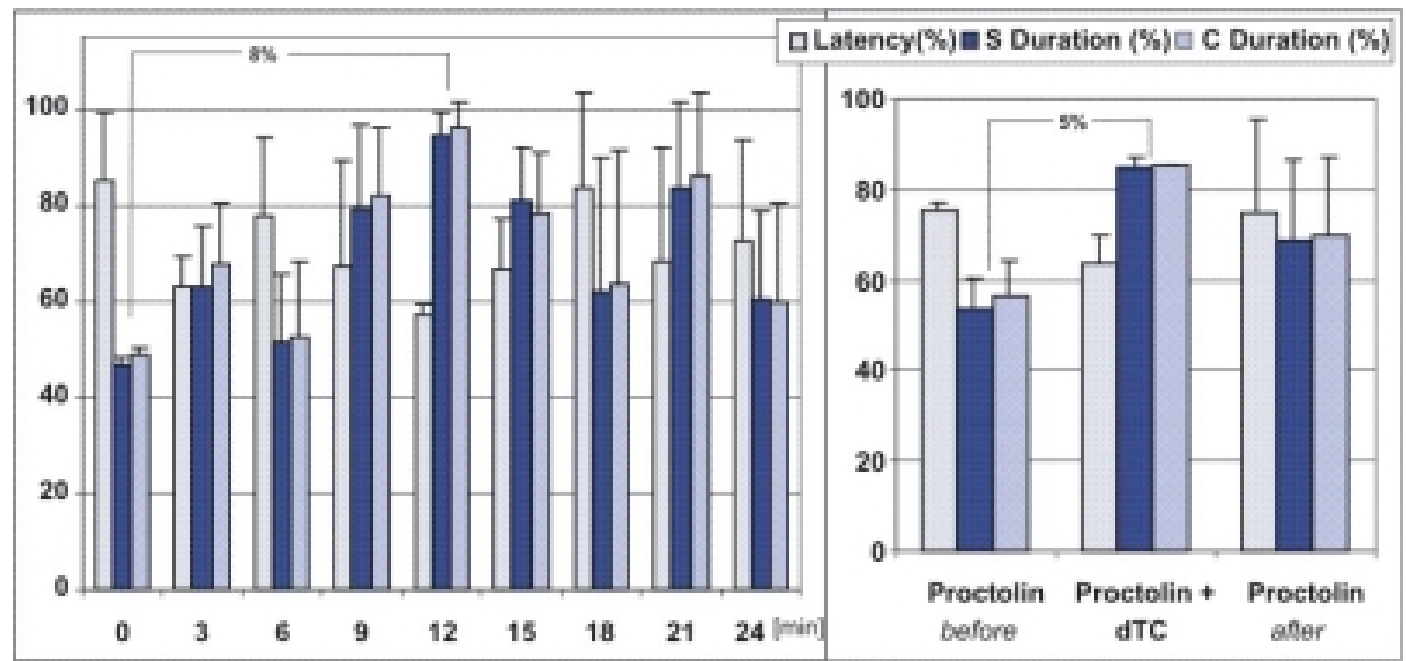

Fig. 3-31 Lengthening the durations of the proctolin-sustained stridulation through dTC (Ch.b., n=3). A. protocol 3-3-3: - 0,3,6 and 18,21,24 responses after proctolin pulses; 9,12,15responses after injection proctolin and $\mathrm{dTC}\left(\mathrm{C}_{\mathrm{M}}=1 \mathrm{mM}, 1: 1\right)$; $\mathrm{B}$. Average values of the responses before, during and after dTC application $(P<0,05)$.

In other 4 animals proctolin-induced stridulation was blocked partly or completely (in two of them) by application of proctolin and dTC (Fig. 3-32, A). The dTCmediated inhibition increases gradually over 3 applications of the proctolin/dTC mixture and was instantly reversed in the following proctolin application (Fig. 3-32,

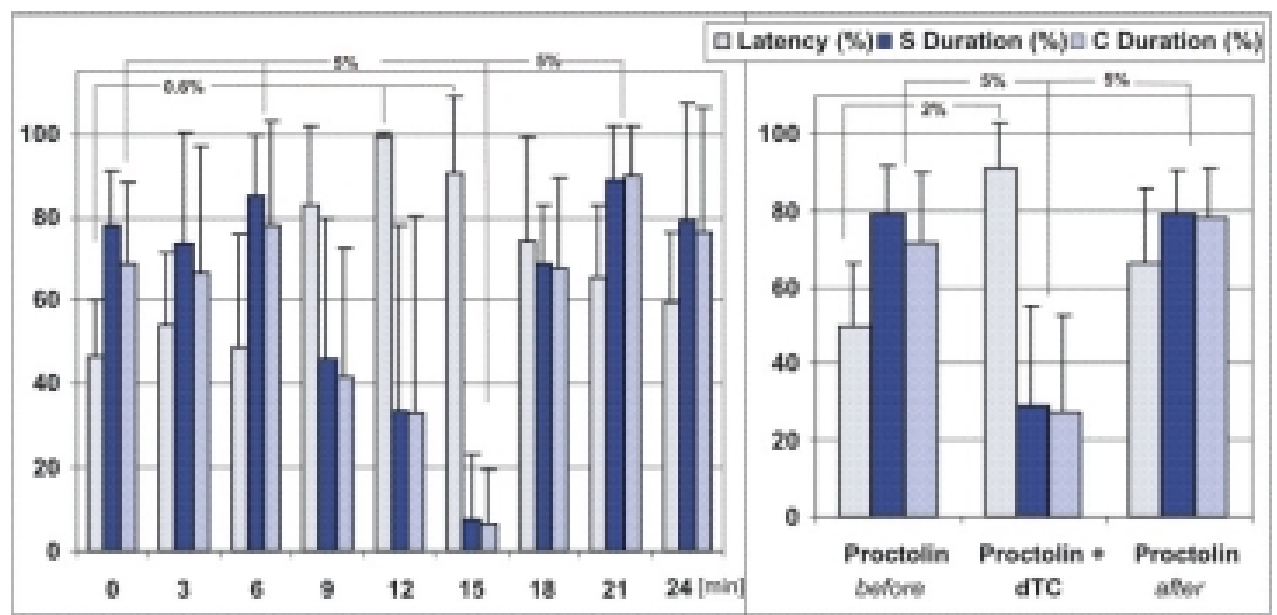

Fig. 3-32 Inhibitory effect of dTC on proctolin-stimulated singing (Ch.b., $n=4)$ A. protocol 3-3-3: 0,3,6 and 18,21,24 $\mathrm{min}$ - responses to proctolin; $9,12,15 \mathrm{~min}$ - responses to proctolin/dTC $\left(C_{M}=1 \mathrm{mM}\right.$, 1:1); B. Average values of the responses before, during and after dTC application $(P<0,05)$. 
A, B). In the same animals at a different stimulation site no such effect of dTC was observed.

\subsubsection{The role of AChE activity on proctolin-stimulated stridulation}

AChE activity is an object of a complex modulation accomplished by many neurotransmitters, neuromodulators and various neuroactive substances used in pharmacological investigations. The power and the character of the modulation depend strongly on the concentration of the substances. Opposite effects of particular substances on AChE activity at high and low concentrations have been described. Usually substances that alter the activity of AChE also influence the activity of nAChRs (open time, conductance, open probability nAChR, inactivation or desensitization). A well-known example for such complex effect on ACh transmission is eserine (physiostigmine, PHY), frequently used to enhance ACh signals by blocking AChE. Eserine is known as AChE inhibitor, but a direct agonistic action on nAChRs has been reported in both vertebrates (Schrattenholz et al., 1993; Schröder et al., 1994) and inveretebrates (van den Beukel et al., 1998; Jackson et al., 2002). On the other hand, it has been also reported that high concentrations of eserine block nAChRs (Pereira et al., 1993a, b; Schrattenholz et al., 1993; Schröder et al., 1994; Zheng et al., 1997). This leads to blockade of ACh transmission and ACh evoked responses. Furthermore, prolonged inhibition of AChE leads to desensitization of $\mathrm{nAChRs}$ and thus to short-term blockade of ACh transmission. $\mathrm{ACh}$, depending on its concentration, plays a modulatory function on the AChE activity being alosteric factor as it was reported in biochemical studies on its enzymatic action. In other words, the modulation of ACh release may affect indirectly the activity of both AChE and nAChRs. In many systems, mAChRs ( $\mathrm{M}_{2}$-like) are the main regulator of $A C h$ release. Furthermore, an unusual type $A C h R$ in insects, with both nicotinic and muscarinic features, had been described. Finally, as the fine modulation of nAChRs through neuropeptides comes in account, the complexity of this classical transmission goes far beyond our "classical" comprehensions.

In previous work, studying the effect of eserine on muscarine-stimulated stridulation, it has been reported that eserine at different concentrations had op- 
posing effects - from inhibition to enhancing the muscarinic responses in Ch.b. (Hoffmann, 2003).

Experiments examining the effect of eserine (eserinesalicilate, physostigmine, $\mathrm{PHY}$ ) on both muscarine and proctolin-initiated stridulation were carried out, using protocol 3-3-3. Eserine, co-injected to muscarine and proctolin affected in a complex manner both muscarine and proctolin-sustaining stridulations. Inhibition of both muscarine- and proctolin-stimulated stridulation were recorded in response to eserine. But the following proctolin and muscarine stimuli elicited singing longer than $600 \mathrm{sec}(\sim 100$ times the duration of initial proctolin injection).

These two experiments are presented in detail. Table 3-1 shows the latency and duration of the proctolin-stimulated stridulation before, during and after eserine co-injections.

Table 3-1

\begin{tabular}{|c|c|c|c|c|}
\hline Time (min) & $\begin{array}{c}\text { Protocol 3-3-3 } \\
\text { (No of the pulse): }\end{array}$ & Injection of: & Latency (in sec) & $\begin{array}{c}\text { S Duration (in } \\
\text { sec) }\end{array}$ \\
\hline $\mathbf{0}$ & $\mathbf{1}^{\text {st }}$ & Proctolin & $\mathbf{5 , 1}$ & $\mathbf{4 , 1}$ \\
\hline $\mathbf{3}$ & $\mathbf{2}^{\text {nd }}$ & Proctolin & $\mathbf{5 , 2 7}$ & $\mathbf{4 , 9 6}$ \\
\hline 6 & $\mathbf{3}^{\text {rd }}$ & Proctolin & $\mathbf{5 , 0 5}$ & $\mathbf{4 , 1 8}$ \\
\hline $\mathbf{9}$ & $\mathbf{1}^{\text {st }}$ & Proctolin + eserine & $\mathbf{0}$ & $\mathbf{0}$ \\
\hline 12 & $\mathbf{2}^{\text {nd }}$ & Proctolin + eserine & $\mathbf{7 2}$ & $\mathbf{5}$ \\
\hline 15 & $\mathbf{3}^{\text {rd }}$ & Proctolin + eserine & 51 & 5,5 \\
\hline 18 & $\mathbf{1}^{\text {st }}$ & Proctolin & 20 & $>600$ \\
\hline
\end{tabular}

Proctolin-stimulated stridulation before eserine application is shown in Fig. 3$33, A_{1}$. In response to the $1^{\text {st }}$ injection proctolin $(1 \mathrm{mM})$ and eserine $(0,5 \mathrm{mM})$ only active locomotor activity occurred. To the $2^{\text {nd }}$ and $3^{\text {rd }}$ pulse proctolin and eserine (Fig. 3-33, $B_{1}$ and $B_{2}$ ) specific stridulatory sequences were accompanied with unspecific movements, consisting stridulatory components. 


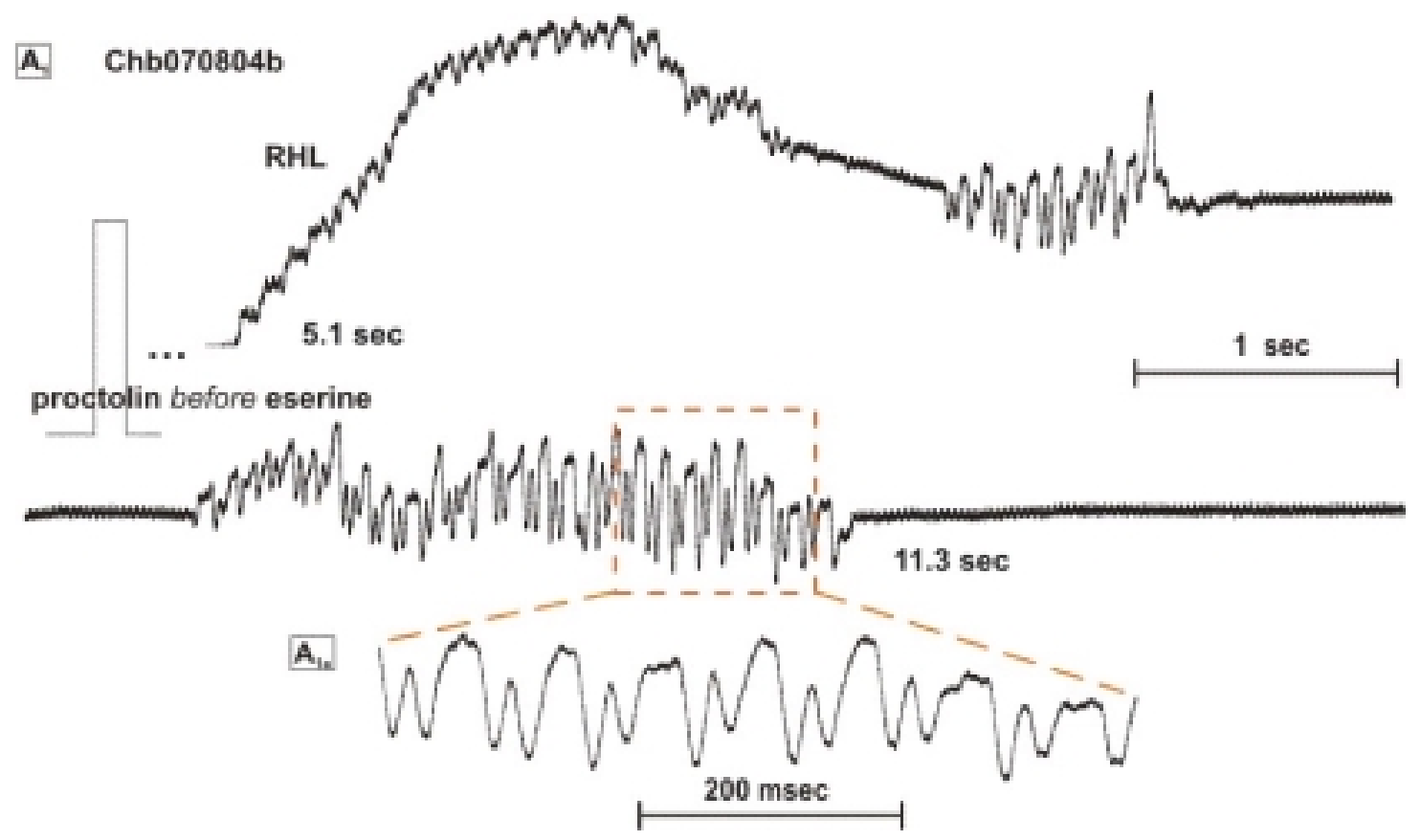

Fig. 3-33 $A_{1}$. Proctolin-initiated stridulation before application of eserine (male, Ch.b.).

$A_{1 a}$. The internal structure of proctolin-induced stridulation (chirps containing 4 syllables).

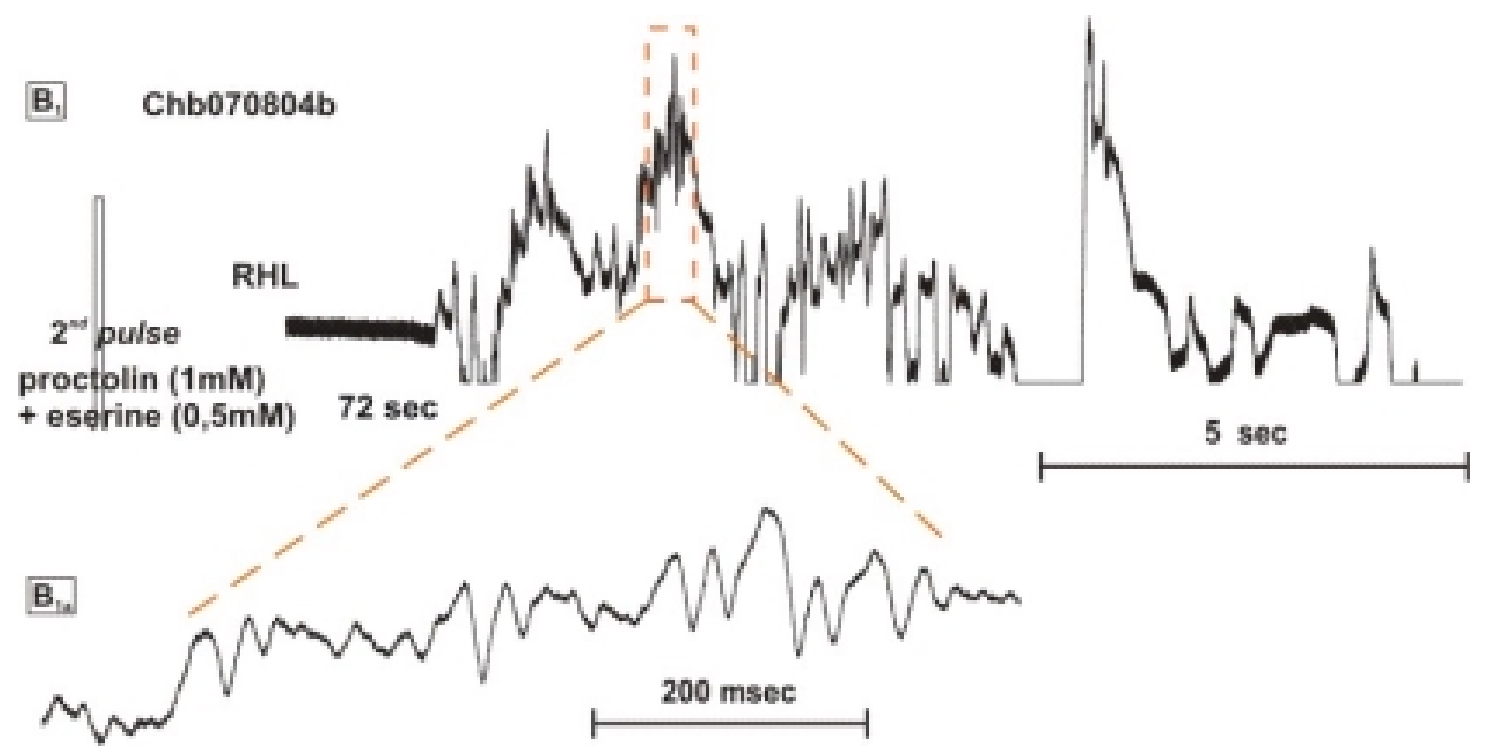

Fig. 3-33 $B_{1}$. Irregular stridulatory movements in response to the second pulse proctolin (1mM) and eserine $(0,5 \mathrm{mM})$. $B_{1 a}$. Single up- and down stridulatory movements accompanied with unspecific movements. 


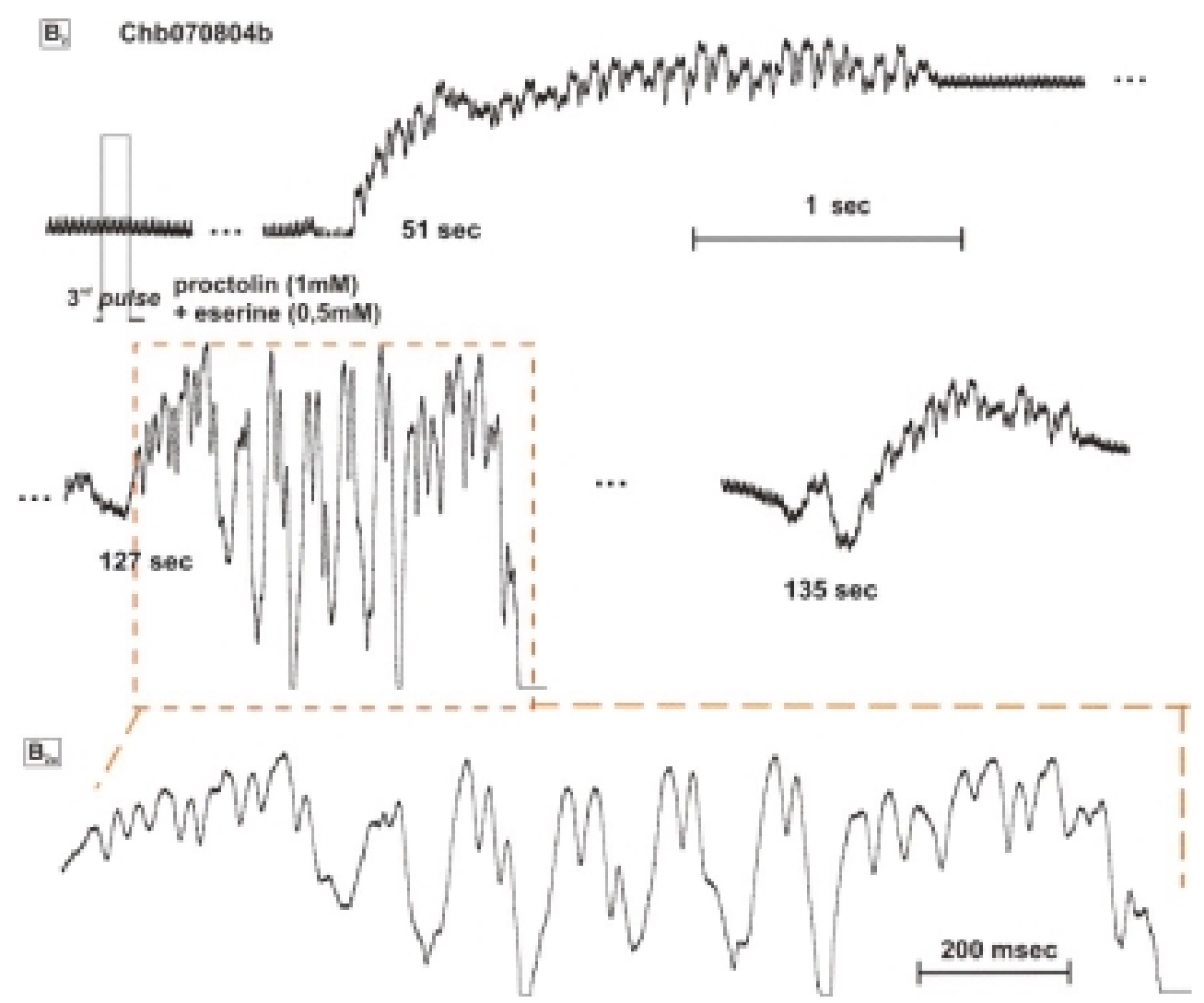

Fig. 3-33 $B_{2}$. Specific and unspecific stridulatory movements in response to the $3^{\text {rd }}$ pulse proctolin/eserine.

The stridulation elicited by the following proctolin pulse after eserine application, lasted over $10 \mathrm{~min}$ (Fig. 3-33, C). The response began with unspecific movements consisting stridulatory-like elements (Fig. 3-33, $C_{1}$ ). Sequences of $1^{\text {st }}$ order ("burst"-like) were performed regularly every $5-10 \mathrm{sec}$ (Fig. 3-33, $\mathrm{C}_{3}$ ). Occasionally in between the bursts-like sequences were produced unspecific up- and downmovements with stridulatory elements. The single sequences $1^{\text {st }}$ order $(1-2 \mathrm{sec})$ were performed within the "bursts" (up position of the hindlegs, 3-4 sec). The internal structure of a single "burst" and sequence $1^{\text {st }}$ order is shown in Fig. 3-33, $C_{3 b}$. 


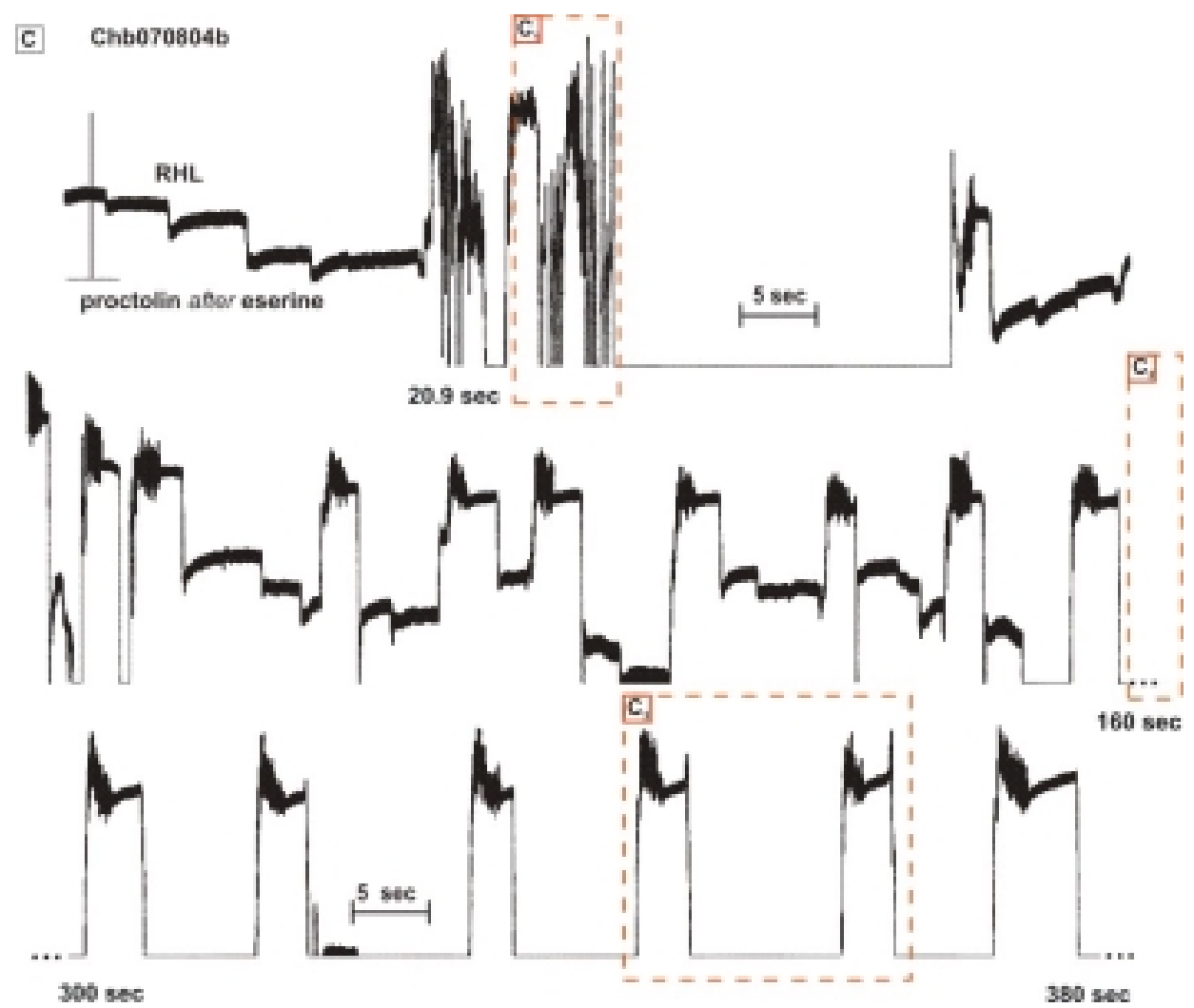

Fig. 3-33 C. Prolonged stridulation, stimulated by proctolin after eseine co-application (Ch.b.).
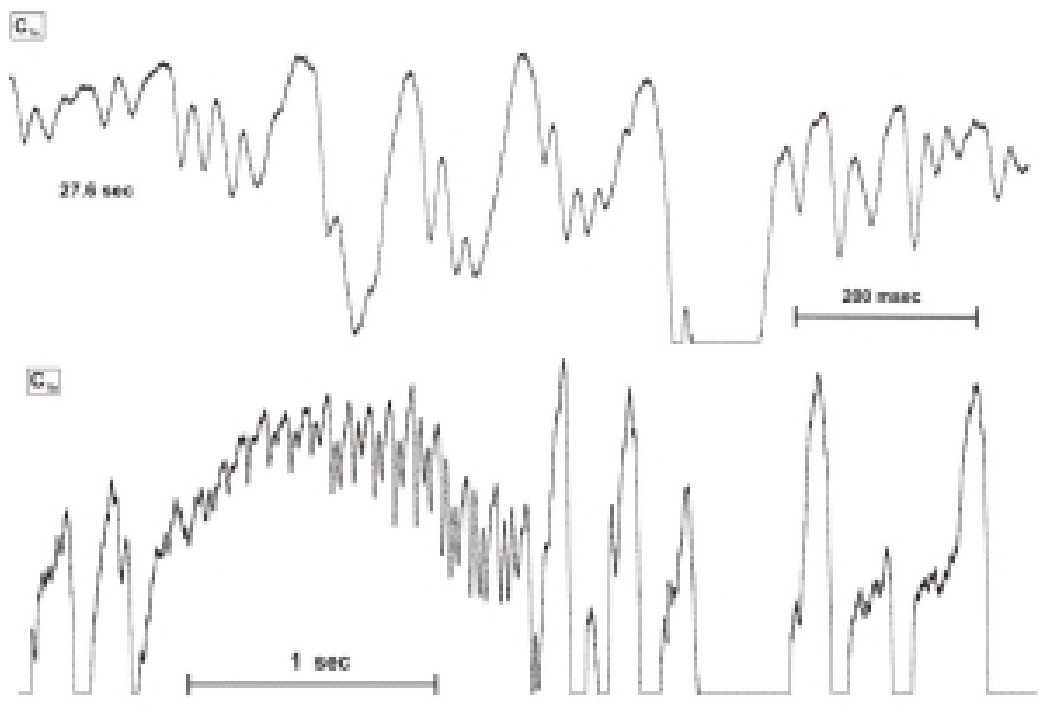

30 see

Fig 3-33 $\mathrm{C}_{1}$. Unspecific up- and down- movements, consisting stridulatory-like elements. 


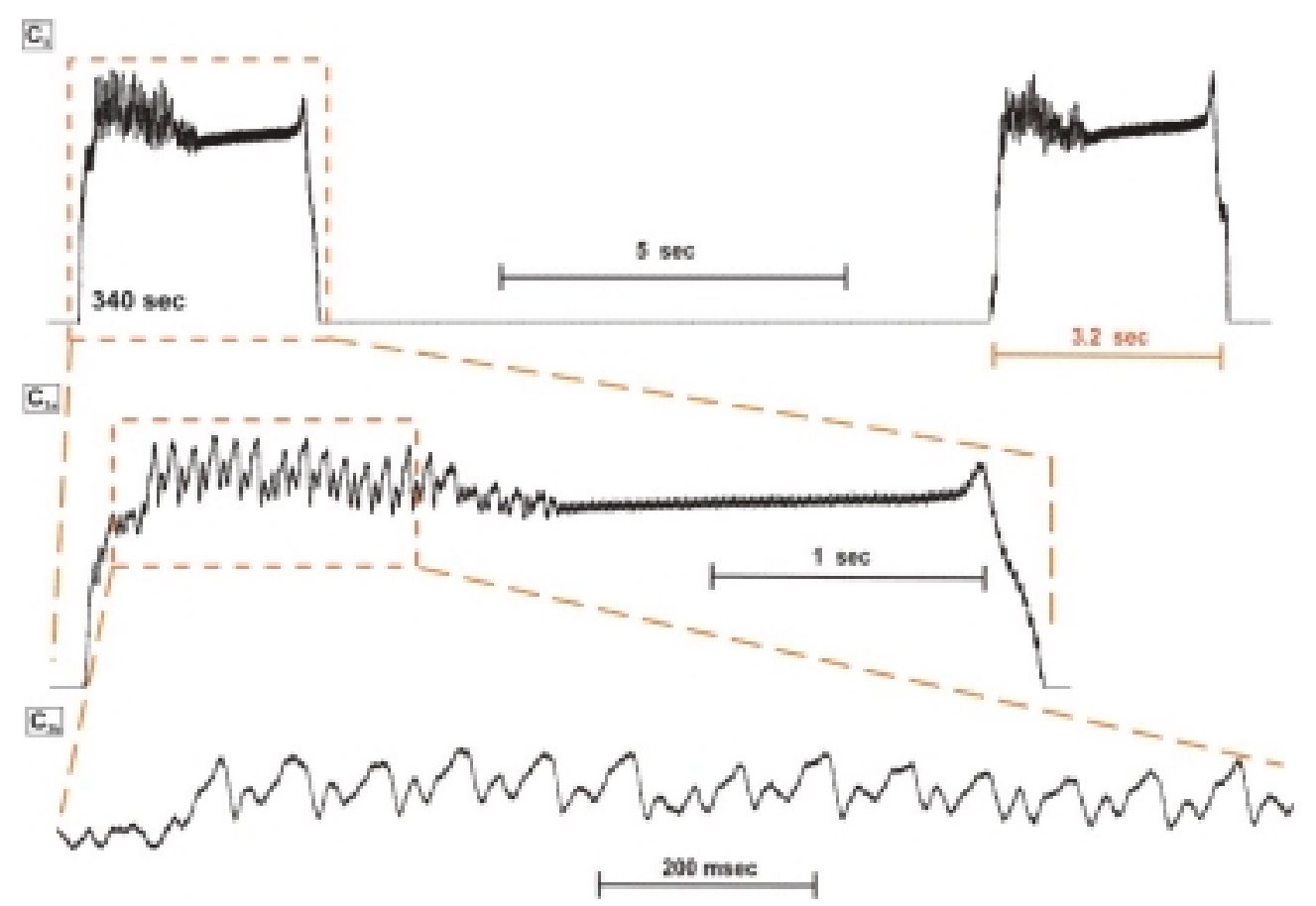

Fig. 3-33 $C_{3}$. „Burst-like“ sequences 1 st order performed by the hind legs. They were lifted 3-4 sec and produced 1-2 sec stridulatory movements $\left(\mathbf{C}_{3 \mathbf{b}}\right)$. After the stridulatory movements the hindlegs remained "silent" at up position $\left(\mathbf{C}_{3 \mathrm{a}}\right.$ ). Than the hindlegs were bring down suddenly (180-200 msec) preceding by an initial fast lifting $(60-80 \mathrm{msec})$ and were kept in down position $5-10 \mathrm{sec}\left(\mathbf{C}_{3}\right)$. 
Eserine also affected the muscarine-induced stridulation in another male grasshopper Ch.b. in a very similar manner. Table 3-2 presents the latencies and durations of the muscarine-stimulated responses before, during and after eserine co-injections.

\section{Table 3-2:}

\begin{tabular}{|c|c|c|c|c|c|}
\hline $\begin{array}{c}\text { Time (in } \\
\text { min) }\end{array}$ & $\begin{array}{c}\text { Protocol 3-3-3 } \\
\text { (No of pulses): }\end{array}$ & Injection of: & $\begin{array}{c}\text { Latency } \\
\text { (in sec): }\end{array}$ & $\begin{array}{c}\text { S Duration } \\
\text { (in sec): }\end{array}$ & $\begin{array}{c}\text { C Duration } \\
\text { (in sec): }\end{array}$ \\
\hline 0 & $1^{\text {st }}$ & Muscarine & 3,36 & 15,29 & 26,39 \\
\hline 4 & $2^{\text {nd }}$ & Muscarine & 4,12 & 13,78 & 25,49 \\
\hline 8 & $3^{\text {rd }}$ & Muscarine & 4,34 & 11,58 & 19,98 \\
\hline 12 & $1^{\text {st }}$ & Muscarine+ Eserine & 20,01 & 2,54 & 2,54 \\
\hline 16 & $2^{\text {nd }}$ & Muscarine+ Eserine & 16,4 & 10,14 & 149,82 \\
\hline 18 & $3^{\text {rd }}$ & Muscarine+ Eserine & 15,66 & 37,91 & 150,28 \\
\hline 22 & $1^{\text {st }}$ & Muscarine & 11,99 & $<240$ & $<600$ \\
\hline
\end{tabular}

Again, the initial muscarine stimulations elicited similar durations of stridulation occurring after latencies of approximately $4 \mathrm{sec}$ (Table 2). The latencies increased with application of muscarine/eserine mixture, the first of which elicited very short stridulation that increased gradually with third pulse up to 3 times compared to the initial muscarine stimulations. The following muscarine application induced singing of additionally prolonged durations that started after a medium latency. Fig. 3-34 A and B present the singing patterns, stimulated by muscarine before and after co-injection of eserine respectively.

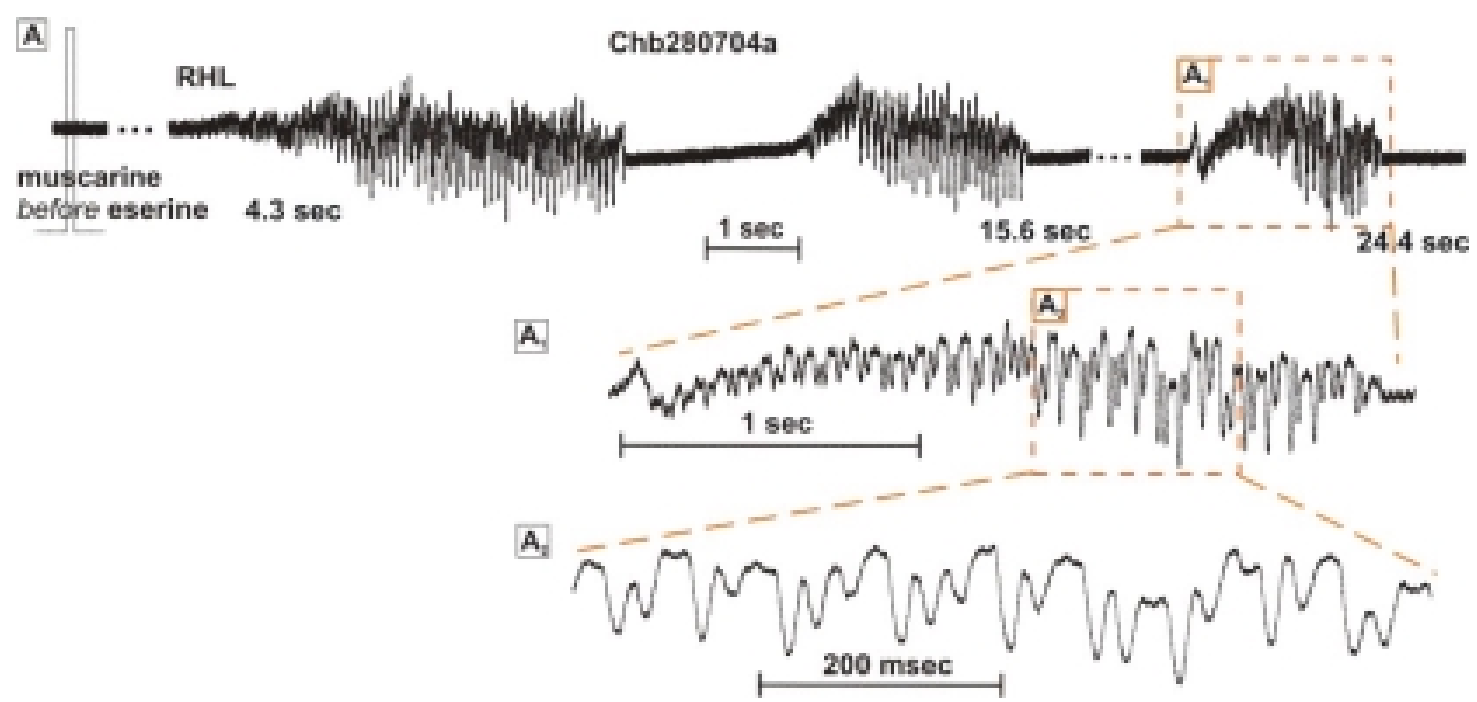

Fig. 3-34, A. Muscarine-induced singing before eserine co-injection (male, Ch.b.). $A_{1}$. Single sequence $1^{\text {st }}$ order; $A_{2}$. Internal structure of the sequence - chirps consisting 4 and 6 syllables 
The following to eserine co-injections muscarine pulse stimulated singing after $12 \mathrm{sec}$ latency, consisted of 15 sequences $1^{\text {st }}$ order, each 3-7 sec long, occurring every 5-10 sec. Stridulatory hindleg movements of low (Fig. 3-34, $\mathrm{B}_{1}$ ) and very high amplitude (Fig. 3-34, $\mathrm{B}_{2}$ ) were produced.

Similar to proctolin (Fig. 3-33, $\mathrm{C}_{3}$ ) muscarine produced also "burst"-like movement pattern (Fig. 3-34, $B_{2}$ ). Interestingly, in the case of muscarinic "bursts"

B

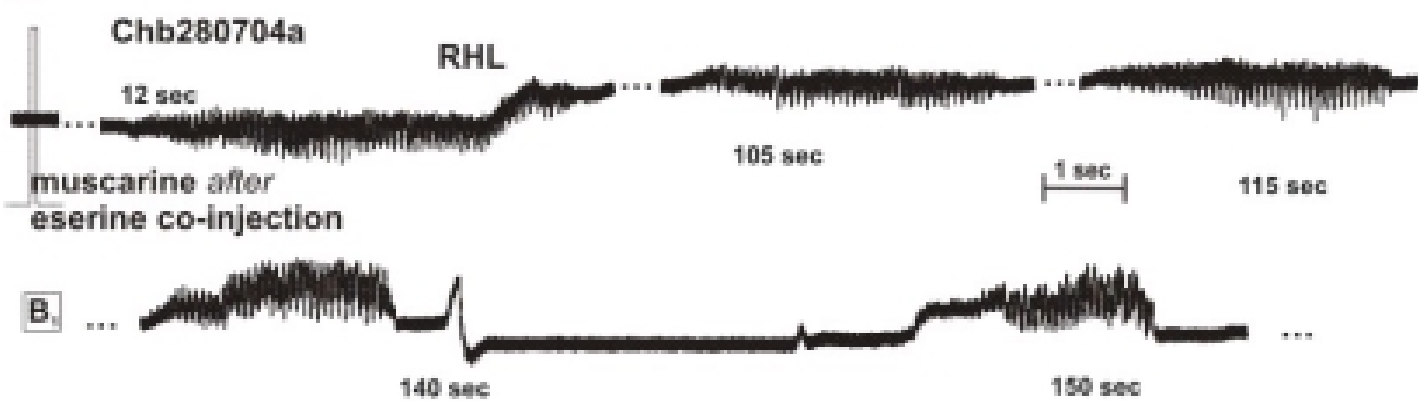

B.
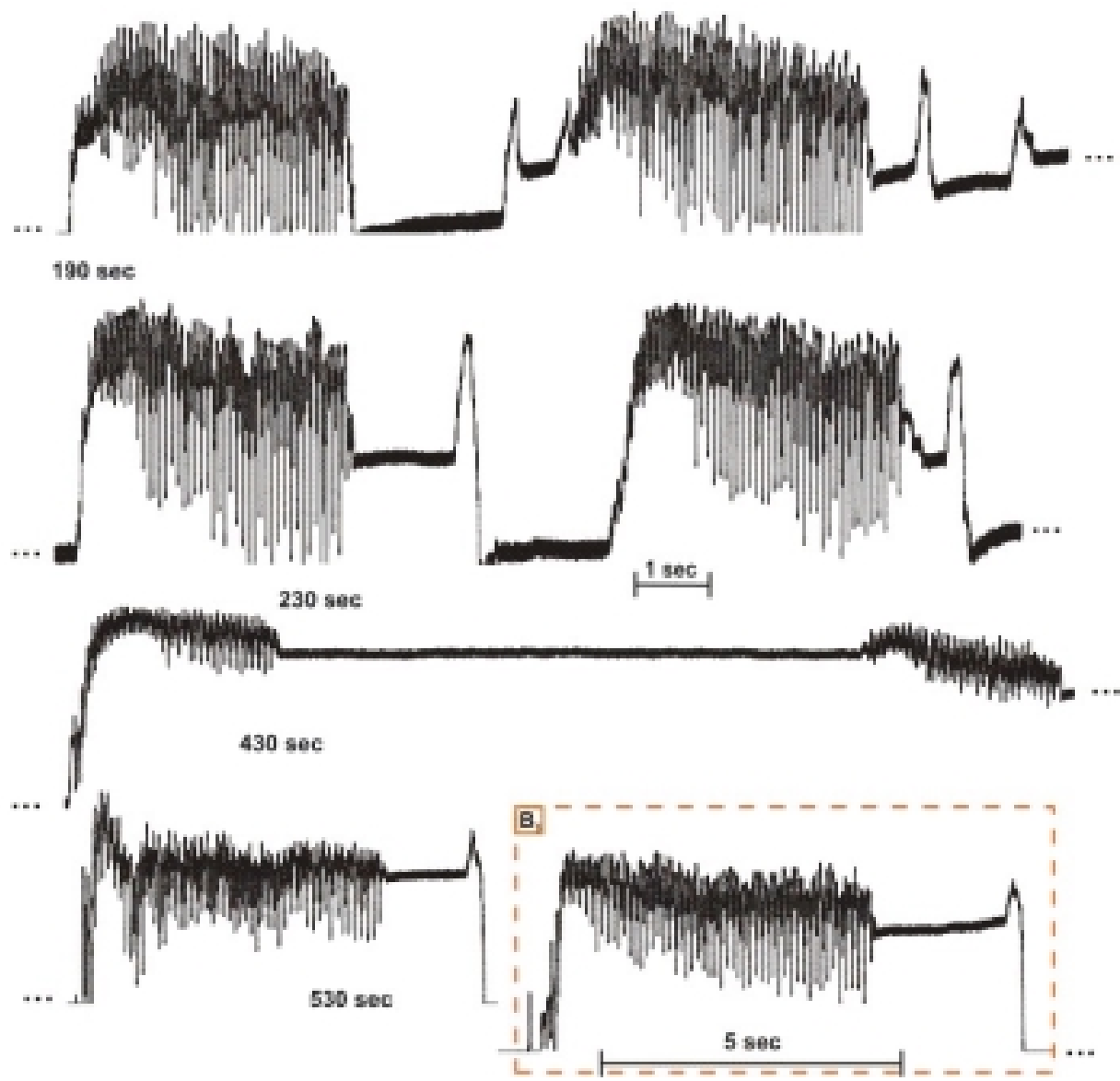

Fig. 3-34 B. Muscarine stimulation after eserine co-injections. A prolonged singing for 10 min was performed.

$B_{1}$. Stridulatory hindleg movements of low amplitude; $B_{2}$. Stridulatory movements of high amplitude;

$B_{3}$. "Burst"-like excitation was observed, similar to proctolin, after eserine co-injection (see Fig. 3-33, $C_{3}$ ) 
the up- position of the hind legs was kept 7-10 sec and the stridulatory movements lasted 5-8 sec (Fig. 3-34, $\mathrm{B}_{3}$ ). The proctolin-induced "bursts" was respectively 3-4 sec and 1-2 sec (Fig. 3-33, $C_{3 b}$ ).

During this very high level of excitation triggered by co-injection of eserine, again as it was shown in section 3.4.4 (Fig. 3-24), the muscarine-induced song sequences were 2-3 times longer than the relevant proctolin-induced song sequences.

The appearance of additional excitation to the muscarine- and proctolininduced ones, through inhibition of the AChE, suggests the additional mechanisms involved into the maintenance of the singing in Ch.b., besides mAChRs and PRs activation. A specific fine-tuned modulation of the AChE activity during the $\mathrm{ACh}$ and/or proctolin release may play

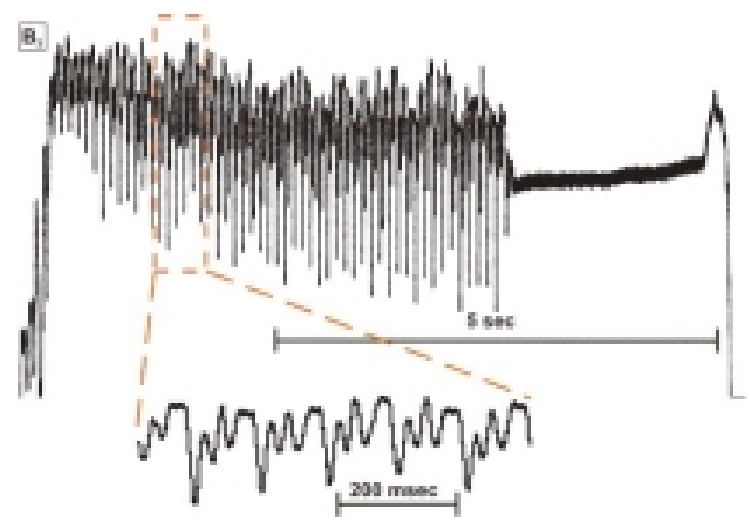

Fig. 3-34 $B_{3}$. Structure of the „burst“-like stridulatory hindlegs movements (see the text).

also a role in the cephalic control of singing in Ch.b. 
3.5 Intracellular pathways activated by proctolin receptors in the brain of Ch.b.

Typically, the actions of proctolin are slow in onset and prolonged, suggesting the involvement of a second messenger activated by a metabotropic receptor. Studies on various preparations revealed a number of candidate signaling pathways involving activation of adenylate cyclase (AC), activation of phospholipase $C$ (PLC), modulation of resting $\mathrm{G}_{\mathrm{K}}\left(\mathrm{K}^{+}\right.$membrane conductance) and other $\mathrm{K}^{+}$currents, release of $\mathrm{Ca}^{2+}$ from intracellular stores and $\mathrm{Ca}^{2+}$ entry via cell membrane channels.

Drugs that potentially interfere with proctolin receptors activated intracellular mechanisms and alter singing behavior in Ch.b. were applied in between two proctolin pulses, using protocol 3-1-4 or in combination with proctolin, using protocol 3-3-3. To identify the second messenger pathways mediating excitation following activation of PRs, substances known to permeate cell membranes and to alter the activity of certain second-messenger pathways were employed.

\subsubsection{The cAMP second messenger pathway}

Cyclic adenosine monophosphate is a second messenger that regulates a wide variety of cellular functions. Intracellular levels of cAMP are controlled by 2 distinct enzyme superfamilies: the adenylate (adenylyl) cyclases (AC), which use

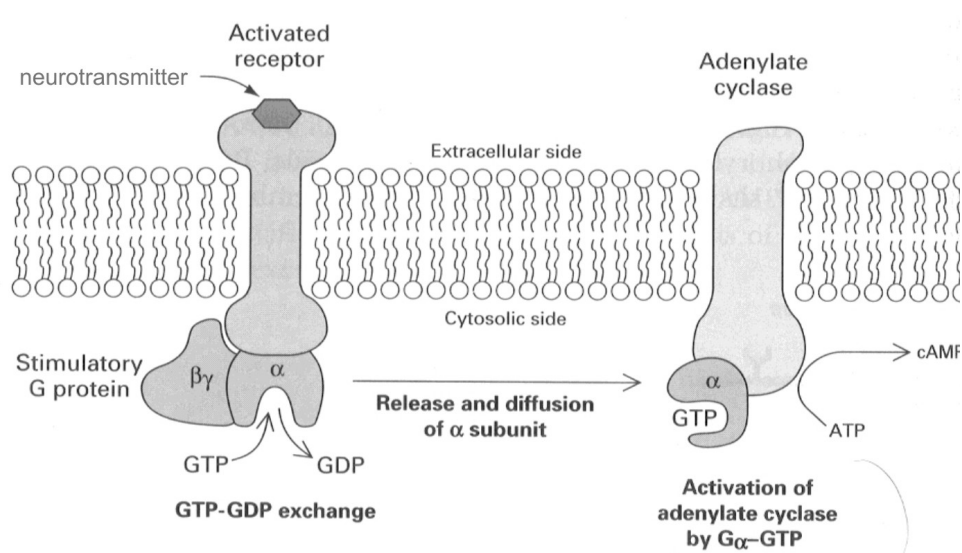

Fig. 3-35 AC activation, through the $\alpha$ subunit of $G_{s}$ proteins leads to CAMP synthesis. The degradation of CAMP to AMP is catalysed by PDE (not shown) (from Stryer, 1999). adenosine triphosphate 
(ATP) as a substrate to synthesize CAMP and the cAMP-specific phosphodiesterases (PDEs), which hydrolyze cAMP to biologically inactive adenosine 5'-monophosphate (Fig. 3-35).

Two main signal pathways, coupled respectively to excitatory $\mathrm{G}_{\mathrm{S}}$ and inhibitory $G_{i}$ and $G_{i / o}$ proteins (through their a subunits) regulate the level of cAMP by activation and inhibition of AC activity (Fig. 4-4, to the left and in the middle). AC is a $120-k D$ integral membrane protein with multiple transmembrane segments, which catalyzes the synthesis of CAMP.

Several experimental criteria have been used to determine whether cAMP serves as a second messenger in mediating the action of a particular neurotransmitter (Stryer, 1999):

1. AC in a target neuron should be stimulated by transmitters (or hormones) affecting that neuron. The AC activity of unresponsive cells should not be elevated by addition of the hormone.

2. The change in cAMP level in a target cell should precede or occur at the same time as the final outcome of hormonal stimulation. Variations in hormone levels should be matched by variations in the concentration of cAMP.

3. Inhibitors of the phosphodiesterase should act synergistically with hormones that employ cAMP as a second messenger.

4. The biological effects of a hormone should be mimicked by the addition of cAMP or a related compound to the targets neurons (in practice, CAMP cannot be readily used in this way because it penetrates cells poorly).

Experiments based on these criteria have revealed that cAMP is a ubiquitous second messenger for many transmitters and hormones: epinephrine, glucagons, calcitonin, chorionic gonadotropin, corticotropin, dopamine, follicle-stimulating hormone, lipotropin, luteinizing hormone, melanocyte-stimulating hormone, norepinephrine, parathyroid hormone, vasopressin and other vertebrate and invertebrates transmitters and hormones. 
Fig. 3-36 shows frequently used drugs, which influence the $G_{S}$ triggered cAMP cascade - activators (in green) and inhibitors (in red).

It has been demonstrated that the CAMP pathway is involved in the control of singing behavior in both Ch.b and O.v. initiated and sustained through muscarine activation of mAChRs (Heinrich et al., 2001; Wenzel, et al., 2002). Muscarineevoked stridulation has been partly blocked by inhibition of AC (through SQ22536)

\section{7 helix Receptors}

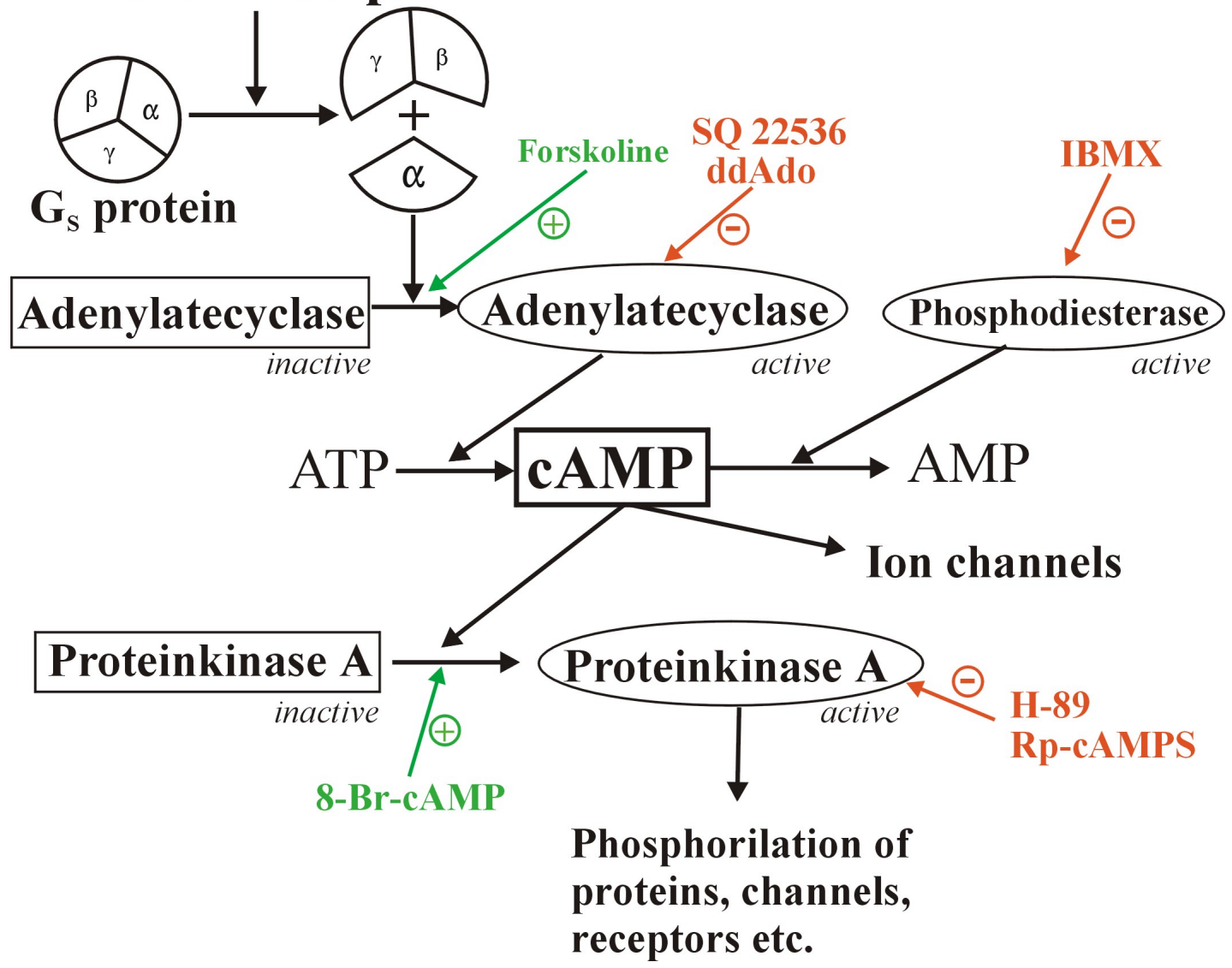

Fig. 3-36 cAMP second messenger pathway includes: metabotropic 7-helix receptor activation, $\mathrm{G}_{S}$ protein GTP-dependent hydrolysis, AC activation, cAMP synthesis and degradation and activation and/or modulation of effectors. Drugs available, which affect the cascade are shown: activators (in green) and inhibitors (in rot).

and PKA (through $\mathrm{H}-89$ ). Muscarine-sustained stridulation has been partly mimicked through AC activation (with forskoline), PDE inhibition (with IBMX) or activation of PKA (with 8-Br-cAMP and Rp-cAMPS). This suggested that mAChRs mediate excitation by stimulating the AC/cAMP pathway (Wenzel, et al., 2002). 
Application of drugs, affecting the cAMP second messenger pathway through microcapillary injection into brain neuropil of intact singing grasshopper does not fulfill the first criterion whether cAMP serves as a second messenger in mediating the action of mAChRs, simply because it could not be ensured that the cAMP cascade affected and mAChRs are on the same target neuron. A net increase of the cAMP level of the neuronal circuit, controlling singing behavior had been induced by muscarine, suggests that activation of mAChRs affects cAMP level directly or intermediated through consecutive activation of another receptors on the same or another neuron of the target neuropil. This pharmacological method is not sufficient to examine the direct coupling of a particular receptor to any second messenger cascade. It should be combined with further isolation of the neurons in culture and investigating the coupling of mAChRs to particular second messenger in a single neuron.

Only net contribution of particular signal pathway within the neuronal circuit mediating the proctolin-stimulated singing can be examined.

\subsubsection{Inhibition of AC through SQ22536 partly suppressed proctolin-} stimulated stridulation

In very early study proctolin has been shown to activate AC from both locust brain (Hiripi et al. 1979) and L. maderae hindgut (Wright, et al., 1986). In these studies also has been measured the net increase of cAMP in the brain. Other neuropeptides in many systems have been reported be coupled to this pathway (Nässel, 2002).

In a series of experiments the effect of AC inhibitor SQ22536 on proctolinstimulated singing behavior was evaluated. In 10 preparations tested, either reversible inhibition or no detectable effect on the duration of proctolin-stimulated singing occurred. The cumulative evaluation of all experiments shown in Fig. 3-37 reveals a weakly significant reduction of stridulatory activity 6 min after the application of the inhibitor.

The latency of the stridulatory responses was not affected (SD in range of $15 \%$ ) and the $C$ Duration did not differ from the S Duration, presented in the figure. 


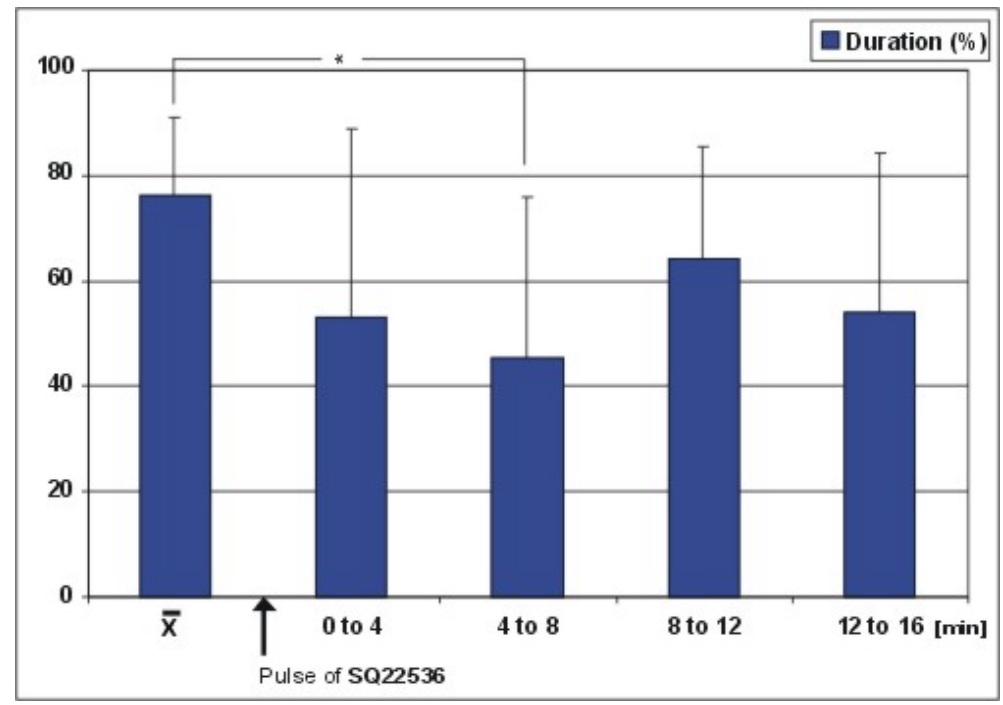

Fig. 3-37 Partial inhibition of proctolin-elicited stridulation through the AC inhibitor SQ22536 (male, Ch.b.; $\mathrm{n}=10 ; \mathrm{P}<\mathbf{0 , 0 5}$ ). The values of $\mathrm{S}$ Duration (\%) are presented.

This data suggest that proctolin mediates at least a portion of its stimulating effect on stridulation via activating the cAMP second messenger pathway. Thus the net level of cAMP in the neurons, contributing into proctolin-stimulated singing increases during the ongoing PRs activation in male Ch.b. 


\subsubsection{The PLC second messenger pathway}

Another ubiquitous cascade that is mediated through $\mathrm{G}$ proteins and evokes many responses is the phosphoinositide cascade or simply PLC cascade. Likewise the other cascades, it converts extracellular signals into intracellular ones. The intracellular messengers formed by activation of this pathway arise from phosphatidyl inositol 4,5-biphosphate $\left(\mathrm{PIP}_{2}\right)$, a phospholipid in the plasma membrane. The binding of a neuromodulator or neurohormone, for instance proctolin, to a cell-surface receptor (PRs) leads to activation of $\mathrm{Gq} / 11$ protein (GTP-binding). The $\alpha$-subunit of this $G$ protein activates phospholipase $C$ (PLC). Two messengers - inositol 1,4,5-triphosphate $\left(\mathrm{IP}_{3}\right)$ and diacylglycerol (DAG) - are formed by the cleavage of $\mathrm{PIP}_{2}$. Several kinds of mammalian phospholipase $\mathrm{C}-\alpha$, $\beta, \gamma, \delta$ etc. have been studied in detail. PLCs are cytosolic enzymes that act on membrane-inserted phophoinositide substrates. Their enzymatic activity increases markedly when the $\mathrm{Ca}^{2+}$ level is raised. Gq protein increases the catalytic activity of the $\beta 1$ isoform of PLC and increases its affinity for $\mathrm{Ca}^{2+}$ (Berridge, 1993; Nishizuka, 1992). $\mathrm{Li}^{+}$widely used to treat manic-depressive disorders, may act by inhibiting the recycling of $\mathrm{PIP}_{2}$. By few exceptions, for instance PLCY1, the isozyme is activated by receptor tyrosine kinase rather than by a $\mathrm{G}$ protein (Rhee and Choi, 1992).

$\mathrm{IP}_{3}$ opens channels to release $\mathrm{Ca}^{2+}$ from intracellular stores. It has been found that $\mathrm{IP}_{3}$ causes the rapid release of $\mathrm{Ca}^{2+}$ from the endoplasmic reticulum and, in smooth muscle cells, the sarcoplasmic reticulum through $\mathrm{IP}_{3}$-gated $\mathrm{Ca}^{2+}$ channels (Berridge, 1993). The elevated level of $\mathrm{Ca}^{2+}$ in the cytosol then triggers processes such as smooth muscle contraction, glycogen breakdown, transmitter release and many others. The highly cooperative opening of $\mathrm{Ca}^{2+}$ channels by nanomolar concentrations of $\mathrm{IP}_{3}$ enables neurons to detect and amplify vary small changes in the concentration of this messenger. Furthermore, $\mathrm{IP}_{3}$ is a short-lived messenger because it is rapidly converted into derivatives that do not open the channel. The lifetime of $\mathrm{IP}_{3}$ in most cells is less than a few seconds and in olfactory neurons is short as $100 \mathrm{msec}$ (Boekhoff et al., 1990). 
DAG, the other messenger formed by the GPCRs-triggered hydrolysis of $\mathrm{PIP}_{2}$ through PLC, activates protein kinase C (PKC). This multifunctional PKC phosphorylates serine and threonine residues in many target proteins. It has been found that PKC is enzymatically active only in the presence of $\mathrm{Ca}^{2+}$ and phosphatidyl serine. DAG increases the affinity of $\mathrm{PKC}$ for $\mathrm{Ca}^{2+}$ and thereby renders it active at physiologic levels of this ion (Nishizuka, 1992). DAG, like $\mathbb{I P}_{3}$, acts transiently because it is rapidly metabolized.

It has previously been reported that the $\mathrm{PLC} / \mathrm{IP}_{3} / \mathrm{DAG}$ signal transduction cascade (Fig. 3-38) mediates the performance of singing behavior in grasshoppers following activation of mAChRs. Inhibition of PLC activity by U-73122 and neomycin, which act by different mechanisms can completely suppressed muscarine-stimulated stridulation (Wenzel et al., 2002).

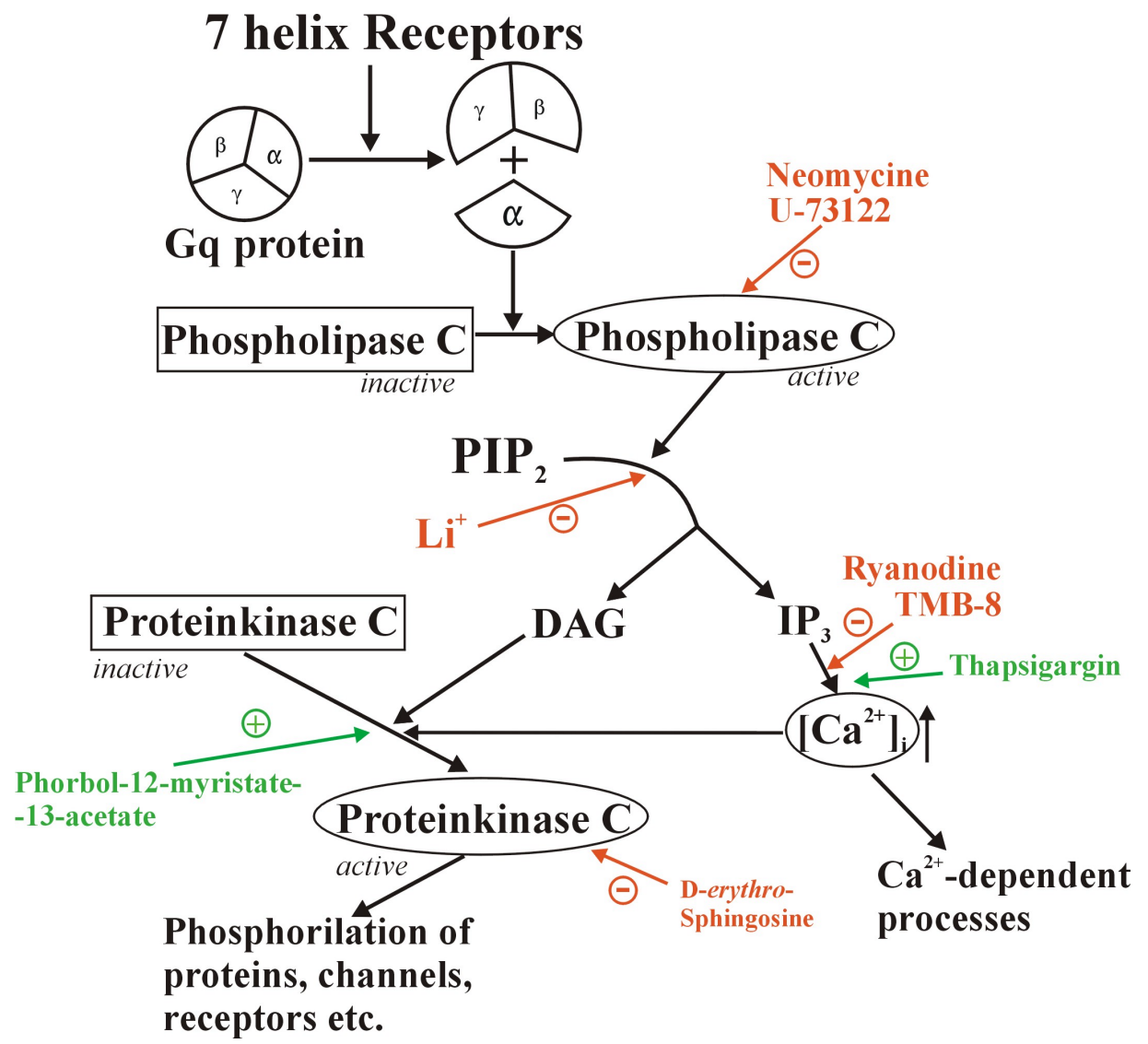

Fig. 3-38 PLC signal transduction cascade and frequently used activators (in green) and inhibitors (in red). 


\subsubsection{Blockade of PLC signal pathway through neomycin}

To investigate a possible involvement of phosphoinositides in mediating the proctolinergic excitation, neomycin, a substance that reduces PLC activity by binding to the enzyme's substrate PIP $_{2}$ (Shibanaka et al., 1993) was injected to site where proctolin stridulation. Injection of this inhibitor produced a gradually reversing reduction of proctolin-stimulated stridulation. The average duration of singing in all experiments decreased significantly $(P<0,05, n=10)$ in the period of 12-16 min (Fig. 3-39) In 3 other experiments the proctolin-initiated stridulation was suppressed irreversible.

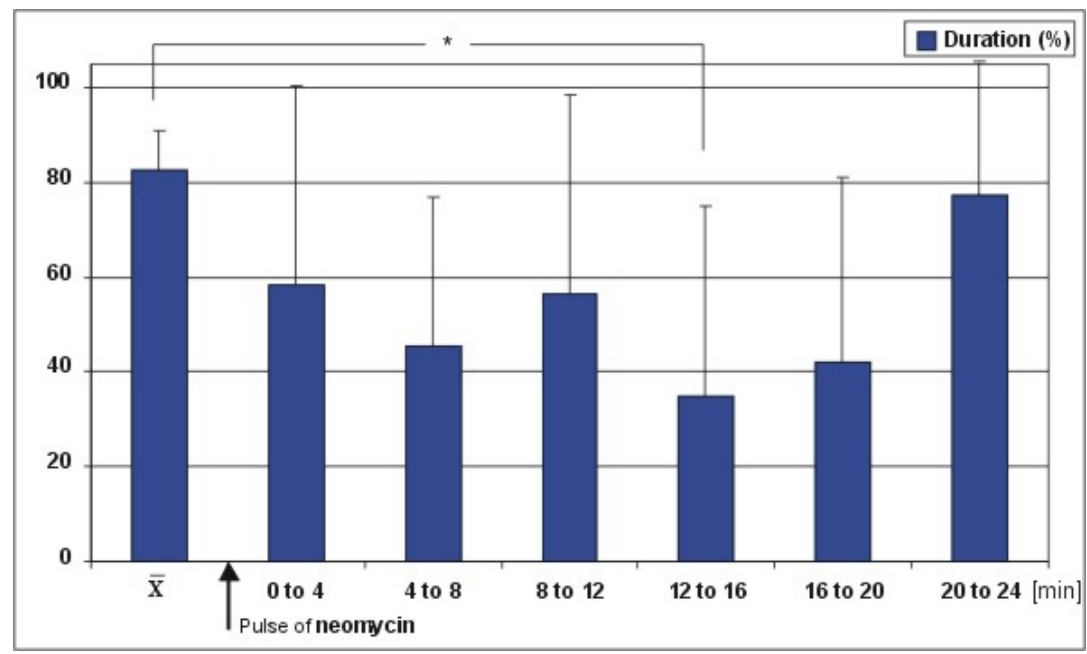

Fig. 3-39 Effect of PLC the inhibitor neomycin on proctolin-stimulated singing (males, Ch.b., $\mathbf{n}=10$ ). Two phases of reversable inhibition were observed: the first one to $10^{\text {th }} \mathrm{min}$ and/or the second from $10^{\text {th }}$ to $20^{\text {th }} \mathrm{min}$.

The long period of reduced stridulation shown in Fig. 3-39 resulted from different time courses in different preparations. In 6 of 10 experiments, stridulation was completely suppressed during the period 10-18 min. In 2 preparations, inhibition was fully developed between 2 to $10 \mathrm{~min}$ before reversing to control level duration. Another 2 experiments showed clear but irregularperiod of reduced stridulatory activity. Although a clear inhibitory effect appeared in all experiments, its variations with respect to the time of neomycine application, caused rather large values for SD, which in turned resulted in a rather weak level of significance in the evaluation of all experiments. Nevertheless, the robust appearance of the inhibitory 
effect of neomycin suggested a main role for the PLC pathway mediating proctolinergic excitation in Ch.b.

\subsubsection{Inhibition of the PLC signaling pathway through $\mathrm{Li}^{+}$}

$\mathrm{Li}^{+}$has been successfully used in mammalians to demonstrate the link between $\mathrm{M}_{3}$ receptor activation and $\mathrm{ACh}$-induced $\mathrm{PIP}_{2}$ hydrolysis (Hinton et al., 1998). Trimmer and Berridge (1985) reported that both $\mathrm{Li}^{+}$and atropine inhibited ACh-induced inositol phosphate production in the metathoracic ganglion of locusts, thereby suggesting the presence of a class of muscarinic receptors linked to phosphatidylinositol hydrolysis in insects. $\mathrm{Li}^{+}$, an inhibitor of several enzymes in the phosphoinositol cycle, has been used to demonstrate the coupling of GPCR to PLC signaling in many studies. However application of $\mathrm{Li}^{+}$leads either to an accumulation or a reduction of $\mathrm{IP}_{3}$ and DAG (Nahorski et al., 1991), depending on the mode of application. $\mathrm{Li}^{+}$has caused, for instance, slightly increase of proctolininduced contraction of muscle in P. americana (Wegener and Nässel, 2000). No matter the effect of $\mathrm{Li}^{+}$, either accumulation or reduction of $\mathrm{IP}_{3}$ and DAG level, is used as indication of a linkage between receptor activated and PLC signaling (Hinton et al., 1998; Wegener and Nässel, 2000).

In a series of experiments, $\mathrm{K}^{+}$in the saline used to dissolve the proctolin was substituted by $\mathrm{Li}^{+}$to give a $40 \mathrm{mM}$ concentration of $\mathrm{Li}^{+}$. It is believed that no ionic and osmotic effects could be caused by addition of $\mathrm{Li}^{+}$up to $100 \mathrm{mM}$ (Hinton et al., 1998).

In 3 from 10 experiments, a gradually increasing reversible reduction in the duration of proctolin-stimulated stridulation through $\mathrm{Li}^{+}$occurred (Fig. 3-40, A, B). No variations in the latencies could be detected (data not shown). In the rest 7 experiments, proctolin-stimulated stridulation was completely suppressed and did not recover during the following three injection of proctolin (data not shown).

Whether the lack of $\mathrm{K}^{+}$in $\mathrm{Li}^{+}$-containing proctolin solution might influence proctolin-stimulated stridulation will be examined in the following section. 

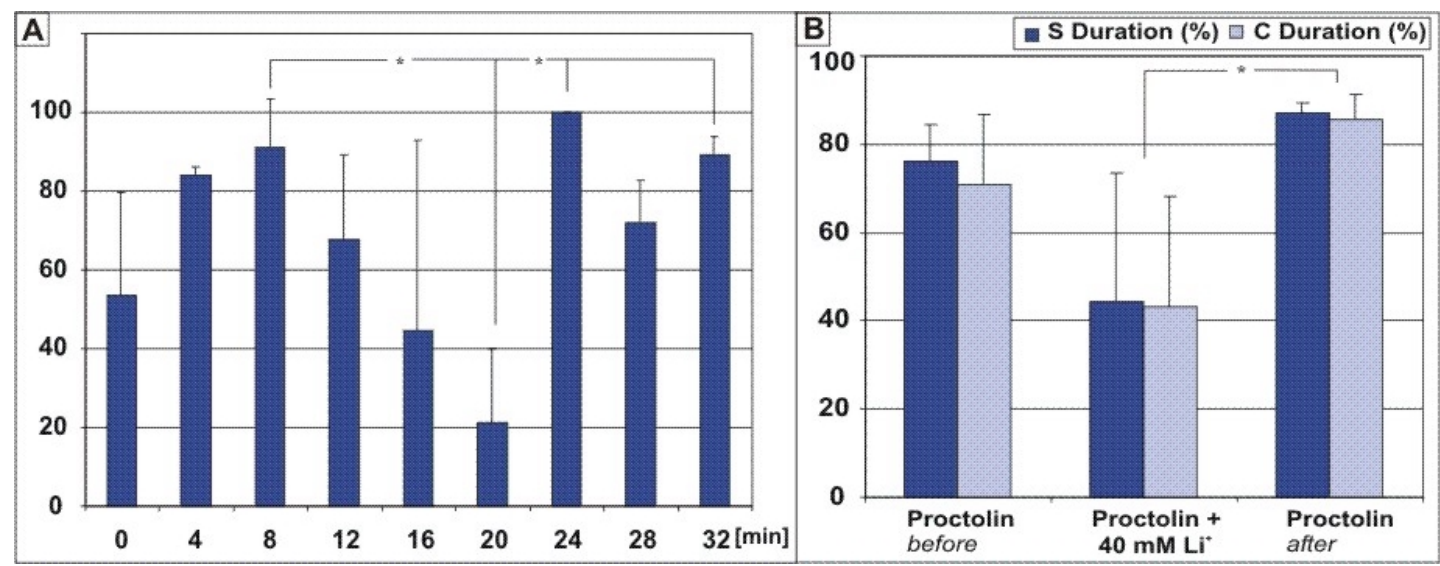

Fig. 3-40 $\mathrm{Li}^{+}(40 \mathrm{mM})$, a potent PLC inhibitor, reversible reduced proctolin-stimulated singing (males, Ch.b., n=3); A. Protocol 3-3-3: 0,4,8 and 24,28,32 min - pulses proctolin and 5 mM K ; $12,16,20 \mathrm{~min}$ - pulses proctolin and $40 \mathrm{mM} \mathrm{Li}^{+}$. B. Average values of the durations before, during and after $\mathrm{Li}^{+}$co-application. (In other 7 animals $\mathrm{Li}^{+}$caused an irreversible inhibition of the stridulation. These experiments wher not included into the analysis).

In another series of experiments $60 \mathrm{mM} \mathrm{Li}^{+}$was added to the saline instead of substituting it for $\mathrm{K}^{+}$. In all experiments $(\mathrm{n}=9)$ a reversible decrease of the duration of proctolin-stimulated singing occurred (Fig. 3-41, A, B). The average values of both durations $(S$ and $C)$ differed significantly $(P=0,016)$ between injections with or without $\mathrm{Li}^{+}$, while the latency was not affected (not shown).

Again a gradual decrease of the durations of stridulation in response to repetitive injections proctolin and $\mathrm{Li}^{+}$was recorded in all stimulations (Fig. 3-40 and
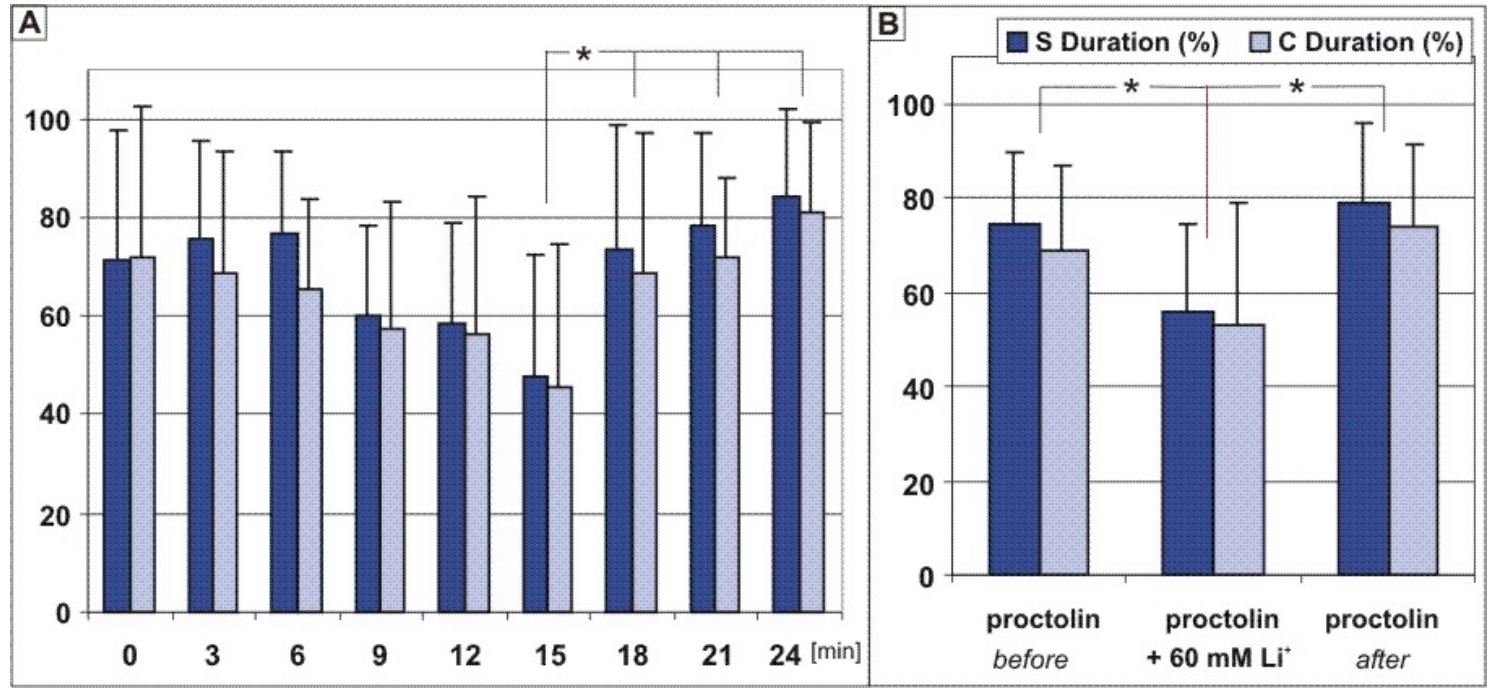

Fig. 3-41 Inhibitory effect of $\mathrm{Li}^{+}(60 \mathrm{mM})$ on PLC is lower in presence of $\mathrm{K}^{+}$in proctolin solution (males, Ch.b., n=9) A. protocol 3-3-3: 0,3,6 and 18,21,24 min - pulses proctolin; 9,12,15 min - pulses proctolin and $40 \mathrm{mM} \mathrm{Li}{ }^{+} ; \mathrm{B}$. Average values of the durations before, during and after $\mathrm{Li}^{+}$co-injection $(\mathrm{P}<0,05)$. 
Fig. 3-41). Thus, $\mathrm{Li}^{+}$, instead of lack of $\mathrm{K}^{+}$seemed to mediate the suppression of proctolin-stimulated stridulation, since the inhibitory effect was the same, whether $\mathrm{Li}^{+}$was added to the saline or replaced $\mathrm{K}^{+}$.

The effects of $\mathrm{Li}^{+}$further support the hypothesis that PLC signaling might play a role in the mediation of proctolin-induced excitation. Moreover, the PLC signaling seems to play a role in the accumulation of the excitation produced by the repetitive proctolin injections.

\subsubsection{Contribution of $\mathrm{Ca}^{2+}$ signaling to proctolin-mediated excitation}

The coupling of $\mathrm{Ca}^{2+}$ signaling to PRs activation has been well studied in smooth muscles of the locust ( $S$. gregaria) and cockroach ( $P$. americana). $\mathrm{Ca}^{2+}$ release from the sarcoplasmic reticulum plays a major role during proctolin-induced contractions in both model systems (Hinton et al., 1998; Wegener et al., 2000). Whether $\mathrm{Ca}^{2+}$ signaling is also coupled to PRs activation in insect CNS is not known.

\section{Intracellular $\mathrm{Ca}^{2+}$ release during proctolin stimultion}

TMB-8, an unspecific blocker of intracellular $\mathrm{Ca}^{2+}$ release, has been used to distinguish the release of $\mathrm{Ca}^{2+}$ from intracellular from extracellular $\mathrm{Ca}^{2+}$ influx in proctolin mode of action (Wegener and Nässel, 2000).
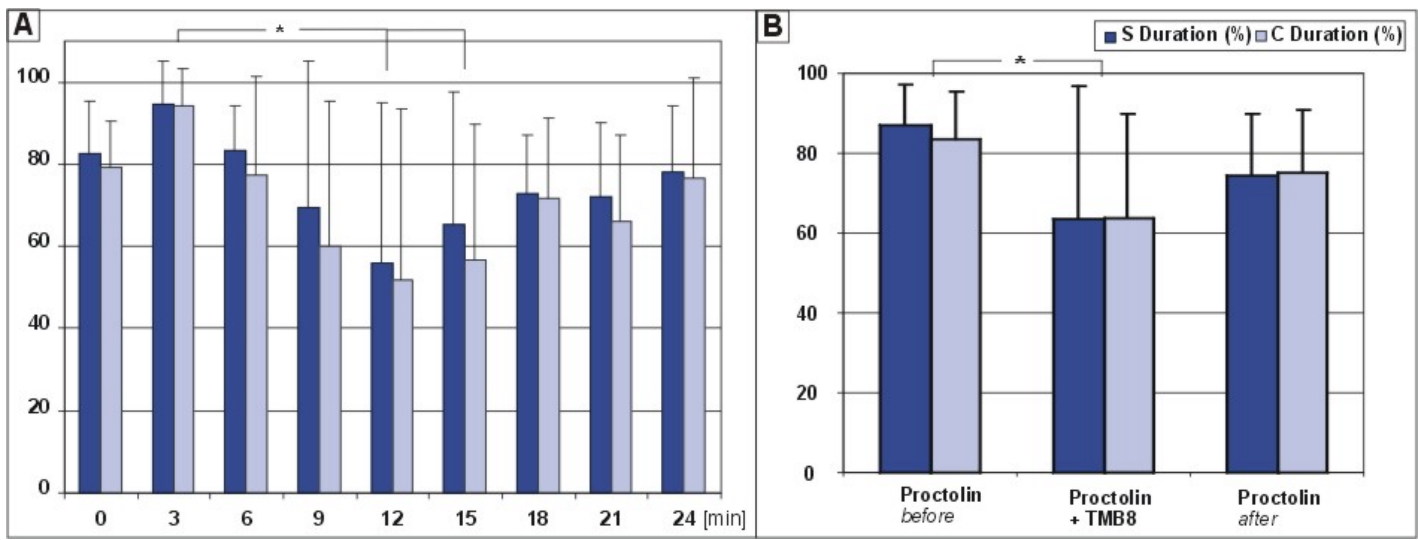

Fig. 3-42 TMB-8 decresed the durations of proctolin-stimulated stridulation (males, Ch.b. $n=6) A$. protocol 3-3-3: 0,3,6 and 18,21,24 - responses to proctolin; 9,12,15 - responses to proctolin and TMB-8 $(1 \mathrm{mM} ; 1: 1)$; B. Average values of the durations before, during and after TMB-8 co-injections 
TMB-8, applied with proctolin, decreased the durations of proctolin-stimulated stridulation from $86 \%$ to $63 \%(\mathrm{SD}=32 \%, \mathrm{P}<0,05, \mathrm{n}=6)$ (Fig. 3-42). In 2 experiments a complete but reversible inhibition was observed. Fig. 3-43 shows one of the recorded responses before (Fig. 3-43, A) and during (Fig. 3-43, B) TMB-8 coapplication. The inhibitory effect of this unspecific $\mathrm{Ca}^{2+}$ channel blocker indicated the involvement of $\mathrm{Ca}^{2+}$ release from intracellular stores for mediating proctolinstimulated singing in Ch.b.

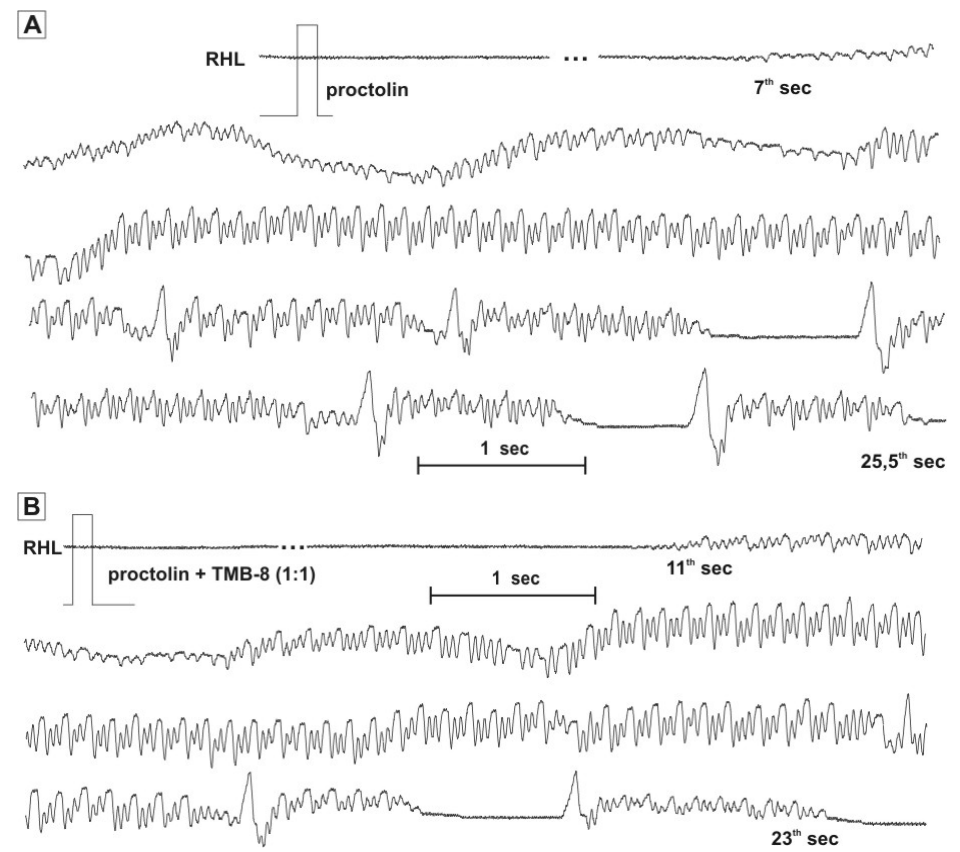

Fig. 3-43 Songs, produced by injection proctolin (A) and coinjection of proctolin and TMB-8 (B).

Thapsigargin known as a blocker of the sarcoplasmic $\mathrm{Ca}^{2+}$-ATPase (Treiman et al, 1998) has been reported to reduce proctolin-induced contractions of smooth muscles in $P$. americana (Wegener and Nässel, 2000). Thapsigargin empties the $\mathrm{Ca}^{2+}$ form intracellular stores by inhibiting its reuptake and the refilling of calcium stores (Zimmermann and Walz, 1997, 1999). After a period of increase of $\mathrm{Ca}^{2+}$ level in the cytosol it eventually blocks the responsiveness of the neuron via signaling pathways that involve $\mathrm{Ca}^{2+}$ release from intracellular stores.

In all 4 experiments conducted, using protocol 3-1-4, thapsigargin consistently prolonged proctolin-

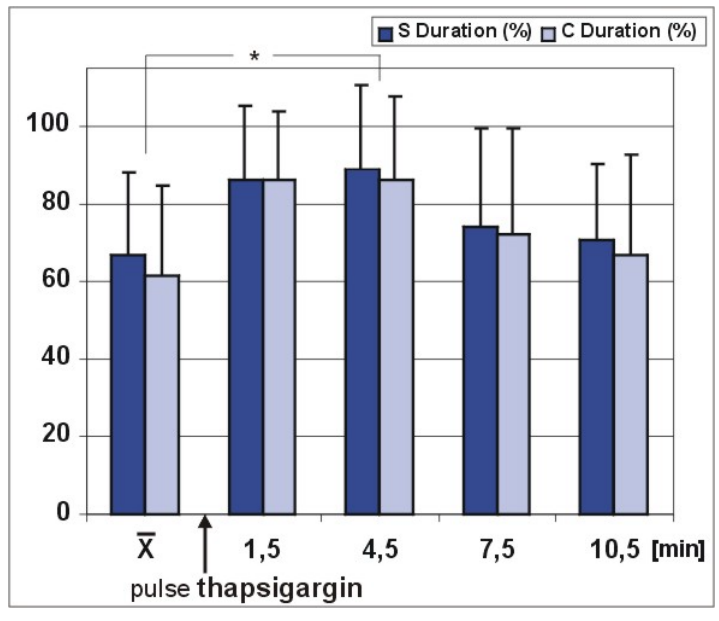

Fig. 3-44 Thapsigargin increased the durations of proctolin-induced stridulation (males, Ch.b., $n=4$, protocol 3-1-4) 
stimulated stridulation from $67 \%(S D=21,11 \%)$ to $89 \%$ (SD=21,77\%) (Fig. 3-44). The next Fig. 3-45 (A, B) shows proctolin-stimulated singing before and after thapsigargin injection. These results indicated that proctolin might use $\mathrm{Ca}^{2+}$ release from intracellular stores as a messenger signal but in a specific manner. A role of

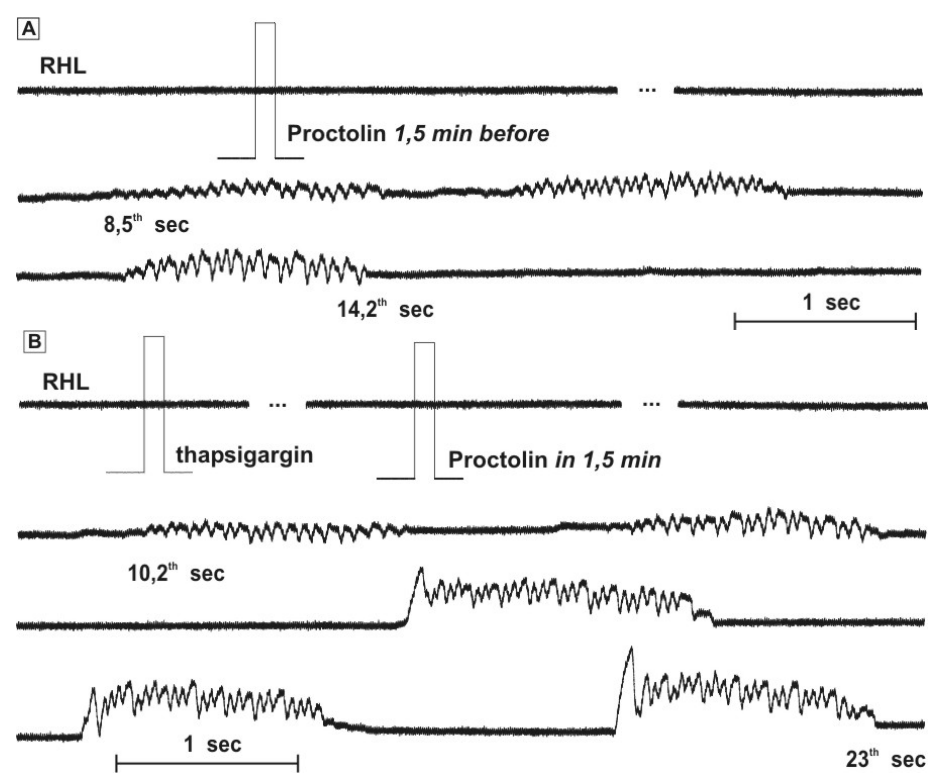
Fig. 3-45 Proctolin-iduced singing before and after thapsigargin injection (male, Ch.b.) thapsigargin on mAChRs mediated activation of singing has been not found (Wenzel et al., 2002). Moreover, thapsigargin did not elicit stridulation when applied alone (Wenzel et al., 2002).

The importance of intracellular release $\mathrm{Ca} 2+$ was further studied by employing ryanodine, an inhibitor of IP3-gated $\mathrm{Ca}^{2+}$ channels located in the membranes of intracellular stores.

\section{Contribution of $\mathrm{IP}_{3}$ and its receptors on internal $\mathrm{Ca}^{2+}$ stores to proctolin} mediated excitation

Ryanodine sensitive $\mathrm{IP}_{3}$-gated $\mathrm{Ca}^{2+}$ channels have been reported to play a
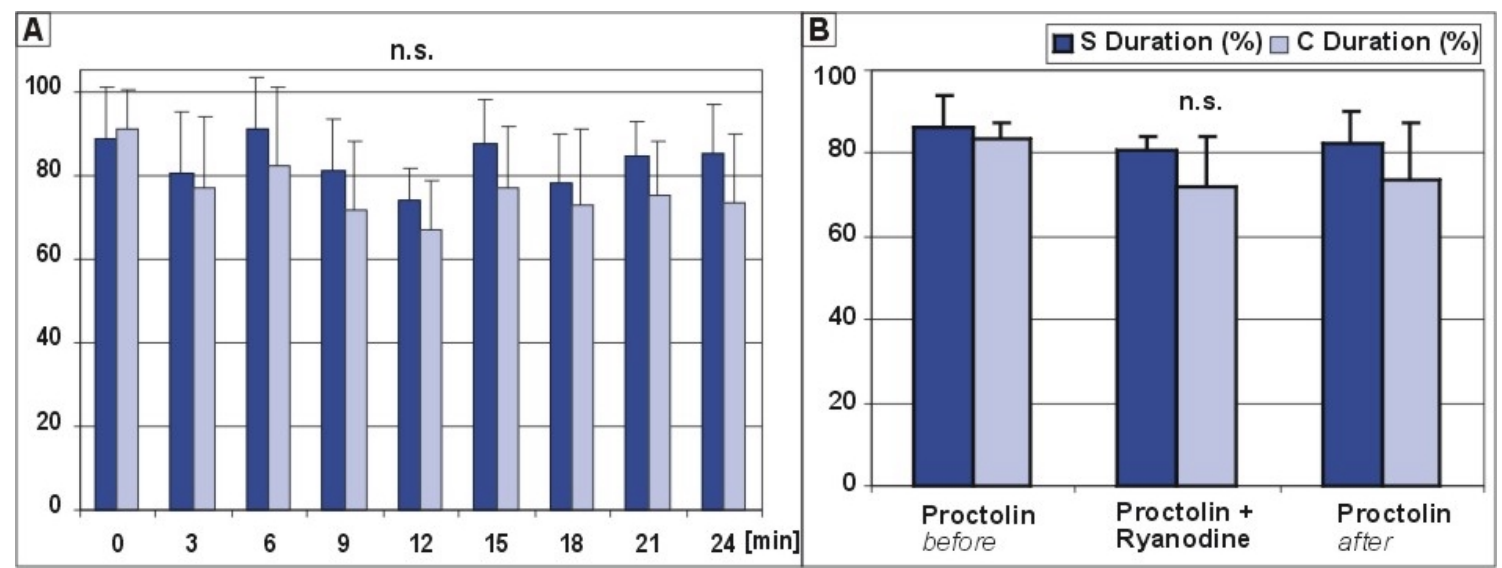

Fig. 3-46 Ryanodine failed to affect proctolin-induced singing (males, Ch.b., $n=5$ ) A. protocol 3-3-3: 0,3,6 and 18,21,24 $\mathrm{min}$ - responses to proctolin; 9,12,15 - responses to proctolin with ryanodine (1mM; $1: 1)$; B. Average values of the durations before, during and after ryanodine co-injections. 
central role in the cytosolic $\mathrm{Ca}^{2+}$ increase. They have been shown to mediate muscle contractions in locust (S. gregaria) foregut, induced by proctolin (Hinton et.al, 1998).

Ryanodine, applied in addition to proctolin, had no effect on proctolinstimulated stridulation (Fig. 3-46, A, B, n=5), neither on the durations, nor on the latency (not shown).

Although ryanodine did not affect the singing parameters in some case altered the singing patterns I and II within a single song sequences (Fig. 3-47).

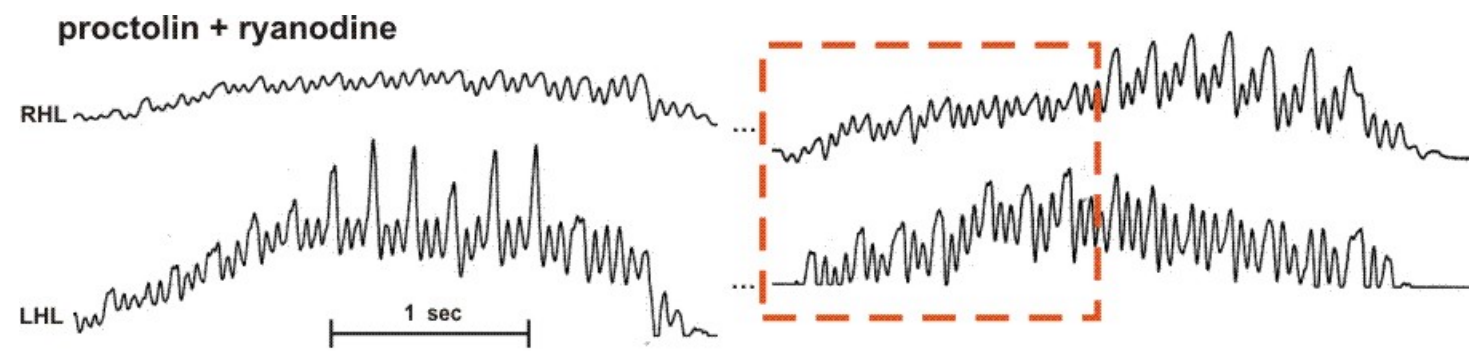

Fig. 3-47 Alteration of pattern I and II within a single song sequence by co-application of proctolin and ryanodine (male, Ch.b.)

However, since it cannot be estimated how well permeates through the membranes and accumulates in brain neurons of Ch.b., a contribution of ryanodine receptors to proctolin-triggered signaling cannot be completely ruled out.

\section{Extracellular $\mathrm{Ca}^{2+}$}

In many insect muscle preparations proctolin-evoked processes included activation and/or modulation of voltage- and/or ligand-gated $\mathrm{Ca}^{2+}$ channels and thus triggering depolarization or $\mathrm{Ca}^{2+}$-dependent $\mathrm{Ca}^{2+}$ release from intracellular $\mathrm{Ca}^{2+}$ stores (Wegener and Nässel, 2000; Washio and Koga, 1990; Hinton et al., 1998).

Proctolin has been shown to modulate the activity of 2 types of $\mathrm{Ca}^{2+}$ channels in the plasma membrane of a crayfish tonic flexor muscle. In this system proctolin serves as a cotransmitter in 3 of the 5 excitatory tonic flexor motoneurons and causes enhanced tension of the muscles after depolarization by the conventional neurotransmitter. Proctolin alone has had no effect on these channels. (Bishop et al., 1991). 
In series of experiments repetitive stimulation with proctolin and proctolin plus $5 \mathrm{mM} \mathrm{Ni}^{2+}$ were performed to examine the involvement of membrane $\mathrm{Ca}^{2+}$ channels. $\mathrm{Ni}^{2+}$ is known as a non-specific blocker of $\mathrm{Ca}^{2+}$ channels, affecting both voltage and ligand-gated channels. The results are shown in Fig. 3-48. The durations of proctolin-stimulated stridulation decreased gradually from $84 \%$ $(S D=4 \%)$ to $42 \%(S D=27 \%)(P<0,05 ; n=5)$ over the course of three applications of $\mathrm{Ni}^{2+}$. Similar to the effect of $\mathrm{Li}^{+}$, described above, the repetitive injections of proctolin with $\mathrm{Ni}^{2+}$ induced stridulation of gradually decreasing durations (Fig. 3-48, A).

This result suggests that proctolin may trigger intracellular signaling
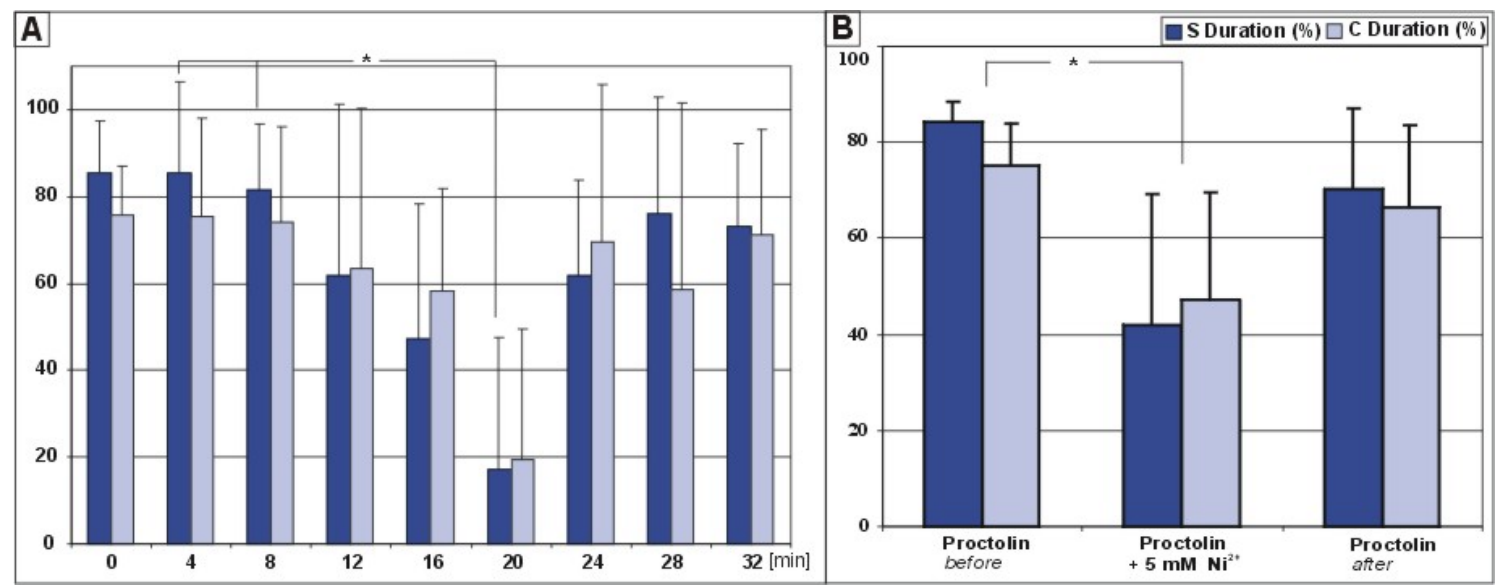

Fig. 3-48 The unspecific $\mathrm{Ca}^{2+}$-channels blocker $\mathrm{Ni}^{2+}$ reduced gradually the proctolin-maintained stridulation (males, Ch.b., $\mathrm{n}=5$, protocol 3-3-3).

cascades, that somehow lead to the opening of $\mathrm{Ca}^{2+}$ channels in the cell membrane to continue the progress of generating excitation. This interpretation must nevertheless be used with caution, since $\mathrm{Ca}^{2+}$ channels are crucially involved in synaptic transmission and the observations summarized in Fig. 3-48, B may as well result from impairment of synaptic transmission.

\subsubsection{Contribution of DAG to proctolin-stimulated stridulation}

In addition to IP3, DAG is the other second messenger generated through PLC activity. In various insect preparations (Baines and Downer, 1992; Hinton et al., 1998; Wegener et al., 2000) DAG has been shown to contribute to proctolin receptor-triggered excitation. 
A possible role of DAG in proctolin-stimulated stridulation was examined through injections of phorbol-12,13-dibutirate (phorbol ester, PhE, right) at the same brain areas of Ch.b., where proctolin stimulated singing.

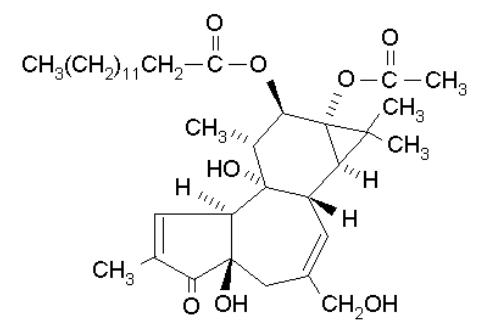

$\mathrm{PhE}$ was first dissolved in DMSO subsequently diluted with Ringer solution to a final concentration of $\mathrm{PhE} 0,4 \mathrm{mM}$ and $5 \%$ DMSO. The same concentration of DMSO was added to the control proctolin solution.

In the first series of experiments $\mathrm{PhE}$ was injected alone from the second chamber of the capillary following and preceding 3 proctolin pulses all injected with the same interval between individual stimulations (modification of protocol 3-3-3).
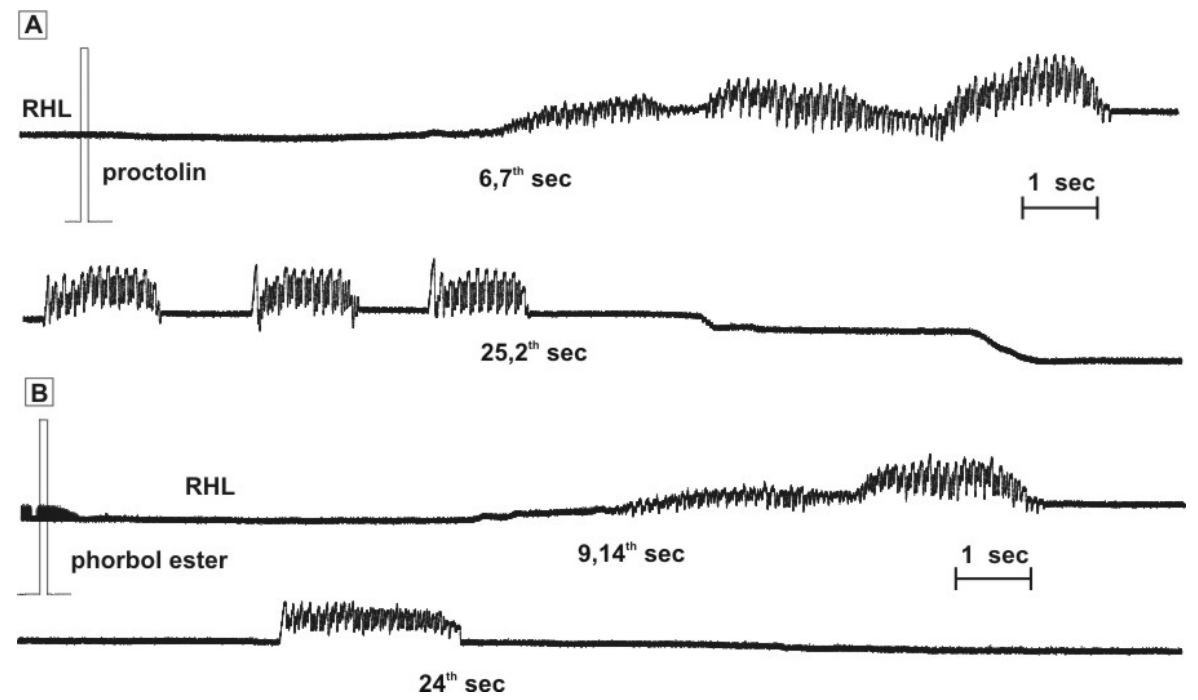

Fig. 3-49 Male songs (Ch.b.) induced through proctolin (A) and phorbol ester (B) at the same spot in the brain. 
Injections of $\mathrm{PhE}$ into the Ch.b. brain elicited coordinated stridulatory movements that were indistinguishable from proctolin-stimulated stridulation induced at the same site within the brain (Fig. 3-49, A, B). However, the time course of the released stridulation differed in that the average relative duration of stridulatory behavior after injection of PhE was significantly shorter (from $80 \%$, $\mathrm{SD}=9,77 \%$ down to $50 \%, \mathrm{SD}=23,88 \% ; \mathrm{P}<0,05 ; \mathrm{n}=4)$ than the preceding and following injections of proctolin (Fig. 3-50, A, B), while the latency was significantly longer (from $74 \%, S D=7,73 \%$ up to $97 \%, S D=5,21 \%$ )(Fig. 3-50, A, B).
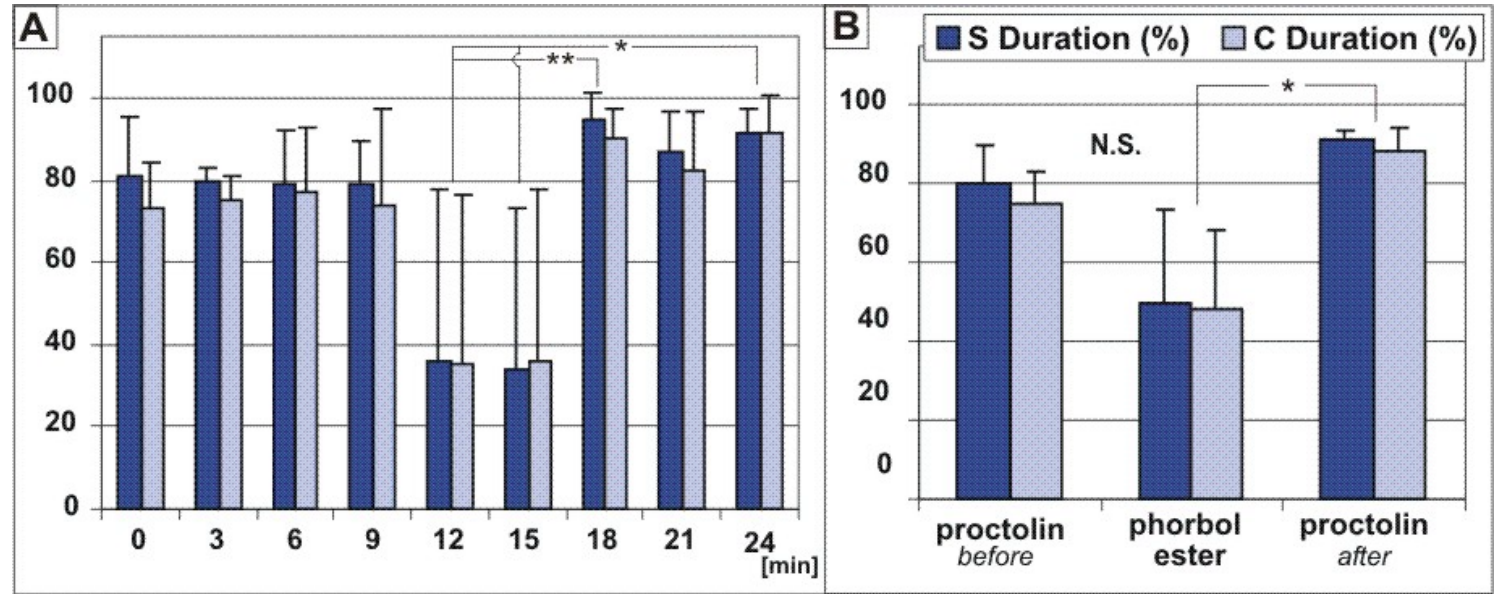

Fig. 3-50 The durations of proctolin- and phorbol ester-induced songs (males, Ch.b., $n=4) A$. protocol 3-3-3: 0,3,6 and 18,21,24 - responses to proctolin; 9,12,15 - responses to PhE; B. Average values of the durations $(P<0,05)$.

After 3 pulses $\mathrm{PhE}$ the durations of proctolin-elicited stridulation increased up to slightly higher levels than before (Fig. 3-51, A, B), indicating a weak after effect on the excitation due to PhE stimulation. 

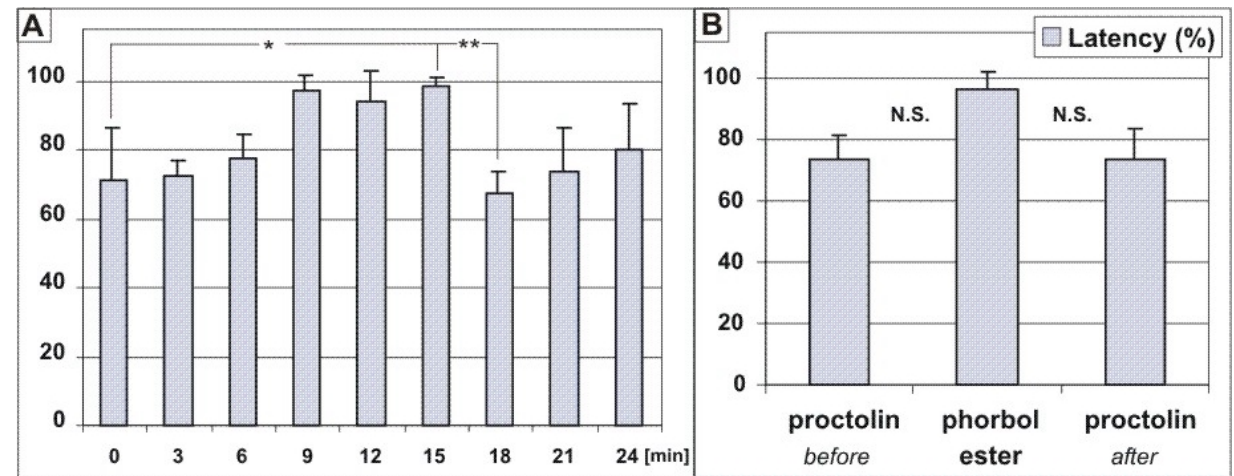

Fig. 3-51 The latencies of the stridulation induced by proctolin and phorbol ester (males, Ch.b., $n=4$ ) A. protocol 3-3-3: 0,3,6 and 18,21,24 - pulses proctolin; 9,12,15 - pulses PhE. B. Average values of the latencies.

To confirm the effect of $\mathrm{PhE}$ on the proctolinmaintained stridulation, $\mathrm{PhE}$ was injected in between two proctolin pulses, using protocol 3-1-4 (Fig. 3-52) Ch. b., $\mathrm{n}=7$ ). The duration of proctolin-stimulated singing differed significantly before and after the PhE pulse. The duration increased from an initial value of $67 \%$ $(\mathrm{SD}=19,8 \%) \quad$ to $\quad 83 \%$

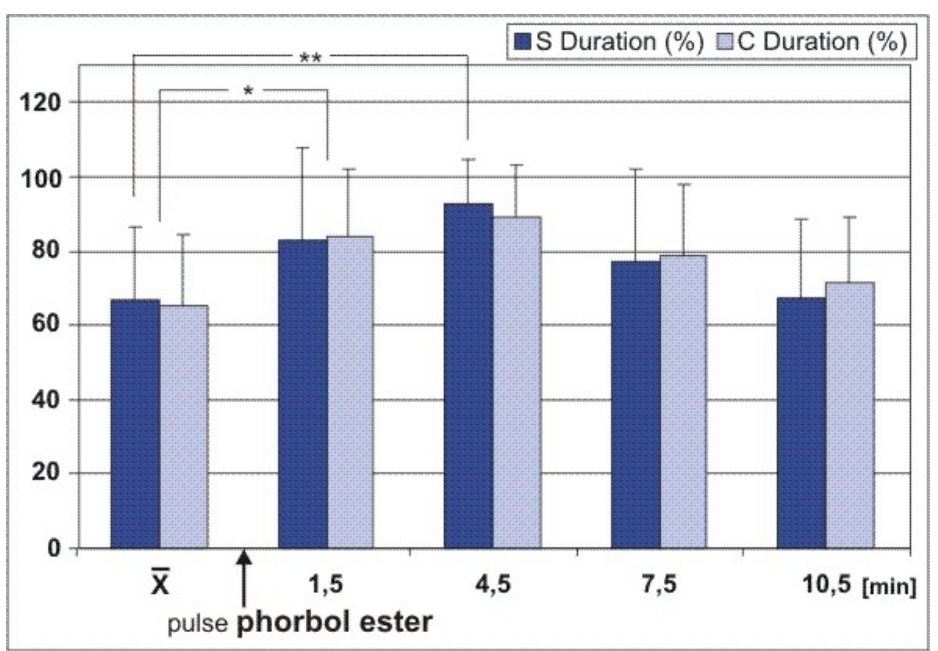

Fig. 3-52 Increasing of the durations of proctolin-induced songs through injection of phorbol ester (males, Ch.b., $n=7$; protocol 3-1-4) $(\mathrm{SD}=25 \%, \mathrm{P}<0,05)$ in response to the proctolin pulse, following $\mathrm{PhE}$ pulse (in 1,5 $\mathrm{min})$ and reached the maximal level of $93 \%(\mathrm{SD}=11,2 \%, \mathrm{P}<0,01)$ in response to the second proctolin pulse (in 3,5 $\mathrm{min}$ ) and than reversed to the initial level of $68 \%$ $(S D=20,8 \%)$ after $10,5 \mathrm{~min}$.

In another set of experiments proctolin was tested against injections of combination of proctolin and PhE (protocol 3-3-3) in order to demonstrate the additive effect of $\mathrm{PhE}$ on proctolin-induced stridulation, to reveal some properties of the time course of the PhE effect and to compare the results, received from both 
protocols. The durations of stridulation only increased with the third application of the mixture. It increased from $64 \%(S D=12 \%)$ up to $91 \%(S D=11 \%, P<0,05, n=4)$ and reversed to $64 \%(S D=12 \%)$ in response to the third proctolin pulse following the last PhE pulse (Fig. 3-53, A). Surprisingly, the first and second combined injection of proctolin and $\mathrm{PhE}$, led to a slight decrease of the S Duration from $64 \%$ to $50 \%(S D=28,9 \%)$ but not of C Duration, which remained the same. In other words there was less activity stretched over the same period of time. This was observed in 2 experiments. In the remaining 2 experiments the increase of the duration of singing in response to the combination of proctolin and $\mathrm{PhE}$ already began after the first injection.

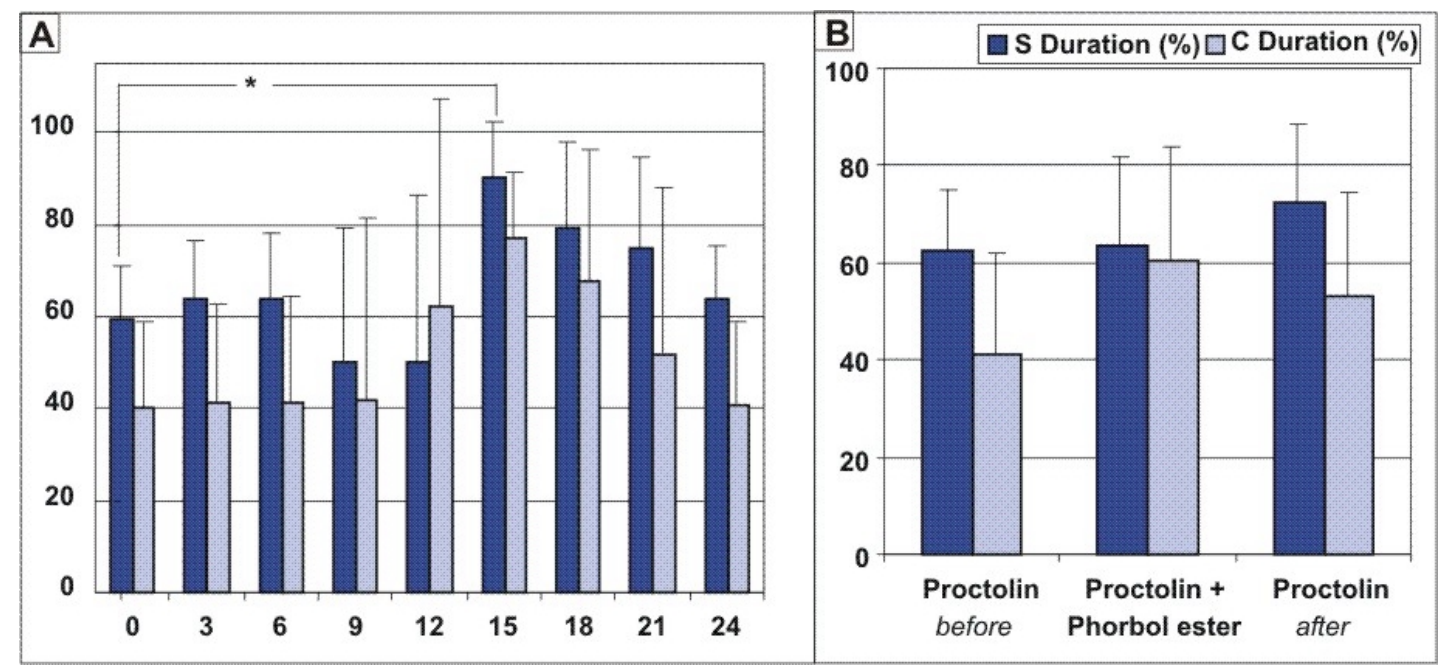

Fig. 3-53 The effect of phorbol ester on proctolin-triggered singing (males, Ch.b., $n=4)$

A. protocol 3-3-3: - 0,3,6 and 18,21,24 puses proctolin; 9,12,15 - pulses proctolin and phorbol ester $\left(C_{M}=1 \mathrm{mM} ; 1: 1\right)$. B. Average values of durations before during and after application of phorbol ester.

All responses $\left(1^{\text {st }}, 2^{\text {nd }}\right.$ and $3^{\text {rd }}$ injections) averaged (Fig. 3-53, B) did not show any significant changes due to co-injection of $\mathrm{PhE}$, since the increased duration after the $3^{\text {rd }}$ injection of the mixture were compensated by the slightly reduced responses to the first two injections. 
In contrast changes in the latencies of the responses were clearly significant in the cumulative evaluation. The latencies were prolonged from initial $28 \%$ $(\mathrm{SD}=9 \%)$ up to $73 \%(\mathrm{SD}=10 \%)$ in response to injection of proctolin and $\mathrm{PhE}$ and reversed to $31 \%$ (SD=13\%) (Fig. 3-54 A, B).
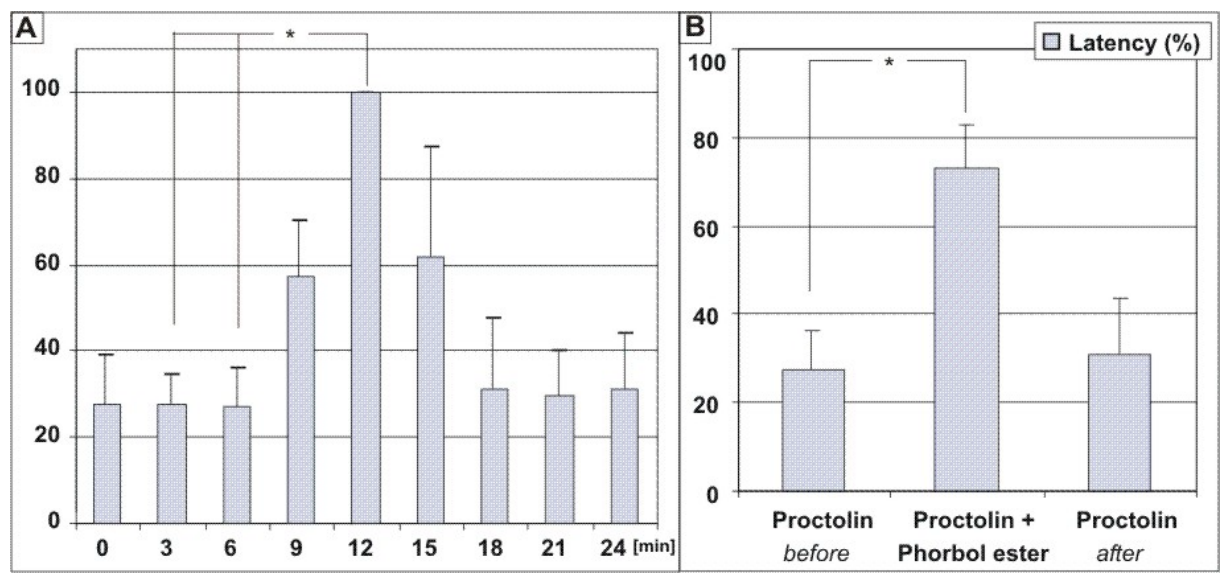

Fig. 3-54 Prolongation of the latencies of proctolin-stimulated singing by co-injection of phorbol ester (males, Ch.b., n=4). A. Protocol 3-3-3: 0,3,6 and 18,21,24 - pulses proctolin; 9,12,15 - pulses proctolin/PhE mixture; B. Averaged latencies before, during and after $\mathrm{PhE}$ co-injection.

This surprising result indicates that might exist a complex interference between the signal transductions, triggered by both stimuli injected simultaneous.

An example of the time course of the responses to proctolin before and after application of $\mathrm{PhE}$ is shown in the following figure (Fig. 3-55, A, B). 

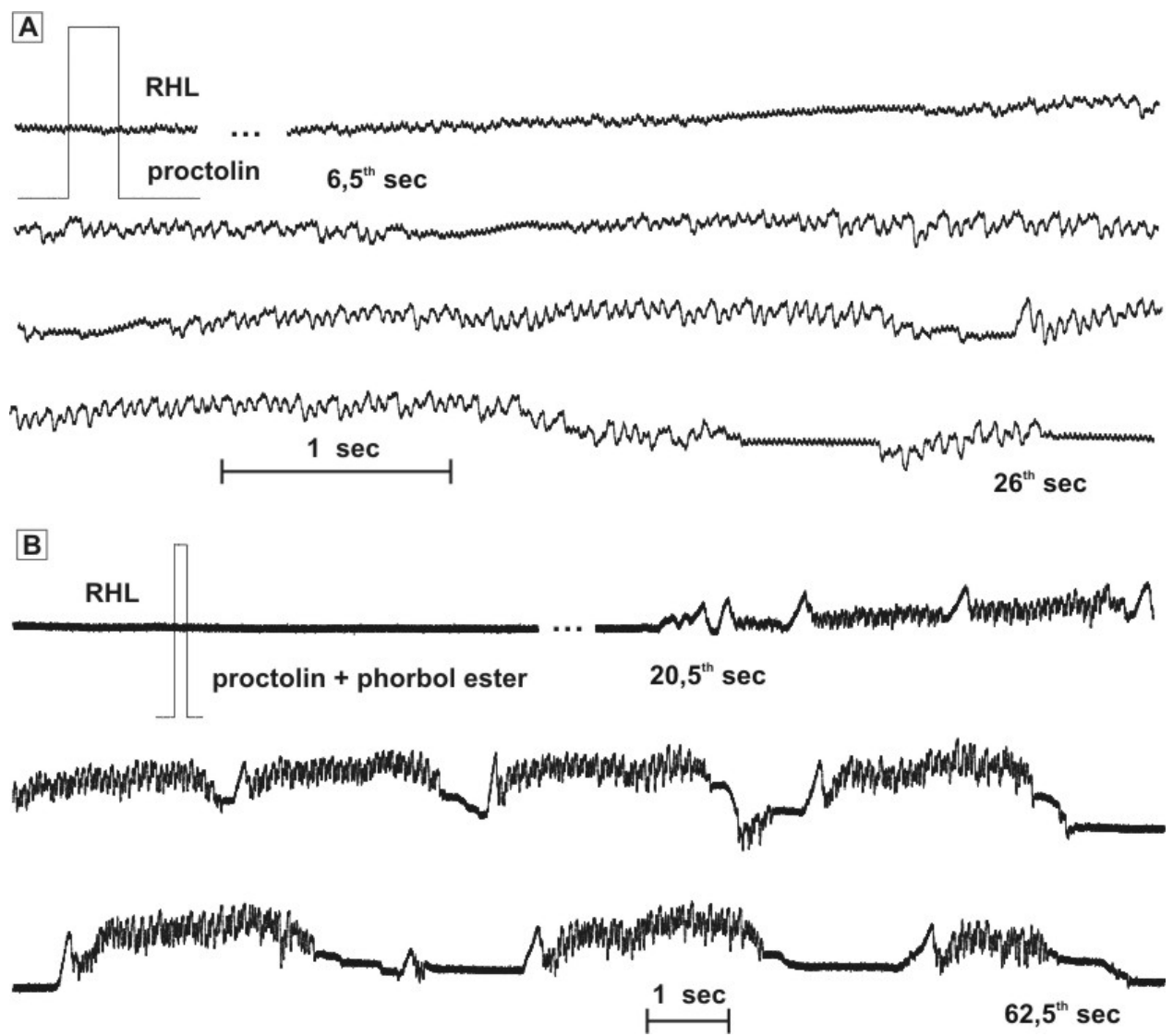

Fig. 3-55 Modulation of proctolin-stimulated singing through co-application or phorbol ester (male, Ch.b.). Both latency and duration were increased.

A. Song sequences induced by proctolin before PhE application; B. Singing elicited by proctolin/PhE mixture. 


\subsubsection{Potassium channels as potential effectors of proctolin receptor}

\section{activation}

It has been demonstrated in various preparations that PRs activation can lead to a decrease of the resting $\mathrm{K}^{+}$conductance, due to an alteration of a particular subliminal current. The cockroach antennal heart responds to proctolin by accelerating the rate of pacemaker depolarizations by more than twofold (Hertel et al., 1985). Presumably, this involves alterations in one of the resting conductances of the membrane.

In early experiments testing the effect of higher concentration of $\mathrm{K}^{+}$ $\left(C_{M}=40 \mathrm{mM}\right)$ in Ringer solution was observed the following observation. In a given spot only once could be induced stridulation (1-2 sequences) by injecting ringer solution, containing $40 \mathrm{mM} \mathrm{K}^{+}$. No response occurred to the following repetitive pulses given at different intervals (30 sec up to $3 \mathrm{~min}$ )(data not shown). Impaling another spot into the brain again only the $1^{\text {st }}$ pulse elicited singing, but not the following pulses. The latter was observed in all stimulations. Surprisingly, applying trial of pulses (generated by pulse generator Master-8, A.M.P.I) with shorter duration (60 msec) and interstimulus interval (20 msec), in order to inject the same amount, stable responses (1-2 sequences) at a given spot were induced not only by the $1^{\text {st }}$ but also by the next stimulations, presented schematic here (right).

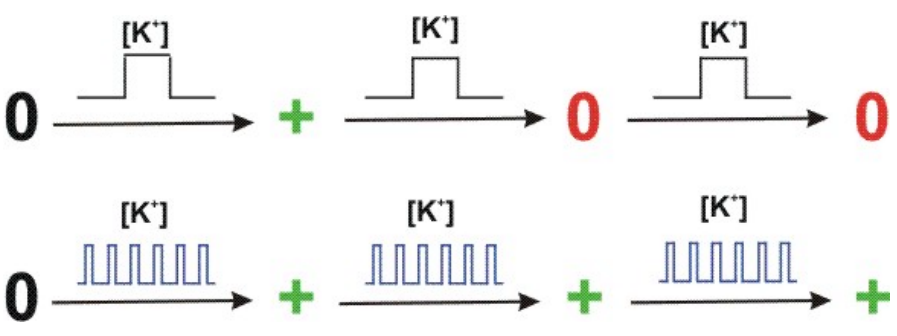

An injection of high concentrated $\mathrm{K}^{+}$solution (up to $60-80 \mathrm{mM}$ ) presumably should not be able to induce depolarization sufficient to synaptic release (Hinton et al., 1998). But it could affect the activity of neurons, which use subliminal $\mathrm{K}^{+}$ channels as intrinsic properties of their firing pattern and so alter their activity.

After these early experiments the concentration of $\mathrm{K}^{+}$in Ringer solution was lowered to $5 \mathrm{mM}$, instead $10 \mathrm{mM}$ in Clement receipt, in order to minimize possible positives. In Ringer solutions, used by other labs, studying insects, the concentration of $\mathrm{K}^{+}$varies between 1,5 and 2mM (Hinton et al., 1997; Wegener et al, 2000). The stronger effect of series of pulses vs. single pulse injected (the same 
amount of the injected substance), on the other hand, indicates that the application mode could be also an object of investigations.

In order to examine a more specific involvement of $\mathrm{K}^{+}$channels in the control of stridulation, unspecific $\mathrm{K}^{+}$channels blocker $\mathrm{Ba}^{2+}\left(\mathrm{C}_{\mathrm{M}}=5 \mathrm{mM}\right)$ was added to the proctolin solution and protocol 3-3-3 was used (Fig. 3-56, A, B).
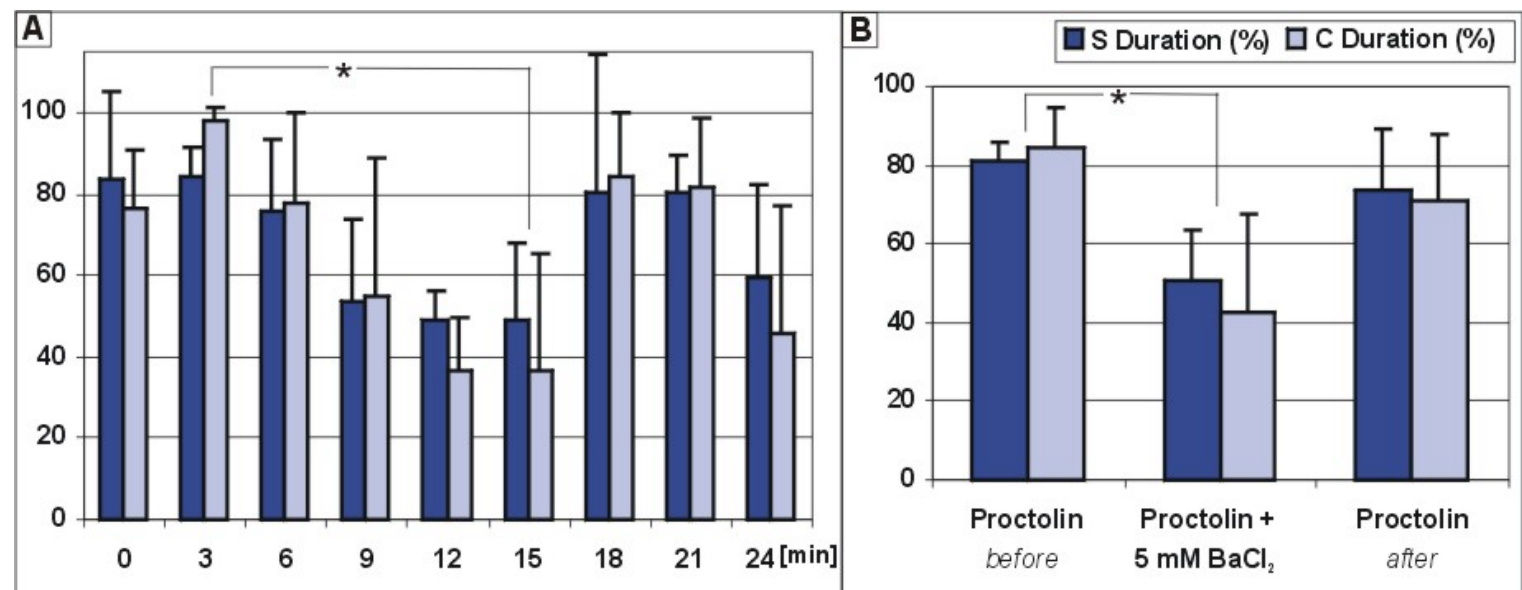

Fig. 3-56 Partial inhibition of proctolin-induced stridulation through the unspecific $\mathrm{K}^{+}$-channel blocker $\mathrm{Ba}^{2+}$ (males Ch.b., $\mathrm{n}=3$ ).

Stridulation, repeatedly induced by proctolin, was partially inhibited by $\mathrm{Ba}^{2+}$, supporting that $\mathrm{K}^{+}$channels influence neuronal activity in cephalic control circuits for stridulation. Whether this is specifically related to proctolin-initiated mechanisms of excitation remains to be demonstrated. 
3.6 Effects of additional transmitter systems on proctolin-stimulated stridulation in male grasshoppers (Ch.b.)

\subsubsection{Dopamine}

It was reported that the actions of dopamine opposed the excitatory effects produced by either proctolin or octopamine in alumina extracts of Limulus midgut tissue. These direct inhibitory actions of dopamine on the Limulus midgut have been mediated by increased levels of cyclic AMP (Groome and Lent, 1992).

Dopamine has been reported to be an important transmitter of higher centers in Drosophila CNS controlling the female sexual receptivity (Neckameyer, 1998). Various immunocytochemical studies (flies, honeybees, locusts) have demonstrated that dopamine is especially contained in the neuropils of central complex (Fig. 3-57) (Homberg, 1994; Roeder, 2002), the brain structure

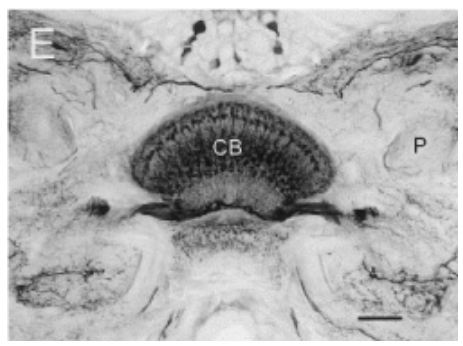

Fig. 3-57 Dopamine-immunostaining in the central body ( $S$. gregaria) (from Homberg, 1994) involved in the control of grasshopper stridulatory behavior. Unpublished results from studies on O.v. have indicated, that dopamine may be capable of stimulating sound production upon injection into the protocerebrum of grasshoppers (Heinrich, not published) .

In order to repeat this preliminary result in Ch.b., it was tried to stimulate stridulation by injections of dopamine in a number of males. In no case could be released stridulation. In contrast, dopamine caused a prolonged $(2-5 \mathrm{~min})$ relaxation of both hind legs, open to the sides, detaching them from the wings and holding them as shown on the photograph (Fig. 3-58). During this dopamine-evoked "relaxation" it was impossible to

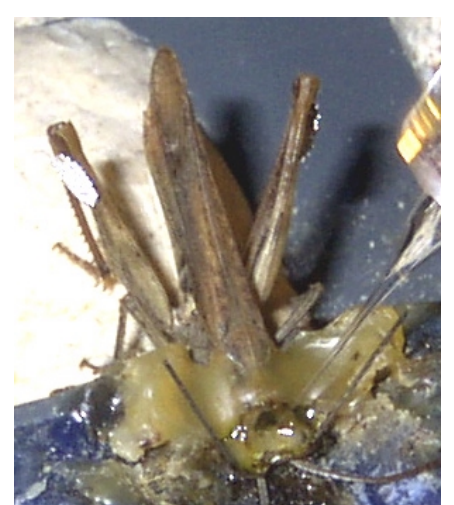

Fig. 3-58 Relaxation of both hindlegs induced by dopamine (male, Ch.b.) initiate stridulation neither by muscarine nor by proctolin (data not shown).

In order to examine potential modulatory effects of dopamine on proctolinstimulated singing in Ch.b. dopamine was co-injected with proctolin, using protocol 3-3-3. 
Dopamine, co-injected with proctolin decreased the duration (S Duration) of proctolin-stimulated stridulation from $72,62 \%$ (SD=13,91\%) down to $25,59 \%$ $(S D=19,92 \%, P<0,01, n=10)$. The responses to the following proctolin pulses reversed to $82,36 \%(S D=11,14 \%)$. Analysis $C$ Stridulation $(P<0,01, n=10)$ gave similar results (Fig. 3-59, B). Interestingly, the response to the next injection of

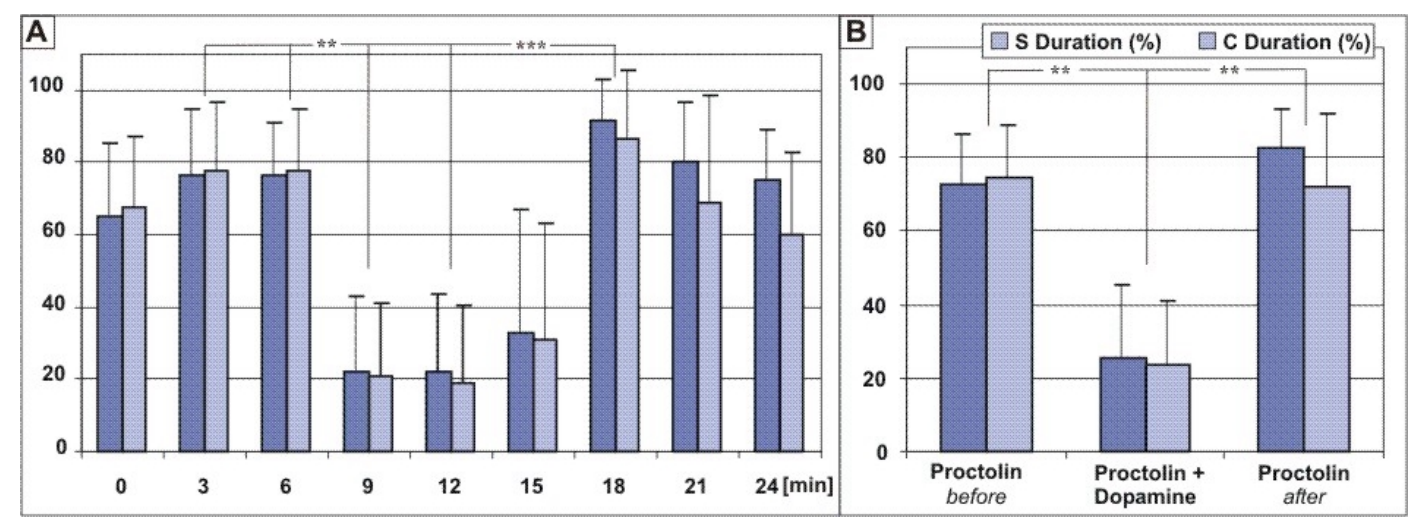

Fig. 3-59 Co-injections of dopamine significantly decreased the durations of proctolinstimulated stridulation (males, Ch.b., $\mathbf{n = 1 0}$ ). A. Prototcol 3-3-3: 0,3,6 and 18,21,24 min responses to pulses proctolin; $9,12,15 \mathrm{~min}$ - responses to proctolin/dopamine mixture $\left(C_{M}=1 \mathrm{mM}\right.$; 1:1). B. Average values of durations before during and after dopamine application $(\mathbf{P}<\mathbf{0 , 0 1})$.

proctolin following the dopamine application lasted longer than the responses preceding it $(91,73 \%, S D=11,18 \%, n=10, P<0,001)$ (Fig. 3-59, $A$ ) although this was not significant. Then the durations following the next proctolin pulses decreased to the initial level. The latter suggests that the dopamine effects may be concentration dependent. This was not further examined.

The latencies of the repetitive proctolin-induced songs were significantly prolonged by co-injections of dopamine (Fig. 3-60, A, B). From the initial level of
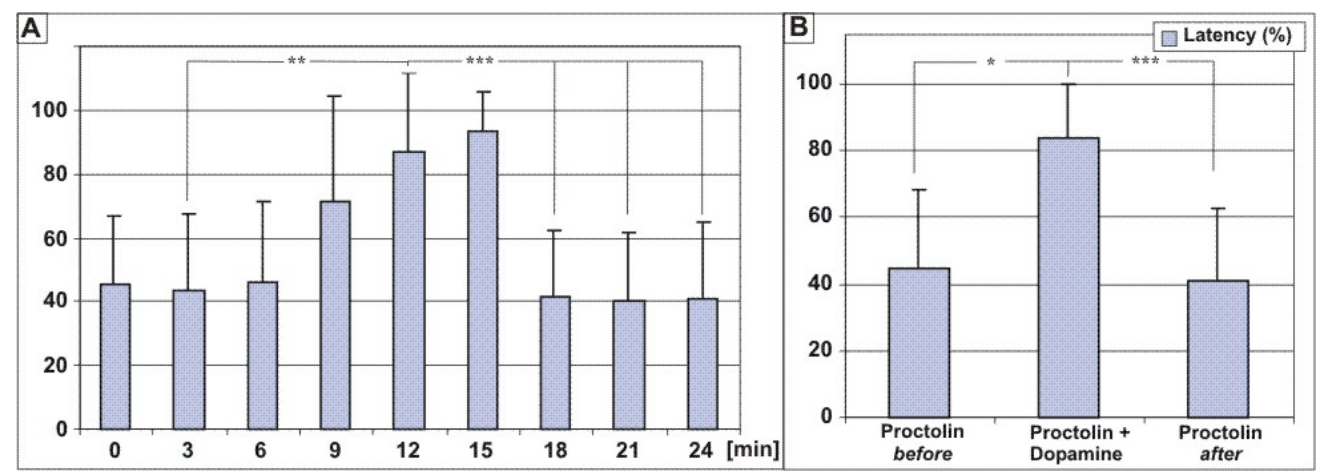

Fig. 3-60 The alteration of the latencies of proctolin-stimulated singing produced by coinjection of dopamine (males, Ch.b., $\mathbf{n = 1 0}$ ). A. Protocol 3-3-3: 0,3,6 and 18,21,24 -responses to proctolin; 9,12,15 - responses to proctolin and dopamine $(1 \mathrm{mM}, 1: 1)$. B. Averaged latencies of the proctolin-stimulated stridulation before $(P<0,05)$, during and after $(P<0,001)$ dopamine co-injection. 
$45 \%(S D=23,21 \%)$ the latencies increased to $83,45 \%(S D=16,17 \%, P<0,05$, $n=10)$ with dopamine and than reversed to $40,81 \%(S D=11,14 \%, P<0,001$, $\mathrm{n}=10$ ) after dopamine application (Fig. 3-60, B).

Typical experiment of this series is presented in Fig. 3-61, A, B, C.
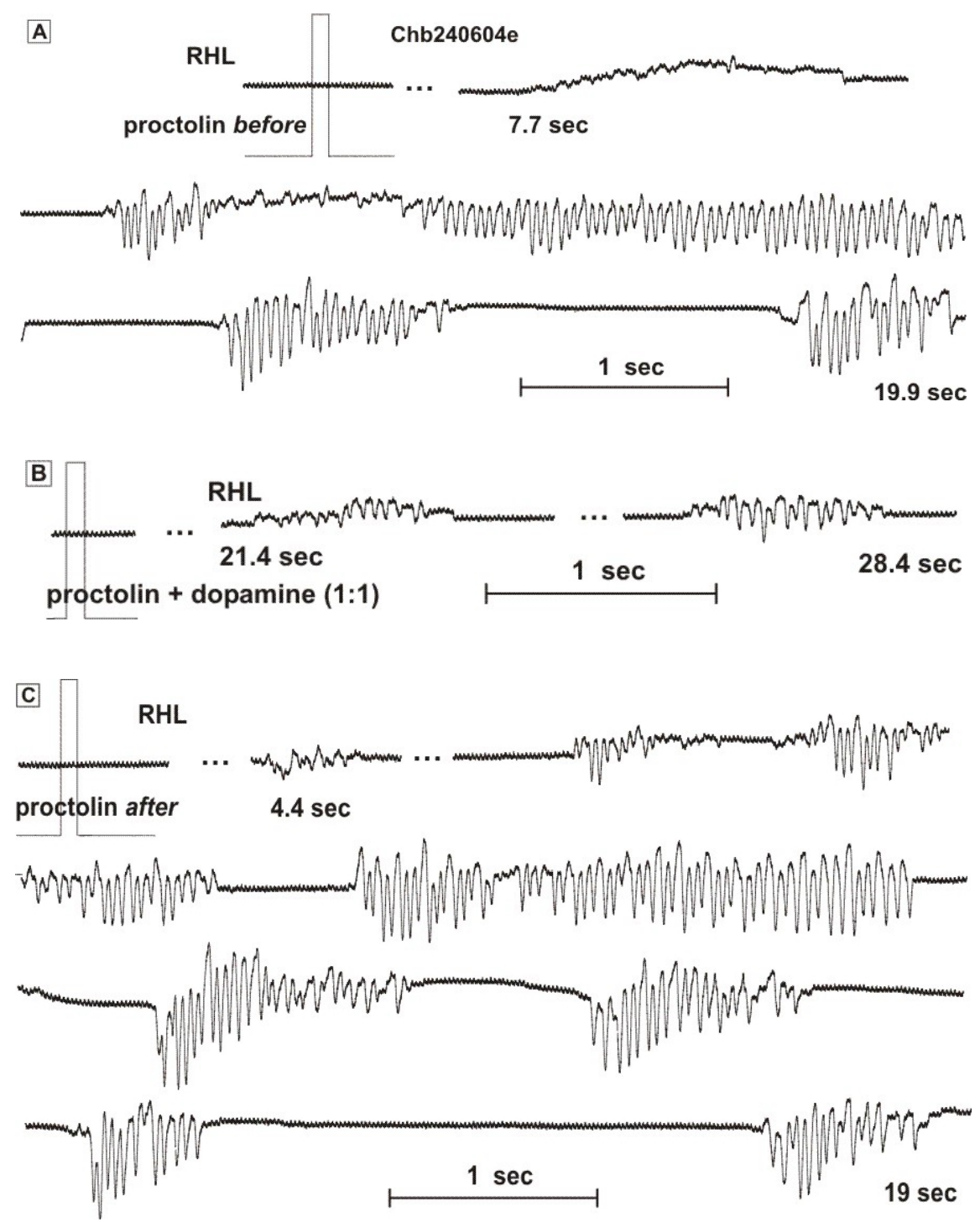

Fig. 3-61 Song sequences, produced by proctolin before (A), with (B) and after (C) dopamine co-injections (male, Ch.b.)

In $5(n=10)$ experiments additional unspecific active hindlegs movements were observed in response to dopamine co-injections. The effect of dopamine on proctolin-stimulated singing might therefore be indirect by triggering another behavioral motor program competing with sound production. An example is shown in Fig. 3-62, B. The stridulatory hindleg movements in response to proctolin and dopamine were of lower amplitude than those elicited by proctolin alone. Similar low-amplitude stridulatory movements were performed in the 
initial phase of the proctolin-induced singing (Fig. 3-62, A, $1^{\text {st }}$ and $2^{\text {nd }}$ line of the signal).
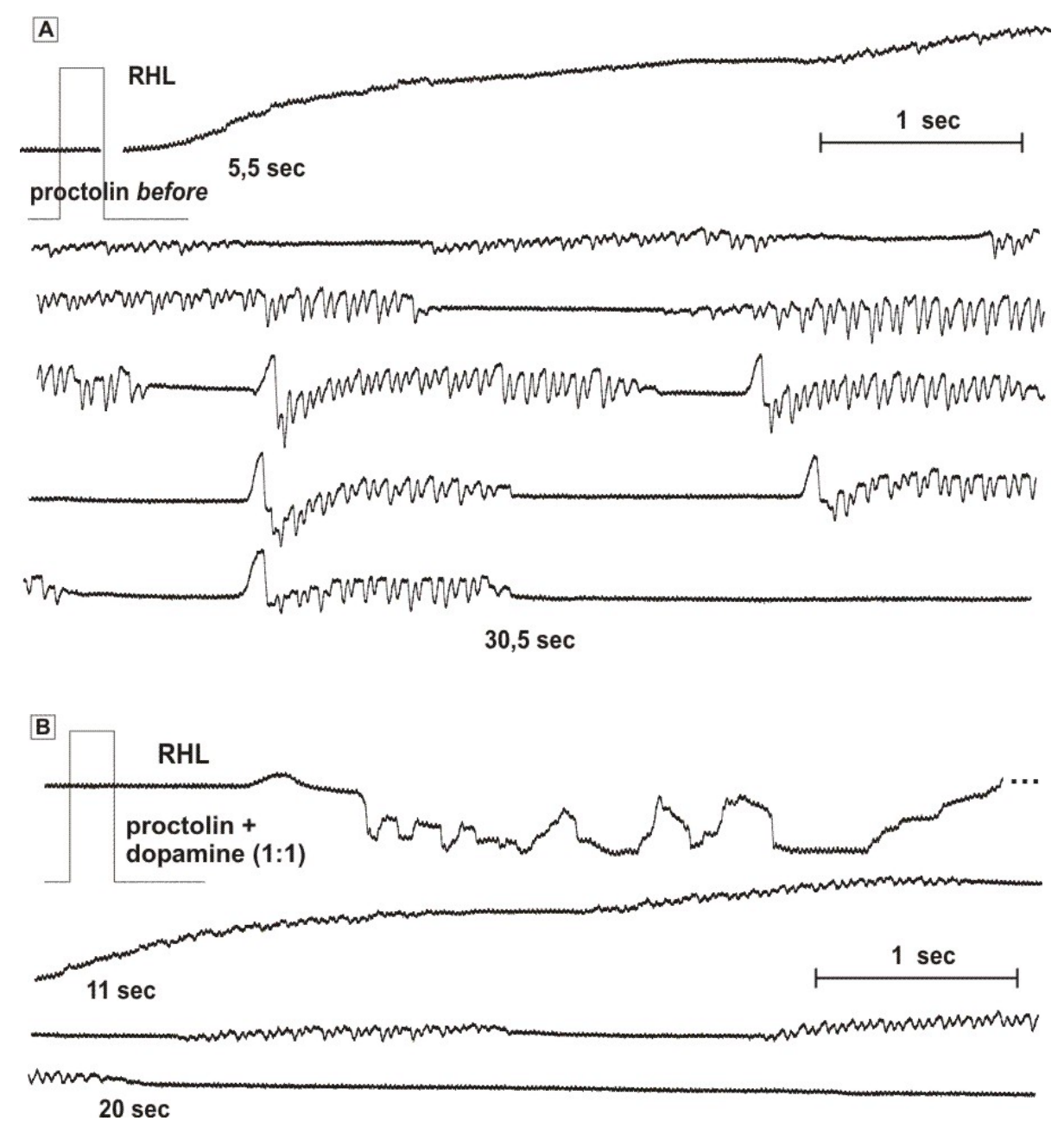

Fig. 3-62 Dopamine, co-injected with proctolin evoked unspecific (struggling) movements and low-amplitude stridulatory movements. A. and C. Song sequences stimulated by proctolin before and after dopamine co-injection (B). Active hindlegs movements followed by song sequences. Similar low-amplitude stridulatory movements were observed in the initial phase of proctolin-produced stridulation before the dopamine application (A. $1^{\text {st }}$ and $2^{\text {nd }}$ line of the signal).

To summarize these results, unlike ACh and proctolin dopamine seems not to be a potent stimulating transmitter in the cephalic song control system of Ch.b., since it is only capable of modulating stridulation stimulated by other neurotransmitters. Dopamine induced a "relaxed" position of the hindlegs, opposite from their oriantation when the animal is about to sing. Co-injection of dopamine with proctolin reduced the stimulated stridulatory activity and the amplitude of the fast stridulatory hindleg movements. A direct interference of dopamine receptors with proctolin-initiated excitation cannot be excluded but most likely, dopamine mediates the above mentioned effects indirectly via 
activating other motor programs that either compete or are related to the performance of stridulation.

\subsubsection{GABA}

It has previously been demonstrated that fast acting transmitters GABA and glycine can rapidly terminate individual song sequences that had started before the injection of the inhibitor in O.v., Ch. molis and Ch.b. (Heinrich et. al., 1998). The inhibitory effect has been of short duration, letting the grasshopper resume stridulatory activity with a new sequence. Within a restricted brain area of the central protocerebrum a termination of song sequences has been achieved by either GABA or glycine, or by both substances at the same brain sites. Different latencies of these inhibitory effects (100-400 msec for GABA and 400-650 msec for glycine) suggested existence of distinct receptors. (Heinrich et al., 1998). It has been reported in this study that in Ch.b. the inhibitory action of GABA and glycine on muscarine-induced stridulation have not differed from those in O.v. and Ch. molis.

To examine the role of GABA-mediated fast and short-lived inhibitory effects Ch.b may not be the best species since its song sequences are relatively short and with variable in duration $\left(1^{\text {st }}\right.$ sequences are longer than the following). Furthermore, the sequences $1^{\text {st }}$ order stimulated by proctolin were shorter 1-3 sec than muscarine-stimulated song sequences. Additional problem was that with the accumulation of the excitation, in contrast to O.v., become shorter. On the other hand, to determinate a control a pulse saline vs. pulse GABA should be injected at the same sites, where proctolin before has elicited singing. That supposes a use of three-barrel microcapillary for proctolin, GABA and saline. Therefore, effects of GABA were only studied in a quantitative manner.

Only in one of five preparations GABA produced inhibitory effect on proctolin-induced stridulation, shown in Fig. 3-63. In all other

proctolin-induced stridulation

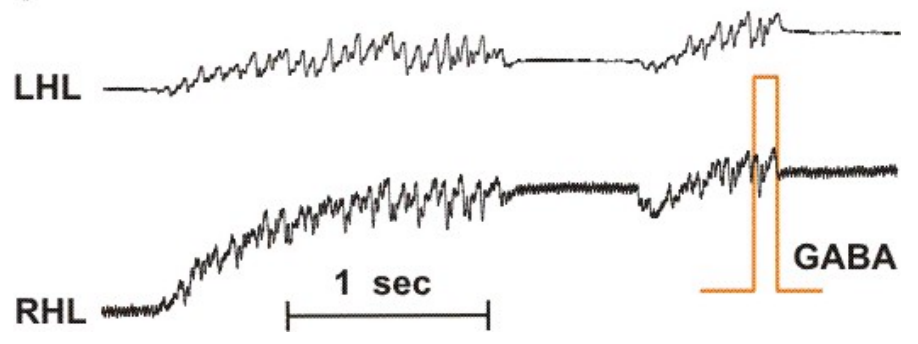

Fig. 3-63 Termination of a song sequence from proctolinstimulated stridulation by GABA (male, Ch.b.) 
preparations GABA had no effect, neither on the duration of a single sequence, nor on the durations of the entire singing.

Because of the difficulties described above no further examination of the prematurely termination of the song sequences $1^{\text {st }}$ order by inhibitory transmitters, serving as ligands of $\mathrm{Cl}^{-}$channels (GABA, glycine, glutamate, histamine) were carried out with Ch.b. males. But the possible modulatory effects of the mentioned inhibitory transmitters co-injected with proctolin on the latency and the durations of the proctolin-stimulated stridulation can be tested.

Ch.b. is suitable to examine slower and longer processes, for instance, the activation of metabotropic receptors, which are gated by these transmitters mGABARs (GABA ${ }_{B}$ Rs), mGluRs, mHisRs.

In two experiments it appeared that GABA co-injected with proctolin slightly enhanced the stridulatory responses to proctolin stimuli (data not shown). This indicated that slow effects of these inhibitory transmitters on proctolin-induced singing in Ch.b. may exist.

Some efforts were also made to study a possible involvement of metabotropic GABA receptors $\left(\mathrm{GABA}_{B} \mathrm{Rs}\right)$. Three preparations were treated with baclofen ( $\beta$-parachlorophenyl GABA), an agonist at vertebrates $G_{A B A_{B}}$ receptors showed no effect on proctolin-induced stridulation (data not shown).

\subsubsection{Glycine}

Glycine co-injected with proctolin had no significant effect on proctolininduced singing. Nevertheless, a slight decrease of the C Duration from $65 \%$ $(S D=23,16 \%)$ down to $48 \%(S D=22,5 \%)$ that susequently reversed to $65 \%$ $(\mathrm{SD}=27,5 \%)$ was observed. In contrast to that, the $S$ Duration increased gradually from $68 \%(S D=20,32 \%)$ to $73 \%(S D=26,5 \%)$ and further to $90 \%$ $(\mathrm{SD}=7,9 \%)$ in response to the proctolin pulses following the co-injections (Fig. 3-64, A, B). A decrease of the durations of the song sequences of $1^{\text {st }}$ order in response to the co-injection of glycine (Fig. 3-64) was observed. Sequences consisting of 2-3 chirps (200-400 msec) could follow the normal ones (2-3 sec) (Fig. 3-65, $A_{1}, A_{2}, A_{3}$ ) in some experiments. Both performed patterns (I and II) of hindleg movements could be clearly distinguished (Fig. 3-65, $A_{4}, A_{5}$ ). The 

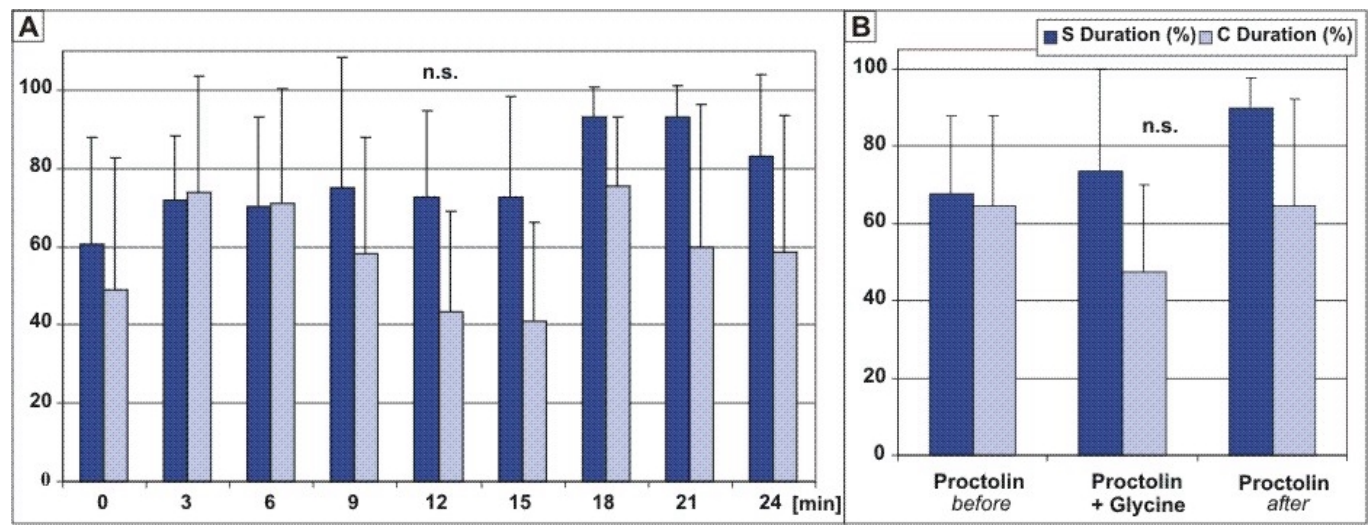

Fig. 3-64 Effects of glycine on proctolin-stimulated stridulation (males, Ch.b., $n=4)$. A. Protocol 3-3-3: 3,6,9 and 18,21,24 - responses to proctolin; 9,12,15 - responses to proctolin and glycine $\left(C_{M}=1 \mathrm{mM} ; 1: 1\right)$. B. Average values of proctolin-produced song before, with and after glycine co-injections.

number of syllables per chirp varied between 4 and 10 within the sequences of $1^{\text {st }}$ order.

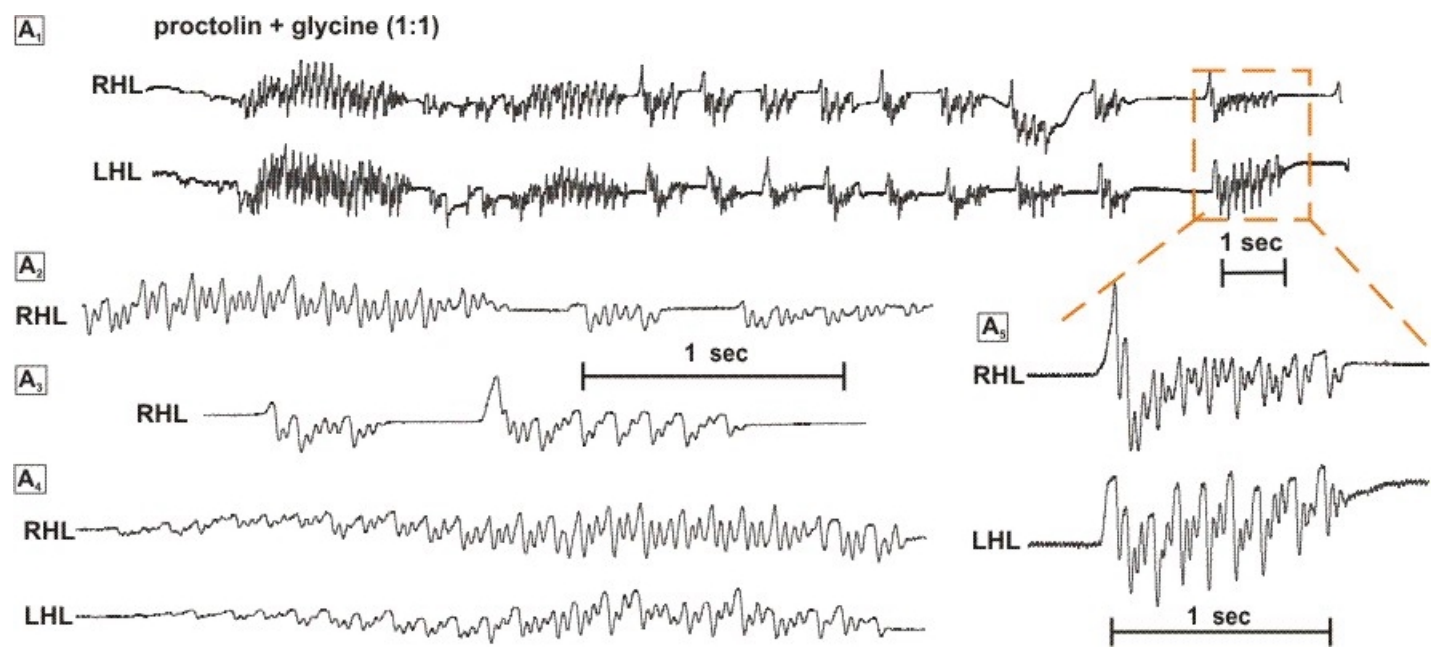

Fig. 3-65 Effects of glycine co-injected on proctolin-stimulated singing (male, Ch.b.). $A_{1} A_{2}, A_{3}$. Very short sequences $1^{\text {st }}$ order (200-500 msec) followed the normal ones. $A_{4}$ and $A_{5}$. Singing pattern I was performed explicit.

Glycine, besides its function as a $\mathrm{Cl}^{-}$-channels ligand, is also known as a ligand of NMDA glutamate ionotropic receptors (iGluRs) in vertebrates, which are permeable to monovalent cations and with different permeabilities to $\mathrm{Ca}^{2+}$.

Therefore, the next step was to look for a role of glutamate in the modulation of proctolin-maintained singing in Ch.b. 


\subsubsection{Glutamate}

The distribution of glutamate throughout insect brain has been reported in different studies (Fig. 366) (Homberg, 1994; Schürmann, 1997; 2001).

Glutamate is known as a ligand of both ionotropic (iGluRs) and metabotropic receptors (mGluRs). iGluRs include $\mathrm{Cl}^{-}$-channels and cation channels - AMPA, Kainate and NMDA receptors. The latter are highly permeable to $\mathrm{Ca}^{2+}$ and have glutamate and glycine activation sites. Thus, they are involved in triggering of fast synaptic transmission. The mGluRs, on the other hand, are coupled to second messenger cascades. Activation

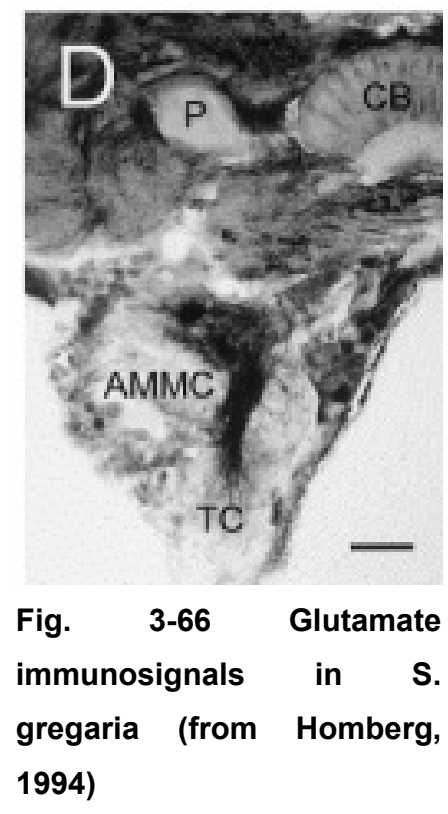
of PLC, inhibition of $A C$, modulation of membrane $\mathrm{K}^{+}$currents and other subliminal conductances have been described (Hammond, 2001).

In O.v. glutamate has been injected into brain regions corresponding to the sites, where acetylcholine injections have elicit stridulation, but none significant leg movements have been stimulated (Ocker et al., 1995).

A first series of experiments tested the effect of glutamate $(0,1 \mathrm{mM})$ coinjected with proctolin on the proctolin-produced singing in males Ch.b. In 3 animals was observed slightly, but not significant increase of the durations in response to co-injected glutamate $\left(\mathrm{C}_{\mathrm{M}}=0,1 \mathrm{mM}\right)$ from $66 \%(\mathrm{SD}=17,7 \%)$ up to $84 \%(\mathrm{SD}=8,8 \%, \mathrm{n}=3, \mathrm{P}>0,05)$ (Fig. 3-67, B).
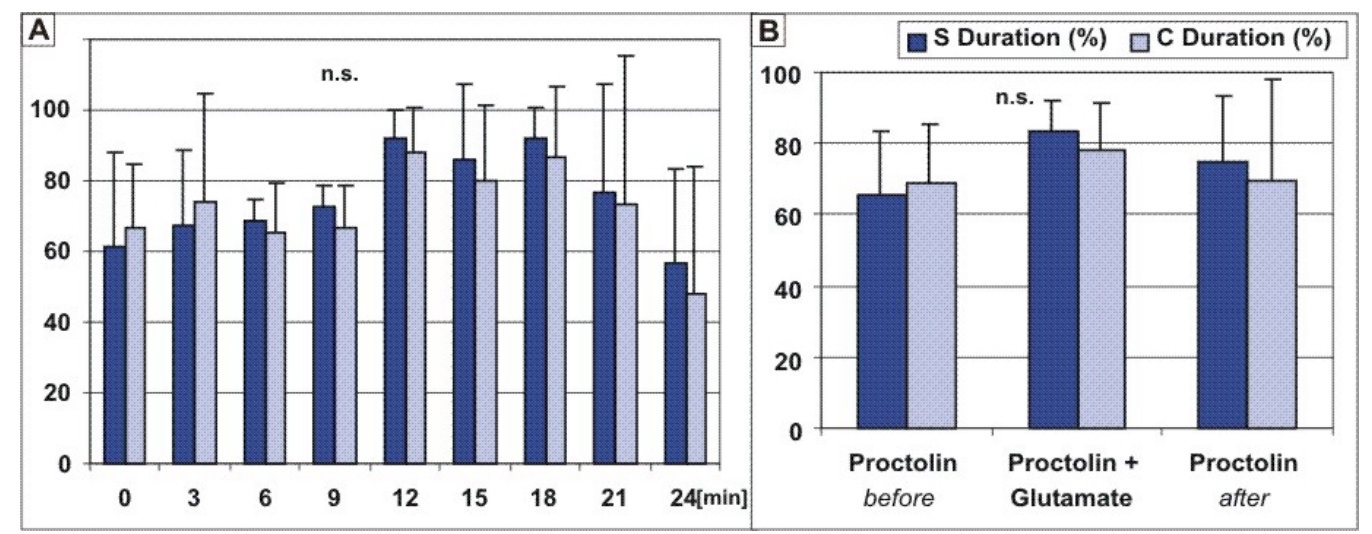

Fig. 3-67 Slightly increase of the durations of proctolin-evoked singing by co-injection of glutamate (1mM; 1:1)(males, Ch.b., n=3). A. Protocol 3-3-3: 0,3,6 and 18,21,24 - responses to proctolin; 9,12,15 - responses to proctolin and glutamate. B. Average values of the durations before, with and after glutamate application. 
In other experiments $(n=5)$, after co-injection of higher concentrated glutamate $(1 \mathrm{mM})$ the increase in the duration of singing was beyond any expectations and resulted in more than $300 \mathrm{sec}$. These data were not included in the evaluations for Fig. 3-67 In one of these experiments additional qualitative changes of proctolin-stimulated stridulation appeared. Before glutamate application the proctolin-induced singing was short and started after a short latency in response to all 3 proctolin stimuli (3 min interval in between) (Fig. 3$68, A)$.

A dramatically prolonged singing activity ( $>10 \mathrm{~min}$ ) was induced by the proctolin injection following three glutamate co-injections (Fig. 3-68, B), similar to the effect of eserine on both proctolin- and muscarine-stimulated singing (section 3.4.6.2, Fig. 3-33, C; Fig. 3-34, B).

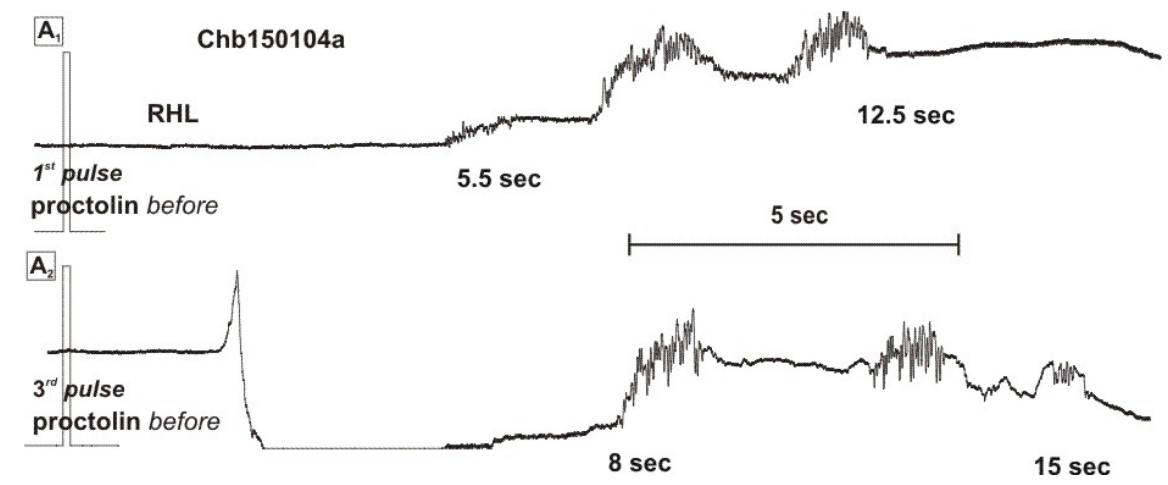

Fig. 3-68 A. Proctolin responses before application of glutamate - after the $1^{\text {st }}$ pulse $\left(A_{1}\right)$ and after the $3^{\text {rd }}$ pulse $\left(A_{2}\right)$ of proctolin, using protocol 3-3-3 (male, Ch.b.).

Interestingly, two rhythms of singing parameters were observed following this stimulation. Long period oscillations consisted long response ( $\sim 30 \mathrm{sec})$ after a long latency $(\sim 6 \mathrm{sec})$, and short period oscillations respectively - short latency ( $30 \mathrm{sec})$ and durations ( 12 sec). Interestingly, both latencies 30 and $60 \mathrm{sec}$ of short and long responses corresponded to the desensitization period of proctolin-stimulated stridulation by repetitive pulses (section 3.2.5.2).

Following this correlation [either long latency/long duration (LL-LD) or short latency/short duration (SL-SD) (Fig. 3-68, B)] it was possible within this stimulation to predict the duration of the next performed series song sequences just looking at the latency. 


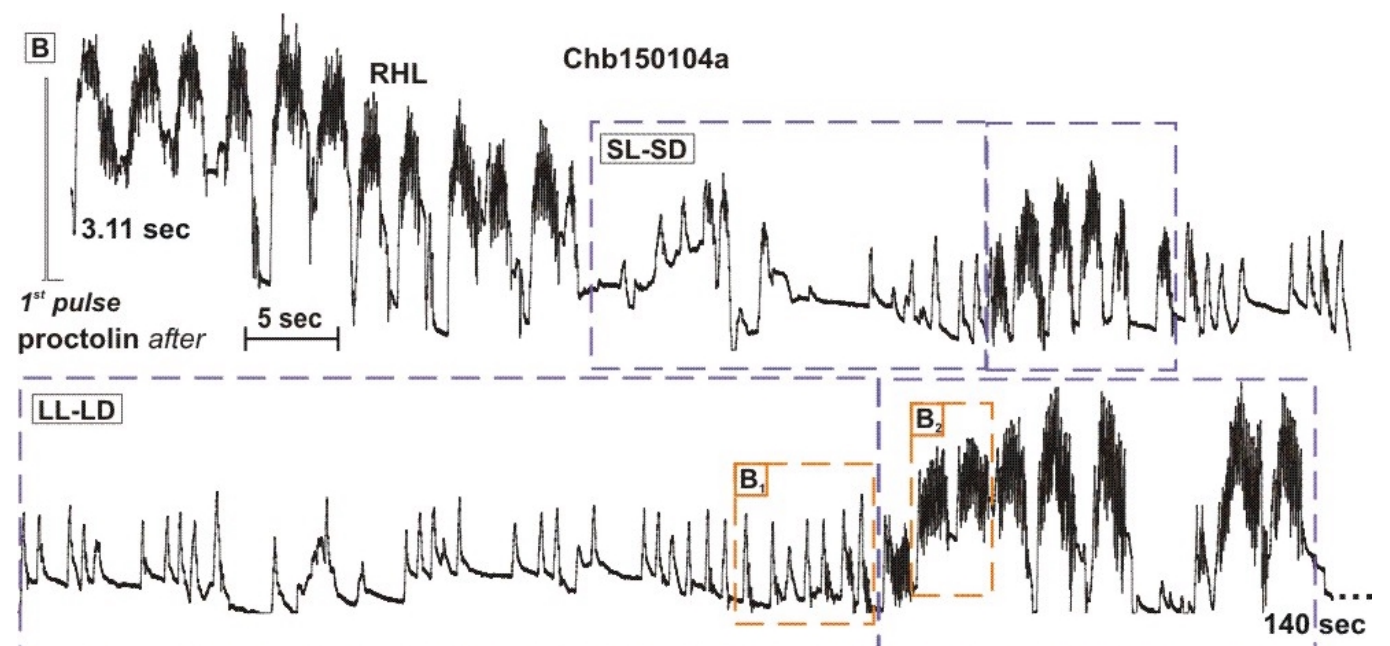

Fig. 3-68 B. Prolonged singing stimulated by folloing the glutamate co-injection proctolin pulse (see the text).

In between the song sequences occurred regular slow up- and downhindleg movements, which initiate short stridulatory movements, but immediately trailed away (Fig. 3-68, $\mathrm{B}_{1}$ ). These movements were similar to the larger-amplitude movements that normally initiate a $1^{\text {st }}$ order song sequences as seen in Fig. 3-68, $B_{1}$. But since the proctolin receptors may be desensitized no stridulatory movements after the initial movement could be performed. Therefore, it seems that this initial movements probably are induced by glutamateinitiated mechanisms.

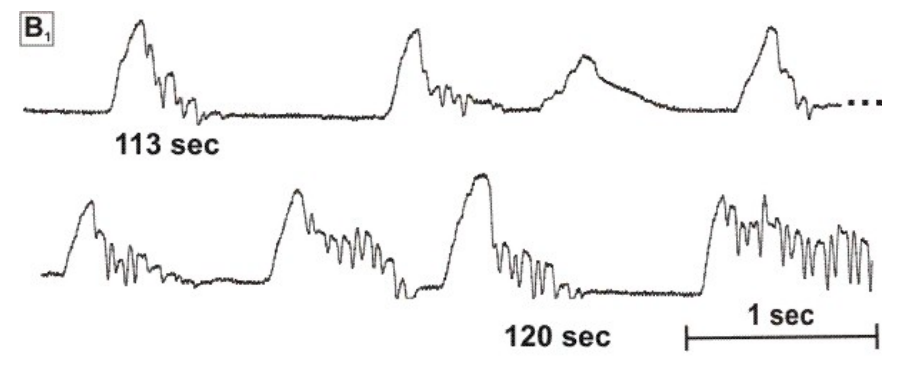

$B_{2}$

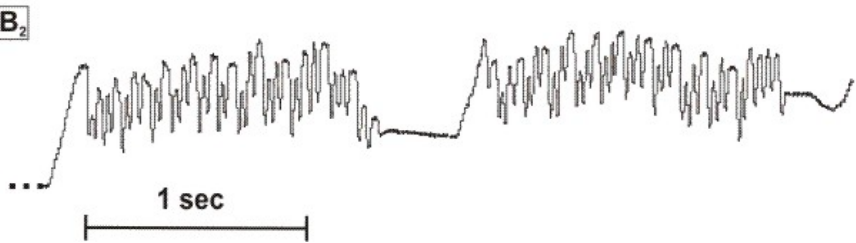

Fig. 3-68 $\mathbf{B}_{1}$. Short song sequences including single or several chirps $\left(B_{1}\right)$, beginning by slow up-movement with high amplitude. These quasi-sequences preceded the normal ones in this stimulation $\left(B_{2}\right)$

Entire singing activity in this experiment is plotted in Fig. 3-68, $\mathrm{B}_{3}$. Both rhythms alternated subsequently and with the decreasing of the excitation remained only the shorter one. 


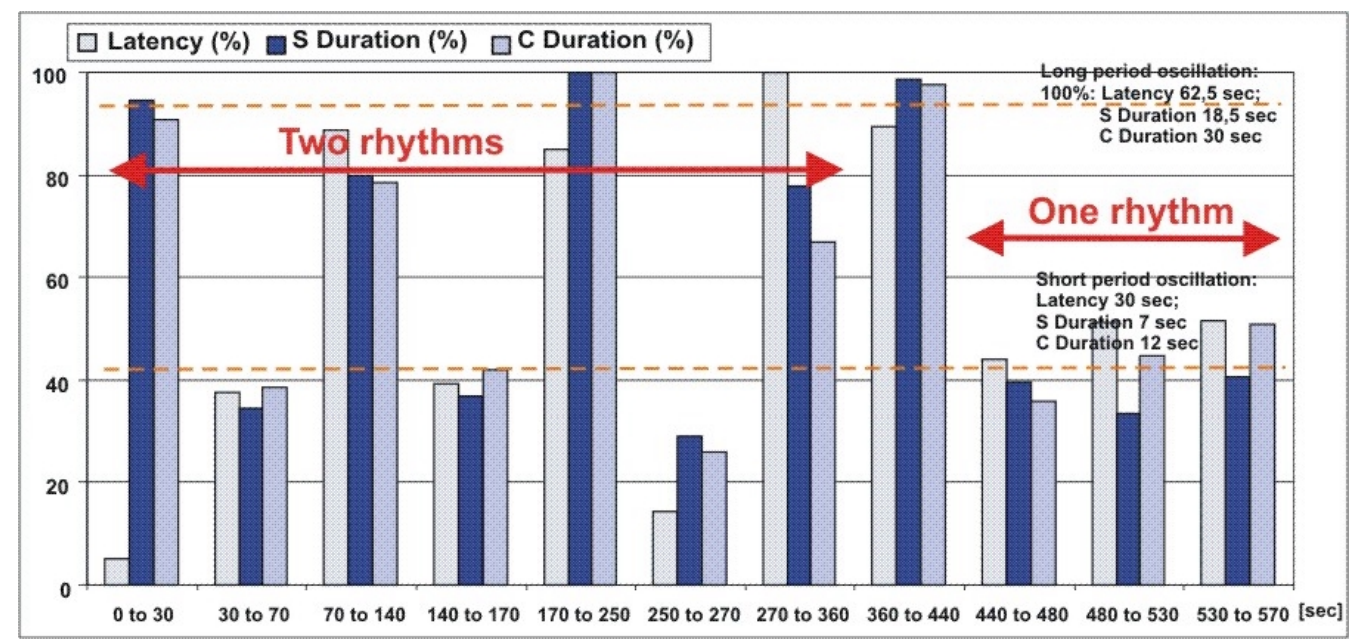

Fig. 3-68 $B_{3}$. Prolonged singing in response to the next proctolin pulse after repetitive glutamate co-injections (male, Ch.b.) Two rhythms of the singing parameters were observed - long [long latency $(\sim 60 \mathrm{sec})$ and durations $(\sim 20 ; 30 \mathrm{sec})]$ and short [short latency (30 sec) and durations $(7 ; 12 \mathrm{sec})]$

In addition to or dependent on the desensitization of the proctolin receptors it can be suggested rhythmic "response readiness" of the brain system, controlling the sound production in Ch.b. Furthermore, the period of the oscillations might increase/decrease stepwise and not linear. In other words the total basal excitation of the stridulatory control system in the brain of Ch.b. may oscillate intrinsic.

Another observation in this stimulation was an alteration of the number of the syllables within a single chirp (Fig. 3-68, C). These alterations were dependent on the duration of the sequences $1^{\text {st }}$ order, the amplitude of the hindlegs movement, respectively the level of excitation. Short and weak song sequences consisted from movements without any chirp structure to 4 syllables chirps. The louder sequences were composed of 4-6 syllables per chirp and the most powerful sequences consisted 8-10 even 14 syllables per chirp (Fig. 3-68

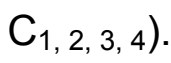



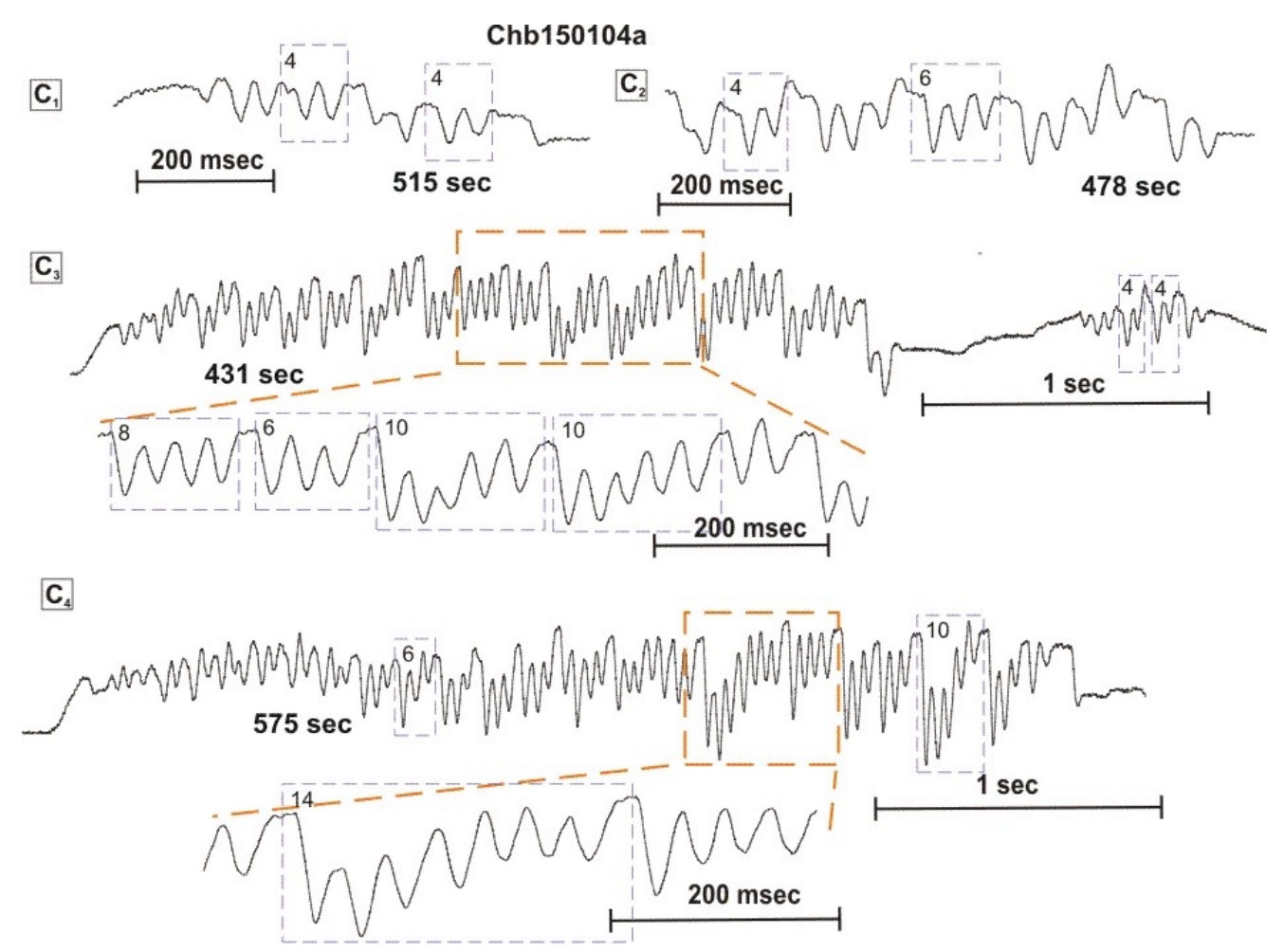

Fig. 3-68, C. Alterations of the number of the syllables within the chirps, dependent on the level of excitation (the duration of the sequences $1^{\text {st }}$ order and the amplitude of the stridulatory movements): $\mathrm{C}_{1} .4$ syllable chirps; $\mathrm{C} 2.4$ and 6 syllable chirps; $\mathrm{C}_{3} .6$ to 10 syllables per chirp, but the following short sequence, consists 4 syllables per chirp; $\mathrm{C}_{4}$. even 14 syllables per chirp.

In other experiments in response to the combination of proctolin and glutamate, precopulatory movements (PMs) ("tick"-movements) were performed either alone (Fig. 3-69, $A_{1}, A_{2}$ ) or embedded into the normal song sequence (Fig. 3-69, $A_{3}$ ). The latter differed from muscarine-induced precopulatory movements in Ch.b. (Wenzel et al., 2000), which have always performed after a normal song sequences at a high level of excitation. Interestingly, the movement patterns could rapidly switch between PMs and stridulatory movements (Fig. 3-69, $A_{2}$ ). 


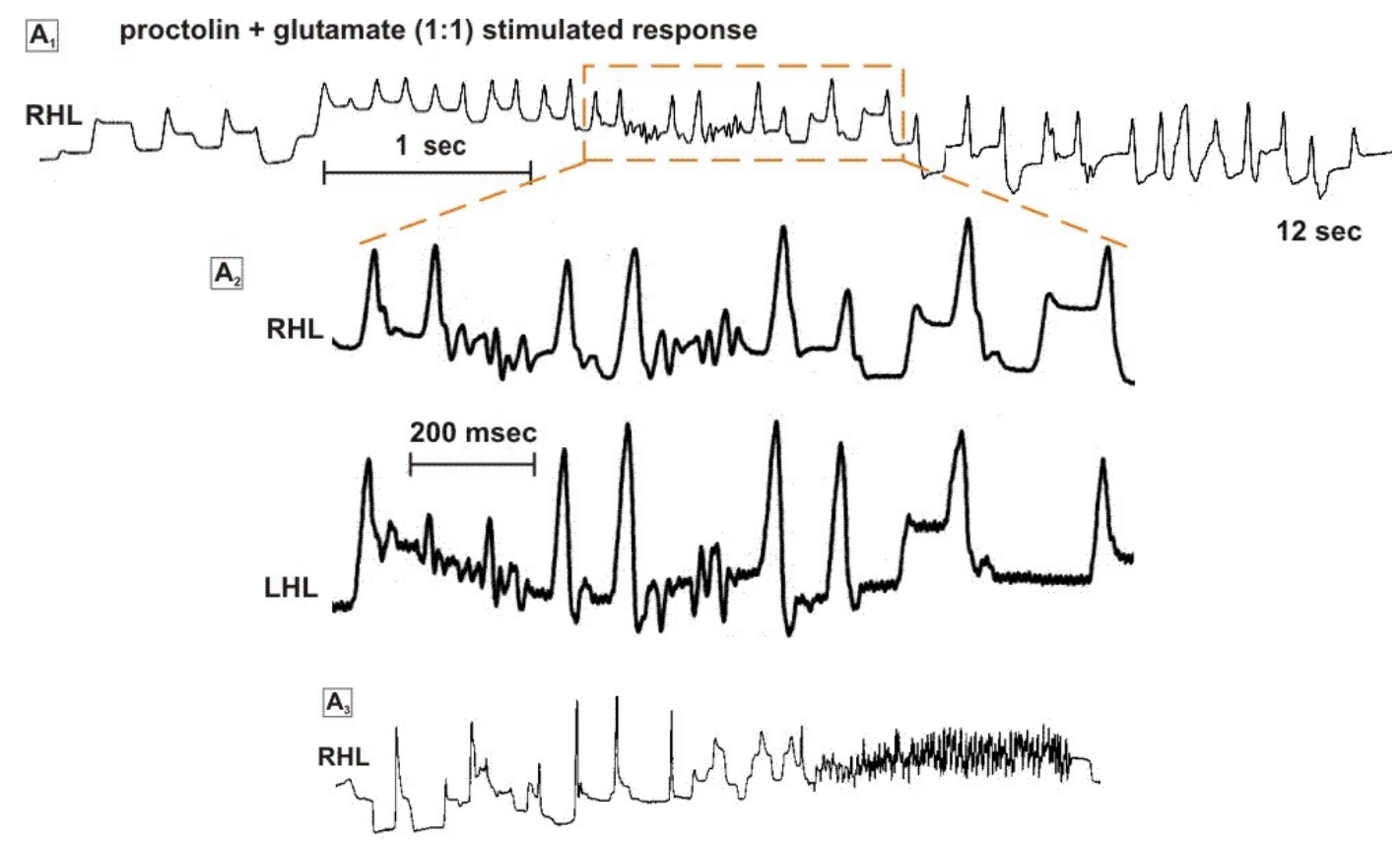

Fig. 3-69 $A_{1}$. Precopulatory movements induced by co-injection of proctolin and glutamate (male, Ch.b.). A . Single specific stridulatory movements occurred in between the "ticks"; Phasic and pattern differences between the movements performed by both HLs were observed. $A_{3}$. Precopulatory movements preceding normal song sequence

The appearance of pharmacologically induced single stridulatory movements and their modulation and variations could be an indication that the fast movements could be also generated and modulated by the brain in Ch.b.

The additional excitation and modulation elicited by glutamate to proctolintriggered singing arouse the following questions: Can glutamate induce alone stridulatory movements in Ch.b.? Which type glutamate receptors (iGluRs, mGluRs or $\mathrm{Glu}-\mathrm{Cl}^{-}$channels) may mediate the glutamate-induced effects? 
When glutamate was injected into protocerebrum, where proctolin reliably induced singing in Ch.b., only precopulatory movements of the hindlegs were elicited, without any regular stridulatory movements in 3 from 5 animals (Fig. 3$70, A_{1}, A_{2}$ ). Appearance of this effect was robust (could be repeated) and

A glutamate $2 \mathrm{mM}$

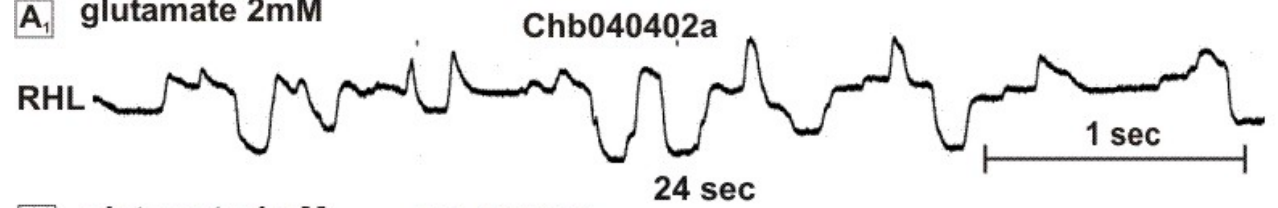

$A_{2}$ glutamate $1 \mathrm{mM}$

$24 \mathrm{sec}$

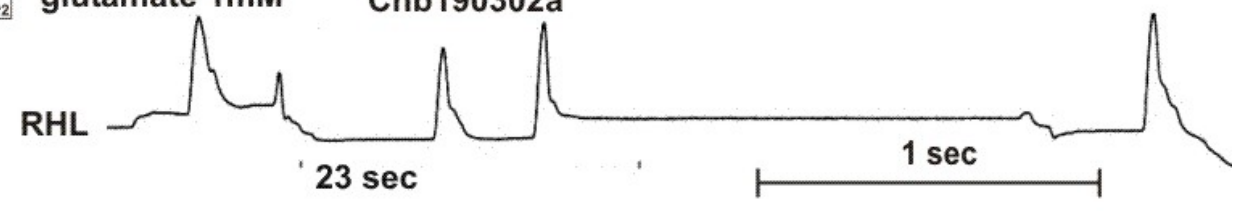

$A_{3}$ glutamate $(1 \mathrm{mM})+$ proctolin $(0,3 \mathrm{mM}) \quad$ Chb120302a

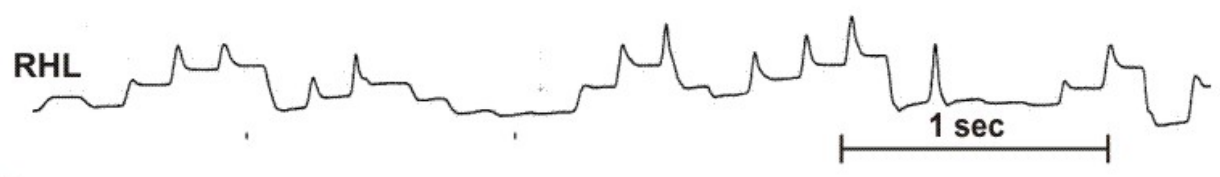

$\mathrm{A}_{4}$ glutamate $(1 \mathrm{mM})+$ proctolin $(1 \mathrm{mM})$

Chb190302b
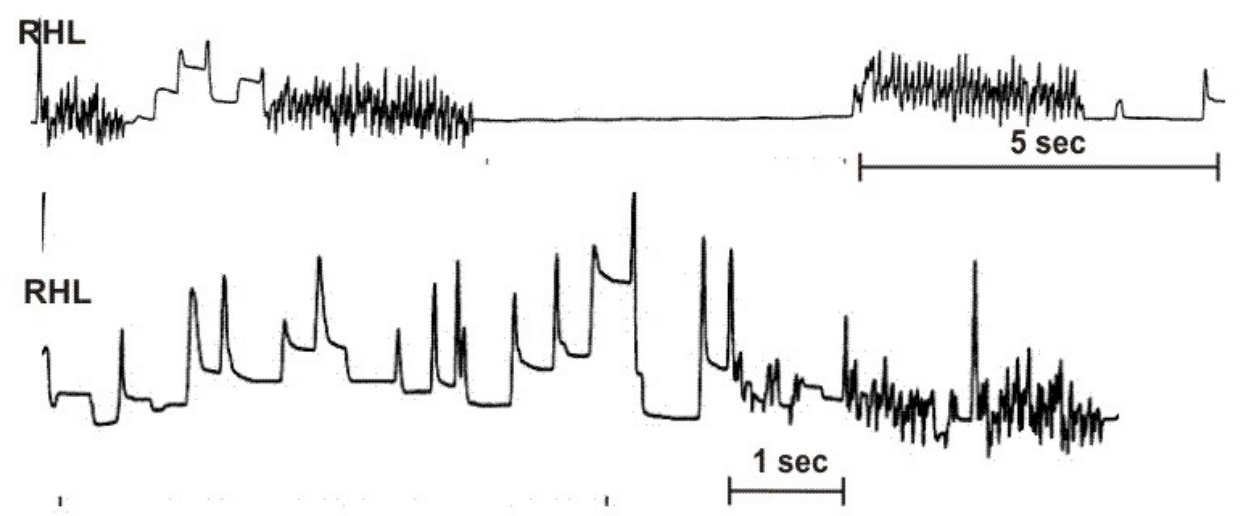

$A_{5}$ glutamate $(1 \mathrm{mM})+$ proctolin $(1 \mathrm{mM})$ Chb040402c

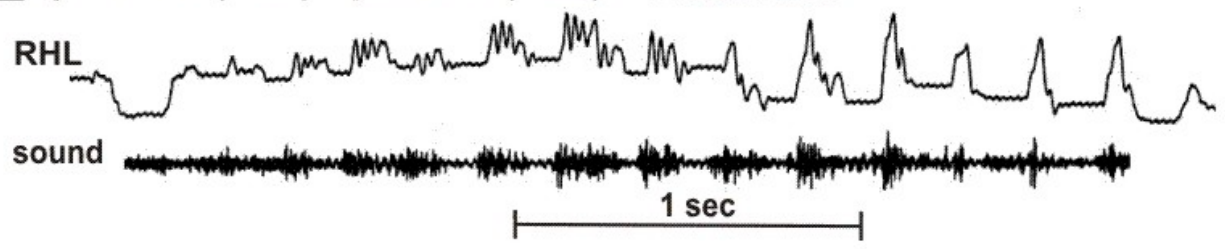

$A_{6}$ glutamate $(1 \mathrm{mM})+$ proctolin $(1 \mathrm{mM})$ Chb040402c

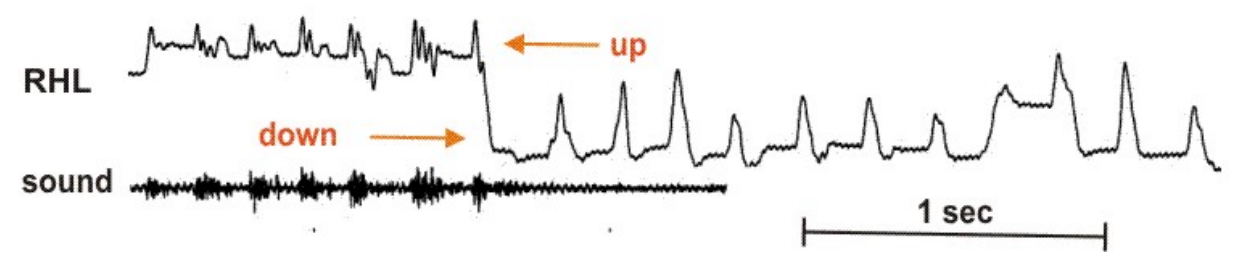

Fig. 3-70 A. Summary of glutamate effects on proctolin-induced stridulation. Evidences of the role of co-transmission in the control of singing behavior (males, Ch.b.) (see the text) 
restricted. The number of PMs, initiated by glutamate, increased when proctolin was co-injected (Fig. 3-70, $A_{3}, A_{4}$ ).

In some preparations glutamate co-injected to proctolin produced very short sequences, which were similar to sridulatory movements, corresponding to a particular chirps (Fig. 3-70, $A_{5}, A_{6}$ ), similar to glycine- (Fig. 3-65) and muscarine-induced ones (Fig. 3-25). The sound produced by the PMs in response to co-injection of glutamate and proctolin was in some preparations very loud (Fig. 3-70, $A_{5}, A_{6}$ ). The movement pattern of the hindlegs in other experiments was somehow dependent on their position - up or down (Fig. 3-70, $\left.A_{6}\right)$.

To explore glutamates potential capability to stimulate stridulation in Ch.b., 3,5-dihydroxyphenylglycine (DHPG), a selective agonist at metabotropic glutamate receptors (mGluR1 and mGluR5), coupled to PLC activation (Hamond, 2001), was injected into the control areas within the brain.

DHPG did initiate singing behavior without any additional excitatory substance (muscarine, proctolin, picrotoxin) (Fig. 3-71, B). The response was robust. The movements within song sequences were of the species-typical patterns though minor variations could occur in response to both DHPG alone (Fig. 3-71, $\mathrm{B}_{1}$ ) or co-injected with proctolin (Fig. 3-71, $\mathrm{B}_{3}$ ). DHPG induced in one case irregular alternations of the singing pattern I and II within a single sequence (Fig. 3-71, $\mathrm{B}_{2}$ ).

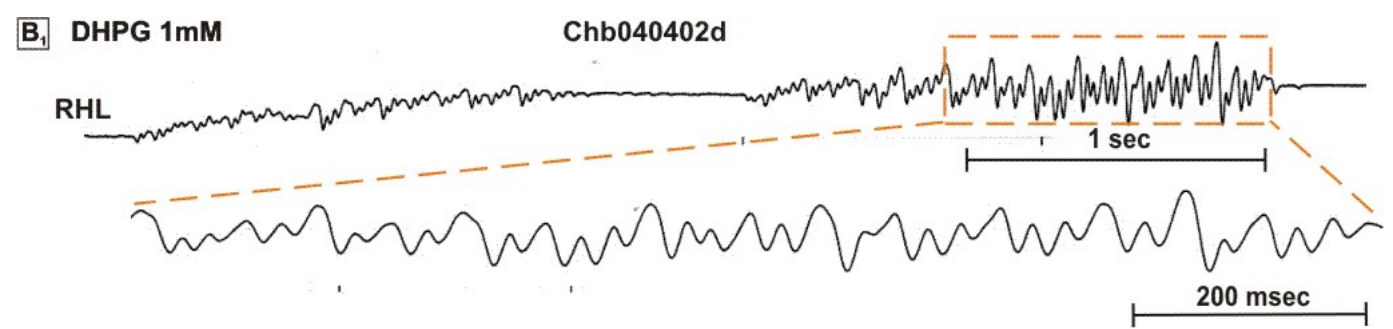

$B_{2}$ DHPG $(1 \mathrm{mM})+$ proctolin $(1 \mathrm{mM}) \quad$ Chb190302d

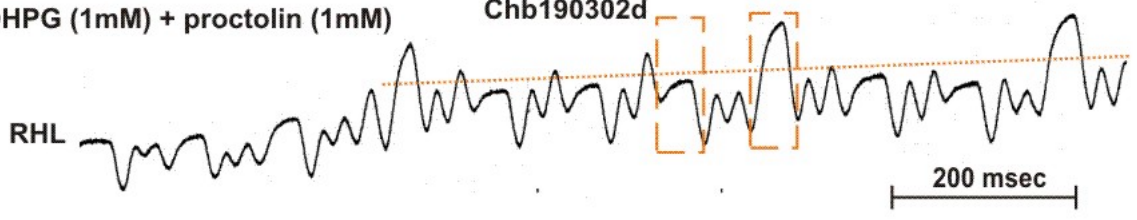

B B $_{3}$ DHPG $(1 \mathrm{mM})+$ proctolin $(1 \mathrm{mM}) \quad$ Chb190302c

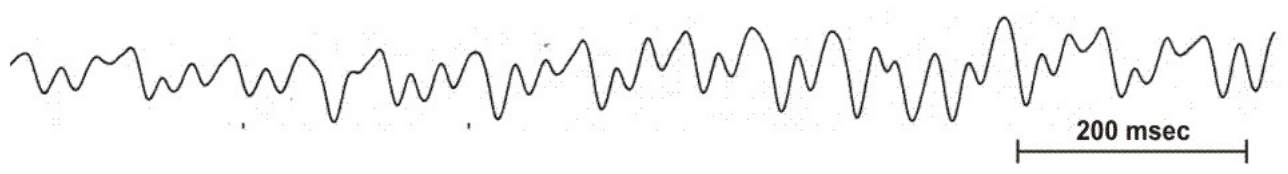

Fig. 3-71 B. mGluR1 and mGluR5 selective agonist, DHPG, triggered stridulation injected alone. (see the text) 
Interestingly, no precopulatory movements were performed in response to DHPG, injected alone or together with proctolin. There was a clear difference between glutamate and DHPG effects, indicating activation of other glutamate receptors than mGluRs $(1,5)$. Fast neutralization of injected glutamate may also interfere with glutamate initiating stridulation, which in contrast could be obtained with DHPG.

These results can be seen as evidence that co-release of glutamate, ACh (see above) and/or other transmitters with proctolin may modulate patterns of stidulation under particular physiological conditions.

Another indication of modulation through co-transmission was found in the switch between different motor programs, namely regular stridulation and wing movements in 4 preparations.

In one experiment both were induced through injection of glutamate during ongoing proctolin-stimulated stridulation. This experiment will be described in details.

In order to examine faster effects on proctolin-induced stridulation glutamate was injected during ongoing singing. The stimulation protocol included 2 injections proctolin with interstimulus interval $12 \mathrm{sec}$ as a control vs. proctolin and glutamate with the same interstimulus interval as a test. In the control stimulation proctolin impulse during proctolin-stimulated singing did not produce any effects (Fig. 3-72, A). Surprisingly in response to the glutamate injection $7 \mathrm{sec}$ from the onset of the stridulation, at the end of the song sequences (26 sec after the glutamate application) wing flapings were induced (Fig. 3-72, B). 
The latter was accompanied by initial "tick" hindleg movements similar to these induced by glutamate (see above) and those that initiate a song sequences of $1^{\text {st }}$ order. The reason for losing the signal in the end was due to covering of the reflecting discs positioned on the hindlegs by the wings.

A

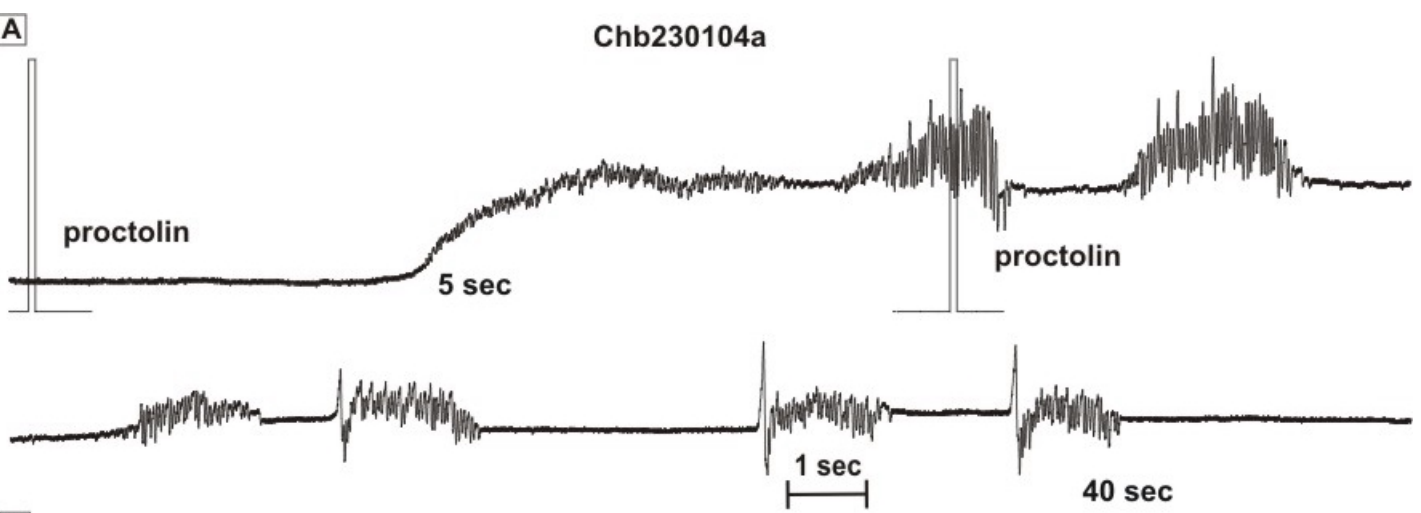

B
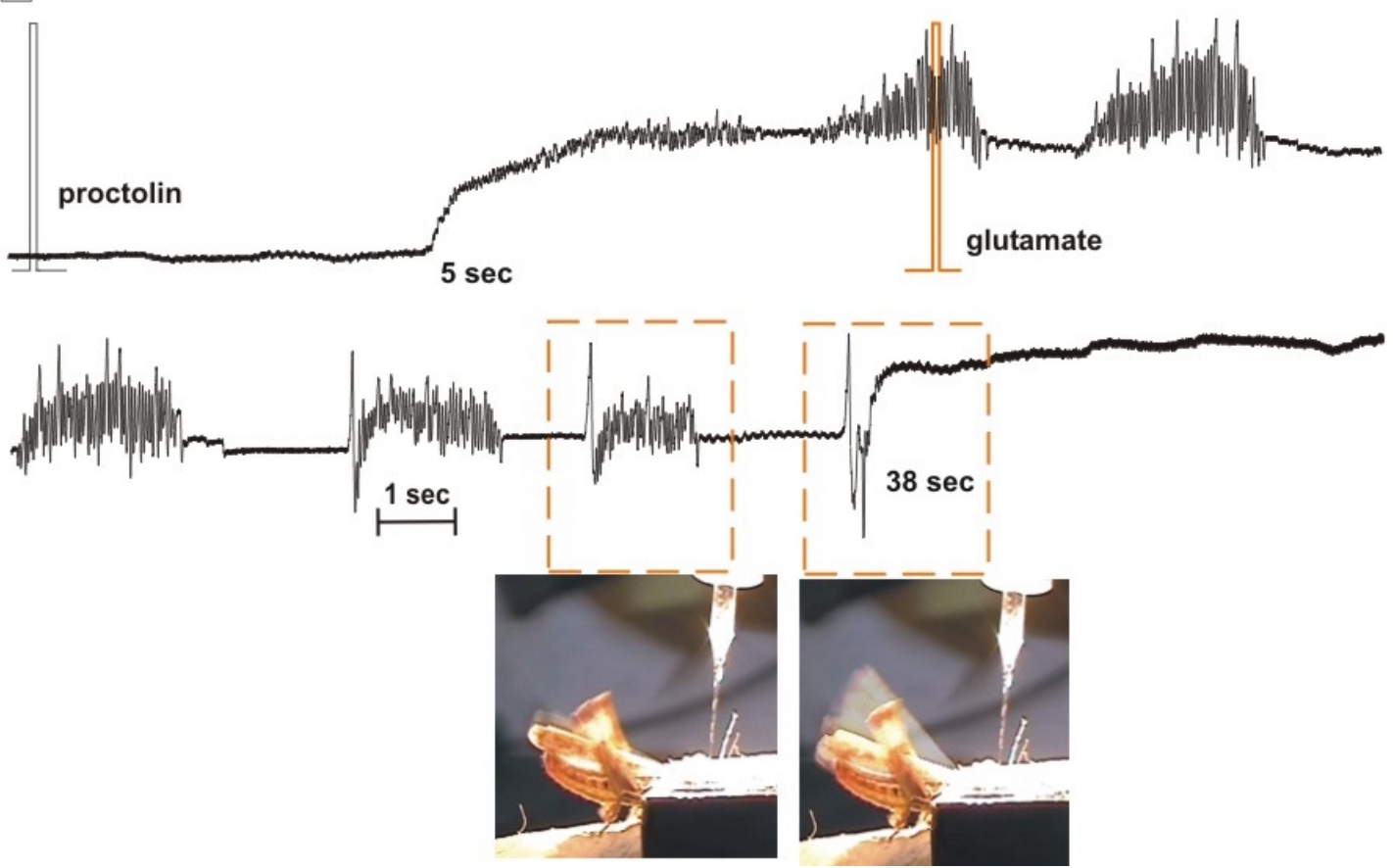

Fig. 3-72 HLs stridulation followed by wing stridulation, induced by injection glutamate during proctolin-stimulated singing (male, Ch.b.). A. Control pulse proctolin during the stridulation. B. Glutamate pulse (in red) during the stridulation induced wing-stridulating movements in 14 sec

In this experiment the performance of wing flapping after the leg stridulation was dependent on the interval between proctolin and glutamate injections. In other words, the time course of the second additional input might play a role in the switch mechanism between hindleg stridulation and the wing flapping. This result shows evidence of switch mechanism between two motor 
programs induced by proctolin and glutamate dependent on the time course of their release.

In the same preparation testing different interstimulus interval two proctolin pulses (as a control) at $20 \mathrm{sec}$ interval in between induced once wing flapping after 2 song sequences (Fig. 3-73). Interestingly, after the initial large-amplitude movement, stridulatory movements of very small amplitude during the wing flapping were performed by the left hindleg following by regular (Fig. 3-73).

In all experiments the wing flapping occurred after initial large-amplitude hindleg movements indicating a close relationship of both motor programs.

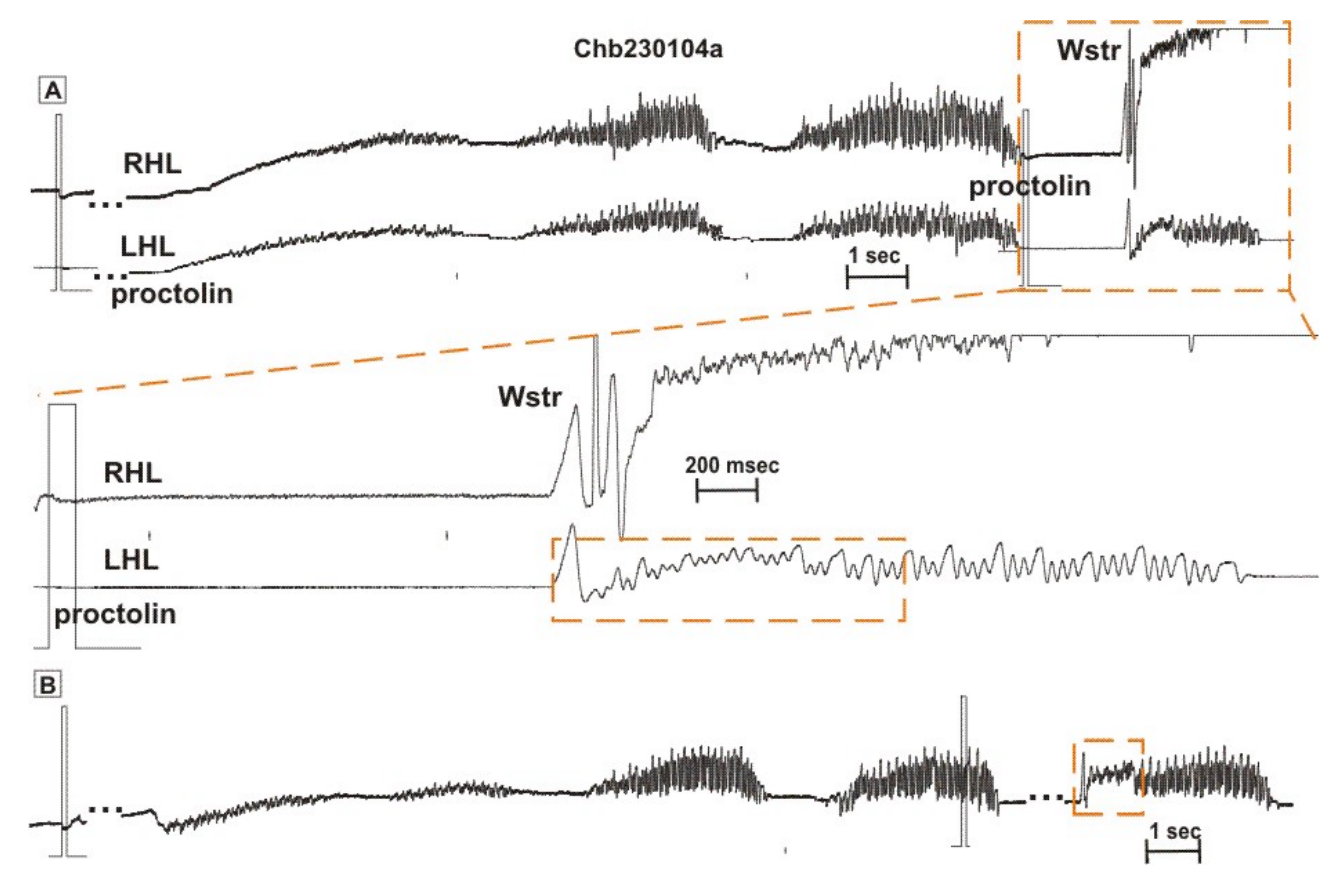

Fig. 3-73 A. Somng sequences followed by wing flapping produced by two proctolin injection (20 sec interval in between) (male, Ch.b.). In this stimulation regular stridulatory movements during the wing flapping were induced. B. In the next 7 stimulations was performed only leg stridulation but the similar initial stridulatory hindleg movements (red quadrtat) were performed as those during the wing flapping

In Fig. 3-74 shows wing flapping preceding by leg stridulation, stimulated pharmacologically, in two other males (Ch.b.).
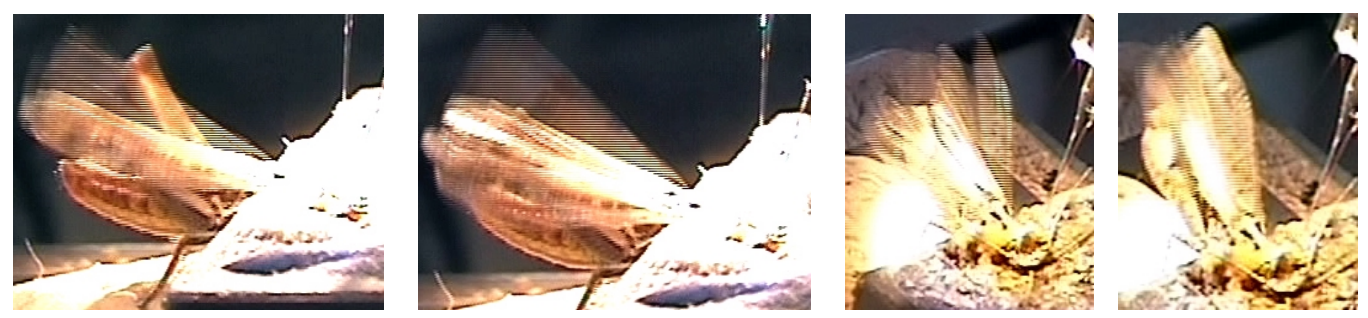

Fig. 3-74 Wing flapping after stridulation induced pharmacologically in males (Ch.b.) 


\subsubsection{Histamine}

To examine the involvement of histamine-gated $\mathrm{Cl}^{-}$channels in the control of stridulation, histamine was either co-applied to proctolin, using stimulation protocol 3-3-3, or injected, during proctolin-stimulated song sequences.

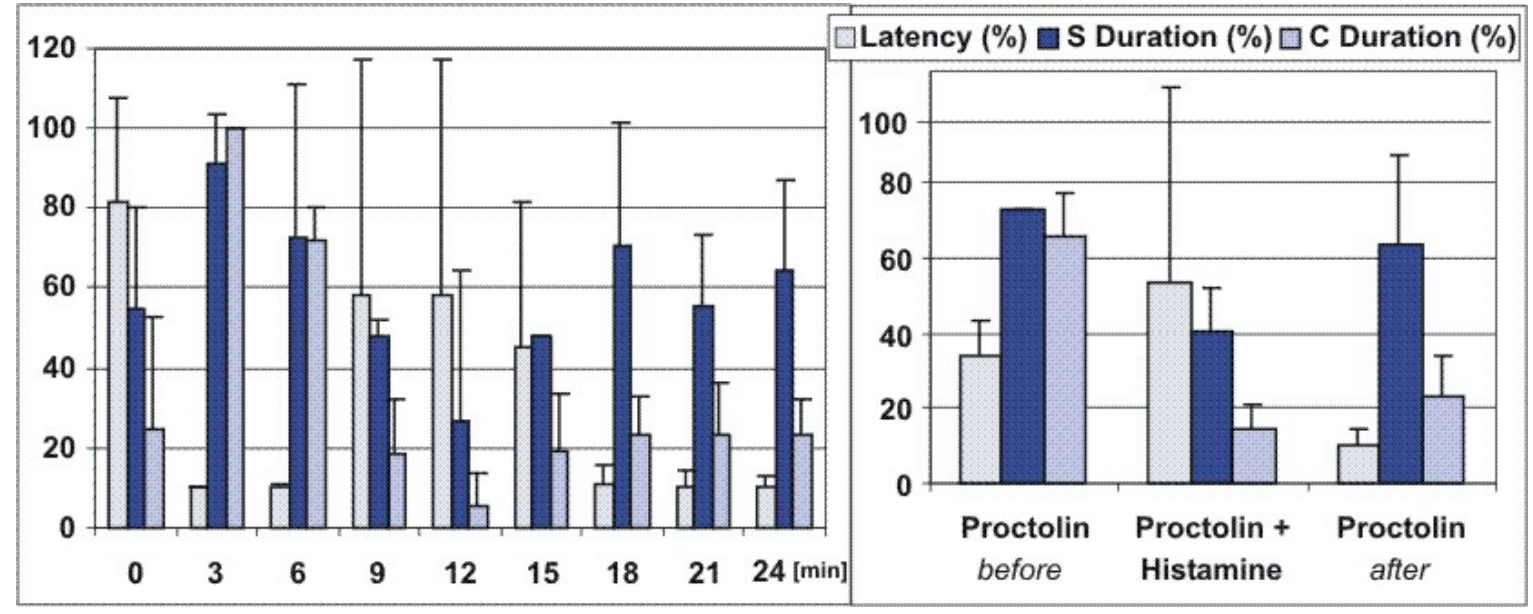

Fig. 3-75 Alteration of proctolin-stimulated stridulation by co-application of histamine (males, Ch.b., n=3) A. Protocol 3-3-3: 0,3,6, and 18,21,24 min- responses to proctolin; 9,12,15 $\mathrm{min}$ - responses to proctolin and histamine. B. Increase of the latency and decrease of durations during and after histamine co-injections. The $\mathrm{C}$ duration was in higher degree affected.

Proctolin-stimulated songs were altered by co-application of histamine (Fig. 3-75, A). The latency increased from 33,94\% ( $S D=9,23 \%$ ) before up to $53,91 \%(S D=51,44 \%, n=3, P>0,05)$ with histamine and subsequently decreased to $10,36 \%(S D=4,05 \%)$ after histamine co-injections. The S Duration changed respectively from $72,87 \%$ (SD=0,1\%), down to $40,80 \%$ (SD=11,06\%) and back to $63,68 \% \quad(S D=23,57 \%)$. The $C$ Duration was altered from $65,52 \%$ $(S D=12,21 \%)$, to $14,5 \%(S D=6,7 \%)$ with histamine and did not fully recover during the experiment but remained at $23,35 \%$ ( $S D=10,42 \%$ ) (Fig. 3-75, B). The differential changes in $S$ Duration and $C$ Duration was also a property of glycine-induced effects (Fig. 3-75, B) and could serve as an indication of changes of the internal time courses of the song sequences - the duration of the particular sequences and/or the pauses in between. One typical experiment is presented in the next figure (Fig. 3-76, A, B, C).

In response to the first proctolin injection the grasshopper performed long stridulatory activity $(\sim 0 \mathrm{sec})$ starting after a latency of $75 \mathrm{sec}$ (Fig. 3-76, A1). The second pulse $3 \mathrm{~min}$ later elicited faster response after $12 \mathrm{sec}$ and weaker late response (after $70 \mathrm{sec}$ ) (Fig. 3-76, $\mathrm{A}_{2}$ ). The $3^{\text {rd }}$ proctolin injection stimulated 


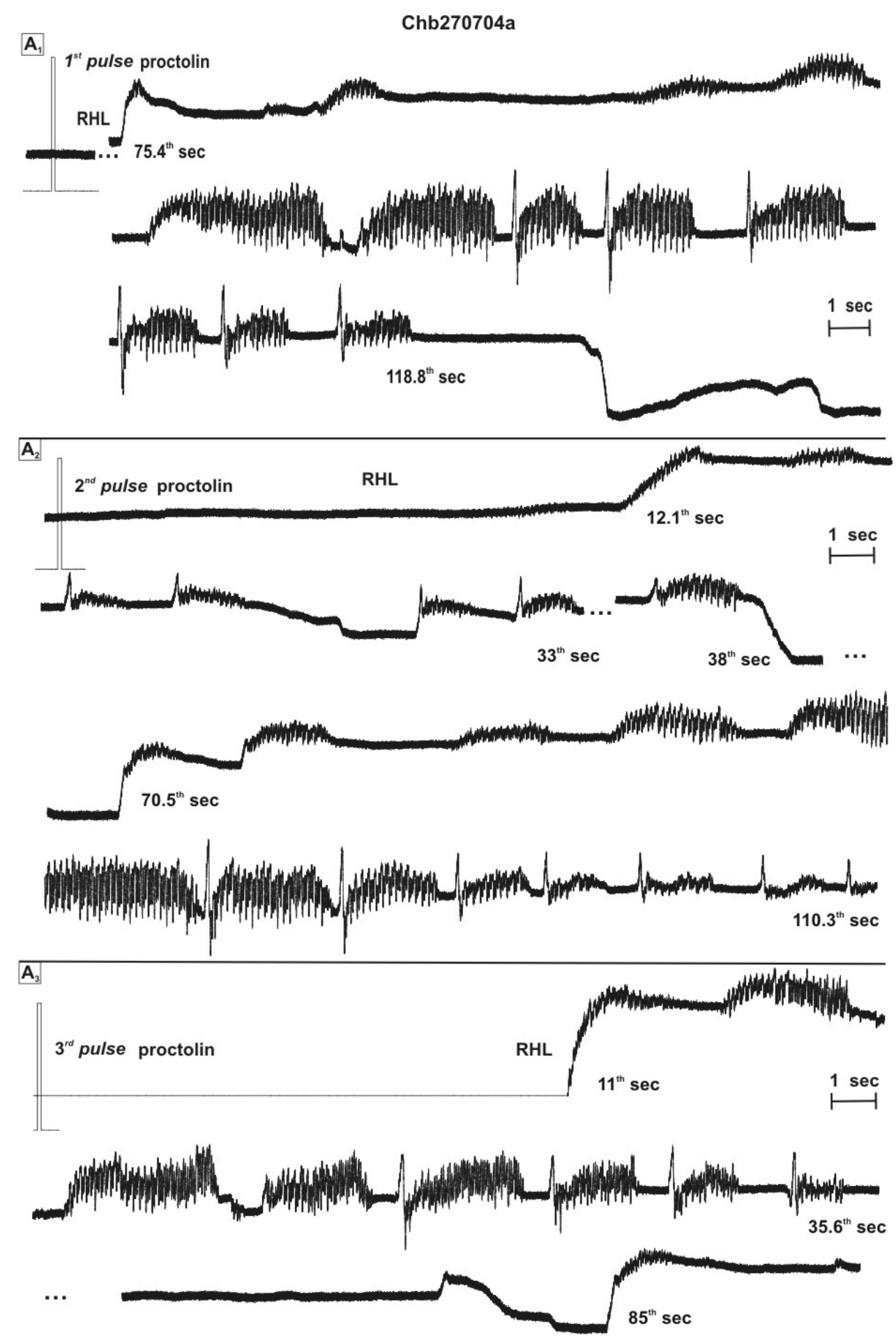

Fig. 3-76 A. Proctolin-induced responses in the initial phase of stimulation before the application of histamine (male, Ch.b.) (see the text)

only the earlier response, which became stronger and the later sequences disappeared (after $70 \mathrm{sec}$ ) (Fig. 3-76, $\mathrm{A}_{3}$ ). Interestingly, the silent period in between the earlier and the later song sequences was in the range of already demonstrated in previous experiments with glutamate (Fig. 3-68) and desensitization experiments (Fig. 3-17) 30-60 sec. 
In response to the following co-injections of proctolin with histamine early non-specific movements and late song sequences (120 sec) were performed

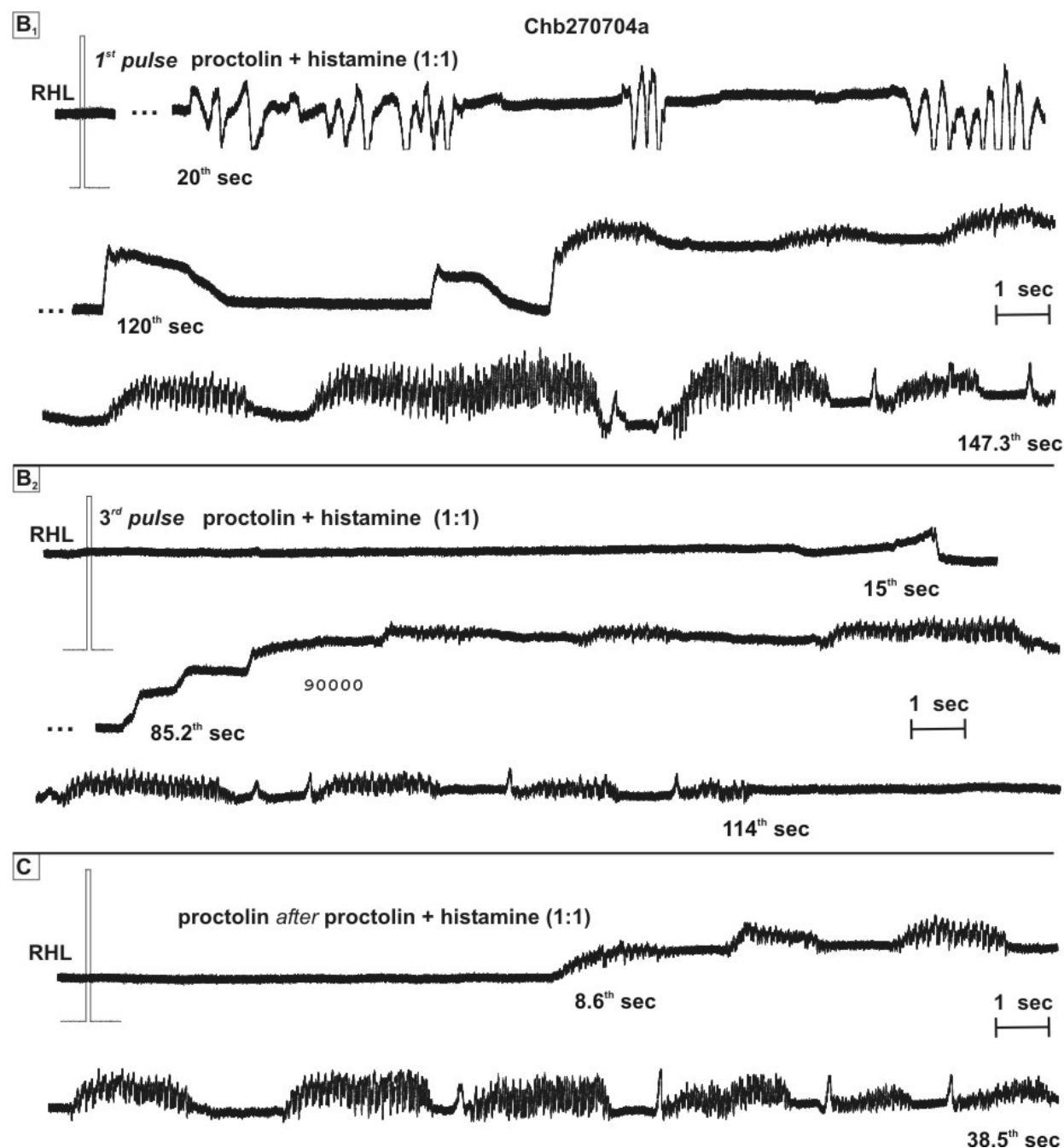

Fig. 3-76 B, C. The responses to proctolin injections before and after histamine co-injections were early and strong (A, C). In response to histamine co-injection late song sequences (85 sec) were performed and the amplitude of the stridulatory movements was two times smaller (B)

(Fig. 3-76, $B_{1}$ and $B_{2}$ ). The amplitude of the stridulatory movements decreased gradually to the $3^{\text {rd }}$ proctolin/histamine co-application (Fig. 3-76, $D_{1}, D_{2}$ ), while the duration of the stridulation remained very similar. The following proctolin pulses after proctolin/histamine COinjections stimulated only the early (8 sec) song sequences (Fig. 3-76, C).

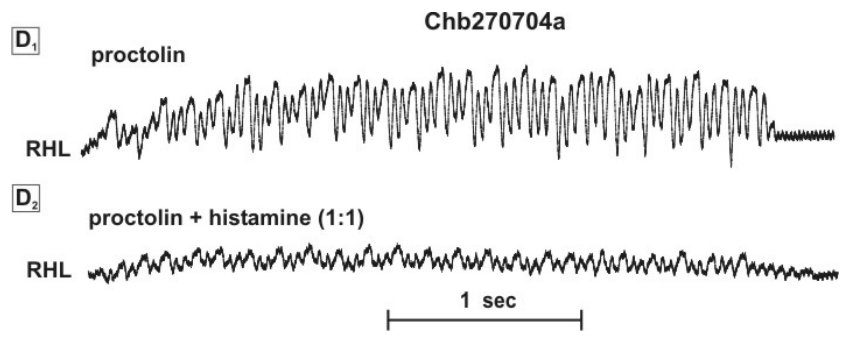

Fig. 3-76 D. The amplitude of the stridulatory HLss movements differed in response to proctolin and proctolin and histamine, respectively. 
The excitation was clear shifted from late responses to earlier ones throughout a silent phase $\sim 30-60 \mathrm{sec}$. Similar silent period between singing activity was shown in the previous section, examining the role of glutamate.

Another effect of histamine in some experiments was shortening of the duration of the song sequences, as it is shown in Fig. 3-77 A, B.

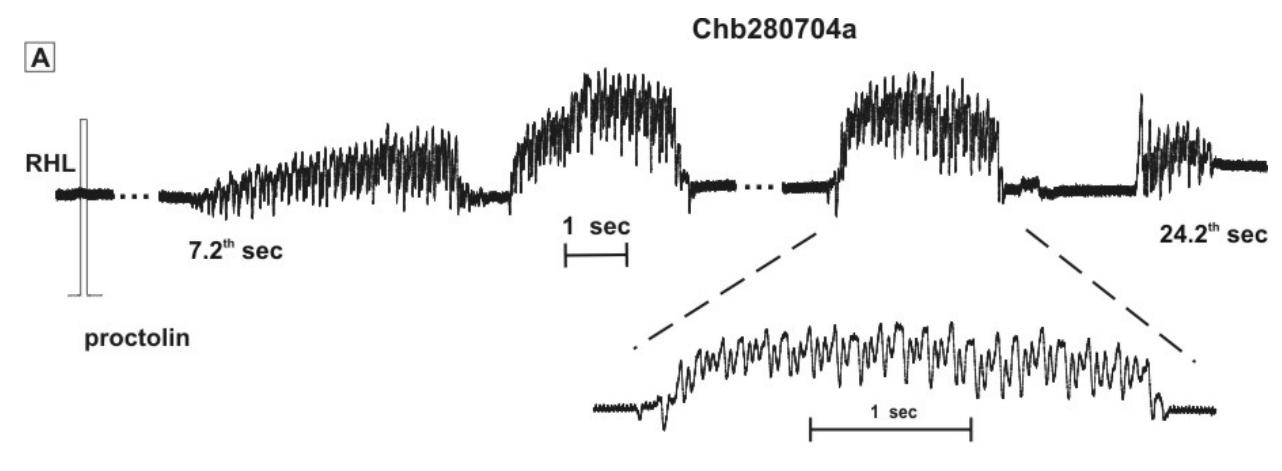

B

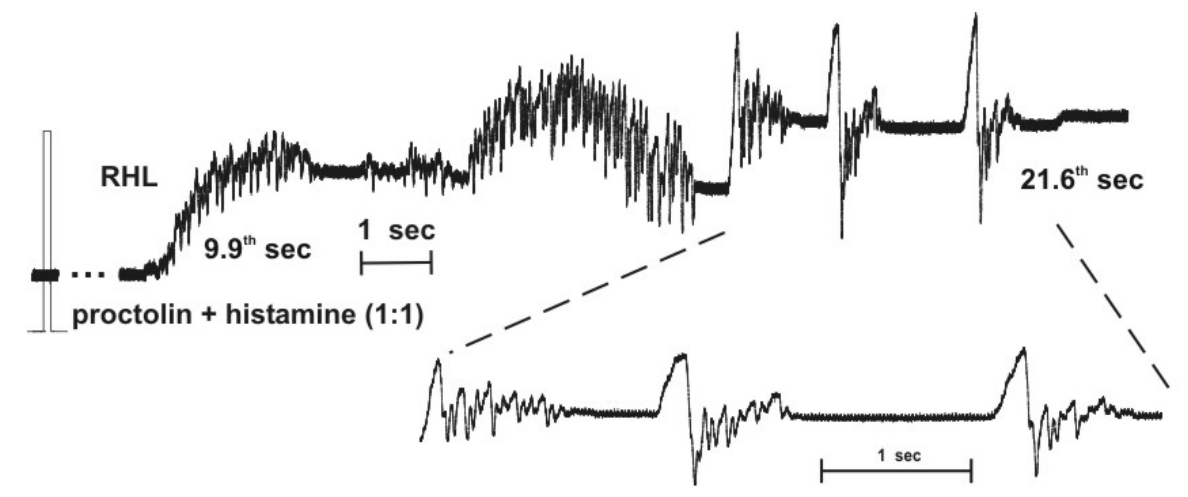

Fig. 3-77 Shortening of the song sequences stimulated by proctolin through co-application proctolin with histamine (male, Ch.b.). A. Song sequences stimulated by proctolin; B. Song sequences stimulated by co-injection of proctolin with histamine

When histamine was co-injected during proctolin-stimulated stridulation, 15 $\mathrm{sec}$ after the proctolin pulse, the duration of stridulation decreased. Fig. 3-78 A, $B$ presents the results from three experiment of this kind. Every 3 min two injections were applied at a $15 \mathrm{sec}$ interval, the first being always proctolin and the second being either proctolin (control) or proctolin/histamine mixture (test) (Fig. 3-78, A).

Histamine co-injection significantly decreased the duration (S Duration) of proctolin-induced singing from $97,87 \%(S D=2,72)$ before, down to $67,55 \%$ $(S D=0,12 \%, P>0,05, n=3)$ with and to $81,43 \%(S D=8,3 \%)$ after the coapplication. The values for C Duration were respectively $84,08 \%$ (SD=12,33); $45,63 \%(S D=14,47 \%)$ and $54,79 \%(S D=23,33 \%)$. The latency of the proctolin- 


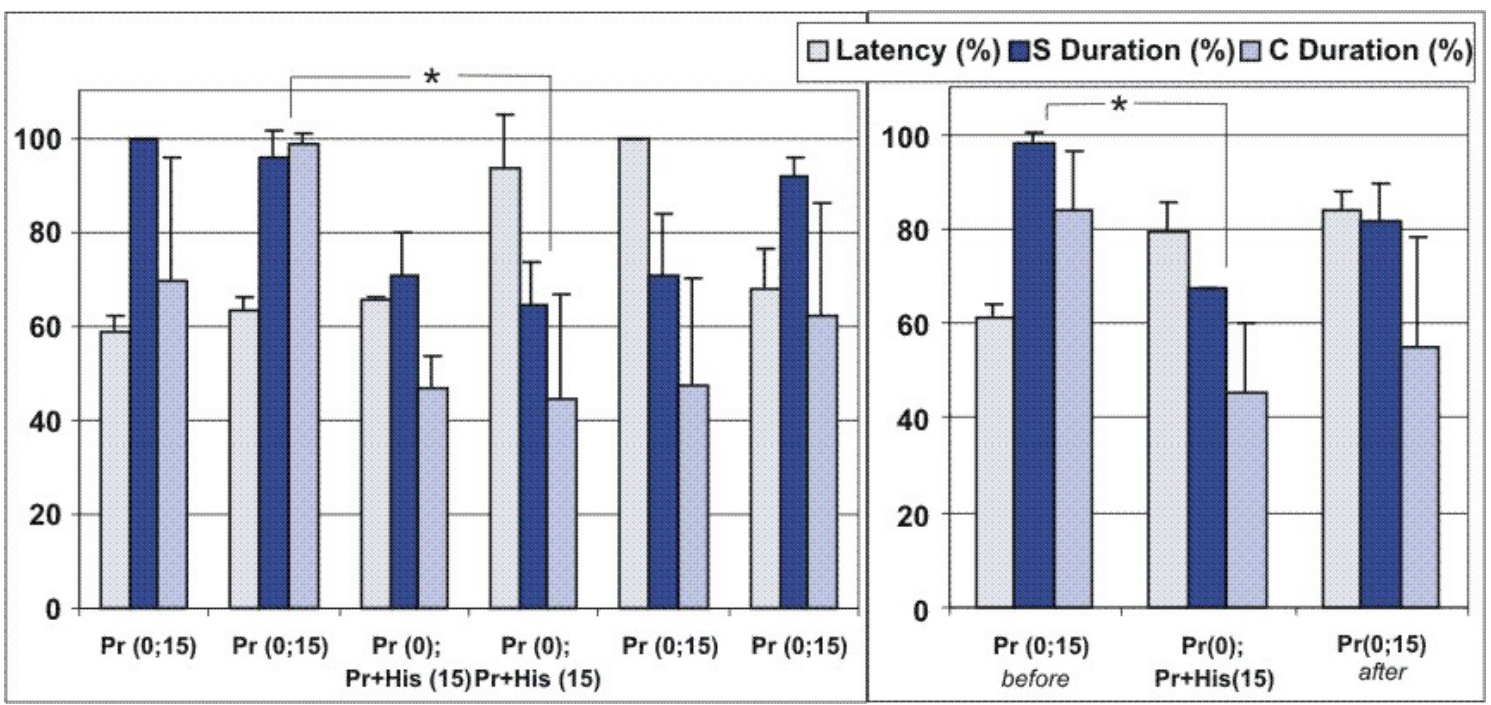

Fig. 3-78 Significant decrease of the durations of proctolin-triggered songs induced by co-injection of histamine (males, Ch.b., $n=3$ ). A. Protocol 2-2-2; B. Significant decrease $(P>0,05)$ of the durations

induced singing during the experiment increased from $61 \%(\mathrm{SD}=2,91 \%)$ up to the $83,97 \%(S D=4,14 \%)$ after the first co-injection of histamine (Fig. 3-78, A).

The effect of histamine was due to the termination of stridulation, approximately 1,5-2 sec after application of the proctolin plus histamine mixture was injected (Fig. 3-79, $A_{2}$ and $B_{2}$ ). This was similar in two different preparations.

The prematurely termination of the sequences occurred with latency of more than $1 \mathrm{sec}$ (Fig. 3-79), which is

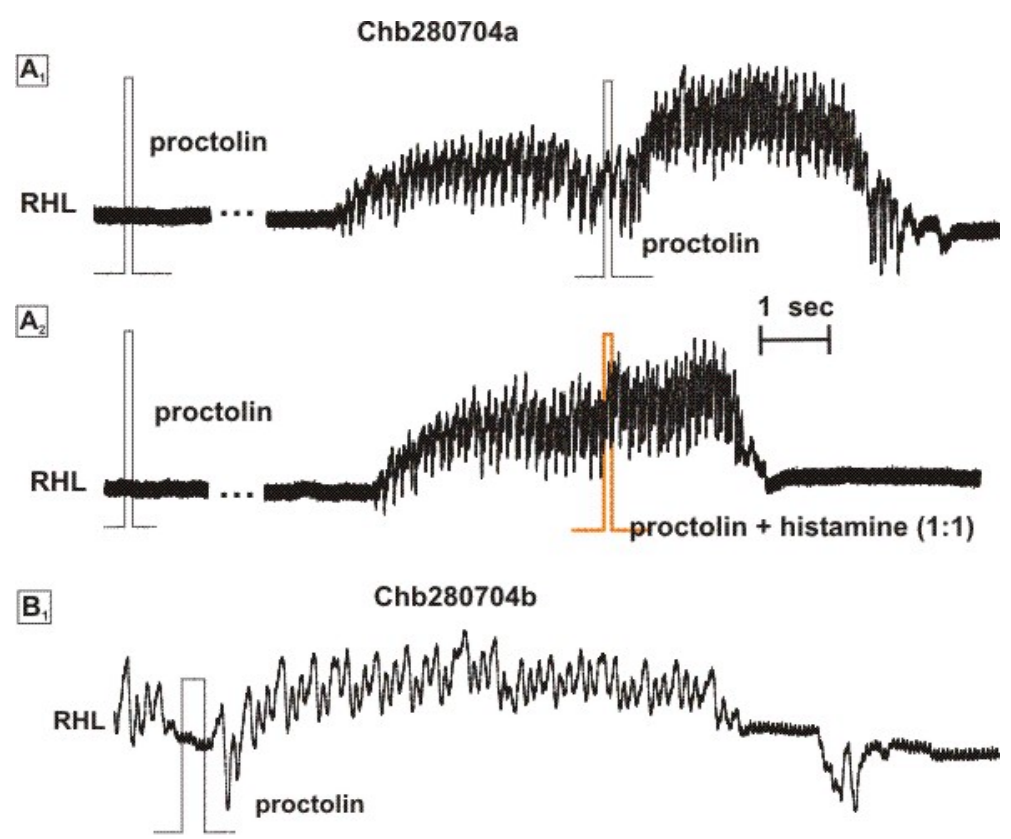

$B_{2}$

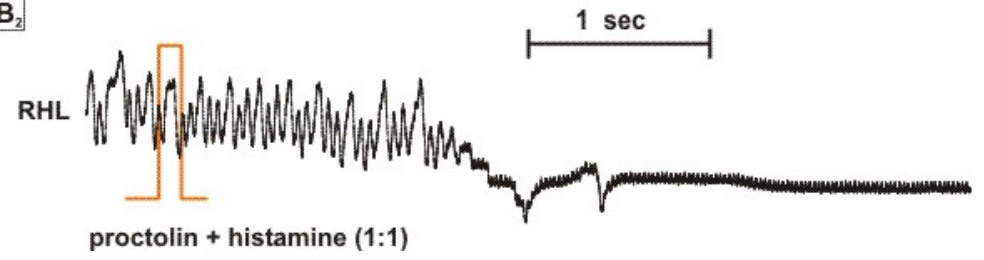

Fig. 3-79 Injection of proctolin and histamine during proctolininduced stridulation in two different males (Ch.b.). A1 and B1 control proctolin pulses, during the stridulation; $\mathrm{A} 2$ and $\mathrm{B} 2$ co-injection of proctolin and histamin (in red). The latency of histamine effect was longer than $1 \mathrm{sec}$. 
unlikely to be mediated by fast hyperpolarizing $\mathrm{Cl}^{-}$channels, locating on the same neurons that drive stridulation upon proctolin stimulation. The time course of histamine effect may suggest that either metabotropic receptors (mHisRs).

In other experiment histamine co-injections during the proctolin-stimulated singing induced shorter song sequences (Fig. 3-80, A, B).

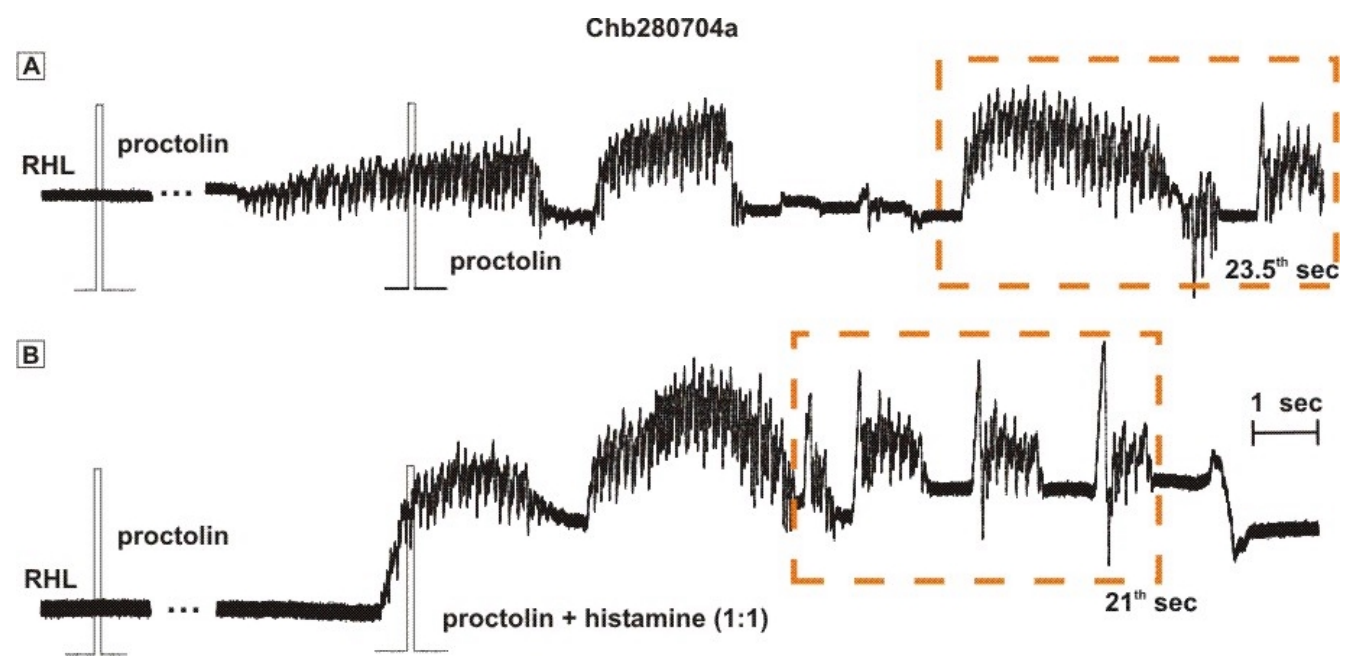

Fig. 3-80 Histamine co-injected with proctolin during the proctolin-stimulated stridulation decreased the durations of the song sequences of $1^{\text {st }}$ order (male, Ch.b.) A. Song sequences stimulated by two pulses proctolin with $10 \mathrm{sec}$ interval in between (control); B. Shorter song sequences of $1^{\text {st }}$ order induced by co-injection of histamine with proctolin during ongoing singing

The main problem using natural transmitter within the stimulations is that the mode of action can be triggered by both excitatory and inhibitory, fast and slow mechanisms, which might be strict coupled, producing complex responses. All substances tested in these series experiments glycine, glutamate, histamine and GABA activate not only $\mathrm{Cl}^{-}$channels but gated also metabotropic receptors, coupled to different second messengers cascades. Glycine and glutamate can be ligands of ionotropic cation channels, triggering fast depolarization.

In order to examine the general role of only $\mathrm{Cl}^{-}$channel activation in the control circuits for singing behavior an activator of these channels, the insecticide ivermectine, was tested. 


\subsection{6 $\mathrm{Cl}^{-}$channels activator ivermectine (IVM)}

Stridulation in male Ch.b. has previously been initiated through picrotoxin (PTX), a well-known $\mathrm{Cl}^{-}$channel blocker. Initiating stridulation by blocking $\mathrm{Cl}-$ channel-mediated inhibition suggested, that stridulation is tonically suppressed (Heinrich et al., 1998).

The patterns of PTX-stimulated songs have been less specific and regular when compared with those of muscarine-stimulated activity.

In this study the main ligands (glycine, glutamate, histamine, GABA) of the known $\mathrm{Cl}^{-}$channels in insects showed more complex mode of action, altering few songs parameters, including inhibition. In order to examine the net effect of $\mathrm{Cl}^{-}$channels activation, thus the role of the fast inhibition, on the proctolinstimulated stridulation, the insecticide ivermectine (IVM), one activator of $\mathrm{Cl}$ channels, was co-injected with proctolin. If the $\mathrm{Cl}^{-}$channels are involved in the termination of the song sequences or decreasing the basal level of excitation, induced by proctolin or muscarine, their activation should suppress both proctolin and muscarine responses.

Ivermectine (IVM), $\mathrm{Cl}^{-}$channel activator, had no effect on proctolinstimulated stridulation in Ch.b. No quantitative changes in the parameters latency and duration were observed (Fig. 3-81, A, B) and the patterns of stridulatory hindleg movements were not altered.

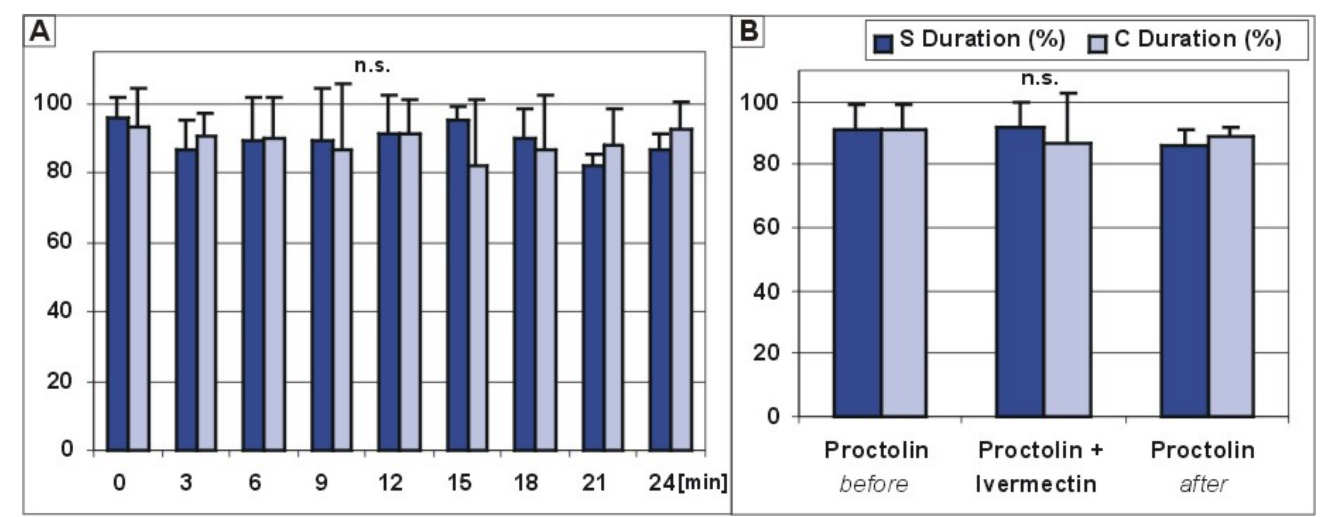

Fig. 3-81 No effect of the insecticide ivermectine $(0,1 \mathrm{mM}), \mathrm{Cl}^{-}$channels activator, on proctolin-stimulated stridulation (males, Ch.b., n=3) A. Protocol 3-3-3: 0,3,6 and 18,21,24 min - responses to proctolin; 9,12,15 $\mathrm{min}$ - responses to proctolin/ivermectine mixture. B. Averaged durations before, with and after IVM co-injections. 


\subsubsection{Other neuroactive substances}

Co-injections of other transmitters and neuroactive substances with proctolin were tested but not systematically carried out. Octopamine, coapplied to proctolin strengthened the stridulatory movements and affected only the latency of the response (males, Ch.b., $\mathrm{n}=2$ ). Serotonin, likewise, lengthened the response latency two and a half times (males, Ch.b., $\mathrm{n}=2$ ). $\mathrm{PACOCF}_{3}$, an inhibitor of $\mathrm{PLA}_{2}$ (arachidonic acid second messenger pathway) decreased slightly and reversibly the duration of proctolin-induced stridulation from $74 \%$ to $52 \%$, using protocol $3-1-4$, in males Ch.b. $(n=3)$.

Preliminary experiments were carried out to examine, whether the pharmacologically stimulated female stridulation is suitable for an examination of the second messenger systems coupled to proctolin receptors.

$\mathrm{PACOCF}_{3}$, blocker of $\mathrm{PLA}_{2}$ decreased the duration, but not the latency of proctolin-stimulated singing from $80 \%(\mathrm{SD}=2 \%)$ down to $51 \%(\mathrm{SD}=25 \%)$ in females Ch.b. $(\mathrm{n}=2)$. The effect reversed to the next proctolin injections $(77 \%$, $\mathrm{SD}=33 \%$ ), using protocol 3-1-4 (Fig. 3-82).

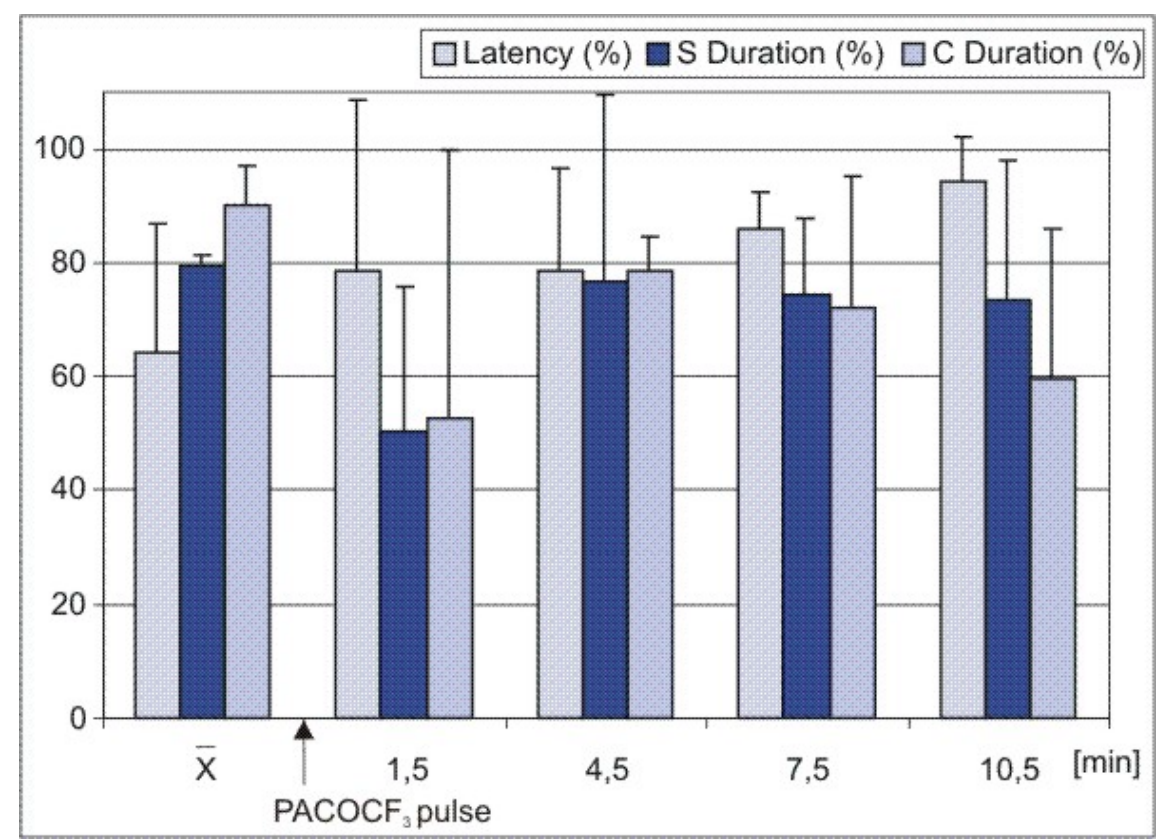

Fig. 3-82 $\mathrm{PLA}_{2}$ inhibitor $\mathrm{PACOCF}_{3}$, decreased the durations of proctolin-stimulated stridulation in females Ch.b. $(n=2)$ 


\section{Discussion}

\subsection{Proctolin-elicited singing behavior in Ch.b. - comparison to the} natural one

Proctolin injected alone into the protocerebrum initiates and maintains singing in males of Ch.b. similar to the natural stridulation of this species. The stridulatory activity lasts $5-30 \mathrm{sec}$, depending on the conditions of stimulation (site of injection, volume of injected proctolin, time after previous stimulation etc.) and the behavioral status of the animal. Two level of excitation were observed in some cases even uncoupled (Fig. 3-13) that were named initiation and maintenance stage of stridulation. The initial one or two song sequences are longer than the following ones and are accompanied by a rise of the hindlegs' position. The initiation stage turns into the maintaining stage without any additional stimuli. The hindlegs (HLs) are now kept in elevated (up) position and perform shorter sequences (1-3 sec). The duration of the sequences (3.2), induced through proctolin are $\sim 1-2 \mathrm{sec}$ shorter than the natural sequences (3.1). The chirp structure of proctolin-induced songs does not differ from the natural one. Most observed chirp pattern consist 6 syllables per chirp -2 small up- and down- movements and one with larger amplitude that gives the pause. The inter-chirp pauses and the constant pause/chirp ratio ( 1:5) performed from a single hindleg (patern I), no matter of the level of excitation is one of the main features of the male Ch.b. song (Fig 3-2) which make it high specific and unique among the other singing gomphocerines. The highly specific complex rhythmicity of the stridulatory movements has been proposed to be the intrinsic property of the thoracic CPG activity. The phase shift between the left and right movements and the long lasting up-stroke movements, performed by one of the legs (pattern II) fills the pauses with sound. The females prefer pattern II then pattern I if they are stimulated acoustically (von Helverson, 1997). Nevertheless pattern II does not change the constant pause/chirp ratio performed by each hindleg. Proctolin elicits both singing patterns I and II each being produced by one of the HLs. The HLs usually alternate the singing patterns every few sequences of $1^{\text {st }}$ order in response to proctolin, similar to the stridulation of freely behaving animals (3.1). 


\subsection{Song types and other courtship elements stimulated by proctolin}

Proctolin can induce calling song as well as courtship song (3.2). In section 3.2 the question was raised whether at all a specific rivalry song has ever been stimulated in Ch.b. Usually the freely behaving males respond to both female and male singing. The competition between males is high but overt antagonistic behavior is rare and may only occur in cases of very close contact between two males.

The main difference between the song types that had been described in several works (Reis, 1995; Ocker, 1995) is shorter sequences for both courtship and rivalry songs, compared to calling songs. Following pharmacological stimulation, only in few grasshoppers performed rivalry-like song sequences with the typical vigorous beginning and subsequent gradual decrease of sound intensity. In all other preparations shorter sequences were stimulated with high amplitude first up- and down- movement (Fig. 3-9) but the sound intensity did not decrease during the sequences.

A similar pattern was observed by Wenzel (2000) and interpreted as rivalry-like song sequences. However, such patterns seemed to appear on a regular basis after strong stimulation with muscarine, indicating that they may not reflect a switch to rivalry song patterns but instead represent courtship of high intensity. Such a "development" of patterns during prolonged courtship stridulation has been described for various species (Heinrich et al., 2001). Rival songs, distinguished from other song types by short sequences, high initial intensity and decreasing intensity during the sequence may have only been elicited in two preparations. Since it has not been able to elicit genuine rival songs by pharmacological brain stimulation in other species (Heinrich, personal communication), this song type may require specific conditions for stimulation and is therefore difficult to release. Own observations suggested that courtship sequences might be of shorter duration than the sequences of calling song (1-2 sec courtship song vs. 2-4 sec calling song). A comparison of proctolin- and muscarine-stimulated song also revealed a difference in the duration of individual sequences (1-3 sec with proctolin vs. 1-10 sec with muscarine). One may speculate that co-transmission of proctolin in addition to cholinergic excitation could initiate the switch between calling and courtship behavior, when a female show copulatory readiness (Fig. 3-4) This idea is supported by the 
appearance of additional behavioral elements of courtship, such as wing flapping, precopulatory movements and other (section 3.6.4) only after costimulation of muscarine with proctolin or proctolin and other transmitters, while these additional patterns remained absent when muscarine was exclusively applied.

Furthermore, the courship song should be observed only together with other behavioral actions occurred during the phonotactic mate approach after the turning female response (Fig. 3-3). Courtship elements, such as laterization, moving forward (phonotaxis), jumping, wings movements in step-wise manner owe to the perception and recognition of the con-specific female song (Lautschema) from the brain acoustic centers and orientation mechanisms. In other words, recognition and integration of specific sensory information (auditory and visual) results in the complex subsequently performed phonotactical approach and courtship song.

Brain neurons that co-release proctolin to other transmitters (ACh, glutamate) within the control circuits for stridulation may therefore be activated by sensory (auditory and visual) cues, which signal the responding female.

The sound production controlling from a population of command neurons might be subordinated to a brain center that consists proctolinergic neurons. mAChRs activation may lead to prolonged calling singing, depending on abiotic factors (temperature, light etc.). This stridulatory activity should be combined and coordinate with another complex behavioral programs (sex-specific song recognition, orientation and loco-motor activity) and switching between them in a step-wise manner, without decrease of the basal excitation to sing.

Proctolin triggers courtship song, which is followed by silent period (desensitization or refractory periods), which duration depends on the preceding stridulatory activity (section 3.6.4). During the silent periods perception, orientation and active phonotaxis could be activated.

Pharmacologically (co-injections) induced delay of proctolin-stimulated stridulation without affecting the song duration and intensity (without decrease of the basal excitation) (section 3.5.2.4) supports the assumption that proctolin may play a role in the control of step-wise alternations between the courtship song and the phonotactical approach. 
It has been previously demonstrated that a brain population of command neurons exist, each for a certain song type in O.v. (Hedwig and Heinrich, 1997). The fact that mAChRs activation is not necessary for proctolin-stimulated stridulation (section 3.4.5) supports the idea that distinct proctolinergic and cholinergic (mAChRs) neurons may induce different song types in Ch.b. This hypothesis should be further proved combining this pharmacological approach with electrophysiological studies.

\subsection{To dose-effects of proctolin mode of action}

Dose-dependent effects were examined in the light of the main presumption that the neuronal network controlling the sound production responds different to the same excitatory inputs in stridulating and silent grasshoppers. The increased arousal produced by the repeated stimulation in the beginning of each experiment supports this presumption. Accurately experiments may have carried out, than and than only stable responses to a given stimulation parameters have been achieved, named in section (section 3.2.3) maintaining stimulation phase. Experiments assessing the effects of different volumes, interstimulus intervals and different test substances were only performed after the initial stimulation phase, when stable responses occurred following each additional stimulation.

In particular protocol dose effects were examined only after receiving at least 3 stable responses to identical stimulations. Subsequently the responses to two stimulations with test parameters (e.g. number of the pulses) were compared to the initial responses. In the end of series of stimulations two responses to the control stimulation parameters were again recorded. If the latter differed significantly from those in the beginning of the experiments, the results were not included in the evaluations, because other factors, rather than the dose of proctolin, may have affected the responses.

Slight decrease ( $20 \%)$ of the proctolin-stimulated response following by lack of response with decreasing the dose injected (Fig. 3-15), may be caused if the responses stimulated are short (1-2 sequences) around the threshold of initiation. Than can be observed such "all or none" dose-dependent responses. Interestingly, proctolin-stimulated long responses (more than 5 sequences) 
showed the same dose-dependent behavior. This suggests that proctolin receptor population is restricted and triggers responses, which are almost doseindependent under our stimulation conditions.

\subsection{Temporal aspects of proctolin-stimulated stridulation}

For studies of potential excitatory or inhibitory effects of pharmacological agents known to interfere with intracellular signaling pathways, it was crucial to maintain a constant level of basal excitation resulting from previous injections of proctolin. Besides the methodological problems mentioned in section 3.3 it was obligatory to study the time course of proctolin stimulations to accumulate and maintain a consistent level of excitation resulting from appropriate intervals between individual stimulation pulses of an experimental series. The optimal interstimulus interval was found to be $3 \mathrm{~min}$ (3.2.5.1). Shorter interstimulus intervals (3.2.5.2) resulted in decreased durations of proctolin-stimulated stridulation, most likely caused by mechanisms of receptor desensitization, while longer intervals increased the duration of experimental series, which increased the probability of unspecific changes in the general condition of the preparation.

In freely behaving animals calling sequences $2^{\text {nd }}$ order ( $=$ complete normal song) consist of $3-5$ sequences $1^{\text {st }}$ order and last $\sim 15-20 \mathrm{sec}$. After that the males make a longer pause ( $30 \mathrm{sec})$ and walk, jump etc. before the next song is performed (personal observations).

Similar to this, the shortest interval between two proctolin stimulations, which successfully can initiate stridulation was found to be $\sim 30 \mathrm{sec}$. In contrast to O.v., where repetitive injections of ACh every $15 \mathrm{sec}$ can trigger singing, proctolin stimuli during the desensitized state cannot initiate any stridulatory movements. The latter indicates that PRs might be desensitized and cannot be stimulated for a period of time. Proctolin stimulation can initiate stridulation only if the interstimulus interval is longer than $30 \mathrm{sec}$ (3.2.4.2). Moreover increasing the interstimulus interval up to ( $\sim 2 \mathrm{~min})$, increases the duration of stridulation stimulated by the proctolin pulses. The latter suggests that the desensitization effects outlast the period of $30 \mathrm{sec}$. 


\subsection{Possible involvement of receptor desensitization in the inactivation of proctolin-induced singing behavior (Ch.b.)}

The desensitization of neuropeptide receptors is caused by receptor phosphorylation and/or by receptor down regulation (Garland et al., 1996; McConalogue et al., 1998; Nässel, 2002).

A possible involvement of receptor desensitization as being the reason for decreased potency of proctolin to stimulate singing with intervals shorter than $30 \mathrm{sec}$ is suggested (section 3.2.5.2). Blockade of both types AChRs (nicotinic and muscarinic), trough dTC and scopolamine, respectively did not affect the desensitization effects of repetitive proctolin stimulations (data not shown), indicating that indirect influence from cholinergic transmission on the desensitization phenomenon could be excluded.

Proctolin action can also be terminated (Wood and Nusbaum, 2002; Mazzocco et al., 2003) or tuned (Isaac, 1987; 1997) by the activity of a membrane peptidase. Peptidase activity can limit the spatial influence, intensity, and the duration of neuropeptide action (Agnati et al., 1995; Saleh et al., 1996; Zappulla et al., 1999).

A Drosophila melanogaster cDNA clone (GH01916) encoding a putative 723-residue long (82 kDa) protein (CG 7415) and displaying 50\% identity with mammalian cytosolic dipeptidyl aminopeptidase (DPP) III has been functionally expressed in Schneider S2 cells. Immunocytochemical studies using anti-(rat liver DPP III) Ig have indicated the expression of this putative DPP III at the outer cell membrane of the transfected cells. The partially purified enzyme has hydrolysed the neuropeptide proctolin (Arg-Tyr-Leu-Pro-Thr) at the Tyr-Leu bond $(\mathrm{Km} \sim 4 \mu \mathrm{M})$. In addition, low concentration of the specific DPP III inhibitor tynorphin has prevented proctolin degradation $\left(\mathrm{IC}_{50}=0.62 \pm\right.$ $0.15 \mu \mathrm{M})$. These results have constituted the first characterization of an evolutionarily conserved insect DPP III that is expressed as a cytosolic and a membrane peptidase involved in proctolin degradation (Mazzocco et al., 2003).

The phenomena of termination of the proctolin mode of action reveals another field of further pharmacological investigations, using our model system.

Pressure injection of tynorphin can be used to block the activity of DPP III in order to examine its role in the termination of proctolin action. 
4.6 The role of cholinergig transmission on the time course of proctolin-stimulated stridulation

In O.v. nAChRs-activation (ACh and nicotine) leads to a short singing after a very short latency $(\sim 0,5 \mathrm{sec})$, while the mAChRs-activation (muscarine and pilocarpine) induces prolonged singing (3-60 min) (Heinrich et al., 1997). Repetitive ACh stimulations, every $15 \mathrm{sec}$, cause arousal of the duration of stridulation. The latter additional excitation has been blocked by scopolamine, inhibitor of mAChRs (Heinrich et al., 1997), suggesting that the nAChRsactivation may initiate, while mAChRs-activation maintains and potentiates the stridulation. It remains to be demonstrated, whether the blockade of $\mathrm{nAChRs}$ can affect muscarine-stimulated stridulation in O.v. In addition, nothing is known about the role of AChE modulation in the initiation and maintenance of stridulation in this species.

In contrast to O.v., in Ch.b. ACh failed to trigger stridulation under any stimulation conditions tested, including repetitive injections every $15 \mathrm{sec}$, demonstrated by Furthermore, ACh applications to the same sites in the brain, where proctolin triggered singing also failed to initiate any song sequences. Coinjected with proctolin ACh had a suppressing effect, leading to decrease of the duration of stridulation (section 3.4.6). By examining the influence of AChRsactivation on proctolin-stimulated stridulation, only the blockade of nAChRs and not of mAChRs affected proctolin-stimulated singing. Under different stimulation conditions the blockade of nAChRs caused either inhibition or potentiation of the proctolin-induced stridulation (section 3.4.6.1). The blockade of nAChRs also potentiated muscarine-stimulated stridulation, suggesting a different role for nAChRs-activation in the cephalic control of of stridulation in Ch.b., than originally described role in O.v. It is proposed that $\mathrm{nAChRs-activation} \mathrm{may} \mathrm{have}$ a role in the modulation and not in the initiation of the stridulation in Ch.b.

In addition, modulation of AChE activity clearly affected - long potentiation, preceding by inhibition - both muscarinic and proctolinic responses. Nevertheless this pharmacological approach cannot offer information about the localization of mAChRs, the fact that AChE blockade inhibited the muscarinic responses (section 3.4.6.2), possible presynaptically localization of mAChRs within the neuronal circuit, controlling the stridulation in Ch.b., cannot be excluded. 
Brought together these data suggests that fine-tune modulation of $\mathrm{AChE}$ activity may influence the initiation and maintenance of the stridulation.

It has been postulated that nAChRs-mediating excitation may trigger single sequences in Ch.b., because similar mechanisms have been described in O.v. (Heinrich et al., 1997; Wenzel et al., 2000, 2002). The received results do not support this proposal. The data support previous report, that exclusively activation of nAChRs in Ch.b. and other species with more complex rhythms of stridulatory hindleg movements than it is in O.v., cannot elicit stridulation (Heinrich et al., 2001). These species may require a stronger contribution of basal excitation, mediated by muscarinic, proctolin, glutamate and probably additional metabotropic receptor systems.

In the experiments where AChE activity was blocked through co-injection of eserine with proctolin no silent pauses were observed and a prolonged artificial singing was induced (section 3.4.6.2). The blockade of AChRs includes a prolonged ACh signal on AChRs. This might cause a specific desensitization of nAChRs and indirect effects on the time courses of proctolin-stimulated singing.

To clarify principal mechanisms underlying receptor desensitization, mechanisms of nAChRs-desensitization and its modulation are presented in more details.

\section{Desensitization and modulation of nAChRs}

nAChRs progressively desensitize by prolonged activation through ACh. Recordings of a nicotinic receptor channel with the patch clamp technique (whole cell configuration) show a progressive diminution of the total current $I_{\text {ACh }}$ in the presence of a high and constant concentration of ACh. (Fig. 4-1, from Clapham et. al., 1984).

The process of desensitization appears slowly and is slowly reversible. This is an intrinsic property of the receptor protein. The rate of desensitization of the nicotinic receptor seems to be related to its state of phophorylation. In fact, studies of the ionic flux through

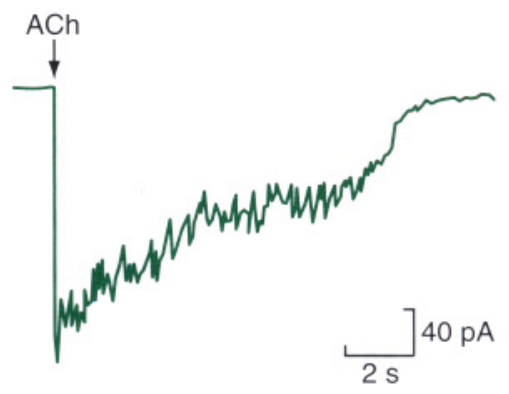

Fig. 4-1 Desensitization of $\mathrm{nAChR}$ by prolonged activation of ACh (patchclamp whole cell configuration). (from Clapham et al., 1984). nicotinic receptors incorporated into liposomes have shown that an increase in the level of phosphorylation of the receptors by cAMP can augment the 
desensitization rate of these receptors. In the neuromuscular junctions a peptide present in the motoneurons is co-released with ACh. This peptide, CGRP (calcitonin gene-related peptide), is capable of increasing the level of CAMP in cultured embryonic muscle cells, consequently increasing the number of phosphorylated nicotinic receptors (Fig. 4-2)(Hammond, 2001).

However, nothing is known, whether similar modulation of nAChRs-desensitization through neuropeptides may exist in CNS of vertebrates and in insects.

To summarize the $\mathrm{nAChR}$ properties leading to their desensitization:

\[ \text { nAChRs can exist } \]
spontaneously in the four freely
interconvertible and discrete

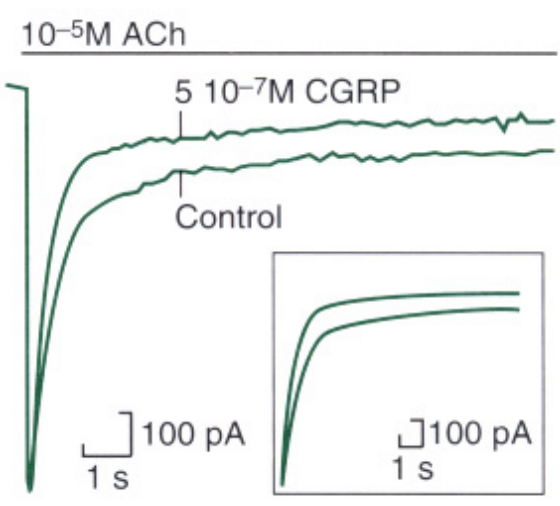

Fig. 4-2 Modulation of nAChR desensitization by

conformational states, even in the absence of ligand:

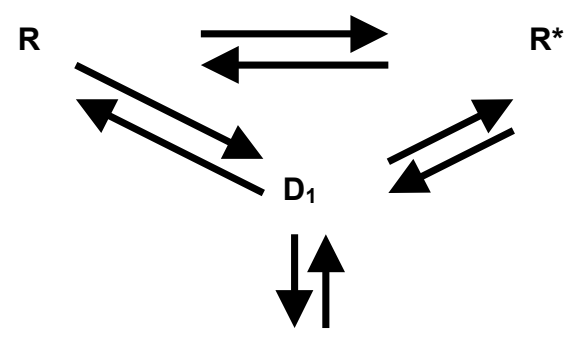

$\mathrm{D}_{2}$

$\left(R^{*}, A R^{*}, A_{2} R^{*}\right.$ - open states; $R, A R, A_{2} R$ - closed states; $D_{1}, A_{1}, A_{2} D_{1}$ and $D_{2}, A_{2}, A_{2} D_{2}-$ desensitized states; $\mathbf{A}-\mathbf{A C h}$ or any nicotinic agonist)

- The affinity and activity of the stereospecific sites carried by the nAChR differ between these four states

- Gating of the nAChR cannot be viewed solely as a ligand-triggered process but as reflecting an intrinsic structural transition of the receptor molecule, which may even occur in the absence of ligand. Moreover, at low concentrations, desensitized states can be stabilized under conditions of negligible channel opening.

- Upon two molecules ACh binding, each nAChR undergos fast activation leading to an open-channel state (in msec range), and slow desensitization reaction leading to a close-channel state reflectory to activation. Fast desensitization occurs in the $0,1 \mathrm{sec}$ range and slow desensitization in the minute range (Hammond, 2001). 


\subsection{Necessity of PRs for muscarine-stimulated stridulation}

Unfortunately, specific blocks of PRs and their impact on muscarinestimulated stridulation were not tested in this study. Various antagonists of proctolin receptors known from other systems could be used for further investigations. Proctolin analogues like [D-Tyr2, D-Leu3, D-Thr5]proctolin(Isberner, 2001) and many others (Konopinska et al., 1986a; 1986b; 1993; 1994; 1997; 1999; Kuczer et al., 1996; 1997; 1998; 1999) could be tested for whether they inhibit the proctolin-induced stridulation. Application of proctolin antibodies has also been used to inhibit proctolin-induced responses in cricket's antennal heart. Once effective antagonist and/or blocker of proctolin-induced stridulation can be found, the contribution of PRs activation on pharmacologically-stimulated stridulation can be examined. The latter will give an additional information about the interaction between proctolinergic and other metabotropic transmissions, contributing in the cephalic control of singing behavior in Ch.b.

\subsection{Methodological problems and optimization the stimulation} protocol to the proctolin mode of action - critical discussions

The methodic used in this study is very specific, not popular and has been used last 10 years developed by Ocker and co-workers (1995) for in vivo pharmacological studies. In the beginning only one-barrel microcapillary was used to test the pharmacological action of many transmitters and substances. These are the classical experiments carried out with this methodic and the problems touched in this chapter are not pointed to them. The problems have begun after the two- and in some cases three- chamber injection cappillaries have been used. Unfortunately the latter stimulations from two- or three-barrel capillary have shown many problems.

Since that time only a few control experiments were carried out but not systematically. Every methodic should have a specification and guide of use or at least a list with the possible problems, disadvantages and advantages. The first deepen work to study the setup itself, to describe the problems and to give criteria analyzing the results was Wenzel (2000) in her PhD Thesis, pp. 107 109. She has tried to describe and characterize the typical system errors, which 
could give system errors and should been kept in mind by the interpretation of the received data.

\section{Transition of injected substance into the other chamber}

Already in the control experiments using DMSO as well as in the later experiments with potentially inhibitory substances, in single cases it was observed that already the first injection pulse following muscraine injection caused singing behavior. This could be explained if some of the muscarine transited /went over/ diffused into the other chamber of the injection capillaries. In control experiments injecting into a drop of vaseline in fact after repeated injection of a colored solution the following drops of the originally clear solution was vaguely colored. This coloring was diminished with every on following drop and with the third drop not recognizable any more. To distinguish /declare any substance as initiating singing behavior, stricter criteria had top be met. Consequently, only substances were regarded as song initiating that consistently initiated singing behavior in at least three consequently injections (Wenzel, 2000).

The pressure-injected solution seems therefore to be pressed into the neighboring capillaries well, or to diffuse into it. Obviously it is found there in a lower concentration. This is in accordance with the observation that some substances are able to induce stridulation unexpectedly, but this was of significantly shorter duration than the previously muscarine-induiced stridulation. On the other hand, the solvent in the second chamber could be diluted by such a process. In experiments with potentially inhibitory substances the "inhibitory" effect could be due to a dilution of the afterwards injected muscarine-solution. But as the shortening of the muscarine induced song was not observed for all substances tested which did not by themselves cause stridulation, and as not all inhibitory experiments did show this effect, the effect of any potential dilution can be neglected (Wenzel, 2000).

The volume injected from both chambers could not be determined

A further problem with the pressure injection was that despite the use of defined pressures and pulse durations in the application of optimally identical volumes of solution from both chambers of the double capillaries as well as from the capillaries used in different experiments. In the beginning of any 
experiment the size of the resulting drop was adjusted to identical sizes by injection into the air, but which amount was released into the tissues could not be determined. The capillary can be blocked by haemolymph or by remains of tissue in part or in total. On the other hand, the end of the capillary might have been broken in the process of initial injection into the brain. Additionally to these small variations of applied volumes, the concentration of the substances used to get to the injected neuronal structures was significantly dependent on the distance as well as structure tissue between the spot of injection and the neurons. This is to caution that between different experiments as well as between two substances in one experiment the amount of active substances could not be presumed to be identical (Wenzel, 2000).

These problems have to be kept in mind then interpreting the results. Direct quantitative comparison between different experiments was not possible due to the problem of unspecified concentrations at the place of injection (Wenzel, 2000).

Summarizing the control experiments it could been concluded that they are not sufficient to have a reliable guide and specification of the methodic pharmacological stimulation into the brain of grasshoppers in vivo with two- or more chambers capillary.

One of the first aims of this study is to deepen the problematic described upper to test the methodic itself, to classify the problems, to find alternative protocols of injection a test substance preventing artificial results and misinterpretations.

For instance, to measure the concentration of the transitional substance in the next chamber after injection could be suitable the use of a capillary electrophoresis coupled to mass-spectrometry. In such manner the transition between the chambers could be quantified and control curves could be built for analyzing the results and this methodological disadvantage could be transformed to advantage and the method could become reliable and will reveal its potentialities.

The methodological problems mentioned here were basis of further investigations, described in section 3.3. 
In several control experiments (data not shown) with proctolin in one chamber of the injection capillary and Ringer solution in the other, Ringer solution, applied after several injections proctolin, could also elicit stridulation that was of shorter duration when compared to the proctolin-initiated singing. This means that a small portion of proctolin may have entered the chamber containing Ringer solution during previous ejections of proctolin and that was sufficient to induce stridulation in the pre-activated state of the control circuits. One criteria has been involved by Wenzel (2000) that a test substance that stimulates stridulation in 3 consecutive injections, after initiating stridulation through another excitatory substance (muscarine), could be considered as a substance that can initiate and maintain stridulation. In the present study this criteria was not used after the mentioned above control experiments were carried out. Than and than only one substance can initiate and maintain stridulation if injecting by one-barrel capillary, is capable to do this. The potential stimulatory effect of the test substance can be also proved using two-barrel capillary if the second chamber contains Ringer solution. Control injections into vaseline, as done by Wenzel to measure the transition between both chambers, should be made more accurately using the entire stimulation protocol (for instance 3-1-4) by different openings of the capillary tip and different pulse parameters. Several control experiments, with dye-stained solutions under visual observation, could not be sufficient to generalize that 3 injections are sufficient to clear the transited substance, because lower concentrations of the dye could not be seen. Under physiological conditions a small amount of a test substance introduced to the next chamber, in $\mu \mathrm{M}$ concentrations, would be visually undetectable, may significantly affect the responses. Furthermore, the dilution of the control (excitatory) substance after pulses from the other chamber and not inhibitory pharmacological effect could alone decrease the duration of stridulation. The latter could lead to misinterpretations of the received data if the response (song parameters - latency and duration) is critically dependent on the concentration, or the volume under our stimulation conditions.

The results in section 3.2.4 showed that alterations in the limits of $20 \%$ for the durations and up to $50 \%$ increase of the latency could owe to dose effect. If these alterations are higher than this values it could be observed as pharmacological effect. Concerning the "all/none" responses received by smaller amount proctolin injected, the latter criteria should be not applicable if 
no response occurs. In case of "completely inhibition" occurred along the experiment the entire stimulation protocol was repeated using 1,5X pulse duration. If the none-responses occurred again, than only the result was included in further evaluations.

In the present study another stimulation protocol 3-3-3 was suggested, that solves some of the problems described (section 3.3). Instead of filling the second chamber with a test substance (B) (Fig. 4-3, left) a mixture of the excitatory and the test substance $(A+B)$ was included. Thus, the concentration of the excitatory substance (A) (either proctolin or muscarine), in both chambers is the same. In the case of transition between the two chambers only the concentration of the test substance would be altered with the repetitive injection from both chambers. Dilution and any alterations of the concentration of the excitatory substance resulting from injections from the other chamber is prevented througout the entire experiment.
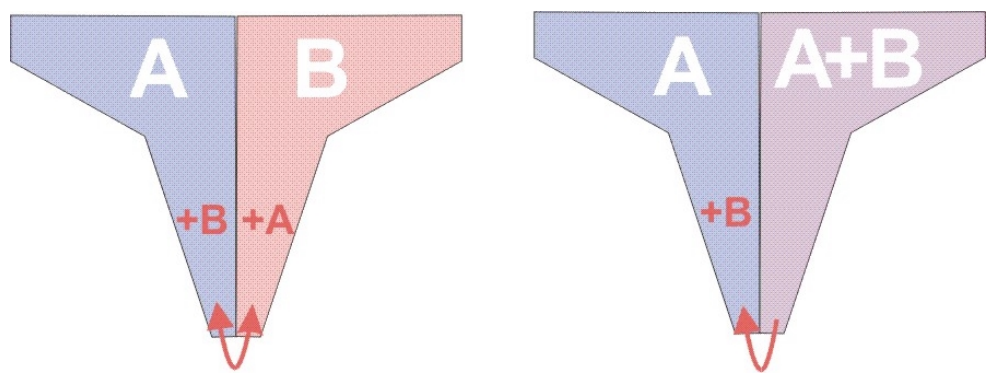

Fig. 4-3 Comparison between protocol 3-1-4 (left) and protocol 33-3 (right), using two-barrel capillary.

A - excitatory substance (proctolin, muscarine etc); B - test substance The red arrows show the transition of solution between the chambers during ongoing injection.

\subsection{Additional remarks on the pharmacological studies}

The activity of proctolin (obtained from Sigma) depends on following factors. Proctolin should be diluted in $\mathrm{dH}_{2} \mathrm{O}$ and preserved at $-20^{\circ} \mathrm{C}$ for no longer than 3 months. The maximal concentration of proctolin in the stock solutions can be $10 \mathrm{mM}$. To prepare a $1 \mathrm{mM}$ concentrated solution proctolin or a solution of proctolin in combination with another neuroactive substance, a concentrated Ringer solution (5X) has to be used, preserved without sucrose and $\mathrm{CaCl}_{2}$. For the latter two Ringer compositions 20X or even 50X stock solutions are recommended. Freshly prepared proctolin Ringer solutions can be used for as long as 3 days. Otherwise the induced stridulation becomes 
unstable, and stimulation of stridulation gets harder to accomplish, indicating that proctolin loses excitatory potency when kept in solution for longer periods. Dopamine, ACh, histamine, glycine and the other natural transmitters should be used within 1 to 3 days after dissolvence. Ringer solution (5X) without sucrose and $\mathrm{CaCl}_{2}$ should be made at least every month. Drugs diluted in Ringer solution older than 1 month may lead to unexpected experimental results, such as prolongation or decrease of proctolin- or muscarine-stimulated stridulation even when the freshly made solution of a particular substance may show the opposite effect. Since many bacterial toxins are used as selective activators activators of Gq proteins, that in turn activate PLC signaling pathway, mediating both proctolin- and muscarine-stimulated stridulation, the altered effects of old drugs in Ringer solutions may be induced by bacterial growth and accumulation of toxins, acing at $\mathrm{nM}-\mu \mathrm{M}$ concentrations. In one control experiment old (more than 6 months) vs. fresh Ringer solution was tested. The old saline reliably and robust initiated stridulation in O.v. and initiated only once stridulation in Ch.b. The fresh saline failed to initiate stridulation in both species.

The effect of a potential bacterial toxin in old Ringer solutions may multiply in the presence of an additional excitatory substance. This appeared in early experiments examining the potential effect of inhibitory substances, such as W7, sphingosine (PKC inhibitors) in very old Ringer solutions. Fresh prepared showed the opposite effects. It will be interesting to identify those bacteria, which produce the toxins capable of triggering singing in grasshoppers and to isolate this toxin.

All these observations clearly indicate that the accurate conductance of the pharmacological experiments requires sterile conditions and the use of freshly prepared solutions. 


\subsection{Short introduction to the physiology of central nervous neuronal} networks

Neurons, as pointed out by Llinas (1988), are not interchangeable; i.e. a neuron cannot be functionally replaced by one of another type even if their synaptic connectivity, type of afferent neurotransmitters and receptors to these transmitters are identical. The activity of a neuronal network is related not only to the excitatory and inhibitory interactions among neurons but also to their intrinsic electrical properties as well. The "personality" of a neuron is defined by its input-output characteristic; i.e. its firing pattern (output) in response to a depolarization or a hyperpolarization (input).

Input-output characteristics are the result of a rich repertoire of ionic currents other than those of the action potentials. These currents, inward or outward, are called subliminal voltage-gated currents, because they are activated at voltages sub-threshold to that of action potentials (supraliminal currents) (Hammond, 2001). They are located either in the dendritic or the somatic membrane or both. The subliminal currents, which can either depolarize or hyperpolarize in different time courses the membrane under the threshold. Depending on their location, subliminal currents are activated by different signals. When located in dendrites, they are activated by a depolarization (EPSP) or a hyperpolarization (IPSP) of synaptic origin. When located in the soma-initial segment membrane, they are activated by the first action potential generated or by the hyperpolarization that follows an AP (after spike hyperpolarization). Depending on their location, subliminal voltage-gated currents also have different roles. When present in the dendritic membrane they boost or counteract EPSPs or IPSPS, but when present in the soma-initial segment membrane they underlie intrinsic firing patterns, modulate synaptic driven firing patterns or participate in network oscillations. When present in the whole neuronal membrane, subliminal currents that are activated around rest and that do not rapidly inactive $\left[I_{h}, I_{M}\left(K^{+}\right.\right.$current sensitive to muscarine $), I_{K I R}$ (inward $\mathrm{K}^{+}$rectifier)] also determine resting membrane potential, resulting to increasing or decreasing the excitability of the neuron at rest (Hammond, 2001). Many subliminal voltage-gated currents are effectors of different divergent and convergent second messengers pathways. They modulate the subliminal 
currents by opening, closure, of the respective channels or by altering the time course of their activity.

The integration of the inputs and outputs (supraliminal currents) of all neurons of a single neuronal network characterize the input/output patterns of this network, for instance, one central pattern generator. All intrinsic properties (all subliminal currents) of all single neurons represent the intrinsic properties of the generator, as well. The subliminal currents are targets of a fine tuned modulation through large family membrane receptors, called metabotropic (mAChRs, PRs, mGluRs, mGABA ${ }_{B}$, Dopamine-Rs etc.). They are coupled to Gproteins, which activation triggers a cascade of membrane or cytoplasmic biochemical processes, involving second messengers. The effectors of these intracellular cascades or signal pathways (signal transduction cascades) can be membrane channels, conducting supra- or subliminal currents. This intracellular signal transduction via metabotropic occurs parallel to the conventional mode of neuronal action via the voltage-gated and/or ionotropic ligand-gated membrane channels of the neuron and thus can significant influence the firing patterns. This modulation is short-term, for instance, if the effectors of the triggered second messenger pathways are membrane channels, that participate in the firing pattern generation and long-term, for example, if the effectors of the informational flow are genes regulating the expression and localization (dendrites, soma or soma-initial segment) of the channels, processing supraand subliminal currents.

Fig. 4-4 illustrates the information flow initiated by metabotropic receptors, coupled to G-proteins and relevant second messenger cascades, and their effectors - channels, conducting subliminal currents. The sensing and processing of the stimuli are mediated by signal transduction cascades in a single neuron. These molecular circuits in neurons are constructed from: Receptors, Enzimes, Channels, Regulatory proteins. They detect, amplify and integrate diverse external signals.

In order to demonstrate the complexity of the intracellular signal transduction, one classical example - the activation of mAChRs - will be presented. 


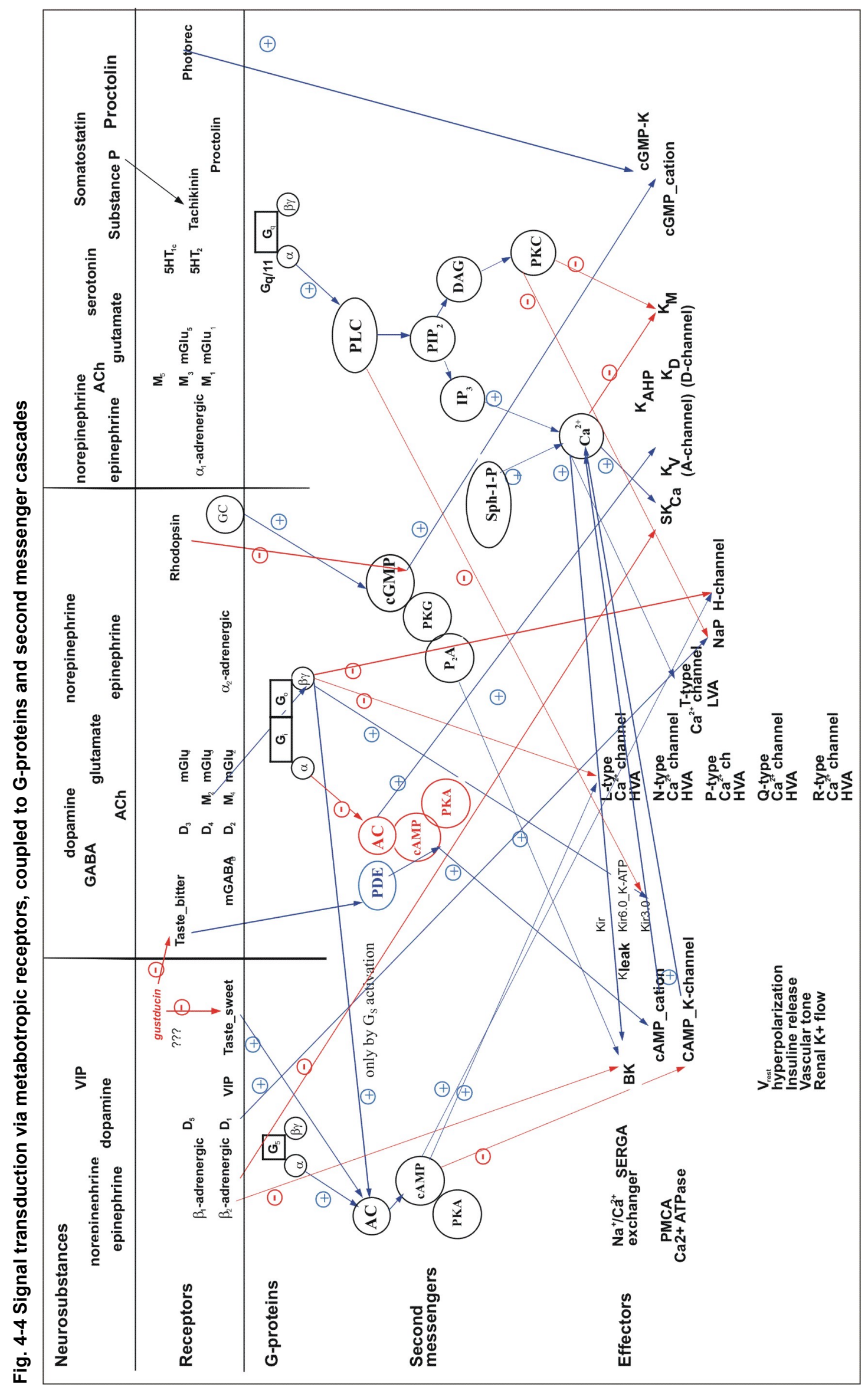


In vertebrates five types of mAChRs $\left(M_{1}, M_{2}, M_{3}, M_{4}, M_{5}\right)$ have been identified and cloned to date. Each type trigger different signal transduction pathway, depending on its coupling to particular second messenger pathway. $M_{2}$ and $M_{4}$ types ( $M_{2}$-like) lower the cytosolic level of cAMP, when localized presynaptically and coupled to the inhibitory $\mathrm{Gi}$ and $\mathrm{Gi} / \mathrm{o}$ protein through its active a subunits [when activated $\mathrm{G}_{\mathrm{s}}$ protein hydrolyzes (GTP-depending) to a and $\beta y$ subunits, which both trigger divergent processes](Fig. 4-4, in the middle). cAMP can activate PKA, which in turn phosphorylates (activation or modulation) channels, conducting supra or subliminal currents. Thus the level of PKA activity, in case of $M_{2}, M_{4}$ activation, is lowered (low concentration of cAMP). The equilibrium is shifted towards dephosphorylates states of the channels trough ongoing activity of phosphatases, which oppose the PKA effects. On the other hand, $\beta$-subunit of the inhibitory $\mathrm{Gi}$ and $\mathrm{Gi} / \mathrm{o}$ protein can interact directly (without second messengers) with a $\mathrm{K}^{+}$channel, conducting another subliminal current. Thus the $\mathrm{Gi}$ and $\mathrm{Gi} / \mathrm{o}$ proteins, through their $\alpha$ and By subunits, could transform an extracellular stimulus, which activates one receptor, into a bifurcating signal, finally affecting different ion channels. The latter is a main feature of signal transduction cascades in general. The molecular circuit triggered by $G_{q / 11}$ proteins coupled to $M_{1}, M_{2}$ and $M_{3}$ receptors $\left(\mathrm{M}_{1}\right.$-like) consists of two branches after activation of PLC. $\mathrm{IP}_{3}$ and DAG are second messengers, which act on different targets, increasing of intracellular $\mathrm{Ca}^{2+}$ level and activation of PKC, respectively. The classical example of localized postsynaptically M1-like receptors initiated effects, is modulation of potassium channels, conducting the so called M-current ( $I_{M}$ subliminal current, sensitive to muscarine). Thus the $\mathrm{K}^{+}$conductance of the membrane is lowered, which in turn leads to a slow membrane depolarization. This current differs from other subliminal depolarizing currents because it does not inactivate (Hammond, 2001). A slow activation and a lack of inactivation of the M-current leads to a prolonged excitation of the membrane. The spiking threshold will be easier reached by excitatory synaptic inputs and the same excitation will result in a stronger response of the neuron (stronger EPSPs and high frequency spiking).

One of the main features of the intracellular signal transduction is that the pathways can diverge, they bifurcate and built tree structure of the informational 
flow within the cell. One receptor type can modulate many effectors and targets as it was be demonstrated above.

The first prerequisite of the bifurcation of the triggered cascade is the $G$ protein hydrolization to $\alpha$ and $\beta y$ subunits. While the $\alpha$ subunits of $\mathrm{Gi}$ and $\mathrm{Gi} / \mathrm{o}$ or $\mathrm{Gq}$ couple mAChRs to different cellular effector systems, the $\beta \gamma$ subunits of the $G$ proteins may also have a role in mediating the cellular effects of muscarinic agonists. It has been reported that $\beta y$ subunits of $G$ proteins can mediate muscarinic stimulation of $\mathrm{K}^{+}$channel activity in cardiac cells. The effect has been postulated to involve the $\beta y$ subunits dissociating from the a subunits of $\mathrm{Gi}$ or $\mathrm{Go}$ following agonist stimulation. The free $\beta y$ subunits have been proposed to stimulate phospholipase $A_{2}$ activity to increase arachidonic acid (AA) production. The $A A$ is believed to then stimulate the $\mathrm{K}^{+}$channel activity directly (Fukuda et al., 1987). The finding is of interest since it reveals a function of the $\beta y$ subunits independent of the a subunits.

Another intrinsic property of the signal transduction is that different second messenger pathways, triggered by activation of different receptors, can modulate the same effector but not always in opposite manner. They are called convergent pathways. Various examples of divergence and convergence of signal transduction pathways are included in Fig. 4-4.

In addition, different signaling pathways or their bifurcating limbs can be activated simultaneously and with interact each other in various ways. The cross-talk between the pathways can be homologous (within a given cascade) and heterologous (two cascades, triggered at the same time).

Typical example for positive homologous interaction is the PLC pathway that uses two second messengers - IP 3 and DAG. PKC activated through DAG, requires additional free $\mathrm{Ca}^{2+}$ level for its activation. $\mathrm{Ca}^{2+}$ is released either by $\mathrm{IP}_{3}$-gated $\mathrm{Ca}^{2+}$-channels on the membranes of intracellular $\mathrm{Ca}^{2+}$-stores or by opening of $\mathrm{Ca}^{2+}$-channels on the neuron's membrane. Such feedback homologous interactions between second messenger pathways may be the basis of the oscilatory phenomena that are being uncovered in many cell types.

The level of cAMP can be regulated by heterologous interaction between two main signalling pathways induced respectively by $\mathrm{Ga}$ (activates $\mathrm{AC}$ ) and $\mathrm{Gi}$ 
and $\mathrm{Gi} / \mathrm{o}$ (inhibits $\mathrm{AC}$ ) proteins. The net effect of this cross-talk will determine the level of cAMP and its fluctuations in the time.

Finally the second messenger levels oscillate in many cell types. That seems to be dependent on the receptor type, which trigger the cascade. For instance, $\mathrm{M}_{3}$ and $\mathrm{mGluR}(5)$ receptors, via PLC activation, induce $\mathrm{Ca}^{2+}$ oscillations of different time courses in the same cell (Nash, 2000). Oscillation in intracellular $\mathrm{Ca}^{2+}$ concentration induced by GPCRs in the membrane provide a versatile encoding mechanism that uses variations in the amplitude, frequency and duration of signals to control cellular processes (Berridge et.al., 2000; DeKonink et.al., 1998; Dolmetsch et.al., 1998). Models to explain these oscillations are broadly based on dynamic uncoupling of the $\mathrm{PLC} / \mathrm{IP}_{3}$ signalling pathway, or on the self-propagating regulatory properties of $\mathrm{Ca}^{2+}$ on the $\mathrm{IP}_{3}$ receptor (known as $\mathrm{Ca}^{2+}$-induced $\mathrm{Ca}^{2+}$ release) (Taylor and Thorn, 2001; Harotunian et.al., 1991). Distinction between the two schemes relies on whether $\mathrm{IP}_{3}$ oscillations, from repetitively switching phospholipase $\mathrm{C}$ on and off, are the driving force, or whether $\mathrm{Ca}^{2+}$ alone controls this process by enhancing or inhibiting its own release from internal stores at low and high concentrations, respectively (Nash, 2001).

The ability of a single neuron to encode a large content of information via its intrinsic properties, including the signal transduction cascades, triggered by metabotropic G-protein coupled receptors enables it to be a complicated integration center (in contrast to the classical cable model), which is capable to act and react properly to multitude of different situations and to the actual state of activity within the network. The complexity of the molecular circuit of the signal transduction with its many interactions, a fine-tuned modulation at every level of processing (receptor, G-proteins, second messengers, effectors) and positive and negative feedbacks loops, matches with the complexity of entire network. Furthermore, many neuronal projection fibers and thus many axondendrite, axon-axon and dendrite-dendrite connections may also contribute to information processing within a particular network.

With the present knowledge the complexity of the cephalic network controlling grasshopper stridulation can only be estimated. The local injections of neuroactive substances used in the present approach are not sufficient to 
conclude details about the functions of individual neurons contained in this circuit.

On the other hand, modulation of the general activity of the network or circuit of neurons through a local release of hormone and modulators could partly be mimicked. The accumulation of excitation through repetitive injections of proctolin and muscarine may be similar to the mode of actions of one local neurohormone. The presence of numerous neuropeptides in the pars intercerebralis and central complex, besides proctolin suggests the release and co-release of number of neuropeptides, which can alter the activity of sets of neurons that control sound production in Ch.b.

It is obvious that the pharmacological approach of injecting neuroactive substances into particular brain areas or intracellular recordings of single neurons, is not sufficient to reveal the intrinsic properties underlying the activity of the entire neuronal network.

Therefore, to comprehend information about the network itself, which is situated presynaptic to the command neurons directly controlling the singing behavior in Ch.b., this pharmacological approach has to be combined with behavioral, electrophysiological, immunohistochemical and molecular genetic methods to reveal how sensory information is integrated through the intrinsic properties of the network to mediate the activation of the most appropriate motor program in a particular situation.

Nevertheless in vivo pharmacological studies on the control system of the acoustic communication in Ch.b., can be used to collect very important data, about the net effects of a particular neurotransmitter, neuromodulator or local neurohormone or their combined mode of action on the activity of the decision making network - when, what and how to sing. Such data, for instance, could not be received through electrophysiological in vivo experiments aiming to study only the outputs of a single neuron, which is inseparable from the whole neuronal network activity. The advantages of the present approach are discussed further. 
4.11 Second messenger signaling coupled to proctolin-stimulated

\section{singing}

\subsubsection{The AC pathway}

It has been shown that inhibition of $\mathrm{AC}$ decreases the singing responses stimulated by muscarine in Ch.b. (Wenzel et al., 2002). Moreover, Wenzel $(2000,2002)$ has shown that an increase of cAMP level can initiate alone stridulation after once the basal excitation is increased through previous mAChRs activation.

Since, the direct coupling of mAChRs to AC pathway is uncommon the involvement of indirect effects could not be excluded. The increase of cAMP level is also coupled to neuropeptide receptors in many systems (Lundquist and Nässel, 1997; Nässel, 2002). Likewise, proctolin has been reported to increase the cAMP level in the brain of Locusta migratoria (Hiripi et al., 1979). An inhibitor of AC (SQ22536) decreases both muscarine- (Wenzel, 2002) and proctolin- (section 3.5.1.1) stimulated stridulation in Ch.b. But, in this species injection of $\mathrm{ACh}$, or $\mathrm{ACh}$ and eserine, failed to mimick the muscarinic prolonged effect (Hoffmann, 2003).

Possible co-realesed of proctolin can induce species-specific song and may mimick the mAChRs-activation through muscarine in Ch.b. In addition, the activation of mAChRs is not necessary for proctolin-stimulated stridulation (section 3.4.5) Thus, suggests that PRs and not mAChRs could be coupled to cAMP signaling in Ch.b. Because the pharmacological actions of both muscarine and proctolin are positive interconnected decreasing the cAMP level most likely coupled to PRs can affect indirect the muscarinic-induced singing. On the other hand elevation of CAMP could trigger stridulation, if once the basal excitation is established through mAChRs activation (Wenzel, 2002), can be addressed also to indirect positive effect through proctolin-mediated excitation.

Another interesting possibility to explain the involvemnt of cAMP in the control of singing behavior in Ch.b. could be a heterologous interaction between two different signal pathways that both controlling the cAMP level. In contrast to the direct inhibition of $A C$ through $\mathrm{Gi} / \mathrm{Go}$ proteins mAChRs $\left(\mathrm{M}_{2,4}\right)$ and $G_{A B A_{B}}$ receptors can also have another seemingly opposite effect on 
CAMP accumulation. When AC is stimulated to produce cAMP by Gs protein-coupled receptors [neuropeptides, norepinephrine, dopamine $\left(D_{1}\right.$ like receptors), adenosine $\left(A_{2}\right.$ receptors)], $\operatorname{mAChR}\left(M_{2,4}\right)$ or $G_{A B A}$ receptor activation will enhance this increase in cAMP accumulation by two or three fold (Karbon et al., 1984). This effect is contrary to the inhibition of $A C$ discussed above. The mechanism of this effect lies in the ability of the $\beta \gamma$-subunit from Gi/Go protein, liberated by the activation of $M_{2,4}$ or $G A B A_{B}$ receptor, to synergize the interaction of the $\alpha_{S}$-subunit of the $G_{S}$ protein with certain types of $A C$. These $\beta \gamma$-subunits can come from either $\mathrm{G}_{S}$ or $\mathrm{Gi} / \mathrm{o}$ proteins. In this way $A C$ can act as a molecular "coincidence detector" by responding minimally to activation by a single signal but synergistically to the coincident arrival of dual signals through separate pathways (Hammond, 2001).

This idea deserves attention and further investigations, since it offers another mechanism for the coupling of $\mathrm{mAChRs}$ to $\mathrm{AC}$ signaling.

\subsubsection{The PLC pathway}

The blockade of PLC through neomycine and $\mathrm{Li}^{+}$showed stronger overall inhibitory effect on the proctolin-stimulated stridulation than the inhibition of AC, since complete inhibition was common in most of the experiments (section 3.5.2.1; 3.5.2.2). This suggests that the PLC pathway plays a main role in both phases - initiation and maintenance of the stridulation. Initiation of stridulation is used as triggering the stridulatory activity, and maintenance - prolongation of the duration, leading to performance of entire song sequences of $2^{\text {nd }}$ order. Partly decrease of the song durations through inhibition of the AC pathway indicates that it may play a role mostly in the maintenance phase, increasing additionally the proctolin-stimulated singing.

PRs that stimulate singing in Ch.b. are coupled to PLC signaling pathway and most likely use DAG and $\mathrm{Ca}^{2+}$ as second messengers. The accumulation of intracellular $\mathrm{Ca}^{2+}$ (or $\mathrm{Ca}^{2+}$ oscillations) ows to gating of membrane $\mathrm{Ca}^{2+}$ channels (blocked by $\mathrm{Ni}^{2+}$ ) (section 3.5.2.3) and $\mathrm{IP}_{3}$-insensitive $\mathrm{Ca}^{2+}$-release from the intracellular stores (section 3.5.2.3). $\mathrm{Ca}^{2+}$-dependent $\mathrm{Ca}^{2+}$ release could not be excluded. Both, the increase of intracellular $\mathrm{Ca}^{2+}$ levels (through 
thapsigargin) and PKC activation (through phorbol ester) potentiate proctolinstimulated responses, indicating additional level of excitation to this induced by proctolin. This may suggest a potential side entry for additional synergistic effects on singing related arousal from other receptors coupled to PLC signaling pathway. Moreover, phorbol ester injected alone to sites, where proctolin was induced singing, mimicked partly the proctolin response.

mGluRs $(1,5)$ activation (DHPG) coupled to PLC signal pathway stimulates singing similar to the proctolin-induced one, but glutamate alone triggers only precopulatory and not stridulatory movements. Gluatamate co-released with proctolin modulates the stridulatory movements and enhances the duration of the song.

These data suggest that the PLC pathway is target to multiple modulatory inputs triggered by at least PRs, mAChRs and mGluRs.

Studies on the role of mAChRs and PRs in foregut muscle contraction of Schistocerca gregaria have revealed receptor-specific activation of the PLC signaling cascade (Hinton et al., 1998). Stimulation of mAChRs receptors caused activation of PKC, thereby promoting $\mathrm{Ca}^{2+}$ entry via the opening of $\mathrm{L}$ type $\mathrm{Ca}^{2+}$ channels (verampil-sensitive). In contrast, activation of PRs caused a verapamil-insensitive entry of extracellular $\mathrm{Ca}^{2+}$ as well as the generation of $\mathrm{IP}_{3}$, leading to the release of $\mathrm{Ca}^{2+}$ from intracellular stores to bring about an increase in gut contractility (Hinton et al., 1998).

A similar receptor-specific activation of the PLC signaling cascade cannot be excluded in the song control system of Ch.b.

$\mathrm{K}^{+}$and $\mathrm{Ca}^{2+}$ channels are candidate effectors of PRs, respectively of their second messenger cascades, that could mediate the initiation and maintenance of proctolin-induced stridulation.

\subsubsection{Conclusions about the organization of the second messenger} pathways, involved in proctolin-stimulated stridulation in Ch.b.

Previously, a consecutive activation of two signal pathways has been suggested to mediate the muscarine-stimulated singing behavior in O.v. and Ch.b. (Wenzel et al., 2002). 
Stridulation that was stimulated by direct activation of adenylate cyclase through injection of forskoline could be suppressed by application of neomycine, which inhibits the PLC pathway (Wenzel and Heinrich, unpublished). This suggests a heterologous interaction between both pathways. Since proctolin frequently found to be co-expressed with $A C h$, glutamate, GABA in the periphery, possible co-expression of PRs with mAChRs and mGluR(1,5) on the same neurons may also occur. Whether such a co-expression may exist in the grasshopper brain has not been studied and cannot be determined with the method used in this study. Information related to this question may arise from cell culture experiments, where direct effects of agonists on a particular neuron and expression profiles of its pharmacological receptors can be determined.

PRs, as well as mAChRs, most likely coupled to AC/cAMP pathway and PRs, mAChRs and mGluRs $(1,5)$ coupled to PLC pathway are involved in the control of the singing behavior in Ch.b. Co-activation of these types of receptors can control the initiation and maintenance of stridulation and may induce the transition between different song types. Since different mechanisms can be expected to control the initiation and the maintenance the role of the receptors and their signaling cascades could have different mode of action in both phases of stridulation. The latter is valid also to the transition from calling to courtship song. Interpreting the role of the examined receptors activation and the relevant signaling cascades should be considered in relation to the different phases of the courtship in Ch.b. The received results support such idea, since the responses to co-aplication of proctolin/muscarine, proctolin/eserine (nAChRs), proctolin/dTC (nAChRs), proctolin/glutamate induced mixed singing patterns.

The courtship behavior (in general sense) of Ch.b. from pharmacological point of view consists:

1. Initiation calling song

2. Maintenance calling song

3. Transition to courtship song

a. recognition of species- and sex-specific song

b. initiation of courtship song

4. Phonotactic mate approach 

a. maintenance of courtship song
b. oriantation and phonotaxis
c. a) and b) alternate

\section{5. copulatory act}

Since the data received in this work are not sufficient to cover such overview on the entire courtship only indications that the activation of particular receptors may play a different role in particular stage of the courtship will be shown and discussed in the next sections.

\subsection{Modulation of singing behavior through co-transmission in the brain of Ch.b. (the role of proctolin)}

A number of immunocytochemical studies have suggested that, as in vertebrates, co-localization of different transmitters in the same neurons is a common phenomenon in insects. However physiological studies on cotransmission in the insect brain have not been yet performed (Homberg, 1994; Nässel, 2002). Co-localization of peptides or other transmitters with ACh, GABA and glutamate has not been investigated in the insect brain (Homberg, 1994) with a few exceptions (glutamate and proctolin control antennal muscles contractions).

The release of several transmitters dramatically increases the possibilities of neuronal interactions in the nervous system. A fast acting classical transmitter can often be distinguished from a slower acting secondary transmitter, which is often released only at higher discharge rates. This second co-transmitter is in many cases a peptide (Homberg, 1994; Nässel, 2002). A variety of antagonistic, cooperative and seemingly unrelated effects have been described for co-release of transmitters, and no general rules have emerged so far (Kupfermann, 1991, Weiss et al., 1992, Hinton et al., 1997). Cooperative effects of peptide/peptide and peptide/transmitter interaction might be expected for the accessory medulla and central complex in insects (Homberg, 1994).

The singing behavior of male Ch.b. is under strict control of the brain. In the present study it was clearly demonstrated that complex modulation of the singing parameters of stridulation (latency, duration) and the performed singing patterns (the duration of the single sequences amplitude of the stridulatory 
movements (Heinrich et al., 2001), also precopulatory movements and wings flapping (section 3.6.4) takes place in the brain.

ACh through mAChRs may stimulate calling song in Ch.b. Proctolin as neuromodulator, most likely co-released may play a role in switching to courtship song and the associated behaviors. The fine modulation of the singing patterns during the perception for mating seems to be realized through processes of co-release and simultaneous release of transmitters and modulators into the neuronal control circuits presynaptic to the command neurons. The present study provides many indicationsthat support this hypotesis.

4.12.1 Singing patterns I and II of the normal song (calling and courtship)

The production of singing patterns I and II has been demonstrated to occur in the $3^{\text {rd }}$ thoracic ganglion, where the central pattern generators are located. Many studies have reported a lost of phase differences between the movements performed by both HLs. After removal of one leg, the remaining leg exclusively performs pattern I. All these data suggest that pattern and phase differences originate in the thoracic CPGs and interactions between them.

Proctolin, as well as muscarine (Wenzel, 2000) can trigger alternations of both patterns between the HLs only in different sequences, similar to the natural song. In a few experiments the singing patterns (I and II) changed between the HLs within a particular sequence of $1^{\text {st }}$ order after stimulation with the mGluRs(1,5) activator, DHPG (Fig. 3-71, B). This suggests a role of the brain in the modulation of the performance of both singing patterns.

\subsubsection{Uncoupling of the basal excitation to sing from the stridulation} itself

In many experiments was clear demonstrated that the latency of the singing can be shifted in time without decreasing of the level of excitation, the duration of the delayed response remains the same. This is a clear indication of a mechanism, underlying uncoupling of the basal excitation from the stridulation itself. Appropriate behavior, which could be walking, jumping etc. could not be obligatory associated with a decrease of the excitation of the stridulation controlling system. Under natural conditions the male during the singing walks, 
jumps, active looks for the female and immediately reactivates the stridulation. Such uncoupling of the basal excitation from the singing itself seems to be the main role of the brain, integrating the sensory information and the motivation of courting. The arousal of the basal excitation is a slow process in the range of minutes. Both muscarinic and proctolinic excitation lasts at least 5-10 min in Ch.b. Every locomotor activity, driven by the HLs is rapid in the range of msec and sec. The uncoupling (shifted in time) of the basal excitation from other than stridulatory locomotor HLs movements would be also a very effective mechanism in the repertoire of the decision-making brain center, controlling the phonotactical mating approach (Fig. 3-3).

Alteration of the latency but not of the duration of the singing stimulated by proctolin suggests a brain mechanism that control initiation of the stridulation when to sing. According to the data received in the present work is hypothesized that the co-release of proctolin and modulatory processes, mediating by metabotropic receptors in Ch.b. brain may cause uncoupling of the basal excitation from the stridulation itself (control of the initiation of stridulation - when to sing).

On the other hand during the calling a prolonged excitation should be triggered by abiotic factors (temperature, light etc.). mAChRs activation may

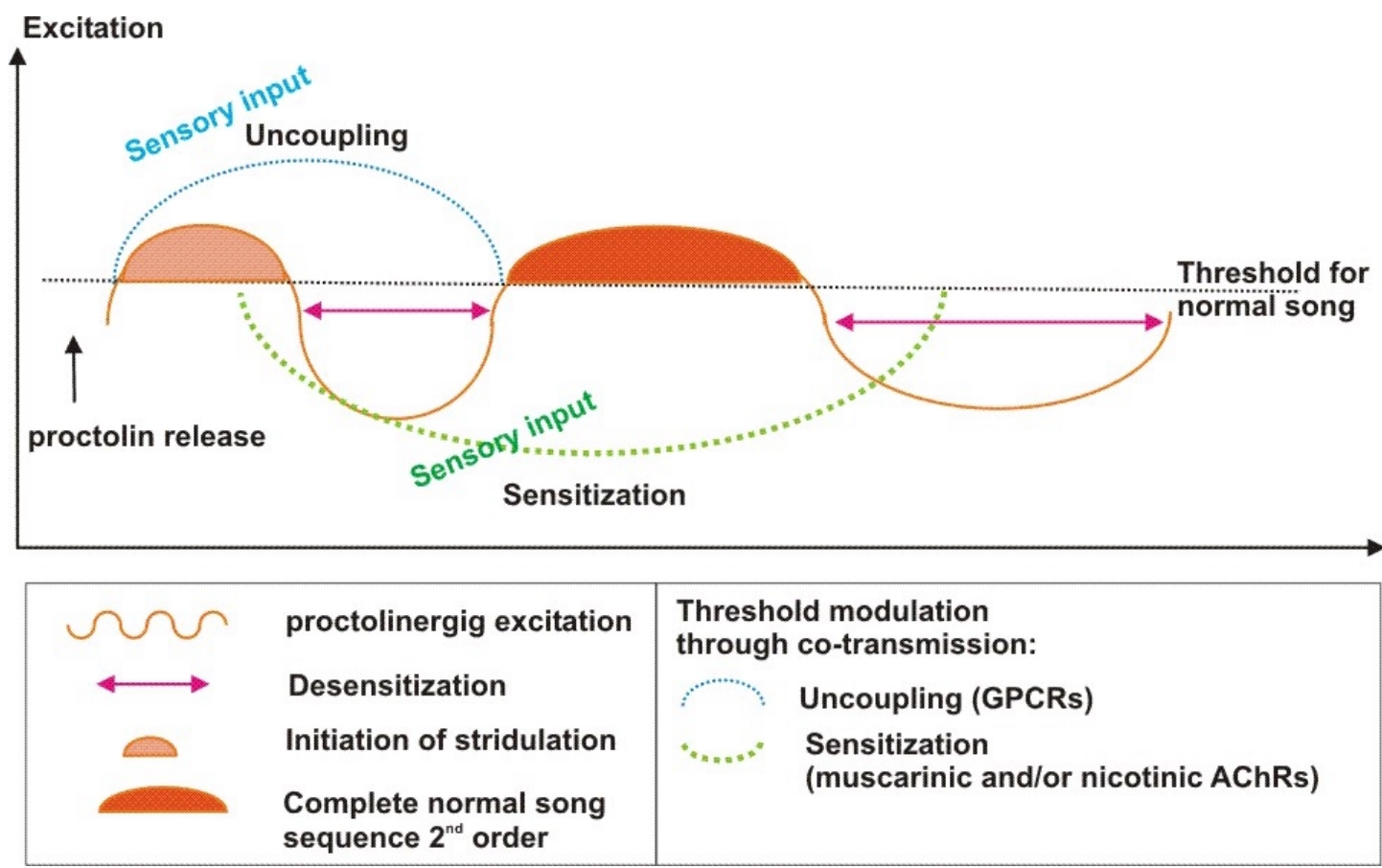

Fig. 4-5 Threshold modulation through co-transmission:

Uncoupling of the basal excitation from the stridulatory activity may play a role in the control of the initiation of stridulatory in the phonotactical mate approach in Ch.b. - when to sing.

Sensitization during the calling - abiotic factors (temperature and light) - how long to sing. 
play this role by lowering the threshold of the command neurons sensitized them (control of the maintenance of stridulation - how long to sing) (Fig. 4$5)$.

\subsubsection{Conclusions on the role of proctolin in the courtship of Ch.b.}

The calling song, induced by muscarine, can transform into courtship song in presence of an additional excitatory stimuli from the brain auditory neuropil that recognizes the con-specific female song. Co-release of proctolin can alter the duration of the sequences of $1^{\text {st }}$ order and triggering the courting. The results here suggest that the a second level of excitation, previously proposed by Heinrich and co-workers (2001) for the occurrence of stridulatory patterns associated with high courtship intensity in O.v., may be triggered by co-release of proctolin or other neuropeptides in Ch.b. brain.

The courtship (in general sense) of Ch.b. includes at least 3 complex behaviors:

1. Basal motivation to sing dependent mainly from abiotic factors temperature, light etc. (calling song)

2. Perception and recognition of the species- and sex-specific song pattern (Lautschema) (Fig. 3-4 and section 3.2.1)

3. Phonotactical mate approach that includes - courtship song, orientation (visual and spatial processing) and locomotor programs (jumping, walking, wing movements) (Fig. 3-3)

The transition between behaviors 1 and 3 is controlled most likely by 2 , an extremely strong stimulus for the male Ch.b. (von Helversen, 1997). Proctolin and probably other neuropeptides may be involved in this transition.

During the $3^{\text {rd }}$ behavior many brain structures and networks should be activated and activities coordinated. Thus, simultaneous release of other transmitters (ACh, dopamine, glutamate, etc.) can alternate the courtship song with other courtship acts, such as laterization, precopulatory movements and wing movements by jumping in a step-wise manner. At the same time the previously accumulated level of excitation, is maintained in the background, to assure the stridulation could immediately continue, as soon as the other behavior is terminated. 
Proctolin may play a role in such maintenance of background excitation induced in $2^{\text {nd }}$ and during the $3^{\text {rd }}$ behavior. Present pharmacological work suggests such a central nervous role for proctolin.

\subsection{Leg stridulation and wing flapping in Ch.b. and Stenobothrus} rubicundus

The wing stridulation and wing flapping occurs only in species with complex stridulatory pattern and rapid stridulatory movements $\left(50-70 \mathrm{sec}^{-1}\right)$ as seen in Ch.b. (section 3.6.4) and Stenobothrus rubicundus (Elsner and Wasser, 1995; Heinrich and Elsner, 1997).

For rapid vibratory movements $\left(50-70 \mathrm{sec}^{-1}\right)$, such as occur in the songs of Ch. mollis, Ch. biguttulus, Ch. albomarginatus, G. rufus, Myrmeleotettix maculatus and other species, it has been postulated that they may derived from flight patterns, which undoubtedly are phylogenetically older than hindleg stridulation (Elsner, 1983). This hypothesis is supported not only by the similarity in the movement frequencies but also by the bifunctionality of the pterothoracic muscles and the motoneurons that drive them. In the grasshopper Stenobothrus rubicundus it has been shown that simple changes in the coordination of muscle activation can convert the movement patterns typical of legs and wings into one another (Elsner and Wasser, 1995a, b; Schütze, 1999). The pattern of neuronal excitation underlying flight is generated by a thoracic network (Robertson and Pearson 1983, 1985). It appears that to coordinate the wings, as in the case of the rapid stridulatory movements of the hindlegs, no cross-connections are used apart from the pathways in the pterothoracic ganglia. Indeed, even the mesothoracic commissures are not obligatory for wing coordination, since longitudinal transection of the mesothoracic ganglion does not affect the coordination of neither the forewings nor the hindwings. The coordination is disturbed, however, when the metathoracic ganglion is split (Ronacher et al., 1988; Wolf et al., 1988). Here a neuronal mechanism has been evolved that might also be utilized to coordinate the rapid singing movements of the right and left hindlegs. 


\subsubsection{Wing stridulation in Stenobothrus rubicundus}

Males of the gomphocerine grasshopper species Stenobothrus rubicundus (GERMAR 1817) produce calling and courtship songs by rubbing their hindlegs against the folded forewings, like other related species. The males do not become active until the temperature exceeds $30^{\circ} \mathrm{C}$. Additionally, they show brief sequences of short sound pulses in these songs. These pulses originate from a step-wise downstroke of the hindlegs in a population found in Alps, Slovenia and Croatia, but is produced by wing movements with the same frequency, while thickened veins of the hind wings touch each other at the upper reversal point, in a population in Greece (Elsner and Wasser, 1995). This is called "wing stridulation". This raises the question, whether these movements could be produced by the stridulation and/or flight pattern generator. To answer this, intracellular recordings have been made from interneurons of both pattern generators (Shütze, 1999). Unfortunatelly no interneurons in the thoracic ganglion have been found, which control both wing and leg stridulation. Schütze proposed that the brain might be involved in this alternation of sound generating appendages in St. rubicundus (Shütze, 1999).

The wing flapping is one of the courtship elements performed by the females, responding to male song in Ch.b. Its performance indicates conspecific song recognition (Fig. 3-4). The role of this behavioral act stimulated pharmacologically in males Ch.b. following leg stridulation (section 3.6.4) is difficult to predict. It could be a courtship element induced by sex-specific song recognition, analogous to those performed by the female. On the other hand, the wing movements, induced pharmacologically in male Ch.b. might be close related to the jumping, done by the males in the phonotactical mate approach. In one observation on freely moving animals, a male in response to a female stridulation after short singing, jumped vigorously towards the female and performed the next courtship song sequence. During the jumps actually he did wing movements (Fig. 3-3).

4.14 The brain may exist components of the stridulatory patterngenerating network

Since almost no electrophysiological data is available from the brain of Ch.b. and other species with complex singing patterns nothing is known about the role of the brain in the production of the rapid stridulatory movements. The 
induction of wing flapping together with leg stridulatory movements suggests a possible role of bifunctional neurons in the control of both fast wing and leg movements. In addition, the work from Schütze (1999) on another species with rapid stridulatory movements Stenobothrus rubicundus has failed to demonstrate such neurons in the thorax. He has suggested that such bifunctional role might play the brain. Moreover single stridulatory up-and downmovements ("single chirps" Fig. 3-25, Fig. 3-70) not structured into speciesspecific sequences of $1^{\text {st }}$ order open the hypothesis that the brain of Ch.b. can also be involved into generation of the rapid stridulatory movements, most likely through flight descending neurons, whose activtity can be superimposed on the neurons triggering slower stridulatory movements similar to those seen in O.V. The only brain interneuron, which has triggerd stridulation is local and the stridulatory movements triggered are not specific (Hedwig, 1995). Additional electrophysiological data are needed to reveal the role of the brain in rapid stridulatory movements.

On the other hand, in Ch.b. the singing pattern includes song sequences $1^{\text {st }}$ order preceding and following by pauses. Thus the level of excitation oscillates as it is shown in Fig. 4-6. A single stimulus of proctolin or muscarine can elicit several song sequences, which is a feature of one intrinsic oscillator (CPG). In contrast to O.v., in Ch.b. with the accumulation of excitation the durations of the song sequences ( $1^{\text {st }}$ order) and the pauses in between decrease proportional that indicates a step modulation (pacemaking) of the oscillations.

A models explaining the performance of entire song (sequence $2^{\text {nd }}$ order) is presented in Fig. 4-6 and mechanism for alternation of the singing parameters by co-transmitters is shown in Fig. 4-7. 


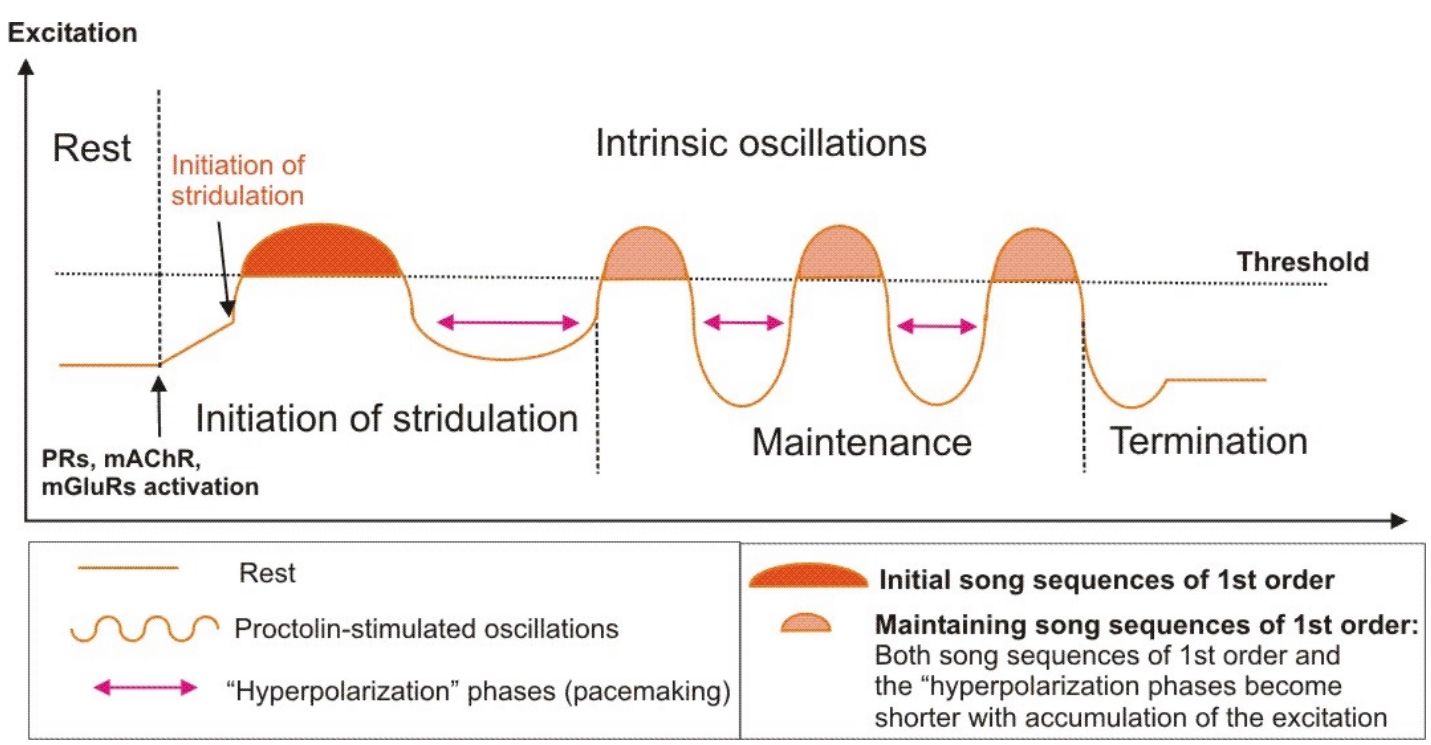

Fig. 4-6 Hypothetical model of proctolin-stimulated stridulatory activity in Ch.b. Entire song (sequence $2^{\text {nd }}$ order) is triggered by PRs activation. Intrinsic brain oscillator once activated performed the sequences $1^{\text {st }}$ order without additional inputs.

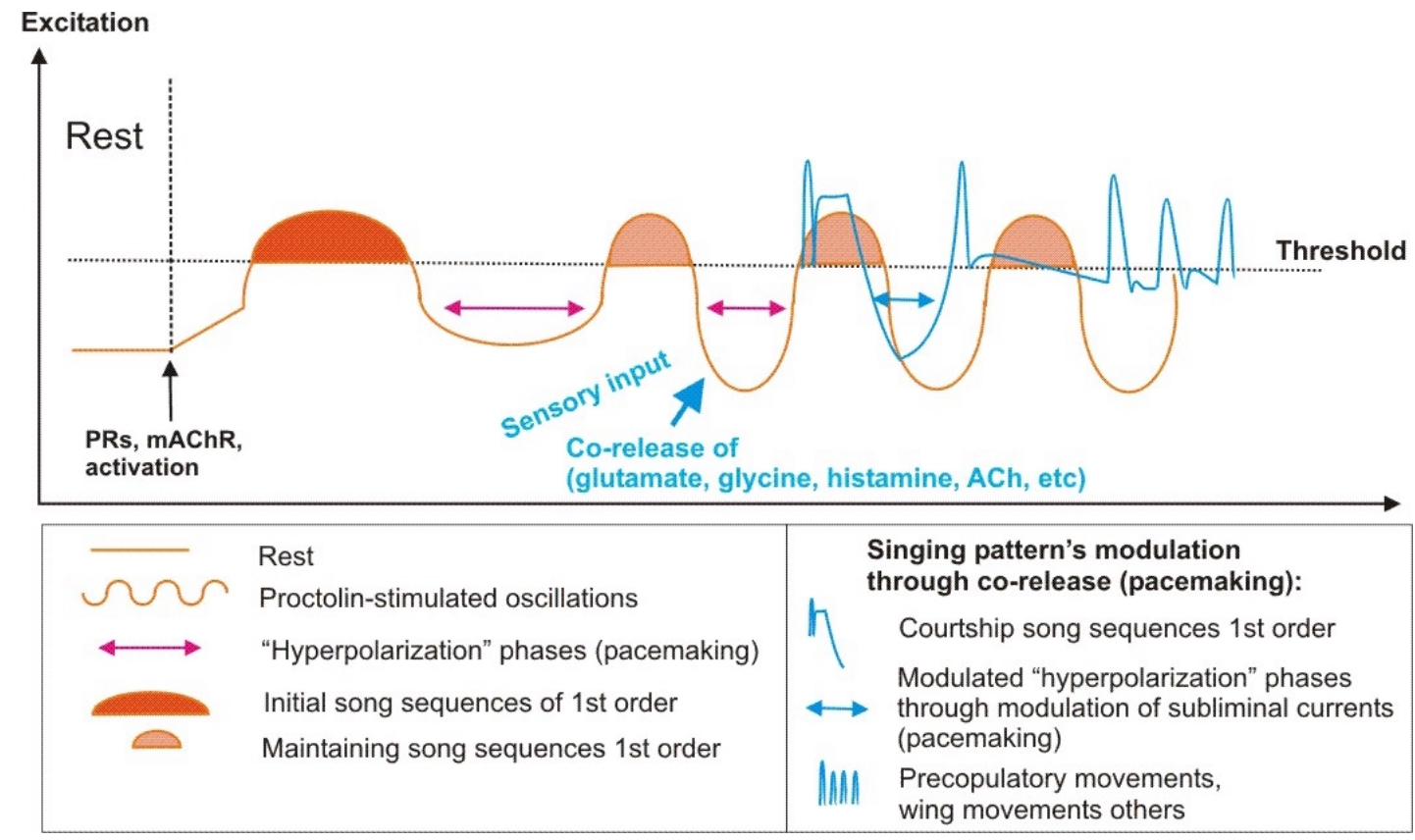

Fig. 4-7 Hypothetical role of co-released transmitters in "switch"-mechanisms between calling and courtship song, courtship and other movements during the mate approach in male Ch.b. Additional excitatory signal from sensory systems (auditory brain neuropile) may induce co-release of other transmitters within the control circuit, which can alter the oscillations, stimulated by proctolin. Modulation of subliminal currents, involved in maintaining the oscillation can allter the firing pattern of the control circuit (pacemaking). 


\subsection{Differences between the cephalic stridulatory control systems of}

\section{O.v. and Ch.b.}

In contrast to O.V., the cephalic control of singing has not been intensively studied with electrophysiological methods in Ch.b. Moreover, no data about the brain control of sound

production

grasshoppers

complex

singing

patterns and rapid

stridulatory

movements

(Ch.b.,

\section{Stenobothrus}

rubicundus etc.) has

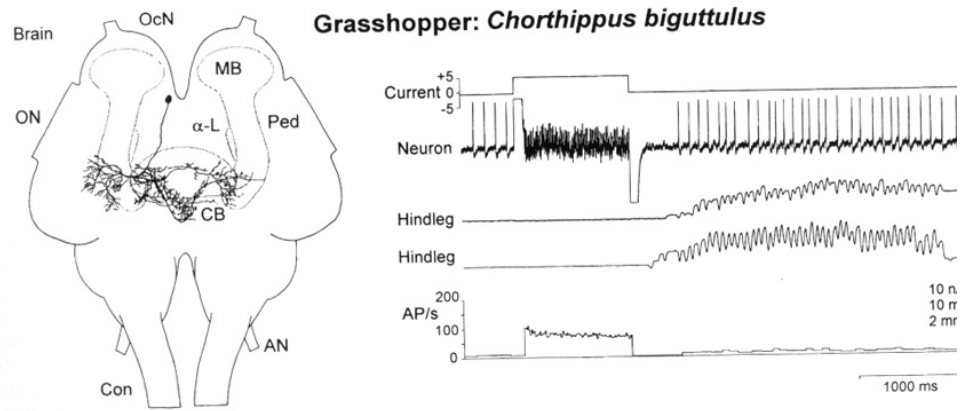

Fig. 4-8 Local interneuron in the brain of Ch.b. that control the stridulation (from Hedwig, 1995)

been collected to date. However, the only local brain neuron, other than the descending command neurons, that had a strong impact on the initiation of stridulation was identified in Ch.b. (Hedwig, 1995). Based on its anatomy (arborization in lateral protocerebrum) and stimulation effect (stridulation starts more than $1 \mathrm{sec}$ after the beginning of high frequency activation and the neuron's activity is not required during stridulation; see Fig. 4-8) this neuron may connect the neural filter for recognition of sex-specific singing with the control circuits that initiate approach and singing in the male. The descending command neurons have not been electrophysiologically characterized and identified histo-chemically in Ch.b.

The present pharmacological study collected indications that may suggest differences between the cephalic stridulatory control systems of O.v. and Ch.b., which could be regarded as representatives of species with slow and fast stridulatory movements:

- nAChRs-activation seems to be not sufficient to initiate and maintain singing in Ch.b. (section 3.4.6), which is easily accomplished in O.V.

- Dopamine successfully triggers stridulation in O.v. but not in Ch.b.

- Glutamate could not elicit any leg movement in O.v. (Ocker et al., 1995), while in Ch.b. triggers precopulatory movements and may 
play a role in switch mechanisms between different movement patterns

- Wing flapping as an element of the courtship of species with fast stridulatory movements occurred in Ch.b. but not in O.v.

- With the accumulation of the excitation of the stridulatory activity the duration of songs sequences decreased in Ch.b. in contrast to O.v. (oscillatory stridulatory activity vs. gradual arousal of excitation)

It will be important to identify and characterize electrophysiologically (combined with pharmacological stimulations) the brain neurons controlling the singing Ch.b. (or Stenobothrus rubicundus), in order to compare the neuronal mechanisms underlying both stridulatory pattenrs (slow and rapid stridulatory movements). 


\section{Summary}

The present thesis investigated the contribution of the neuropeptide proctolin to the cephalic control of sound production and associated behaviors in the gomphocerine grasshopper Chorthippus biguttulus (Ch.b). For this purpose, small amounts of proctolin, other transmitters and modulators, and drugs known to interfere with specific physiological mechanisms were pressure injected into the protocerebrum of immobilized but otherwise intact grasshoppers still capable of generating their species-specific sound signals. The major results of these studies are as follows:

- Injections of proctolin into the protocerebrum can elicit species-specific stridulation in both male and female Ch.b. The stimulated behavior is similar to the natural stridulation with respect to both the temporal structure (syllables, chirps, song sequences of $1^{\text {st }}$ and $2^{\text {nd }}$ order) and patterns of stridulatory movements of the hindlegs (e.g. chirps usually consisting of 6 syllables, two different patterns performed by the hindlegs that change their role after every few sequences of $1^{\text {st }}$ order).

- $\quad$ Proctolin can stimulate both calling and courtship song, the latter being characterized by shorter sequences of $1^{\text {st }}$ order. Since proctolin, when injected to the same site within the brain as muscarine, elicited both song sequences of shorter duration and additional courtship elements (wing flapping, precopulatory movements), its synaptic release may preferentially be associated with courtship and could probably trigger switches between calling and courtship singing when a con-specific female shows copulatory readiness.

- Compared with the stimulation of stridulation by muscarine, proctolin generally elicited shorter stridulation after a shorter latency. Effective stimulation sites for proctolin seemed to be restricted to the anterior portion of the central body complex, protocerebral bridge and the dorsal neuropil to the central complex, while muscarine could also stimulate singing in more posterior regions of the protocerebrum.

- mAChRs-activation may lead to prolonged calling singing, depending on abiotic factors (temperature, light etc.). In addition mAChRs-activation is not necessary for proctolin-stimulated stridulation. nAChRs-activation may play 
secondary role in the cephalic control of singing in Ch.b. Modulation of AChE activity was found to affect both muscarine and proctolin-stimulated responses.

- The stridulatory activity should be combined and coordinate with another complex behavioral programs (sex-specific song recognition, orientation and phonotaxis). The switch between them in a step-wise manner, without a decrease of the basal excitation to sing is a main feature of the courtship in Ch.b. Proctolin may play a role in the control of background excitation that allows an alternation between the courtship song and the phonotactical mate approach.

- Stimulatory effects of proctolin are terminated by desensitization of proctolin receptors, that appeared with short inter-stimulus intervals.

- Proctolin mediates its excitatory effects in the neural circuits that control stridulation through receptors that activate a phospholipase C-triggered second messenger pathway. This was demonstrated by several experiments, in which drugs and ions known to interfere with the phospholipase C pathway altered the duration of proctolin-stimulated stridulation. These studies revealed that phospholipase $\mathrm{C}$, protein kinase $\mathrm{C}$ and calcium released from intracellular stores mediate the proctolin receptor-initiated excitation. Receptor specific activation of PLC signaling pathway by muscarine (mAChRs), proctolin (PRs), glutamate (mGluRs $(1,5)$ ) within the control circuit is postulated. The activation of the adenylate cyclase affected only partly proctolin-stimulated singing.

- Another stimulation protocol was suggested, that solves some of the problems of the present pharmacological approach and allows to investigate processes of co-release (transmitters, modulators and local hormones) within the control circuit.

- A number of other signaling molecules were tested for a potential contribution to the cephalic control of stridulation in Ch. biguttulus. Stridulation could also be stimulated by glutamate (via mGluRs, coupled to PLC pathway), while dopamine seemed to suppress proctolin-stimulated singing. Co-stimulation of proctolin with a second transmitter could alter the time course of proctolin-stimulated stridulation (glycine, histamine) or lead to 
the appearance of other courtship elements (glutamate). Although interpretation of these results is difficult due to the lack of knowledge about individual neurons within the control circuits, possible mechanisms and functions of the co-transmission in the controlling neuronal circuit are discussed.

- The present pharmacological study collected indications that may suggest differences between the cephalic stridulatory control systems of O.v. and Ch.b., which could be regarded as representatives of species with slow and fast stridulatory movements:

- nAChRs-activation plays a main role in sound production in O.v., in contrast to Ch.b.

- Dopamine successfully triggers stridulation in O.v. but not in Ch.b.;

- Glutamate could not elicit any leg movement in O.v., while in Ch.b. triggers precopulatory movements and may play a role in switch mechanisms between different movement patterns;

- Wing flapping as an element of the courtship of species with fast stridulatory movements occurred in Ch.b. but not in O.v.

- With the accumulation of the excitation of the stridulatory activity the duration of songs sequences of $1^{\text {st }}$ order decreased in Ch.b. in contrast to O.v. (oscillatory activity vs. gradual arousal of excitation). 


\section{Bibliography}

Acevedo, L.D., Hall, W.M., and Mulloney, B. (1994) Proctolin and excitation of the crayfish swimmeret system. Journal of Comparative Neurology 345, 612-627.

Adams, M.E. and O'Shea, M. (1983) Peptide cotransmitter at a neuromuscular-junction. Science 221, 286-289.

Amat, C., Sinakevitch-Pean, I., and Hue, B. (1997) Proctolin-like immunoreactivity in dorsal paired median neurons generating plateau action potentials in cockroach Periplaneta americana. Neuroscience Research Communications 29, 263-268.

Anderson, M.S., Halpern, M.E., and Keshishian, H. (1988) Identification of the neuropeptide transmitter proctolin in Drosophila larvae: Characterization of muscle fiber-specific neuromuscular endings. Journal of Neuroscience 8, 242-255.

Andries, J. C., Belemtougri G., et al., (1991). Multiple Peptide Immunoreactivities in the Nervous-System of Aeschna-Cyanea (Insecta, Odonata) - an Immunohistochemical Study Using Antisera to Cholecystokinin Octapeptide, Somatoliberin, Vasoactive-Intestinal-Peptide, Motilin and Proctolin. Histochemistry 96 (2): 139-148.

Aubele, E. and Klemm, N. (1977) Origin, destination and mapping of tritocerebral neurons in the locust. Cell and Tissue Research 178, 199-219.

Baines, R. A., Lange, A. B., Downer, R. G. (1990) Proctolin in the innervation of the locust mandibular closer muscle modulates contractions through the elevation of inositol trisphosphate. Journal of Comparative Neurology 297(4), 479-86.

Baines, R. A. and R. G. H. Downer (1991). The Role of Proctolin in Maintaining Contractions of the Locust (Locusta-Migratoria) Mandibular Closer Muscle. Journal of Insect Physiology 37(6): 431-439.

Baines, R. A. and Downer, R. G. H. (1992) Comparative-Studies on the Mode of Action of Proctolin and Phorbol-12,13-Dibutyrate in Their Ability to Contract the Locust Mandibular Closer Muscle. Archives of Insect Biochemistry and Physiology 20(3), 215-229.

Baines, R.A., Walther, C., Hinton, J.M., Osborne, R.H., and Konopinska, D. (1996) Selective activity of a proctolin analogue reveals the existence of two receptor subtypes. Journal of Neurophysiology 75, 2647-2650. 
Banner, S.E., Cattell, K.J., and Osborne, R.H. (1986) Effects of Agonists on 5-HT and Octopamine receptors in the Foregut of Schistocerca gregaria. British Journal of Pharmacology 89, 825P (Abstract)

Banner, S.E., Osborne, R.H., and Cattell, K.J. (1987) The pharmacology of the isolated foregut of the locust Schistocerca gregaria - 1. The effect of a range of putative neurotransmitters. Comparative Biochemistry And Physiology C 88, 131-138.

Banner, S.E., Osborne, R.H., Walker, M.J., and Cattell, K.J. (1989) The pharmacology of the isolated foregut of the locust Schistocerca gregaria - III. Identification of an octopamine $_{2}$ receptor. Comparative Biochemistry And Physiology C 92, 333-336.

Banner, S.E., Seale, L., Osborne, R.H., and Cattell, K.J. (1988) Effects of proctolin on fore and hind gut of the locust Schistocerca gregaria. Pesticide Science 23, 355-357.

Banner, S.E., Wood, S.J., Osborne, R.H., and Cattell, K.J. (1990) Tyramine antagonizes proctolin-induced contraction of the isolated foregut of the locust Schistocerca gregaria by an interaction with octopamine2 receptors. Comparative Biochemistry and Physiology - C Pharmacology Toxicology and Endocrinology 95, 233-236.

Bartos, M., Allgauer, C., Eckert, M., and Honegger, H.W. (1994) The antennal motor system of crickets: Proctolin in slow and fast motoneurons as revealed by double labelling. European Journal of Neuroscience 6, 825-836.

Bartos, M. and Honegger, H.W. (1997) Impact of motor activity and antennal mechanosensory input on the intensity of proctolin-like immunoreactivity in antennal motoneurons of crickets (Gryllus bimaculatus). Journal Of Comparative Physiology A-Sensory Neural And Behavioral Physiology 181, 59-70.

BartoszBechowski, H., Rosinski, G., Konopinska, D., Sujak, P., and Sobotka, W. (1990) Further studies on proctolin analogues modified in position 2 of the peptide chain and their influence on heart-beat frequency of insects. International Journal of Peptide and Protein Research 36, 450-456.

Bauer, C.K. (1991) Modulatory action of proctolin in the locust (Locusta migratoria) antennal motor system. Journal Of Insect Physiology 37, 663-673.

Becherer, C. and Schmid, A. (1999) Distribution of g-aminobutyric acid-, proctolin-, Peripaneta hypertrehalosaemic hormone-, and FMRFamide-like immunoreactivity in the visual ganglia of the spider Cupiennius salei Keys. Comparative Biochemistry and Physiology - A Physiology 122, 267-275. 
Belanger, J.H. and Orchard, I. (1993) The locust ovipositor opener muscle - properties of the neuromuscular system. Journal of Experimental Biology 174, 321-342.

Beltz, B.S., Pontes, M., Helluy, S.M., and Kravitz, E.A. (1990) Patterns of appearance of serotonin and proctolin immunoreactivities in the developing nervous system of the American lobster. Journal of Neurobiology 21, 521-542.

Berridge, M.J. (1993) Inositol triphosphate and calcium signaling. Nature 361, 315-325.

Bernstein, H.G., Eckert, M., Penzlin, H., and Dorn, A. (1984) Proctolin-related material in the mouse brain as revealed by immunohistochemistry. Neuroscience Letters $\mathbf{4 5}$, 229-232.

Bernstein, H.G., Eckert, M., Penzlin, H., Vieweg, U., ROSE, I., and Dorn, A. (1986) Proctolin immunoreactive neurons in the human brain stem. Acta Histochemica 80, 111-114.

Bicker, G. and Menzel, R., (1989) Chemical codes of the control of behaviour in arthropods. Nature 337, 33-39.

Bishop, C.A. and O'Shea, M. (1982) Neuropeptide proctolin (H-Arg-Try-Leu-Pro-Thr-OH): Immunocytochemical mapping of neurons in the central nervous system of the cockroach. Journal of Comparative Neurology 207, 223-238.

Bishop, C.A., O'Shea, M., and Miller, R.J. (1981) Neuropeptide proctolin (H-Arg-Tyr-LeuPro-Thr-OH): Immunological detection and neuronal localization in insect central nervous system. Proceedings Of The National Academy Of Sciences Of The United States Of America 78, 5899-5902.

Bishop, C. A., M. E. Krouse, et al. (1991). Peptide Cotransmitter Potentiates CalciumChannel Activity in Crayfish Skeletal-Muscle. Journal of Neuroscience 11(1): 269276.

Blitz, D.M., Nusbaum, M.P. (1997) Motor pattern selection via inhibition of parallel pathways. The Journal of Neuroscience 17(13), 4965-75.

Blitz, D.M., Christie, A.E., Coleman, M.J., Norris, B.J., Marder, E. and Nusbaum, M.P. (1999) Different proctolin neurons elicit distinct motor patterns from a multifunctional neuronal network. The Journal of Neuroscience 19(13), 5449-63.

Blitz, D.M. and Nusbaum, M.P. (1999) Distinct functions for cotransmitters mediating motor pattern selection. The Journal of Neuroscience 19(16), 6774-83. 
Bräunig, P. (1990) The morphology of suboesophageal ganglion cells innervating the nervus corporis cardiaci II of the locust. Cell and Tissue Research 260, 95-108.

Breer, H. (1981) Characterisation of synaptosomes from the central nervous system of insects. Neurobiochemistry International 3, 155-163.

Breer, H. (1991) Molecular mechanisms of olfactory signal transduction. Journal Of General Physiology 98, A4-A4

Breer, H. and Jeserich, G. (1980) A microscale floatation technique for the isolation of synaptosomes from nervous tissue of Locusta migratoria. Insect Biochemistry 10, 457-463.

Breer, H. and Knipper, M. (1984) Characterisation of Acetylcholine release from insect synaptosomes. Insect Biochemistry 14, 337-344.

Breer, H. and Sattelle D.B. (1987) Molecular properties and functions of insect acelylcholine receptors. Journal of Insect Physiology 33, 771-790.

Brown, B.E. (1965) Pharmacologically active constituents of the cockroach corpus cardiacum: Resolution and some characteristics. General And Comparative Endocrinology 5, 387-401.

Brown, B.E. (1967) Neuromuscular Transmitter Substance in insect visceral muscle. Science 155, 595-597.

Brown, B.E. (1975) Proctolin: a peptide transmitter candidate in insects. Life Sciences 17, $1241-1252$.

Brown, B.E. (1977) Occurance of proctolin in six orders of insects. Journal Of Insect Physiology 23, 861-864.

Brown, B.E. and Starratt, A.N. (1975) Isolation of Proctolin, a myotropic peptide, from Periplaneta americana. Journal Of Insect Physiology 21, 1879-1881.

Brustle, B., Kreissl, S., Mykles, D.L., Rathmayer, W. (2001) The neuropeptide proctolin induces phosphorylation of a $30 \mathrm{kDa}$ protein associated with the thin filament in crustacean muscle. The Journal of Experimental Biology 204, 2627-2635.

Burnstock, G. (1976) Do some cells release more than one transmitter? Neurosciences 1, 239-248.

Burrows, M. (1996) The neuroanatomy of an insect brain,

Cantera, R. and Nässel, D.R. (1991) Dual peptidergic innervation of the blowfly hindgut: A light- and electron microscopic study of FMRFamide and proctolin immunoreactive 
fibers. Comparative Biochemistry and Physiology - C Pharmacology Toxicology and Endocrinology 99, 517-525.

Chapman, R.F. (1969) The Insects - structure and function, 1 Ed., London, The English Universities Press.

Chapman, R.F. (1985) Structure of the Digestive System. In: Comprehensive insect Biochemistry, Physiology and Pharmacology., 165-211. Edited by Kerkut, G.A. and Gilbert, L.I., London, Permagon Press.

Christie, A.E., Skiebe, P. and Marder, E. (1995) Matrix of neuromodulators in neurosecretory structures of the crab Cancer borealis. The Journal of Experimental Biology 198, 2431-2439.

Chyb, S., Hevers, W., Forte, M., Wolfgang, W.J., Selinger, Z. and Hardie R.C. (1999) Modulation of the light response by cAMP in Drosophila photoreceptors. The Journal of Neuroscience 19(20), 8799-8807.

Clarke, K.U. and Grenville, H. (1960) Nervous control of movements in the foregut of Schistocerca gregaria Forsk. Nature 186, 98-99.

Coleman, M.J., Nusbaum, M.P., Cournil, I. and Claiborne, B.J. (1992) Distribution of modulatory inputs to the stomatogastric ganglion of the crab, Cancer borealis. The Journal of comperative neurology 325, 581-594.

Coleman, M.J., Konstant, P.H., Rothman, B.S., and Nusbaum, M.P. (1994) Neuropeptide degradation produces functional inactivation in the crustacean nervous system. Journal of Neuroscience 14, 6205-6216.

Cook, B.J. and Holman, G.M. (1979) The action of proctolin and L-glutamic acid on the visceral muscles of the hindgut of the cockroach Leucophaea maderae. Comparative Biochemistry And Physiology C 64C, 21-28.

Cook, B.J. and Holman, G.M. (1985) The role of proctolin and glutamate in the excitationcontraction coupling of insect visceral muscle. Comparative Biochemistry And Physiology C 80, 65-73.

Davis, N.T., Homberg, U., Teal, P.E.A., Altstein, M., Agricola, H.J., and Hildebrand, J.G. (1996) Neuroanatomy and immunocytochemistry of the median neuroendocrine cells of the subesophageal ganglion of the tobacco hawkmoth, Manduca sexta: Immunoreactivities to PBAN and other neuropeptides. Microscopy Research And Technique 35, 201-229. 
Davis, N.T., Velleman, S.G., Kingan, T.G., and Keshishian, H. (1989) Identification and distribution of a proctolin-like neuropeptide in the nervous system of the gypsy moth, Lymantria dispar, and in other Lepidoptera. Journal of Comparative Neurology 283, 71-85.

Dircksen, H. and Homberg, U. (1995) Crustacean cardioactive peptide-immunoreactive neurons innervating brain neuropils, retrocerebral complex and stomatogastric nervous system of the locust Locusta migratoria. Cell and Tissue Research 279, 495-515.

Dunbar, S.J. and Piek, T. (1983) The action of iontophoretically applied L-glutamate on an insect visceral muscle. Archives Of Insect Biochemistry And Physiology 1, 93-103.

Duve, H., WREN, P., and Thorpe, A. (1995) Innervation of the foregut of the cockroach Leucophaea -maderae and inhibition of spontaneous contractile activity by callatostatin neuropeptides. Physiological Entomology 20, 33-44.

Eckert, M., Agricola, H., and Penzlin, H. (1981) Immunocytochemical identification of proctolin-like immunoreactivity in the terminal ganglion and hindgut of the cockroach Periplaneta americana. Cell and Tissue Research 217, 633-645.

El Manira, A., RossiDurand, C., and Clarac, F. (1991) Serotonin and proctolin modulate the response of a stretch receptor in crayfish. Brain Research 541, 157-162.

Elsner, N. (1974) Neuroethology of sound production in gomphocerine grasshoppers (Orthoptera: Acrididae) I. Song patterns and stridulatory movements. Journal of comparative Physiology 88, 67-102

Elsner, N. (1995) The search for the neural centers of cricket and grasshopper song. In: Schildberger K, Elsner N (Eds.): Neural Basis of Behavioural Adaptations. Gustav Fischer, Stuttgart, Jena: 167-194.

Ernst, K.D., Boeckh, J., and Boeckh, V. (1977) A neuroanatomical study on the organisation of the central antennal pathways in insects. II. Deutocerebral connections in Locusta migratoria and Periplaneta americana. Cell and Tissue Research 176, 285-308.

Facciponte, G., Miksys, S., and Lange, A.B. (1996) The association of proctolin with a ventral abdominal muscle of Locusta migratoria. Journal Of Comparative Physiology A-Sensory Neural And Behavioral Physiology 178, 269-278. 
Felder, C. (1995) Muscarinic acetylcholine receptors: signal transduction through multiple effectors. The FASEB Journal 9, 619-625.

Fitch, J. and Djamgoz, M.B.A. (1988) Proctolin potentiates synaptic transmission in the central nervous system of an insect, Comp. Biochem. Physiol. 89C:109-112.

Ganfornina, M.D., Sánchez, D., and Bastiani, M.J. (1996) Embryonic developement of the enteric nervous system of the grasshopper Schistocerca gregaria. The Journal of Comparative Neurology 372, 581-596.

Golowasch, J., F. Buchholtz, et al. (1992) Contribution of Individual Ionic Currents to Activity of a Model Stomatogastric Ganglion Neuron. Journal of Neurophysiology 67(2), 341-349.

Golowasch, J. and Marder E. (1992) Proctolin Activates an Inward Current Whose Voltage Dependence Is Modified by Extracellular Ca-2+. Journal of Neuroscience 12(3), 810-817.

Gray, A.S., Hancock, J.T., and Osborne, R.H. (2000) Proctolin antagonists bind to [ $\left.{ }^{3} \mathrm{H}\right]-$ proctolin binding sites inthe locust hindgut. Peptides 21(2):189-96

Gray, A.S. and Osborne, R.H. (1994) [alpha-methyl-tyrosine ${ }^{2}$ ]-proctolin blocks proctolin receptors in the locust foregut. British Journal of Pharmacology 112, U 66-U 66

Gray, A.S., Osborne, R.H., and Jewess, P.J. (1994) Pharmacology of proctolin receptors in the isolated foregut of the locust Schistocerca gregaria - identification of [alphamethyl-L- tyrosine ${ }^{2}$ ]-proctolin as a potent receptor antagonist. Journal Of Insect Physiology 40, 595-600.

Gray, E.G. and Whittaker, V.P. (1962) The isolation of nerve endings from brain: an electron microscopic study of cell fragments derived by homogenisation and centrifugation. Journal of Anatomy 96, 79-87.

Groome, J.R., Tillinghast, E.K., Townley, M.A., Vetrovs, A., Watson WH, I.I.I., Hunt, D.F., Griffin, P.R., Alexander, J.E., and Shabanowitz, J. (1990) Identification of proctolin in the central nervous system of the horseshoe crab, Limulus polyphemus.

Peptides 11, 205-211.

Groome, J.R., Townley, M.A., Detschaschell, M., and Tillinghast, E.K. (1991) Detection and isolation of proctolin-like immunoreactivity in arachnids - possible cardioreulatory role for proctolin in the orb-weaving spiders Argiope and Araneus. Journal Of Insect Physiology 37, 9-19. 
Gundel, M. and Penzlin, H. (1978) The neuronal connections of the frontal ganglion of the cockroach Periplaneta americana . Cell and Tissue Research 193, 353-371.

Hammond, C. (2001) Cellular and Molecular Neurobiology. Academic Press, A Harcourt Science and Technology Company.

Hedwig, B. (1986a) On the role in stridulation of plurisegmental interneurons of the acridid grasshopper Omocestus viridulus L. I. Anatomy and physiology of descending cephalothoracic interneurons. Journal of Comparative Physiology A 158, 413-427.

Hedwig, B. (1994) A cephalo-thoracic command system controls stridulation in the acridid grasshopper Omocestus viridulus L. J Neurophysiol 72, 2015-2025.

Hedwig, B. and Heinrich, R. (1997) Identified descending brain neurons control different stridulatory motor patterns in an acridid grasshopper. Journal of Comparative Physiology 180, 285-294.

Hedwig, B. (2000) A highly sensitive opto-electronic system for the measurement of movements. Journal of Neuroscience Methods 100, 165-171.

Hedwig, B. (2000) Control of cricket stridulation by a command neuron: efficacy depends on the behavioral state. Journal of Neurophysiology 83, 712-722.

Hedwig, B. (2001) Singing and hearing: neuronal mechanisms of acoustic communication in Orthopterans. Zoology 103, 140-149.

Heinrich, R. (1995) Mikrochirurgische und pharmakologische Untersuchungen über die neuronalen Grundlagen des Heuschreckengesangs. Dissertation zur Erlangung des Doktorgrades der Mathematisch-Naturwissenschaftlichen Fachbereiche der Georg-August-Universität zu Göttingen.

Heinrich, R. and Elsner, N. (1997) Central nervous control of hindleg coordination in stridulating grasshoppers. Journal of comparative Physiology 180, 257-269.

Heinrich, R., Hedwig, B., and Elsner, N. (1997) Cholinergic activation of stridulatory behaviour in the grasshopper Omocestus viridulus (L.). Journal of Experimental Biology 200, 1327-1337.

Heinrich, R., Jacobs, K. and Lakes-Harlan, R. (1998) Tracing of a neuronal network in the locust by pressure injection of markers into a synaptic neuropil. Journal of Neuroscience Methods 80, 81-89. 
Heinrich, R., Rozwod, K., and Elsner, N. (1998) Neuropharmacological evidence for inhibitory cephalic control mechanisms of stridulatory behaviour in grass-hoppers. Journal of Comparative Physiology [A] 183, 389-399.

Heinrich, R., Cromarty, S., Hörner, M., Edwards, D.H. and Kravitz, E.A. (1999) Autoinhibition of serotonin cells: An intrinsic regulatory mechanism sensitive to the pattern of usage of the cells. Proceedings Of The National Academy Of Sciences Of The United States Of America 96, 2473-2478.

Heinrich, R., Braunig, P., Walter, I., Schneider, H., Kravitz E.A. (2000) Aminergic neuron systems of lobsters: morphology and electrophysiology of octopamine-containing neurosecretory cells. Journal of comparative physiology 186 (7-8), 617-629.

Heinrich, R., Wenzel, B., and Elsner, N. (2001a) Pharmacological brain stimulation releases elaborate stridulatory behaviour in gomphocerine grasshoppers conclusions for the organization of the central nervous control. Journal of Comparative Physiology [A] 187, 155-169.

Heinrich, R., Wenzel, B., and Elsner, N. (2001b) A role for muscarinic excitation: control of specific singing behavior by activation of the adenylate cyclase pathway in the brain of grasshoppers. Proc Nat Acad Sci USA 98, 9919-9923.

Heinrich, R. (2002) Impact of descending brain neurons on the control of stridulation, walking and flight in Orthoptera. Microscopy research and technique 56, 292-301.

Helle, J., Dircksen, H., Eckert, M., Nassel, D.R., Sporhaseeichmann, U., and Schurmann, F.W. (1995) Putative neurohemal areas in the peripheral nervous-system of an insect, Gryllus-bimaculatus, revealed by immunocytochemistry. Cell and Tissue Research 281, 43-61.

von Helversen, O. and Elsner, N. (1977) The stridulatory movements of acridid grasshoppers recorded with an opto-electronic device. Journal of Comparative Physiology 122, 53-64.

von Helversen, O. (1997) Acoustic communication and orientation in grasshoppers. In: Orientation and Communication in Arthropods, ed. by M. Lehrer; Birkhäuser Verlag Basel/Switzerland; pp. 301-341

Hertel, W. and H. Penzlin (1992). Function and Modulation of the Antennal Heart of Periplaneta- Americana (L). Acta Biologica Hungarica 43 (1-4): 113-125. 
Hignam, K.C., Hill, L., and Mordue, W. (1966) The endocrine system and oocyte growth in Schistocerca in relation to starvation and frontal ganglionectomy. Journal Of Insect Physiology 12, 977-994.

Hinton, J.M. and Osborne, R.H. (1995) Proctolin receptor in the foregut of the locust Schistocerca gregaria is linked to inositol phosphate 2nd-messenger system. Journal Of Insect Physiology 41, 1027-1033.

Hinton, J.M. and Osborne, R.H. (1996) Effects of proctolin and related analogs on inositol phosphate production in the foregut of the locust Schistocerca gregaria. Insect Biochemistry And Molecular Biology 26, 111-117.

Hinton, J.M., Osborne, R.H., BartoszBechowski, H., and Konopinska, D. (1996) Myotropic effects of proctolin analogues, modified in position 2 of the peptide chain, on the foregut of the locust Schistocerca gregaria. Journal Of Insect Physiology 42, 449454.

Hinton, J.M., Osborne, R.H., Odell, B., Hammond, S.J., and Blagbrough, I.S. (1995) Cycloproctolin and [alpha-methyl-L-tyr]-proctolin are potent antagonists of proctolin-induced inositol phosphate production in locust foregut homogenates. Bioorganic and Medicinal Chemistry Letters 5, 3007-3010.

Hinton, J.M., Nejad, M., Issberner, J.P., Hancock, J.T., Osborne, R.H. (1998) Muscarinic acetylcholine and proctolin receptors in the foregut of the locust Schistocerca gregaria: role of inositol phosphates, protein kinase $C$ and calcium in second messenger effects. Insect Biochemistry and Molecular Biology 28: 331-343.

Hiripi, L., Rosza, K.S. and Miller, T.A. (1979). Effect of proctolin on the adenylate and guanilate cyclase in the Locust brain at various developmental stages. Experientia $35,1287-1288$.

Holets, V.R., Hokfelt, T., and Ude, J. (1987) A comparative study of the immunohistochemical localization of a presumptive proctolin-like peptide, thyrotropin-releasing hormone and 5-hydroxytryptamine in the rat central nervous system. Brain Research 408, 141-153.

Holman, G.M. and Cook, B.J. (1972) Isolation, partial purification and characterisation of a peptide which stimulates the hindgut of the cockroach Leucophaea maderae (Fabr.). Biological Bullitin of Woods Hole 142, 446-460. 
Holman, G.M. and Cook, B.J. (1979b) Evidence for proctolin and a second myotropic peptide in the cockroach, Leucophaea maderae, determined by bioassay and HPLC analysis. Insect Biochemistry 9, 149-154.

Holman, G.M. and Cook, B.J. (1979a) The analytical determination of proctolin by HPLC and its pharmacological action in the stable fly. Comparative Biochemistry And Physiology C 62, 231-235.

Holman, G.M., Cook, B.J., and Wagner, R.M. (1984) Isolation and partial characterization of 5 myotropic peptides present in head extracts of the cockroach leucophaeamaderae. Comparative Biochemistry And Physiology C 77, 1-5.

Homberg, U., Vitzthum, H., Muller, M., and Binkle, U. (1999) Immunocytochemistry of GABA in the central complex of the locust Schistocerca gregaria: Identification of immunoreactive neurons and colocalization with neuropeptides. Journal of Comparative Neurology 409, 495-507.

Huber, F. (1974) Neural integration Central nervous system). In: The physiology of Insecta, 2 Ed., 4-90. Edited by Rockstein, M., London, Academic Press.

Isaac, R.E. (1986) Enzymatic degradation of proctolin in the CNS of the locust, Shistocerca gregaria, by membrane bound enzymes. In: Insect Neurochemistry and Neurophysiology, 207-210. Edited by Borkovec, A.B. and Gelman, D.B., New Jersey, The Human Press.

Isaac, R.E. (1987) Proctolin degradation by membrane peptidases from nervous tissues of the desert locust (Shistocerca gregaria). Biochemical Journal 245, 365-370.

Isaac, R.E. (1988) Neuropeptide-degrading endopeptidase activity of locust (Schistocerca gregaria) synaptic membranes. Biochemistry Journal 255, 843-847.

Isaac, R.E. (1988) Proctolin degradation by membrane peptidases from nerous tissues of the desert locust (Shistocerca gregaria). Biochemisty Journal 255, 843-847.

Isaac, R.E. (1997) Neuropeptide-degrading endopeptidase activity of locust (Shistocerca gregaria) synaptic membranes. Biochemistry Journal 255, 843-847.

Jedrusiak, J., Plech, A., Brus, R., Sobotka, W., and Konopinska, D. (1991) Studies on the central effects of [Lys $\left.{ }^{1}\right]$-proctolin, an analogue of proctolin. Bulletin Of The Polish Academy Of Sciences-Biological Sciences 39, 333-339. 
Jeziorski, M.C., Greenberg, R.M. and Anderson P.A.V. (2000) The molecular biology of ivertebrate voltage-gated Ca2+ channels. The Journal of Experimental Biology 203, 841-856.

Johnson, E.C., Garczynski, S.F., Park, D., Crim, J.W., Nässel, D.R. and Taghert, P.H. (2003) Identification and characterization of a $G$ protein-coupled receptor for the neuropeptide proctolin in Drosophila melanogaster. Proc Nat Acad Sci USA 100, 6198-6203.

Johnson, B. and Bowers, B. (1963) Axonal transport of neurosectretion from corpus cardiacum of Periplaneta. Science 141, 264-266.

Keshishian, H. and O'Shea, M. (1985) The acquisition and expression of a peptidergic phenotype in the grasshopper embryo. Journal of Neuroscience 5, 1005-1015.

Keshishian, H. and O'Shea, M. (1985) The distribution of a peptide neurotransmitter in the postembryonic grasshopper central nervous system. Journal of Neuroscience $\mathbf{5}$, 992-1004.

King, L.E., Sevala, V.M., and Loughton, B.G. (1995) The effect of substitutions at position3 on the binding and bioactivity of proctolin in locust hindgut and oviduct. Insect Biochemistry And Molecular Biology 25, 293-301.

Kingan, T.G. and Titmus, M. (1983) Radioenzymatic detection of proctolin in arthropods. Comparative Biochemistry And Physiology C 74, 75-78.

Knipper, M., MADEDDU, L., Breer, H., and MELDOLESI, J. (1986) BLACK-WIDOW SPIDER VENOM-INDUCED RELEASE OF NEUROTRANSMITTERS MAMMALIAN SYNAPTOSOMES ARE STIMULATED BY A UNIQUE VENOM COMPONENT (ALPHA-LATROTOXIN), INSECT SYNAPTOSOMES BY MULTIPLE COMPONENTS. Neuroscience 19, 55-\&

Konopinska, D. (1997) Insect neuropeptide proctolin and its analogues. An overview of the present literature. Journal of Peptide Research 49, 457-466.

Konopinska, D., BartoszBechowski, H., Rosinski, G., and Sobotka, W. (1993) New proctolin analogs modified in position-4 of the peptide- chain and their influence on the heart-beat frequency of insects. Bulletin Of The Polish Academy Of SciencesChemistry 41, 27-39.

Konopinska, D., Kazanowska, B., Sobotka, W., and Boguslawskajaworska, J. (1986b) An insect neuropeptide, proctolin - a new synthesis and its property of restoration of 
phagocytosis by defective human-pmn leukocytes. Bulletin Of The Polish Academy Of Sciences-Chemistry 34, 327-331.

Konopinska, D., Rosinski, G., BartoszBechowski, H., Lesicki, A., Sujak, P., and Sobotka, W. (1990) Role of guanidine group at the N-terminal proctolin chain in cardioexcitatory effects in insects. International Journal of Peptide and Protein Research 35, 12-16.

Konopinska, D., Rosinski, G., Lesicki, A., Sobotka, W., and Sujak, P. (1988b) [L-dopa²]proctolin - synthesis and its high cardioexcitatory effect on Periplaneta-americana L. Bulletin Of The Polish Academy Of Sciences-Chemistry 36, 17-20.

Konopinska, D., Rosinski, G., Lesicki, A., Sobotka, W., Sujak, P., and Kasprzyk, A. (1987) Synthesis and biological investigations of hyperglycemic peptide - periplanetin cc-2 from corpora cardiaca of cockroach Periplaneta americana L. Bulletin Of The Polish Academy Of Sciences-Chemistry 35, 125-131.

Konopinska, D., Rosinski, G., Lesicki, A., Sujak, P., Sobotka, W., and BartoszBechowski, H. (1988a) New N-terminal modified proctolin analogs - synthesis and their cardioexcitatory effect on insects. International Journal of Peptide and Protein Research 31, 463-467.

Konopinska, D., Rosinski, G., and Sobotka, W. (1992) New proctolin analogs modified in position 3 of peptide chain - Synthesis and their biological evaluation. Polish Journal of Pharmacology and Pharmacy 44, 505-514.

Konopinska, D., Rosinski, G., and Sobotka, W. (1992) Proctolin - Structure/biological function relationship studies. Insecticides: Mechanism of Action and Resistance 191-203.

Konopinska, D., Rosinski, G., Sobotka, W., and Plech, A. (1994) Proctolin and its analogues - structure/biological function relationship studies. Polish Journal Of Chemistry 68, 1437-1439.

Konopinska, D., Sobotka, W., Lesicki, A., Rosinski, G., and Sujak, P. (1986a) Synthesis of proctolin analogues and their cardioexcitatory effect on cockroach, Periplaneta americana L., and yellow mealworm, Tenebrio molitor L. International Journal of Peptide and Protein Research 27, 597-603.

Konopinska, D. and Rosinski, G. (1999) Proctolin, an insect neuropeptide. Journal of Peptide Science 5(12), 533-546. 
Kowalik-Jankowska, T., Janowska, E., Szeszel-Fedorowicz, W. and Konopinska D. (1975) Bonding of copper (II) ions by proctolin analogues modified in position 5 of the peptide chain. Journal of insect physiology 21, 1879.

Kuczer, M., Rosinski, G., Issberner, J., Osborne, R.H., and Konopinska, D. (1997) New proctolin analogues. Synthesis and myotropic effects in insects Tenebrio molitor and Schistocerca gregaria. In: Insects: Chemical, Physiological and Environmental aspects., 1 Ed., 172-175. Edited by Konopinska, D., Wroclaw, University of Wroclaw Press.

Kuczer, M., Rosinski, G., Issberner, J., Osborne, R.H., and Konopinska, D. (1998b) New proctolin analogues and their myotropic effects on heart of yellow mealworm Tenebrio molitor L. and foregut of locust Schistocerca gregaria L. Polish Journal of Pharmacology 50, 143-150.

Kuczer, M., Rosinski, G., Issberner, J., Osborne, R.H., and Konopinska, D. (1998a) New proctolin analogues: Synthesis and biological investigation in insects. Letters in Peptide Science 5, 387-389.

Kuczer, M., Rosinski, G., Issberner, J., Osborne, R.H., and Konopinska, D. (1999) Further proctolin analogues modified in the position 2 of the peptide chain and their myotropic effects in insects Tenebrio molitor and Schistocerca gregaria. Polish Journal of Pharmacology 51, 79-85.

Kuczer, M., Rosinski, G., Lisowski, M., Picur, B., and Konopinska, D. (1996) New proctolin analogues modified by D-amino acids in the peptide chain and their high cardioexcitatory effect on Tenebrio molitor. International Journal of Peptide and Protein Research 48, 286-291.

Lamango, N.S. and Isaac, R.E. (1997) Metabolism of insect neuropeptides: Properties of a membrane-bound endopeptidase from heads of Musca domestica. Insect Biochemistry And Molecular Biology 23, 801-808.

Lange, A.B. (1988) Inositol phospholipid hydrolysis may mediate the action of proctolin on insect visceral muscle. Archives Of Insect Biochemistry And Physiology 9, 201209.

Lange, A.B., Orchard, I., and Barrett, F.M. (1988) The presence and distribution of proctolin in the blood-feeding bug, rhodnius-prolixus. Journal Of Insect Physiology 34, 379-379. 
Lange, A. B., I. Orchard, et al. (1991) Evidence for the Involvement of a Schistoflrf-AmideLike Peptide in the Neural Control of Locust Oviduct. Journal of Comparative Physiology a-Sensory Neural and Behavioral Physiology 168 (3): 383-391.

Lange, A.B., Orchard, I., and Konopinska, D. (1993) The effects of selected proctolin analogues on contractions of locust (Locusta migratoria) oviducts. Journal Of Insect Physiology 39, 347-351.

Le Corronc, H. and Hue, B. (1993) Pharmacological and electrophysiological characterization of postsynaptic muscarinic receptor in the central nervous system of the cockroach. Journal of Experimental Biology 181, 257-278.

Lengvari, I., Csoknya, M., and Lubics, A. (1994) Proctolin immunoreactive element in the nerous system of earthworm (Lumbricus terrestris). Acta Biologica Hungaria 45, 337-345.

Li, C. and Calabrese, R.L. (1985) Evidence of proctolin-like substances in the central nervous system of the leech Hirudo medicinalis. Journal of Comparative Neurology 232, 414-424.

Lozano, V.C. and Gauthier, M. (1998) Effects of the muscarinic antagonists atropine and pirenzepine on olfactory conditioning in the honeybee. Pharmacology Biochemistry And Behavior 59, 903-907.

Luthman, J., Johansson, O., Ahlstrom, U., and Kvint, S. (1988) Immunohistochemical studies of the neurochemical markers, CGRP, enkephalin, galanin, gamma-MSH, NPY, PHI, proctolin, PTH, somatostatin, SP, VIP, tyrosine-hydroxylase and neurofilament in nerves and cells of the human attached gingiva. Archives Of Oral Biology 33, 149-158.

Marcel, V., Palacios, L.G., Pertuy, C., Masson, P. and Fournier, D. (1998) Two invertebrate acetylcholynesterases show activation followed by inhibition with sustrate concentration. Biochemistry 329, 329-334.

Marder, E., Abbott, L. F. et al. (1993) Physiological Insights from Cellular and Network Models of the Stomatogastric Nervous-System of Lobsters and Crabs. American Zoologist 33(1), 29-39.

Mason, C.A. (1973) New features of the brain-retrocerebral neuroendocrine complex of the locust Schistocerca vaga (Scudder). Z.Zellforch 141, 19-32. 
Maycox, P.R., Hell, J.W., and Jahn, R. (1990) Amino acid neurotransmission: spotlight on synaptic vesicles. Trends in Neuroscience 13, 83-87.

Mazzocco, C. and J. Puiroux (2000) Purification of proctolin-binding proteins from the foregut of the insect Blaberus craniifer. European Journal of Biochemistry 267(8), 2252-2259.

Mazzocco, C., Fukasawa, K.M., Auguste, P. and Jacques Puiroux (2003) Characterization of a functionally expressed dipeptidyl aminopeptidase III from Drosophila melanogaster. Eur.J.Biochem. 270, 3074 -3082.

Mobbs, P.G. (1985) Brain Structure. In: Comprehensive insect Biochemistry, Physiology and Pharmacology., 299-369. Edited by Kerkut, G.A. and Gilbert, L.I., London, Permagon Press.

Mohl, B. (1972) The control of Foregut movements by the Somatogastric Nervous system in the European House Cricket Acheta domesticus L. Journal Of Comparative Physiology 80, 1-28.

Nachman, R.J., Olender, E.H., Roberts, V.A., Holman, G.M., and Yamamoto, D. (1996) A nonpeptidal peptidomimetic agonist of the insect flrfamide myosuppressin family. Peptides 17, 313-320.

Nash, M.S., Young, K.W., JohnChalliss, R.A., Nahorski, S.R. (2001) Receptor-specific messenger oscillations. Nature 413, 381-382.

Nässel, D.R. (1993) Insect myotropic peptides: differential distribution of locustatachykinin- and leucokinin-like immunoreactive neurons in the locust brain. Cell and Tissue Research 274, 27-40.

Nässel, D.R. (1996) Neuropeptides, amines and amino acids in an elementary insect ganglion: functional and chemical anatomy of the unfused abdominal ganglion. Progress in Neurobiology 48, 325-420.

Nässel, D.R., Eckert, M., Muren, J.E., and Penzlin, H. (1998) Species-specific action and distribution of tachykinin-related peptides in the foregut of the cockroaches Leucophaea maderae and Periplaneta americana. Journal of Experimental Biology 201, 1615-1626.

Nässel, D.R., Holmqvist, B.I., and Moverus, B.J.A. (1989) Vasopressin- and proctolin-like immunoreactive efferent neurons in blowfly abdominal ganglia: Development and ultrastructure. Journal of Comparative Neurology 283, 450-463. 
Nässel, D.R. and O'Shea, M. (1987) Proctolin-like immunoreactive neurons in the blowfly central nervous system. Journal of Comparative Neurology 265, 437-454.

Nässel, D.R., Passier, P.C.C.M., Elekes, K., Dircksen, H., Vullings, H.G.B., and Cantera, R. (1995) Evidence that locustatachykinin I is involved in release of adipokinetic hormone from the locust corpora cardiaca. Regulatory Peptides 57, 297-310.

Nässel, D.R. (1993) Neuropeptides in the insect brain: a review. Cell and Tissue Research 273, 1-29.

Nishizuka, Y. (1992) Intracellular signaling by hydrolysis of phospholipids and activation of protein kinase C. Science 258, 607-614.

Nusbaum, M.P., Blitz, D.M., Swensen, A.M., Wood, D. and Marder, E. (2001) The roles of co-transmission in neural network modulation. Trends Neuroscience 24(3), 146-54.

Ocker, W.G., Hedwig B. and Elsner, N. (1995) Application of putative neurotransmitters elicits and modulates stridulation in two species of grasshoppers. Journal of Experimental Biology 198, 1701-1710.

O'Shea, M. and Adams, M.E. (1981) Pentapeptide (proctolin) associated with an identified neuron. Science $213,567-569$.

O'Shea, M. and Adams, M.E. (1981) Pentapeptide (proctolin) associated with an identified neuron. Science 213, 567-569.

O'Shea, M. and Bishop, C.A. (1982) Neuropeptide proctolin associated with an identified skeletal motoneuron. Journal of Neuroscience 2, 1242-1251.

Orchard, I., Belanger, J.H., and Lange, A.B. (1989) Proctolin - a review with emphasis on insects. Journal of Neurobiology 20, 470-496.

Osborne, R.H., Banner, S.E., and Wood, S.J. (1990) The Pharmacology of the gut of the Desert Locust Shistocerca gregaria and other Insects. Comparative Biochemistry And Physiology C 96C, 1-9.

Osborne, R.H., Bradford, H.F., and Jones, D.G. (1973) Patterns of amino acid release from nerve-endings isolated from spinal cord and medulla. Journal Of Neurochemistry 21, 407-419.

Osborne, R.H., Odell, B., and Blagbrough, I.S. (1995) Proctolin and related N-methylated pentapeptides selectively contract locust foregut but not rat ileum. Bioorganic and Medicinal Chemistry Letters 5, 2085-2088. 
Pasztor, V.M. and Bush, B.M.H. (1989) Peripheral modulation of mechanosensitivity in primary afferent neurons. Nature 326, 793-795.

Pasztor, V.M. and MacMillan, D.L. (1988) Diverse modulatory effect of proctolin, octopamune, FRMH amide and serotonin on crayfish mechanoreceptors. Soc. Neurosci. Abstr, 14, 377

Pasztor, V.M. and Golas, L.B. (1993) The modulatory effects of serotonin, neuropeptide $\mathrm{F} 1$ and proctolin on the receptor muscles of the lobster abdominal stretch receptor and their exoskeletal muscle homologues. Journal of experimental Biology 174, 363-374.

Penzlin, H. (1985) Stomatogastric nervous system. In: Comprehensive insect Biochemistry, Physiology and Pharmacology., 371-406. Edited by Kerkut, G.A. and Gilbert, L.I., London, Permagon Press.

Penzlin, H., Eckert, M., and Kusch, T. (1980) Distribution of proctolin in the sixth abdominal ganglion of Periplaneta americana, and the effect of proctolin on the ileum of mammals. In: Advances in Physiology, 22 Ed., 525-535. Edited by Rosza, K.S., Oxford, Permagon Press.

Pflüger, H.J. and Watson, A.D.H. (1988) Sturcture and distribution of dorsal unpaired median (DUM) neurons in the abdominal nerve cord of male and female locusts. The Journal of Comparative Neurology 268, 329-345.

Pickering, B.T. (1978) Essays in Biochemistry, 45-81, London, Academic Press.

Piek, T. (1997) Solitary wasp venoms and toxins as tools for the study of neuro- muscular transmission in insects. Ciba Foundation Symposia 1982, 275-290.

Piek, T., Visser, B.J., and Mantel, P. (1978) Effect of proctolin. BPP5a and related peptides on rhythmic contractions in Locusta migratoria. Comparative Biochemistry And Physiology C 62C, 151-154.

Plotnikova, S.I. (1967) The innervation of the gut in Locusta migratoria, (Othoptera, Acrididae). Entomological Review 46, 69-71.

Poulet, J.F.A and Hedwig, B. (2001) Tympanic membrane oscillations and auditory receptor activity in the stridulating cricket Grillus bimaculatos. The Journal of experimental Biology 204, 1281-1293.

Puiroux, J. and Loughton, B.G. (1992) Degradation of the neuropeptide proctolin by membrane-bound proteases of the hindgut and ovary of locusta-migratoria and the 
effects of different inhibitors. Archives Of Insect Biochemistry And Physiology 19, 193-202.

Puiroux, J., Pedelaborde, A., and Loughton, B.G. (1993) The effect of proctolin analogues and other peptides on locust oviduct muscle contractions. Peptides 14, 11031109.

Rhee, S.G. and Choi, K.D. (1992) Regulation of inositol phospholipid-specific phospholipase C isozymes. Journal of Biological Chemistry 267, 12393-12396.

Roberts, F., Hill, R.G., Osborne, R.H., and Mitchell, J.F. (1979) The effect of depolorising potassium concentrations on the efflux of GABA from rat dorsal medulla in vivo and from slices and synaptosomes. Brain Research 178 , 467-477.

Saver, M. A., Wilkens, J.L. and Airriess, C.N. (1998) Proctolin affects the activity of the cardiac ganglion, myocardium, and cardioarterial valves in Carcinus maenas hearts. Journal of comparative Physiology B 168, 473-482.

Schoofs, L., W. Schroven, et al. (1990) The Occurrence of Myotropic Factors in the Intestine and Malpighian Tubules of Locusta-Migratoria. Comparative Biochemistry and Physiology C-Pharmacology Toxicology \& Endocrinology 97 (1), 195-199

Schoofs, L. and Holman, G.M. (1995) Regulatory peptides in insects - preface. Regulatory Peptides 57, R 3-R 4

Schoofs, L., Tips, A., Holman, G.M., Nachman, R.J., and Deloof, A. (1992) Distribution of locustamyotropin-like immunoreactivity in the nervous-system of Locusta migratoria. Regulatory Peptides 37, 237-254.

Schoofs, L., Veelaert, D., Vanden Broeck, J., and De Loof, A. (1996) Immunocytochemical distribution of Locustamyoinhibiting peptide (Lom-MIP) in the nervous system of Locusta migratoria. Regulatory Peptides 63, 171-179.

Schoofs, L., Veelaert, D., Vanden Broeck, J., and De Loof, A. (1997) Peptides in the locusts, Locusta migratoria and Schistocerca gregaria. Peptides 18, 145-156.

Schooneveld, H., Tesser, G.I., Veenstra, J.A., and Romberg Privee, H.M. (1983) Adipokinetic hormone and $\mathrm{AKH}$-like peptide demonstrated in the corpora cardiaca and nervous system of Locusta migratoria by immunocytochemistry. Cell and Tissue Research 230, 67-76.

Schultz, G. and Penzlin, H. (1991) Proctolin - Effects on brain and muscle in rats. Kemiai Kozlemenye 58, 432-438. 
Schultz, H., Schwartzberg, H., and Penzlin, H. (1981) The insect neuropeptide proctolin can affect the CNS and the smooth-muscle of mammals. Acta Biologica Medica Germany 40, K1-K5

Schurmann, F. W., R. Sandeman, et al. (1991) Dense-Core Vesicles and Nonsynaptic Exocytosis in the Central Body of the Crayfish Brain. Cell and Tissue Research 265 (3), 493-501.

Schütze H, Elsner N (2001) Stridulatory pattern generation in acridid grasshoppers: metathoracic interneurons in Stenobothrus rubicundus (Germar 1817). Journal of Comparative Physiology A 187, 529-540.

Schwarz, T.L., LEE, G.M.H., Siwicki, K.K., and Standaert, D.G. (1984) Proctolin in the lobster - the distribution, release, and chemical characterisation of a likely neurohormone. Journal of Neuroscience 4, 1300-1311.

Selverston, A.I. (1980) Are central pattern generators understandable? Behaviour Brain Science 3, 535-571.

Sharp, A.A., O'Neil, M.B., Abbott, L.F. and Marder, E. (1993) Dynamic clamp: computergenerated conductances in real neurons. Journal of Neurophysiology 69 (3), $992-$ 995.

Skiebe, P. and O. Ganeshina (2000) Synaptic neuropil in nerves of the crustacean stomatogastric nervous system: An immunocytochemical and electron microscopical study. Journal of Comparative Neurology 420 (3), 373-397.

Skiebe, P. (2001) Neuropeptides are ubiquitouse chemical mediators: using the stomatogastric nervous system as a model system. The Journal of Experimental Biology 204, 2035-2048.

Sladeczek, F., Pin, J.P., Recasens, M., Bockaert, J. and Weiss, S. (1985) Glutamate stimulates inositol phosphate formation in striatal neurones. Nature 317, 717-719.

Snodgrass, R.E. (1935) Insect Morphology, McGraw Hill.

Sombati, S. and Hoyle, G. (1984) Generation of specific behaviours in a locust by local release into neuropil of the natural neuromodulator octopamine. J. Neurobiol. 15, 481-506.

Spittaels, K., Vankeerberghen, A., Torrekens, S., Devreese, B., Grauwels, L., Van Leuven, F., Hunt, D., Shabanowitz, J., Schoofs, L., Van Beeumen, J., and De Loof, A. (1995) Isolation of Ala1-proctolin, the first natural analogue of proctolin, from the 
brain of the Colorado potato beetle. Molecular and Cellular Endocrinology 110, 119-124.

Starratt, A.N. and Brown, B.E. (1975) Structure of the pentapeptide proctolin, a proposed neurotransmitter in insects. Life Sciences 17, 1253-1256.

Starratt, A.N. and Brown, B.E. (1979) Analogues of the insect myotropic peptide proctolin: synthesis and structure-activity studies. Biochemical And Biophysical Research Communications 90, 1125-1130.

Starratt, A.N., Orchard, I., Lange, A.B., and Steele, R.W. (1997) Comparison of the myotropic activity of position-2 modified analogues of proctolin on the hindgut of Periplaneta americana and the oviduct of Locusta migratoria. Journal Of Insect Physiology 43, 931-938.

Starratt, A.N. and Steele, R.W. (1984) In vivo inactivation of the insect neuropeptide proctolin in Periplaneta-americana. Insect Biochemistry 14, 97-102.

Steele, R.W. and Starratt, A.N. (1985) In vitro inactivation of the insect neuropeptide Proctolin in Haemolymph from Periplaneta americana. Insect Biochemistry 15 , 511-519.

Storm-Mathieson, J. (1977) Localisation of transmitter candidates in the brain: the hippocampal formation as a model. Progress in Neurobiology 8, 119-181.

Sullivan, R.E. (1979) A proctolin-like peptide in crab pericardial organs. Journal of experimental Zoology 210, 543-552.

Swales, L.S. and Evans, P.D. (1988) Histochemical localization of octopamine- and proctolin- sensitive adenylate cyclase activity in a locust skeletal muscle. Histochemistry 90, 233-239.

Swensen, A.M. and Marder, E. (2000) Multiple peptides converge to activate the same voltage-dependent current in a central pattern-generating circuit. The Journal of Neuroscience 20(18), 6752-6759.

Sylvia, V.L. and Holman, G.M. (1983) Isolation and high-performance liquidchromatographic purification of a myotropic peptide from the hindgut of the crayfish, Procambarus clarkii. Journal Of Chromatography 261, 158-162.

Thompson, K.J. and Roosevelt, J.L. (1998) Comparason of neural elements in sexually dimorphic segments of the grasshopper, Schistocerca gregaria. The Journal of Comparative Neurology 394, 14-28. 
Trimmer, B.A. and Weeks, J.C. (1993) Muscarinic Acetylcholine Receptors Modulate the Excitability of an Identified Insect Motoneuron. Journal of neurophysiology 69, $1821-1836$

Tublitz, N.J. and Truman, J.W. (1985) Insect cardioactive peptides. 1. Distribution and molecular characteristics of two cardioacceleratory peptides in the tobacco hawkmoth, Manduca sexta. Journal of Experimental Biology 114, 365-379.

Turner, A., Matsas, R., and Kenny, J. (1985) Are there neuropeptide-specific peptidases? Biochemical Pharmacology 34, 1347-1356.

Turrigiano, G.G., Marder, E. (1993) Modulation of identified stomatogastric ganglion neurons in primary cell culture, Journal of Neuropyisiology. 69, 1993-2002.

Ude, J. and Eckert, M. (1988) Submicroscopic characterisation of proctolin-like immunoreactivity in the nervous-system of the cockroach Periplaneta americana $L$. Cell and Tissue Research 254, 197-202.

Van den Beukel. I., Regina, G.D.M. van Keef, Zwart, R., Oortgiesen, M. (1998) Physiostigmine and acetylcholine differentially activate nicotinic receptor subpopulations in Locusta migratoria neurons. Brain Research 789, 263-273.

Van Staaden, M.J. and Huber, R. (2001) Multidisciplinary dissection of behavioral arousal: The role of muscarinic acetylcholine stimulation in grasshopper stridulatory behaviour. Proc Nat Acad Sci USA 98, 9468-9470.

Veelaert, D., Schoofs, L., and De Loof, A. (1998) Peptidergic control of the corpus cardiacum - corpora allata complex of locusts. International Review Of Cytology 182, 249-302.

Veelaert, D., Schoofs, L., Tobe, S.S., Yu, C.G., Vullings, H.G.B., Couillaud, F., and De Loof, A. (1995) Immunological evidence for an allatostatin-like neuropeptide in the central nervous system of Schistocerca gregaria, Locusta migratoria, and Neobellieria bullata. Cell and Tissue Research 279, 601-611.

Veenstra, J.A., RombergPrivee, H.M., and Schooneveld, H. (1985) A proctolin-like peptide and its immunocytochemical localization in the Colorado potato beetle, Leptinotarsa decemlineata. Cell and Tissue Research 240, 535-540.

Walther, C., Zitlau, K.E., Murck, H. and Voigt, K. (1998) Resting Membrane Properties of Locust Muscle and Their Modulation I. Actions of the Neuropeptides YGGFMRFamide and Proctolin. Journal of Neurophysiology 80, 771-784. 
Wang, Z. and Orchard, I. (1995) Ultrastructural and immunocytochemical studies of neuromuscular junctions in oviduct of Locusta migratoria. Cell and Tissue Research 279, 591-599.

Washio, H. and T. Koga. (1990) Proctolin and Octopamine Actions on the Contractile Systems of Insect Leg Muscles. Comparative Biochemistry and Physiology CPharmacology Toxicology \& Endocrinology 97 (2), 227-232.

Watson $3^{\text {rd }}$, W.H. and Hoshi, T. (1985) Proctolin induces rhythmic contractions and spikes in Limulus heart muscle. Am J Physiol Regul Integr Comp Physiol 249, R490R495.

Weevers, R.d. (1985) The insect ganglia. In: Comprehensive insect Biochemistry, Physiology and Pharmacology., 213-297. Edited by Kerkut, G.A. and Gilbert, L.I., London, Permagon Press.

Wegener, C., Nässel, D. R. (2000) Peptide-Induced Ca ${ }^{2+}$ Movements in a Tonic Insect Muscle: Effects of Proctolin and Periviscerokinin-2. Journal of Neurophysiology 84, 3056-3066.

Wenzel, B., Elsner, N. and Hedwig, B. (1998) Microinjection of neuroactive substances into brain neuropil controls stridulation in the cricket Gryllus bimaculatus (de Geer). Naturwissenschaften 85, 452-454.

Wenzel, B. and Hedwig, B. (1999) Neurochemical control of cricket stridulation revealed by pharmacological microinjections into the brain. The Journal of Experimental Biology 202, 2203-2216.

Wenzel, B., Elsner, N. and Heinrich R. (2001) mAChRs in the Grasshopper Brain Mediate Excitation by Activation of the AC/PKA and the PLC Second-Messenger Pathways. J Neurophysiol 87: 876-888.

Werman, R. (1966) Criteria for identification of a central nervous system transmitter. Comparative Biochemistry And Physiology 18, 745-766.

Wigglesworth, V.B. (1972) The principles of Insect Physiology, 7 Ed., London, Chapman and Hall.

Williams, J.L.D. (1975) Anatomical studies of the insect central nervous system: A groundplan of the midbrain and an introduction to the central complex in the locust, Schistocerca gregaria (Orthoptera). Journal of Zoology 176, 67-86. 
Wood, D.E., Nishikawa, M., and Derby, C.D. (1996) Proctolinlike immunoreactivity and identified neurosecretory cells as putative substrates for modulation of courtship display behavior in the blue crab, Callinectes sapidus. Journal of Comparative Neurology 368, 153-163.

Wood, S.J., Osborne, R.H., Banner, S.E., and Cattell, K.J. (1992) Effects of FMRFamiderelated peptides and morphine on the isolated foregut of the locust Schistocerca gregaria. Comparative Biochemistry And Physiology C 103, 315-320.

Wood, S.J., Osborne, R.H., Gomez, S., and Jewess, P.J. (1992) Pharmacology of the isolated foregut of the Locust Shistocerca gregaria - IV. Characterisation of a Muscarinic Acetylcholine receptor. Comparative Biochemistry And Physiology C 103C, 527-534.

Wood, S. J. and R. H. Osborne (1991) Is Sulfotep a Proctolin Receptor Antagonist. Pesticide Science 32 (4), 485-491.

Wood, S.J., Osborne, R.H., Cattell, K.J., and Banner, S.E. (1990) FMRFamide and related peptides modulate the actions of 5- hydroxytryptamine and proctolin on the foregut of the locust, Schistocerca gregaria. Biochemical Society Transactions 18, 384385.

Woynica, I., Rosinski, G. and Konopinska, D. (2002) New Proctolin Analogues Modified in Position 2 or 3 of the Peptide Chain and Their Myotropic Effects in Insects. Polish Journal of Chemistry, 76, 1425-1431.

Yamada, M., Inanobe, A. and Kurachi, Y.A. (1998) G Protein Regulation of Potassium Ion Channels. Pharmacological Reviews 50 (4), 723-757.

Yasuyama, K., T. Kimura, et al. (1992) Proctolin-Like Immunoreactivity in the Dorsal Unpaired Median Neurons Innervating the Accessory-Gland of the Male Cricket, Gryllus-Bimaculatus. Zoological Science 9 (1), 53-64.

Yazawa, T. and K. Kuwasawa (1992) Intrinsic and Extrinsic Neural and Neurohumoral Control of the Decapod Heart. Experientia 48 (9), 834-840.

Zheng, J., Leong, D., Lees, G., and Nicholson, R.A. (1998) Studies on the interaction of surangin B with insect mitochondria, insect synaptosomes, and rat cortical neurons in primary culture. Pesticide Biochemistry And Physiology 61, 1-13. 


\section{Appendix}

Grasshopper saline solution (Clements and May, 1974):

$140 \mathrm{mM} \quad \mathrm{NaCl}$

$5 \mathrm{mM} \quad \mathrm{KCl}$

$4 \mathrm{mM} \quad \mathrm{NaH}_{2} \mathrm{PO}_{4} \cdot 2 \mathrm{H}_{2} \mathrm{O}$

$5 \mathrm{mM} \quad \mathrm{Na}_{2} \mathrm{HPO}_{4}$

$2 \mathrm{mM} \quad \mathrm{CaCl}_{2} \cdot 2 \mathrm{H}_{2} \mathrm{O}$

$\mathrm{pH}=7,2$ 


\section{Lebenslauf}

Stoyan Raykov Vezenkov

Nationalität: bulgarisch

17.09.1974 Geboren als zweites Kind von Svetla Nikiforova Vezenkova und Rayko Stoyanov Vezenkov in Blagoevgrad (Bulgarien)

1988-1992 Besuch des Sergei Pavlovitch Koroljov Gymnasiums in Blagoevgrad

Juni 1992 Diplom für Oberschulabschluss

1992-1997 Studium der Molekular Biologie an der St. Kliment Ohridski Universität in Sofia

Sept. 1997 Studiumsabschluss (Magister der Molekular Biologie)

1998-1999 Ableistung des Wehrdienstes

18.08.1999 Geburt des ersten Kindes Veronika Stoyanova Vezenkova

1999-2001 Biologielehrer am Sergei Pavlovitch Koroljov Gymnasium in Blagoevgrad

März 2001 Wissenschaftlicher Assistent an der Söderntörn Högskola Universität in -Juni 2001 Stockholm (Schweden)

Juni 2001 Beginn der Promotion im Labor von Prof. Dr. N. Elsner am Institut für Zoologie, Anthropologie und Entwicklungsbiologie, Abteilung Neurobiologie, der Georg-August-Universität Göttingen 